\title{
Viscoelasticity, Like Forces, Plays a Role in Mechanotransduction
}

\author{
Claudia Tanja Mierke* \\ Faculty of Physics and Earth Science, Peter Debye Institute of Soft Matter Physics, Biological Physics Division, University of \\ Leipzig, Leipzig, Germany
}

\section{OPEN ACCESS}

Edited by:

Fabian Spill,

University of Birmingham,

United Kingdom

Reviewed by:

Jose Manuel Garcia-Aznar,

University of Zaragoza, Spain

Cynthia Hajal,

Dana-Farber Cancer Institute,

United States

*Correspondence:

Claudia Tanja Mierke

claudia.mierke@uni-leipzig.de

Specialty section:

This article was submitted to

Cell Adhesion and Migration,

a section of the journal

Frontiers in Cell and Developmental

Biology

Received: 05 October 2021

Accepted: 11 January 2022

Published: 09 February 2022

Citation:

Mierke CT (2022) Viscoelasticity, Like

Forces, Plays a Role

in Mechanotransduction.

Front. Cell Dev. Biol. 10:789841.

doi: 10.3389/fcell.2022.789841
Viscoelasticity and its alteration in time and space has turned out to act as a key element in fundamental biological processes in living systems, such as morphogenesis and motility. Based on experimental and theoretical findings it can be proposed that viscoelasticity of cells, spheroids and tissues seems to be a collective characteristic that demands macromolecular, intracellular component and intercellular interactions. A major challenge is to couple the alterations in the macroscopic structural or material characteristics of cells, spheroids and tissues, such as cell and tissue phase transitions, to the microscopic interferences of their elements. Therefore, the biophysical technologies need to be improved, advanced and connected to classical biological assays. In this review, the viscoelastic nature of cytoskeletal, extracellular and cellular networks is presented and discussed. Viscoelasticity is conceptualized as a major contributor to cell migration and invasion and it is discussed whether it can serve as a biomarker for the cells' migratory capacity in several biological contexts. It can be hypothesized that the statistical mechanics of intra- and extracellular networks may be applied in the future as a powerful tool to explore quantitatively the biomechanical foundation of viscoelasticity over a broad range of time and length scales. Finally, the importance of the cellular viscoelasticity is illustrated in identifying and characterizing multiple disorders, such as cancer, tissue injuries, acute or chronic inflammations or fibrotic diseases.

Keywords: cell mechanics, extracellular matrix constraints, organelles, viscoelasticity, cancer cells, stiffness, biophysical techniques, hallmarks of migration and invasion

\section{INTRODUCTION TO THE PHENOMENON OF VISCOELASTICITY IN CELLS}

The phenomenon of viscoelasticity can be found in nature in several kinds of material, such as in the most prominent example, rubber, and can be employed in engineering of synthetic or biological materials. A material behaves elastically according to Hooke's law if the applied stress is proportional to the strain generated in the material, which is true up to a certain level of stress. No material behaves exclusively in a purely elastic manner, but many of these materials can be modeled as one elastic material, which is especially the case when the strain is small. A key assumption in an exclusive elastic material is that the energy required for deformation is stored in the material and is released with its entire efficiency when the material returns to its initial state after deformation. Viscoelasticity is by definition a state of thickness or toughness based on the internal structure of the material. In viscous materials, which include liquids, this means that the energy required to deform it, is partly converted as heat, which is related to internal structural losses. In viscous materials, the force is proportional to 
the amount of change in deformation. It is generally accepted that some materials can be assumed to be purely elastic or purely viscous. Viscoelasticity can therefore be defined as a property of a material that has both an elastic and a viscous component.

In biology, viscoelasticity refers to the property of living matter, including cells, spheroids, and tissues, that manifest both viscous and elastic properties when subjected to deformation. In the past, it was often assumed that cells behave in a purely elastic way, but this often turned out to be a too simplistic view of things. However, although cells are viscoelastic, it is not always straightforward to evaluate or simulate viscoelasticity, and adopting a simplified elastic behavior of cells for this purpose may be a reasonable alternative. The viscoelastic or material characteristics of cells, spheroids and tissues act as major regulators of cell and tissue growth, cell motility, and tissue homeostasis (Barriga and Mayor, 2019; Burla et al., 2019; Petridou and Heisenberg, 2019; Chaudhuri et al., 2020). Biological systems, including active matter, exhibit viscoelasticity, which allows them to maintain a fundamental architectural structure owing to their solid-like nature, but at the selfsame time to dynamically rearrange themselves into various conformations and modes owing to their viscous nature (Lecuit et al., 2011; Clément et al., 2017; Pegoraro et al., 2017; Petridou and Heisenberg, 2019). At the cellular length scale, viscoelasticity affects various single-cell features such as conformation, division, and movement, and is primarily governed by the physical characteristics of the supporting cytoskeletal systems (Pegoraro et al., 2017). Viscoelasticity at the tissue length scale has been shown to play an integral role in collective morphogenetic events such as tissue involution, spreading, injury repair, and migratory processes, and is primarily governed by the concert of cell-cell and/or cell-extracellular space interfaces (Petridou et al., 2017; Barriga and Mayor, 2019; Chaudhuri et al., 2020).

Cell, spheroids and tissues possess a multitude of inelastic characteristics, among them are viscoelasticity, plasticity and fracture. When mechanically deformed, it is usually impossible to avoid unfolding protein domains, unbinding cytoskeletal crosslinkers, dividing organelle assemblies, breaking weak sacrificial bonds, disrupting transient cell-matrix and cell-cell adhesions and inducing gene expression through translocation of transcription factors into the nucleus. All of which are regarded as inelastic. These structural transformations are mostly reversible and consequently not plastic in the strict meaning of the word, however they dissipate significant quantities of elastic energy through structural attenuation (Gralka and Kroy, 2015). The inelastic reaction can be observed as gradual or partial recovery of the material after elimination of the forces that caused the deformation (Gavara et al., 2008). Besides, it is rational that the deformation is considered to be a function of the history of the exerted forces. Viscoelastic materials display three prominent characteristics: stress relaxation, which is the stress decreases with time (a response of a viscoelastic material to a constant strain step), creep (a constant stress with a decrease in strain with time), and hysteresis (an incongruence between loading and unloading processes) (Banks et al., 2011). The term "viscoelasticity" combines both types of mechanical response, the response of elastic solids and viscous fluids. Consequently, not merely solids, but also liquids are susceptible of displaying such a property. Yet the manner in which they react to a mechanical cue differs widely. The response of a fluid to a given deformation under any two conditions would be identical, whereas a solid, for example, would respond differently in its original form and after a deformation. More generally, for solids, pure strains can affect the reaction of the material, while rotations can have no effect (Truesdell et al., 2004). When addressing viscoelasticity, it has to be taken into account that the inelastic response is also present (Trepat et al., 2007), even though the response may not really be permanent or irreversible and can also be reversible plastic (Gralka and Kroy, 2015). Moreover, viscoelastic characteristics of cells have been proposed to play a key role in the regulation of cellular functions, such as motility (Barriga and Mayor, 2019; Burla et al., 2019; Petridou and Heisenberg, 2019; Chaudhuri et al., 2020). Viscoelastic characteristics of cells are proposed to act as critical new biomarkers of disease status and advancement (Bao and Suresh, 2003). The most straightforward attempt to specify the viscoelastic characteristics of cells focuses on two variables: Stiffness and viscosity, which typify the elastic and dissipative elements of a cell's reaction to stress (Moeendarbary and Harris, 2014). Elastic reaction has been widely adopted as a biomarker for cancer cells (Cross et al., 2007) or metastatic capacity (Xu et al., 2012). In addition, elasticity has been implicated in cell migration patterns occurring in embryogenesis (Barriga et al., 2018). Cell viscosity has been associated with numerous biological events, including diffusion (Wojcieszyn et al., 1981), porous traffic and deformability of erythrocytes (Lim et al., 2006) and the state of cellular disease (Eze, 1992; Zakim et al., 1992). Therefore, it can be hypothesized that the viscoelastic properties of cells may serve as a novel biomarker for cellular motility and thus for the progression of diseases such as cancer metastasis, in which cell invasiveness plays a key role.

Following the introduction and explanation of the phenomenon of viscoelasticity, the review consists of presenting and discussing biophysical techniques for determining viscoelasticity. The review pursues a hierarchical structure that reflects the spatial scale, ranging from firstly molecular mechanosensing between cells and cell matrix, to secondly transcriptional regulation leading to thirdly changes in cell state, such as epithelial to mesenchymal transition (EMT), encompassing single cell migration, to fourthly multicellular processes, including collective migration, spheroid and tumeroid biology, and fifthly disease states such as cancer, fibrosis, and others.

\section{BIOPHYSICAL TECHNIQUES FOR ANALYZING VISCOELASTICITY \\ 2.1 Classical Biophysical Techniques to Analyze Viscoelasticity}

The majority of approaches to query the viscoelastic behavior of cells utilize forced induced deformations or probes (Wirtz, 2009) on relatively short time scales due to experimental requirement 


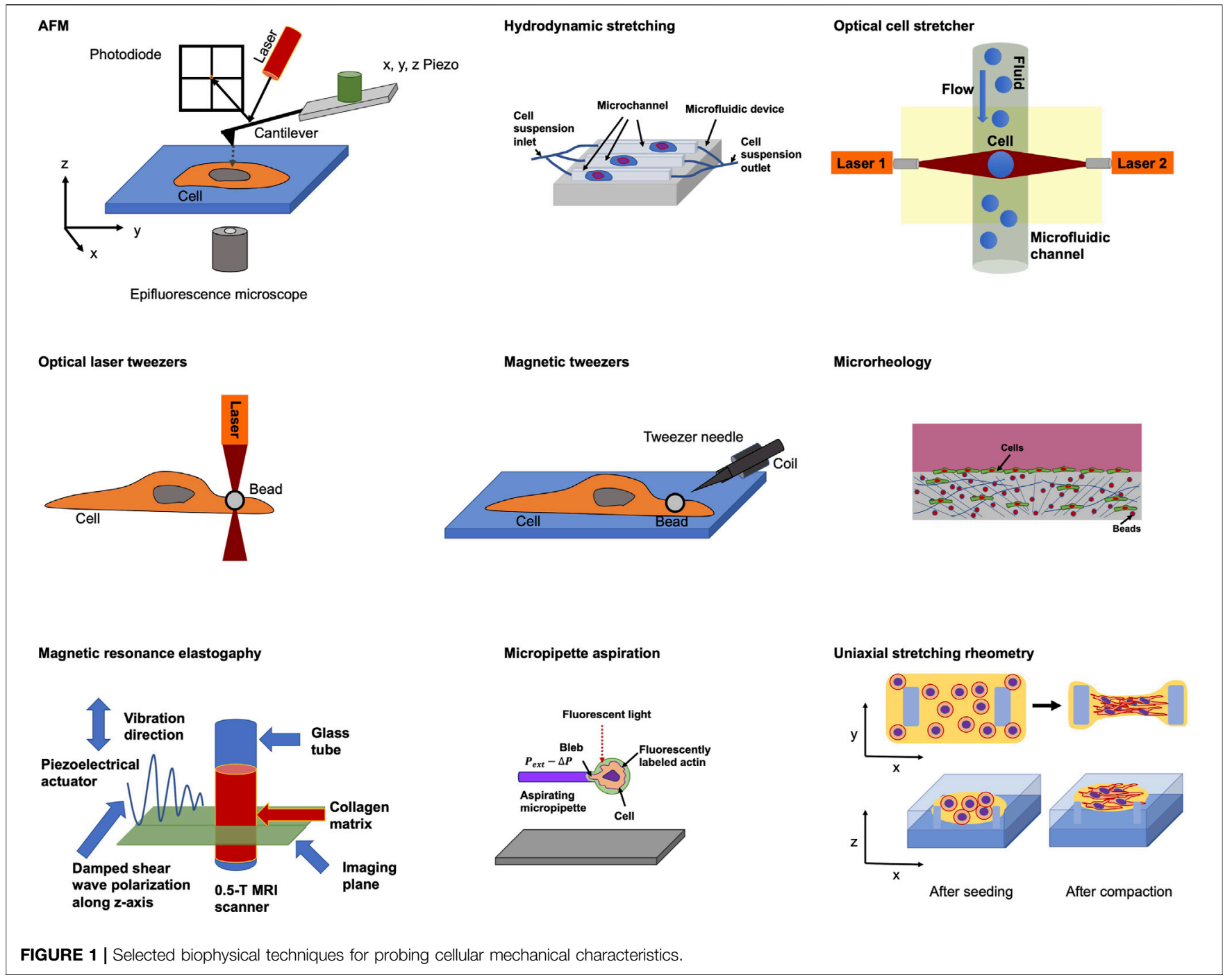

and feasibility. Efforts to gauge the elastic aspect of cell viscoelasticity involve atomic force microscopy (AFM) (Haase and Pelling, 2015; Fischer et al., 2017, Mierke et al., 2017, Fischer et al., 2020), hydrodynamic stretching (Gossett et al., 2012), optical cell stretcher (Kunschmann et al., 2017, Kunschmann et al., 2019; Mierke et al., 2020), optical laser tweezers (Lincoln et al., 2004), magnetic tweezers (Amblard et al., 1996; Aermes et al., 2020, Aermes et al., 2021), microrheology (Mason and Weitz, 1995; Crocker and Hoffman, 2007), magnetic resonance elastography (Muthupillai et al., 1995; Zhang et al., 2021), micropipette suction (Hochmuth, 2000) and uniaxial stretching rheometry (Desprat et al., 2005) (Figure 1). The viscous part of the reaction of cells to mechanical probing has been determined employing biophysical techniques encompassing microrheology (Mason and Weitz, 1995; Crocker and Hoffman, 2007), micropipette suction (Hochmuth, 2000), fluorescent rotor protein (Kuimova et al., 2008), AFM (Rebelo et al., 2013), electronic spin resonance (Mastro and Keith, 1984) and optical laser tweezers (Ługowski et al., 2002) (Table 1).
An overview of these selected biophysical techniques and their characteristics are provided in (Table 1), which also lists major advantages and disadvantages. The Table $\mathbf{1}$ also summarizes whether a specific technique has been applied to a 3D model, such as spheroids and organoids, or tissue specimen. Most of the biophysical techniques, including QPI, AFM, Microrheology, Fluorescent rotor protein analysis, Electronic spin resonance and MRE have been adapted to measure additionally 3D models, or tissues. In the following, selected prominent major biophysical techniques are briefly introduced and their potential for probing viscoelasticity is highlighted. Moreover, it is pointed out whether a biophysical technique is suitable for employment in a 1D, 2D, 3D model system and/or tissue specimen (Table 1). Specifically, Optical cell stretcher, Magnetic tweezers, Micropipette suction and Uniaxial stretching rheometry can solely been used in 2D model systems (Table 1) and are most often utilized to determine the mechanical characteristics of single cells, including viscoelasticity.

The measurement of the viscoelastic characteristics of active matter, such as cells, spheroids and tissues, has expanded in the 
TABLE 1 | Most important characteristics of specific biophysical techniques.

\begin{tabular}{|c|c|c|c|c|c|}
\hline $\begin{array}{l}\text { Biophysical } \\
\text { technique }\end{array}$ & Characteristics & Advantages & Disadvantages & $\begin{array}{l}\text { Test } \\
\text { material }\end{array}$ & $\begin{array}{l}\text { 1D, } \\
\text { 2D } \\
\text { or } \\
3 D\end{array}$ \\
\hline \multirow{6}{*}{$\begin{array}{l}\text { Quantitative phase } \\
\text { imaging (QPI) }\end{array}$} & Tracks & Probe- and contact-free & Excitation/emission are time limited & SC & $2 \mathrm{D}$ \\
\hline & $\begin{array}{l}\text { Displacement of cells due to shear flow to } \\
\text { assess cell stiffness }\end{array}$ & Fluctuations & $\begin{array}{l}\text { (photobleaching), can reduce cell viability } \\
\text { and stress the cell physiology (photoxicity) }\end{array}$ & $\mathrm{CC}$ & $3 \mathrm{D}$ \\
\hline & Measurement of biomass movement & Unlabeled specimens & & $\mathrm{SP}$ & \\
\hline & Viscoelasticity & Low phototoxicity & & OR & \\
\hline & $\begin{array}{l}\text { Contrast agents are inserted into the cell } \\
\text { (dyes, nanoparticles) or induced via } \\
\text { genetic mutation }\end{array}$ & No photobleaching & & TS & \\
\hline & Cell membrane fluctuations & $\begin{array}{l}\text { Objective measure of morphology } \\
\text { and dynamics, free of variability } \\
\text { due to contrast agents }\end{array}$ & & & \\
\hline \multirow{2}{*}{$\begin{array}{l}\text { Quantitative phase } \\
\text { rheology (QPR) }\end{array}$} & Viscoelasticity & Probe- and contact-free & Flat substrate & SC & $2 \mathrm{D}$ \\
\hline & Shear stiffness measurement & $\begin{array}{l}\text { Single-shot imaging of cellular } \\
\text { rheologic properties } \\
\text { Fluctuations }\end{array}$ & & $\mathrm{CC}$ & $3 \mathrm{D}$ \\
\hline \multirow[t]{5}{*}{$\begin{array}{l}\text { Atomic force } \\
\text { microscopy (AFM) }\end{array}$} & $\begin{array}{l}\text { Indents cellular surface with a micron- } \\
\text { sized bead attached to a cantilever while } \\
\text { the motion of the cantilever is monitored } \\
\text { by an optical sensor }\end{array}$ & $\begin{array}{l}\text { Probing of specific receptors of } \\
\text { cells with coated cantilevers }\end{array}$ & Low throughput & SC & $2 \mathrm{D}$ \\
\hline & $\begin{array}{l}\text { Measurement of molecules, cell } \\
\text { organoids and cells to whole tissue }\end{array}$ & $\begin{array}{l}\text { Adherent and suspended cell } \\
\text { measurements }\end{array}$ & Cells needs to be on the surface & $\mathrm{CC}$ & $3 \mathrm{D}$ \\
\hline & Elasticity & Relatively low throughput & & $\mathrm{SP}$ & \\
\hline & Viscoelasticity & 1 cell per min & & OR & \\
\hline & & & & TS & \\
\hline \multirow[t]{4}{*}{$\begin{array}{l}\text { Hydrodynamic } \\
\text { stretching }\end{array}$} & $\begin{array}{l}\text { Microfluidic chip in which optical fibers } \\
\text { have been arranged in a post-processing } \\
\text { step }\end{array}$ & High throughput & Without 3D matrix environment & SC & $1 \mathrm{D}$ \\
\hline & $\begin{array}{l}\text { Microscopically observed cell transit } \\
\text { through microchannels or pores }\end{array}$ & $\begin{array}{l}\text { Capable of probing single-cell } \\
\text { deformability at approximately } \\
2,000 \text { cells/s }\end{array}$ & Flow or liquid effect & & \\
\hline & Elasticity & Label-free & $\begin{array}{l}\text { Cell size and adhesiveness can contribute } \\
\text { to the measurement }\end{array}$ & & \\
\hline & Viscoelasticity & $\begin{array}{l}\text { Computationally analyzed to } \\
\text { extract quantitative parameters }\end{array}$ & & & \\
\hline
\end{tabular}

\begin{tabular}{|c|c|c|c|c|c|}
\hline \multirow[t]{4}{*}{ Optical cell stretcher } & Optically deformation of the whole cell & Relatively high cell numbers & $\begin{array}{l}\text { Single cells or small cell cluster without 3D } \\
\text { matrix environment }\end{array}$ & SC & $2 \mathrm{D}$ \\
\hline & Viscoelasticity & 1 cell per min & $\begin{array}{l}\text { Rounded cells without protrusions can be } \\
\text { analyzed }\end{array}$ & $\begin{array}{l}\text { CC } \\
(\text { small SP) }\end{array}$ & \\
\hline & & Label-free & $\begin{array}{l}\text { Cells with dark granules, such as } \\
\text { melanoma cells cannot be analyzed }\end{array}$ & & \\
\hline & & $\begin{array}{l}\text { Constant, gradient and oscillating } \\
\text { forces }\end{array}$ & Heat may affect measurements & & \\
\hline \multirow[t]{3}{*}{ Optical laser tweezers } & $\begin{array}{l}\text { Indentation using an optically trapped } \\
\text { bead }\end{array}$ & Relatively high cell numbers & $\begin{array}{l}\text { Single cells or small cell cluster without 3D } \\
\text { matrix environment }\end{array}$ & SC & $2 \mathrm{D}$ \\
\hline & Elasticity & & No generation of a 3D force trap & $\mathrm{CC}$ & $3 \mathrm{D}$ \\
\hline & Viscosity & & & & \\
\hline \multirow[t]{3}{*}{ Magnetic tweezers } & & Relatively high cell numbers & Cells need to be on the surface & SC & $2 \mathrm{D}$ \\
\hline & & $0.5-1$ cell per min & $\begin{array}{l}\text { Bead internalization may impact } \\
\text { measurements }\end{array}$ & $\mathrm{CC}$ & \\
\hline & & $\begin{array}{l}\text { Live cells without resorting to } \\
\text { intense irradiation, can be easily } \\
\text { multiplexed }\end{array}$ & $\begin{array}{l}\text { Cell movements not allowed during } \\
\text { measurements }\end{array}$ & & \\
\hline \multirow[t]{3}{*}{ Microrheology } & Elasticity & Time-dependent & Limited by modeling accuracy & SC & $2 \mathrm{D}$ \\
\hline & & & & $\mathrm{CC}$ & \\
\hline & Viscosity & Frequency-dependent & Computationally expensive modeling & $\mathrm{SP}$ & $3 D$ \\
\hline
\end{tabular}


TABLE 1 | (Continued) Most important characteristics of specific biophysical techniques.

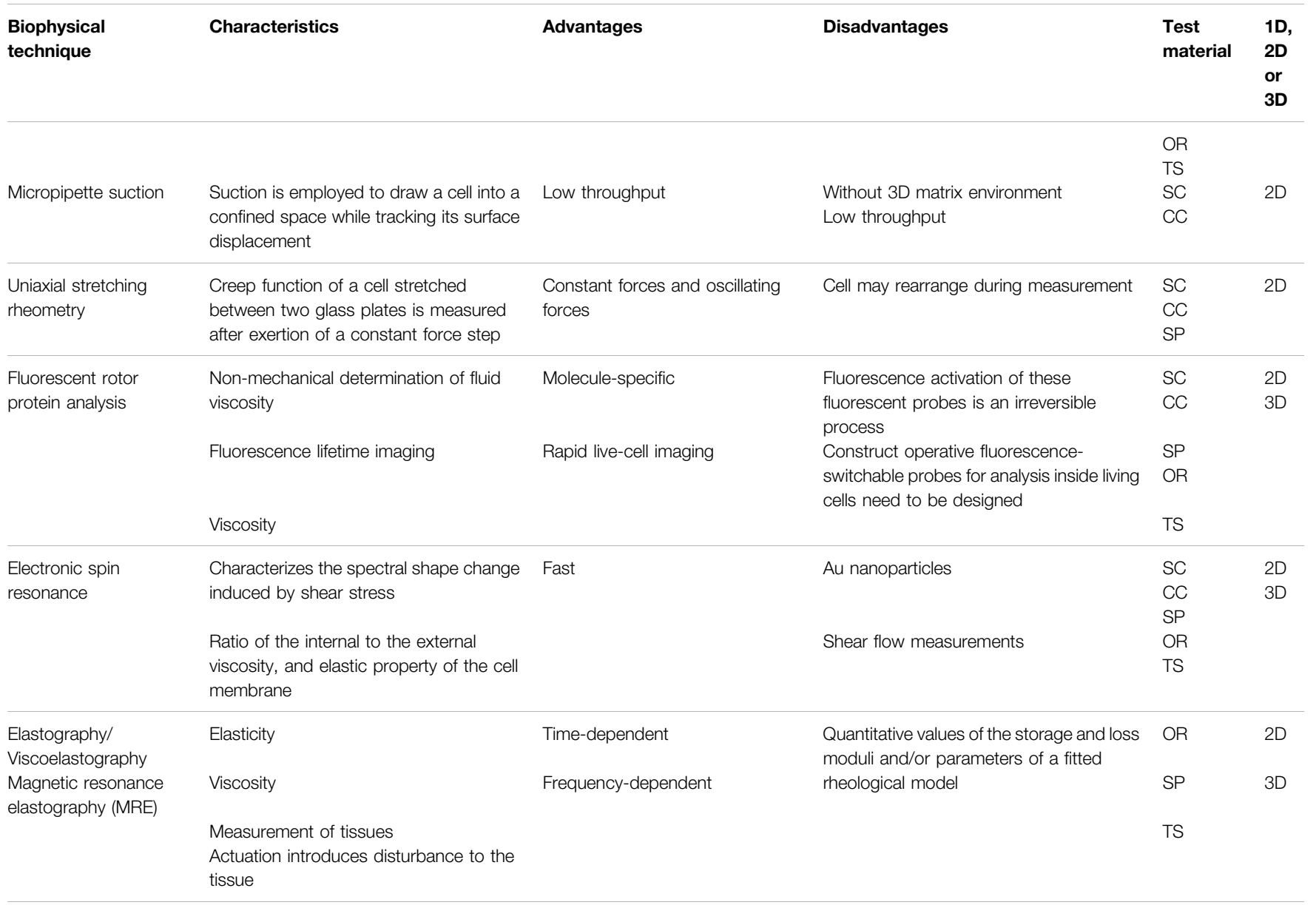

SC, single cells; CC, cell clusters; OR, organoids; SP, spheroids; TS, tissue specimen; 1D, one dimensional; 2D, two dimensional; 3D, three dimensional.

recent years, since it has become a research focus to seek for a correlation of viscoelasticity and the state of disease, differentiation of cells, or the transformation of cells or collections of cells (Darling et al., 2008; Lekka et al., 2012; Rother et al., 2014; van Zwieten et al., 2014). Therefore, AFM turned out to be a suitable technique for probing cell mechanics, including viscoelasticity in 2D and 3D model systems. However, AFM indentation continues to be a favorite method for exploring the nanoscale characteristics of soft samples such as cells, spheroids and tissues (Hutter and Bechhoefer, 1993; Gibson et al., 2005; Engler et al., 2009; Gavara, 2016). In AFM, the elastic characteristics of living cells are generally assessed by force-displacement (F-Z) curves. The Hertz model or its variations are imposed on the approximation section of the F-Z curve to obtain the Young's modulus, the elastic characteristic employed to define the mechanical performance of the specimen (Mahaffy et al., 2000; Pirzer and Hugel, 2009). Physical models such as these, however, hypothesize a purely elastic character of the specimen, whereas in fact the majority of biological specimens are viscoelastic. Viscoelasticity is evident in a marked hysteresis between the approach and retraction segments of the curves (Rebelo et al., 2013); the dependence of the indentation velocity on the E-Hertz values obtained from the force profiles with the Hertz model (Hertz, 1882); the force relaxation phenomena at constant indentation height and the creep at constant imposed force (Nawaz et al., 2012). If a standard F-Z curve might also be employed to quantify viscoelastic characteristics, it becomes feasible to adopt a standard method with properly quantified uncertainties (Sniadecki et al., 2007) for both viscoelasticity and elasticity determinations. Apparently, until now, this did not seem an option due to the absence of a mathematical/computational setting that enables post-processing of force-displacement measurements to exhaust the pertinent viscoelastic constitutive variables. A new methodology has been presented to derive the viscoelastic characteristics of soft specimens including cells, spheroids and tissues directly from standard AFM F-Z curves. This technique is founded on the wellestablished theoretical model pioneered by (Crick and Hughes, 1950) for the displacement constraint problem of a linear viscoelastic half-space through a rigid axisymmetric indenter under arbitrary load conditions. The Crick and Hughes model can be integrated with cognate numerical techniques to handle both approach and retraction phase datasets of the experimental F-Z curves (Efremov et al., 2017). The methodology is confirmed 
with finite element simulations, with experiments on polyacrylamide hydrogel specimens, and by benchmarking against in place AFM viscoelasticity measurement approaches.

Besides single biophysical technique approaches, a combined approach can be employed to further improve the mechanical characterization of cells. For instance, the pairing of an AFM with a confocal fluorescence lifetime imaging scanning microscope to examine the mechanical characteristics of individual adherent cells seems to be of high relevance in this context (Fuhrmann et al., 2011). As a result, force-indentation curves can be identified, and subsequently indentation depth-dependent elastic moduli have been obtained for several cell lines and different cell types. The stiffness tomograms show clear distinctions in the mechanical characteristics of the cell lines examined. This finding indicates that the microscopic interpretation of the enhanced compliance of cancer and precancer cells might reside in their proneness to "crumble and yield" rather than in their capacity to "bend and flex." Moreover, AFM is a high-performance method that can quantitatively map the mechanical viscoelastic characteristics in parallel with imaging the 3D topography of specimens. But it is restricted to adherent cells and can only measure from the upper surface of the cell. When oversimplified models are employed to derive an effective elastic modulus $\mathrm{E}_{\mathrm{eff}}$, the complex geometry and characteristically very inhomogeneous nature of the cells are entirely discarded, and it is frequently challenging to reconcile the elastic response values obtained in this manner with those acquired in other kinds of measurements.

In addition to analyzing the mechanical properties of adhesive cells, certain biophysical techniques such as the optical cell stretcher technique can also be used to analyze the mechanical properties of non-adherent cells. Two decades ago, the optical cell stretcher was invented, in which two counter-propagating divergent laser beams are pointed at a cell (Guck et al., 2001, Guck et al., 2005). When both beams share the identical intensity, there is no net force exerted on the cell lengthwise down the optical axis. However, it emerges that in tandem with a lateral optical gradient force that holds the cell on the optical axis, a symmetrical pair of forces affects the cell surfaces that stretches the cell in the optical axis direction. The cells will be captured in the center of the stretcher, and by simply applying different laser powers, the forces applied to the cell surfaces resulting in longitudinal stretching can be adjusted. From such deformation profiles, an effective cellular compliance J can be deduced, including timedependent characteristics. In view of viscous and elastic inputs, such measurements can be investigated using power law rheology models, a standard linear solid model, or a Burger model (Ekpenyong et al., 2012). Among the main benefits of the optical stretcher is the capability to couple it with microfluidic instruments and perform high-throughput measurements of cell compliance. Nevertheless, the distributions of optical deformability appeared to be so widespread that it was not practical to uniquely classify single cells (Guck et al., 2005). However this method is also suitable for a multitude of physiologically adherent and non-adherent cells, which can be performed on the one hand with the exclusion or on the other hand with the admixture of biochemical drugs that interfere with cell mechanics (Chan et al., 2015; Kunschmann et al., 2017, Kunschmann et al., 2019; Mierke et al., 2017, Mierke et al., 2020). In addition to the optical deformation of a cell, an important thermal impact on the specimen also arises, which permits the induction of fast temperature variations (Kießling et al., 2013). Leveraging this thermal phenomenon, optical stretching consequently facilitates a new category of investigations referred to as "thermorheology," which extends beyond simple mechanical stretching.

Another biophysical technique to probe the mechanical properties of adhesive cells, spheroids and tissues is magnetic tweezers. A totally different geometric attempt to investigate cells physically also leverages the interplay of magnetic particles with an external magnetic field. Perhaps the earliest record of the basic principle of magnetic tweezers brought to bear on biological systems was a 1950 experiment by Crick and Hughes, which it then referred to as the magnetic particle method (Crick and Hughes, 1950). In specific, cultivated chicken fibroblasts have been induced to phagocytose ferromagnetic particles. After imposing a magnetic field, three different types of movements of these particles could be perceived as twisting, pulling and pushing. These results indicate that the cytosol of cells is not only viscous but also possesses elastic characteristics. A crucial element in these experiments is the accurate calibration of the forces exerted on the typically superparamagnetic beads in the external rather less homogeneous magnetic field. Accurately monitored forces of up to $10 \mathrm{nN}$ on $4.5 \mu \mathrm{m}$ sized beads have been achieved and the mechanical response of adherent cells to the magnetic stimulation of the beads bound to plasma membranes has been determined (Bausch et al., 1998, Bausch et al., 1999).

Subsequently, superparamagnetic beads coated with integrin ligands have been employed to mechanically explore the coupling strength between the extracellular matrix scaffold and the cytoskeleton (Kollmannsberger and Fabry, 2007; Kollmannsberger et al., 2011; Mierke et al., 2017). Viscoelastic behavior can be determined by performing these creep experiments. However, the majority of experiments have been performed under 2D settings, where the cells adhered to a flat substrate, which can be coating with extracellular matrix protein for supporting cell adhesion and spreading. It is crucial in this experiment, however, to bring the magnet extremely close to the probe, which can be difficult for cells in $3 \mathrm{D}$ microenvironments or for tissues. Another disadvantage is that only adhesive cells can be measured and non-adhesive cells are generally excluded.

Nevertheless, it has been observed that these biophysical analyses may be heavily biased through the precise region of a cell being probed (Haga et al., 2000), changes in the cytoskeletal framework due to an imposed stress (Reed et al., 2008) or cell specific interactions occurring to a probe (Squires and Mason, 2010). All these interferences can distort determinations of cell viscoelasticity. Therefore, it was of great interest to develop other biophysical techniques that circumvent these shortcomings. A novel approach for developing such as biophysical measurement technique is presented in the following subsection.

\subsection{Novel Contact Free Measurement Technique of Viscoelasticity}

To determine stiffness, standard techniques (Table 1) involve the employment of cell contact or invasive probing and are therefore 
low throughput, work demanding, and constrained by placement of the sensor. Based on these findings, it seems to be obvious that another high throughput, less work load-based and sensorindependent technique needs to be engaged. Quantitative phase imaging (QPI) provided this, offering a probe- and contact-free approach to quantify variations, such as fluctuations, in the viscoelasticity of cells. In specific, QPI readings exhibit a pronounced underdamped reaction to temporal variations in the allocation of cell biomass (Nguyen et al., 2020). The effective stiffness and viscosity data obtained from these oscillations of the cell biomass mass distributions are related to the effective cell stiffness and viscosity determined with AFM. This finding is consistently true for different cell types with varying levels of cytoskeletal perturbation and throughout the epithelial to mesenchymal transition (EMT) of individual cells. Consequently, the QPI can be used to reliably and quantitatively determine the viscoelasticity of cells (Nguyen et al., 2020).

A QPI-based approach to accurately determine the viscoelastic properties of cells that is non-contact and non-invasive has been developed as a new technique referred to as quantitative phase rheology (QPR). Specifically, QPI (Popescu and Park, 2015) generally represents a microscopic approach to assess the phase shift or delay of light resulting from its interference with the relative dry matter or non-aqueous matter of a cell's biomass (Zangle and Teitell, 2014). By means of an experimentally ascertained cellaverage specific refractive index, the phase shift of the light can be correlated with the biomass of the cell (Barer, 1952; Davies and Wilkins, 1952). The growth of cells (Mir et al., 2011), cell death (Pavillon et al., 2012), and reactions to growth impairment through chemotherapeutics or targeted inhibitory substances of classical biological processes (Reed et al., 2011; Mir et al., 2014; Hejna et al., 2017) have been analyzed with QPI.

Moreover, previously QPR has been applied to determine the viscoelastic properties of the membrane of enucleated erythrocytes, that incorporated the design of an analytical model connecting the vibrational modes monitored with the viscoelastic characteristics by autocorrelation of the quantitative phase data (Popescu et al., 2008; Park et al., 2010). Since this model has been developed for enucleated cells, it may not be directly transferable toward the intricate complex nucleated cells. In applying the model to nucleated cells, spatial and temporal autocorrelations of quantitative phase data derived from colonies of human pluripotent stem cells evidenced both a more substantial amount of spatial coordination and a quicker rate of temporal decorrelation for pluripotent cells when compared to their more differentiated progeny (Zangle et al., 2013). More recently, it has been found that spatial autocorrelations of quantitative phase data provide an index of intracellular disarrangement of cells, a factor that is linked to cell stiffness induced in reaction to deformation caused by fluid shear (Eldridge et al., 2017). Still more work on QPR highlights that the temporal autocorrelation of quantitative phase data is associated with cellular transport behavior, encompassing diffusion (Wang et al., 2011; Ceballos et al., 2015; Kandel et al., 2017), and indicates a correlative association with cellular stiffness (Ma et al., 2016). At present, however, there is to my knowledge no other QPR technique (Nguyen et al., 2020) that can simultaneously model and accurately measure both the elastic and viscous moieties of cell viscoelasticity. More specifically, the autocovariance in time of the quantitative phase data for cells in the interphase of the cell cycle has been revealed to behave in a manner similar to a massspring-damper system (Nguyen et al., 2020). The elastic and viscous parts that characterize this behavior are linked to the viscous and elastic stiffness elements of interphase cells, which have been revealed through AFM analyses. To determine whether the mechanical properties rely on the actin cytoskeleton, the stiffness of three different cell types has been altered through addition of a pharmacological drug, such as cytochalasin B (Petersen et al., 1982), which impairs the polymerization of actin. Indeed, there is a high degree of correspondence between QPR outcomes and AFM viscoelasticity readings. To confirm that stiffness has a physiological function in cells of the same genetic origin during a cell state transition, QPR is applied to a cellular model of EMT (see also below) (Zhang et al., 2014). The hypothesis is supported by a novel EMT-based gene signature, encompassing ITGAV, DAB2, SERPINE1, MATN3, and PLOD2, that has been identified recently for gastric cancer (Dai et al., 2021). In line with expectations, the QPR measurements of stiffness and viscosity are related to EMT status (Nguyen et al., 2020). In addition, the results indicate that label-free QPR can be utilized to evaluate cell stiffness and viscosity, which confers an advantage over conventional biophysical techniques for examining the mechanical characteristics of cells and thus greatly broadens the use of QPI to monitor cell performance (Table 1).

Specifically, the locomotion of the cell biomass, when quantified as the autocovariance of the quantitative phase image measurements, exhibits a harmonically oscillatory movement. The oscillation and decay of the autocovariance can be addressed by employing a two-parameter viscoelastic model. The fitting of this model to empirical data permits the estimation of separate quantities for the effective stiffness and viscosity of a cell. However, earlier methods exist for measuring stiffness (Eldridge et al., 2017) using QPI datasets. The temporal measurements of the cell biomass movement (Nguyen et al., 2020) and other rheological features of a cell (Wang et al., 2011; Ceballos et al., 2015; Eldridge et al., 2017; Kandel et al., 2017) by QPI is what will be denoted as QPR.

To differentiate among various cell types, states, and operating conditions using the QPR measurements, a uniform stiffness over the measurement interval of approximately $5 \mathrm{~h}$ has been assumed. However, this hypothesis is not valid in mitosis, when the stiffness of the cell undergoes a fundamental alteration (Stewart et al., 2011). Hence, dividing cells need to be excluded from the analysis. In specific, an automated technique for identifying cell divisions has been developed to eliminate them from the quantitative phase image data (Nguyen et al., 2020). Thus, QPR can automatically handle QPI data from living cells and provide rheological characteristics of cells. Future efforts should be placed on the improvements in the spatial and temporal resolution of QPR that are necessary to assess the magnitude of viscoelastic alterations of cells throughout mitosis. These data may then aid to understand the functional role of cell mechanics in this specific process. 
QPR and AFM measurements provide cellular viscoelasticity within a similar force and time regime. An interpretation of the experimental data can be provided by a model presented by Qian (Qian, 2000) that has been developed for the tracking of single particles located inside a Kelvin-Voigt material. Qian's model offers comparable forecasts to the well-known classical series spring-damper-based Maxwell material model that can be performed on QPR measures. The Maxwell material model can be employed when the track shifts of small particles of cell biomass are effectively presumed when embedded in a Maxwell material. A moderate fit to AFM viscosity values $\left(R^{2}=0.81\right)$ can be attained when applying the Kelvin-Voigt model to QPR data sets relative to the fitting of the Maxwell model $\left(R^{2}=0.89\right)$. This implies that a Maxwell material model appears to be the more suitable twoparameter linear viscoelastic material model for the purpose of analyzing the QPR results. While this simple linear two-parameter model is a somewhat streamlined perspective on cell viscoelasticity, this model still accurately grasps the key attributes that have been delineated in the data.

Although this physical explanation of the mathematical model contains an inertia expression, it still reflects the response of a fluid exhibiting a low Reynolds number. This phenomenological hypothesis permits fitting a two-parameter viscoelastic model and deducing the rheological features of the cells based on the QPI data, which correspond to the AFM results. With respect to a possible physical significance of this expression, it has been demonstrated that inertia-like oscillations can arise in actively propelled, viscoelastic fluids (Berner et al., 2018). Since the cell remains an active composite (Chen et al., 2018), it can be hypothesized that the inertia-like performance exhibited in this system may result from a close association between viscoelastic material characteristics and active force generation due to cytoskeletal reorganizations. This points to the requirement for future models built on a superior cellular material model that can more fully accommodate these cellular mechanical characteristics.

Notwithstanding the fact that there is strong degree of correlation between AFM and QPR measures of stiffness and viscosity, a major disparity remains in the magnitude of these scores. Part of this discrepancy is accounted for by probe size disparities. Specifically, the AFM probe tip radius is $500 \mathrm{~nm}$, while the effective probe for QPR involves the stuff inside the cytoplasm. The noted variation in the magnitude of the QPR stiffness in comparison to the AFM stiffness value is approximately $10^{4}$, which indicates a QPR probe dimension of approximately $5 \mathrm{~nm}$. In addition, the cell can be modeled as a strictly linear viscoelastic material, but in broad terms, the cell rheology is a function of the length scale, strain rate, and amount of imposed force, which may vary between these two different kinds of approaches. In this way, AFM determines the viscosity from viscous displacement, while QPR provides an effective coefficient of friction that a particle experiences due to the viscosity of a cell. From a technical point of view, these are two distinct characteristics that are intimately linked by viscosity. The QPR approach is closest to passive particle tracking in microrheology (Weihs et al., 2006), which yields a stiffness value derived from the anticipated response to passive particle motion. The basic equations for the input and output factors of these three biophysical techniques are discussed briefly in the following. Fluorescently labeled sensor beads are embedded in a material, such as cells, and their Brownian motion is recorded with video microscopy. To link this particle movement to the rheological characteristics of the material, each sensor particle is monitored. After recording the movement of the sensor particles, the locations of the particles in each image are mapped according to their brightness-weighted centroid and then associated to form trajectories. The composite-averaged mean-squared displacement (MSD, $\left\langle\Delta r^{2}(\tau)\right\rangle$ is computed based on these particle trajectories. The MSD averaged over the entire composite provides a quantification of the particle movement and therefore reveals insight into the rheology and physical condition of the material, such as a cell. The particle movement is relatable to rheological characteristics, for example the creep compliance $J(\tau)$, applying the generalized Stokes-Einstein relationship (GSER). The GSER is given in Eq. 1:

$$
\left\langle\Delta r^{2}(\tau)=\frac{k_{B} T}{\pi a} J(\tau)\right.
$$

where $\tau$ stands for the lag time, $k_{B} T$ denotes the thermal energy, and $a$ indicates the radius of the particle.

The GSER is applicable in cases where several conditions are fulfilled: firstly, the length of the probe particle is much longer than the characteristic length span of the material, secondly, effects of inertia on the sensor probe and the fluid are ignorable, and thirdly, the length compression factor of the fluid is ignorable (Squires and Mason, 2010; McGlynn et al., 2020). AFM techniques evidenced that the elastic modulus of the lower invasive epithelial bladder cancer cells RT112 displayed a plateau modulus at the slower frequencies, which is not the case for the two other stronger invasive epithelial bladder cancer cells T24 and J82, implying that the invasiveness renders the cells to be less elastic (Abidine et al., 2021).

When the applied force is less than $3 \mathrm{nN}$ to stay in the linear elastic range, the Hertzian model can be employed. When the tip of the cantilever comes into physical contact with the specimen, the force imposed on the cantilever rises to a pre-selected nominal value $F_{0}$, that is equivalent to an initial indentation $\delta_{0}$. The correlation is provided by Sneddon's modification of the Hertzian model of contact mechanics, as indicated in Eq. 2 below:

$$
F_{0}=\frac{3 E \tan \Theta}{4\left(1-v^{2}\right)} \delta_{0}^{2}
$$

$E$ denotes the Young's modulus of the cell, $v$ its Poisson ratio of approximately 0.5 and $\Theta=20^{\circ}$ represents the half pyramid angle. $\delta_{0}$ is selected in such a way that the indentation depth of the tip into the specimen is sufficiently deep to have a suitable contact area and never too deep to stay within the range of linear elasticity.

Moreover, microrheology data (Wirtz, 2009) compared to AFM data (López-Fagundo et al., 2016) on mouse embryonic fibroblasts reveal large discrepancies in the magnitudes measured: $14 \mathrm{~Pa}$ for microrheology stiffness compared to $7.7 \mathrm{kPa}$ for AFM stiffness. A similar mismatch between AFM stiffness and microrheology stiffness values has also been identified for MCF10A mammary epithelial cells (Li et al., 2009). 
Collectively, these findings demonstrate the inherent capability of a label-free and non-contact technique that can accurately gauge the rheological behavior of cells. As a transmitted light microscopy approach, QPI is non-invasive and therefore it minimizes the interfering impact of probes in the examination of biological events in living cells. QPI can be deployed to obtain a measure of the biomass distribution of cells over time. Notably, the autocovariance of this biomass distribution over time, referred to as $C \varphi \varphi$, is calculated to quantify variations in the biomass distribution driven by the movement of cell structures or compartments, such as organelles.

To assess the resemblance of the quantitative phase data over time, an unbiased estimation of the autocovariance of the phase shift signal can be employed, representing an autocorrelation of the data that have been subtracted from the mean. The temporal autocovariance is standardized to the total amount of data points taken in each autocovariance window, with respect to the end of the time shift window $t_{0}$, and defined in Eq. 3:

$$
C_{\varphi \varphi}\left(x, y, t_{0}, \tau\right)=\frac{w \sum_{j=0}^{w-\tau / \Delta t}\left(\varphi\left(x, y, t_{o}-j \Delta t\right)-\varphi\left(x, y, t_{0}\right)\right)}{\left(w-\frac{\tau}{\Delta t}\right) \sum_{j=0}^{w-\tau / \Delta t}\left(\varphi\left(x, y, t_{0}-j \Delta t\right)-\varphi\left(x, y, t_{0}\right)\right)^{2}}
$$

Additionally, under the assumption of damped oscillations that rely on a series of harmonics a and b can be described as in Eqs 4, 5, respectively:

$$
\begin{gathered}
a=\frac{k}{2 \mu} \\
b=\left(\frac{k}{m}\right)^{1 / 2}\left(1-\frac{k m}{4 \mu^{2}}\right)^{1 / 2}
\end{gathered}
$$

wherein $k$ denotes the effective spring constant of the cell sensing the particle over the measurement interval, $\mu$ stands for the effective damping coefficient resulting from the viscous forces of the cell sensing the particle, and $\mathrm{m}$ represents the average biomass of the particles in the system. The autocovariance equation can be broken down and consequently, the effective stiffness can be presented by the relation of the fitting coefficients as denotes in Eq. 6:

$$
\frac{k}{m}=a^{2}+b^{2}
$$

Since QPR relies on an established quantitative phase imaging workflow, QPR can be amenable to full incorporation into other types of measurements that are prevalent in quantitative phase techniques, including cell biomass or biomass accumulation rates.

\section{MOLECULAR MECHANOSENSORY BEHAVIOR}

The molecular mechanosensory behavior of cells relies firstly on their interplay with neighboring cells and secondly on their interaction with the extracellular matrix environment. Cells must perceive their environment and be able to react to changes. Thereby, the cells can even adapt their cellular functions, such as their adhesion and migration capacity. Mechanical cues can be perceived by cells through a wide variety of membrane-anchored receptors, comprising stretchactivated ion channels, integrins, cell membrane-spanning G-protein-coupled receptors and cadherins (Paluch et al., 2015). The integrin- and cadherin-based adhesion complexes form at the contact interfaces between cells and extracellular matrix and between cells and cells, accordingly. Both involve proteins that respond to alterations in tensile forces and adjust their molecular composition and dynamics in reaction to these forces, leading to biochemical signaling effects that relay the mechanical input (Han and de Rooij, 2016; Martino et al., 2018). Multiple integrins join together to form adhesion complexes known as focal adhesions, which transfer mechanical forces bidirectionally across the extracellular matrix and the intracellular actomyosin cytoskeleton (Geiger and Yamada, 2011; Jansen et al., 2017). The involvement of integrins (particularly integrin a5 $\beta 1$ ) in focal adhesions during mechanotransduction is clearly evident (Mierke et al., 2011; Sun et al., 2016; Jansen et al., 2017; Elosegui-Artola et al., 2018). Moreover, these mechanical signals govern a variety of cellular processes that exploit a repertoire of mechanosensors converting forces into biochemical pathways with mechanotransduction (Box 1).

\subsection{Molecular Mechanosensory Interplay Between Cells of the Same Cell-Type: Dynamics of Adherens Junctions and Cadherins}

Within the major elements of adherens junctions and desmosomes are molecules of the cadherin family (Rübsam et al., 2018). Among them are cadherins of the classical-type, such as E-cadherin, desmoglein and desmocollin that can be found in epithelial cells (Neel et al., 2021) and clustered protocadherins with $\alpha, \beta$ and $\gamma$ subfamilies (Nicoludis et al., 2016). The latter play a role in neural adhesion and selfrecognition. Classical cadherins, such as E-cadherin (epithelial cells) and VE-Cadherin (endothelialc cells) belong to transmembrane glycoproteins that possess an extracellular domain driving cell-cell adhesion through homophilic or heterophilic interference and an intracellular domain regulating signal transduction cascades associated in a variety of cellular functions and processes, encompassing polarity, and gene expression (Meng and Takeichi, 2009; Liu et al., 2010; Baum and Georgiou, 2011; Maruthamuthu et al., 2011; Leckband and de Rooij, 2014; Ravasio et al., 2015). The positioning, adhesion strength, and distribution of adherens junctions relies on the specific context, such as the specific cadherin related to a distinct cell type. For example, E-cadherin in adherens junctions of epithelial cells, VE-cadherin in adherens junctions of endothelial cells and $\mathrm{N}$-cadherin in adherens junctions of multiple cells, including cardiac muscle cells and mesenchymal cells. The cell adhesion molecule $\mathrm{N}$-cadherin represents a biomolecule marker for the EMT, which leads to the 
BOX 1 | Definitions and Terminology.

Cytoskeleton = A scaffold of biopolymer fibers that fill the entire cell. It is the main contributor of the material reaction of the cell that is deformed or under stress Compliance $(\mathbf{J})=$ The relative degree to which a body yields to deformation by a force, typically sampled as time-dependent strain divided by constant stress.

Deformation (of cells) $=$ Cells possess the ability to change their shape in response to mechanical stress by remodelling their shape.

Durotaxis $=$ refers to the directional cell movement in which cells detect a gradient of varying stiffness (rigidity) in their microenvironment and preferentially migrate toward the stiffer extracellular matrix.

Extracellular matrix scaffold = The extracellular matrix represents a non-cellular moiety that forms the material support backbone for cellular elements. Beyond its structural nature, it has an important, active function in morphogenesis, differentiation, and homeostasis.

Elastic modulus $=$ Otherwise referred to as the Young's modulus, the E value provides a quantification of the strain reaction to a uniaxial stress in the direction of that stress in the linear range.

Elasticity $=$ The characteristic of a material to deform to a certain degree under the action of a force and to recover its initial shape after the force has been withdrawn. Elasticity in itself is not a measurement of stiffness; what is generally intended is the elastic modulus, the ratio of stress to strain for a completely elastic solid.

Entanglement (of fibers) = Polymeric fibers can be wrapped around one another without being physically connected.

Hysteresis $=$ The term describes the dependence of the state of a system on its history.

Jamming = Material viscosity becomes more divergent as the particle density rises.

Linear elasticity = The Young's modulus or shear modulus is constant over spectrum of strains; thus, the stress is proportional to the strain.

Mechanotransduction = It is a process in which cells sense and react to mechanical cues by converting them into biochemical cues that drive specific cellular reactions.

Non-linear elasticity $=$ Young's or shear modulus that alters due to strain.

Phase transition = Macroscopic alteration of the characteristics of a system (order parameter) when a parameter exceeds a specific critical level (control parameter), also known as a critical point.

Poroelasticity = Investigation of the interaction of a porous elastic array with an interpenetrating pore fluid in a poroelastic material.

Strain $=$ The value denoted by $\gamma$ quantifies the deformation of a body. Specifically, it provides a quantification of the relative displacements of components of the body that are not accounted for by the movements of the rigid body. (unitless parameter that quantifies the amount of deformation after the exertion of stresses).

Strain (longitudinal) $=$ represents the fractional alteration in length or elongation: $\varepsilon=\delta / L$

Strain stiffening response $=$ The nonlinear reaction of multiple biomaterials is the rise in stiffness with the augmentation of strain.

Stress $=$ Force is per unit area: $\sigma=\mathrm{F} / \mathrm{A}$, whereby the SI unit is $\mathrm{N} / \mathrm{m}^{2}$.

Tension $=$ is the pulling force transmitted axially by the means of a string or similar object.

Viscoelasticity = A combination of elastic and viscous responses to applied stress. Most biological materials are viscoelastic: when they are deformed, the degree of their resistance decays with time, usually to a stable baseline (viscoelastic solid) but sometimes to zero at long times (viscoelastic fluid).

Viscosity = The quantity denoted by $\eta$ quantifies the flow of the material at a specific velocity under load (Measure of the resistance of a liquid to deformation as a reaction to a shear stress. Viscosity is the relationship of stress to strain rate of a fluid).

Young's modulus $=\mathrm{A}$ constant that expresses the resistance of a material to deformation when stretched: $\mathrm{E}=\sigma / \varepsilon$.

establishment of an aggressive and invasive cancer cell phenotype through a so-called cadherin-switch that is subsequently associated with a transformation of a non-motile (epithelial) to a motile (mesenchymal) phenotype (Wheelock et al., 2008; Gheldof and Berx, 2013). The earliest discovery of cadherins started in cultures of epithelial cells, where type-I cadherins, such as E-cadherin, exhibited cells of epithelial origin with strong adhesions and its expression has been governed by stalled rather than motile cellular phenotypes.

Due to the specific environment adherens junctions behave either highly dynamical or extremely stalled and these dynamical remodeling can be adapted at every single step of the assembly of adherens junctions. Adherens junctions assembly in three major steps. Firstly, cells attach to one another in an initial step where extracellular domains of cadherins are involved, with the type of cadherin being displayed by neighboring cells and facilitating the tethering strength of this particular step. A second step represents the lateral extension of a nascent adhesion that covers the additional engagement of cadherins to enlarge the interaction zone. A third step encompasses the stabilization of the adherens junctions, where the cytoskeletal activity of collectively migrating cells are tuned and guided (Cavey and Lecuit, 2009; Meng and Takeichi, 2009; Theveneau and Mayor, 2012; Barriga and Mayor, 2015). Whereas the type of cadherin, such as E-cadherin, VEcadherin or N-Cadherin, exhibited by a specific cell type may be important in shaping the strength of adhesion in the initiation phase, the conversion of cadherins is essential in governing the dynamics and durability of lateral extension and stabilization of adherens junctions, and thus the lifetime and vigor of the connection. Cadherin levels, such as E-cadherin (epithelial state) and $\mathrm{N}$-cadherin (mesenchymal state), can be regulated at the transcriptional scale through specific transcription factors, including members of the Snail, Twist, and ZEB families, as an element of a specific switching process referred to as EMT, and the conversion of these adherens junctions proteins is subject to post-translational modifications. Downregulation of E-cadherin results in loss of stable epithelial cell-cell adhesive junctions, such as adherens junctions, apico-basal cell polarity, and the architecture of epithelial tissue, which aids in the escape of cancer cells from the primary cancer hub (Perl et al., 1998; Kourtidis et al., 2017). In contrary to the anti-migration function of E-cadherin, N-cadherin confers increased migratory and invasive capacity toward cancer cells independent of E-cadherin expression (Hazan et al., 2004). Thus, it seems that the procurement of $\mathrm{N}$-cadherin is a crucial pace in the metastasis of epithelial cancer and the advancement of the disease. In addition, the cadherin switch in collections of cells leads to an unjamming to jamming transition through the weakening of cell-cell interactions, such as adherens junctions (Ilina et al., 2020). There is also an E-cadherin-integrin crosstalk that govern the migratory capacity of cells, such as cancer cells (Canel et al., 2013), since the formation of an adhesome (Horton 
et al., 2015, Horton et al., 2016) may alter the E-cadherindependent cell-cell interaction and subsequently cellular locomotion. In addition to integrin-dependent migration, integrin-independent migration is also observed (Schmidt and Friedl, 2010). However, it can be assumed that both types of invasion are highly dependent on integrin-driven adhesion toward the extracellular matrix, whereas collective invasion also demands dynamically remodeled cell-cell adhesions, meaning that slackening of cell junctions is necessary for invasion to occur.

\subsection{Molecular Mechanosensory Interaction Between Different Cell Types in Transmigration}

\subsubsection{What Affects the Transmigration of Cells in a 3D} System?

These materials are selected primarily for their semipermeable and size-excluding characteristics to limit or permit transmigration and cell-cell coupling (Casillo et al., 2017). Nevertheless, previous investigations have demonstrated that pore size, pitch, and orientation influence cell performance, encompassing extracellular matrix production and migration (Irvine et al., 2001). The extracellular physical arrangement of integrin ligands in patterns of clusters may aid in the bunching of bound integrins into clusters, thereby perhaps governing cellular responsiveness to a specific average quantity of a ligand within the extracellular environment (Irvine et al., 2002). The mechanism underlying this response is only partially identified (Allahyari et al., 2020). Specifically, the 3D scaffold of the extracellular matrix environment can affect the migratory capacity of cells, when the pore size, adhesiveness or matrix stiffness is altered independently of each other (Peyton et al., 2011). It has been found that the greatest chance of substantial cell migration through the pores appeared at an intermediate pore diameter, and not at the maximum pore diameter, when it surpassed the cell diameter. Importance of co-culture systems, barrier systems, and organ-on-a-chip investigations (Zervantonakis et al., 2012; Jeon et al., 2015) arises from their utility in disease models and drug discovery, and from insights into cell-cell interactions at tissue boundaries (Bhatia and Ingber, 2014; Chung et al., 2018). There are interactions of cells with their surrounding extracellular matrix that are based on the pure structure, but there are also indications that this is not enough, which means that $3 \mathrm{D}$ matrices are additionally crucial for cell sensing mechanisms and bidirectional cell-matrix interactions. In some investigations, the structural purpose has been focused. The purpose of one investigation has been to disentangle the action of pore edges and pore openings on a porous membrane using a non-soiling, microstructured substrate (Allahyari et al., 2020). Therefore, a non-fouling micropattern has been generated on a silicon dioxide $\left(\mathrm{SiO}_{2}\right)$ substrate, which has a shape, dimension, and discontinuity profile similar to the pore openings of a porous membrane, but with no pore edges. The $3 \mu \mathrm{m}$ diameter pore size has been selected since it is a frequently employed pore size for membranes in the barrier modeling and it is sufficiently sized to accommodate leukocyte transmigration (Allahyari et al., 2020), whereas it is sufficiently small to impede endothelial cell transmigration in principle (Kukulski et al., 2007; Prabhakarpandian et al., 2013; Takeshita et al., 2014; Casillo et al., 2017; Chung et al., 2018; Salminen et al., 2019). To generate the non-fouling regions in the patterned substrate, poly(L-lysine)-g-poly(ethylene glycol) (PLLg-PEG) was used, which has been proven to be a proper candidate for producing these types of non-fouling patterns (Michel et al., 2002; Falconnet et al., 2004; Lussi et al., 2006; Marie et al., 2007; Azioune et al., 2010; Vignaud et al., 2012; Rothenberg et al., 2015; Liu and Yang, 2017). The PLL backbone of this polymer facilitates an efficacious adsorption on negatively charged surfaces such as $\mathrm{SiO}_{2}$, whereas the polyethylene glycol (PEG) branches impede cell adhesion to the coated substrate (Michel et al., 2002; Falconnet et al., 2004; Azioune et al., 2010). Microcontact stamplography and deep UV laser deposition are widely practiced chemical structuring techniques for the engineering of such a micropatterned surface substrate. However, as with any patterning technique, there are certain constraints, such as poor reproducibility due to stamp wear and poor resolution caused by imperfect polymer removal (Csucs et al., 2003; Azioune et al., 2009, Azioune et al., 2010; Musaev et al., 2011; Ligon et al., 2017; Tewary et al., 2019; Tenje et al., 2020). Photolithography and simple surface adsorption of the PLL-gPEG polymer could be successfully coupled to fabricate a reproducible pattern with less steps and without the abovementioned intricacies, thus providing high-resolution micropatterning (Casillo et al., 2017). The resulting microstructured substrate has been applied to examine the fibrillogenesis of fibronectin, migration characteristics, and spreading of endothelial cells. Endothelial cells have similar tendencies in fibrillogenesis of fibronectin and migration speed as previous results on porous substrates, but there were discrepancies in cell spreading and a smaller augmentation in migration speed on these substrates compared with previous findings on micropores. These results lead to the hypothesis that, in addition to the disruptive nature of the open pores, there are other physical drivers that lead to these slight variations in the behavior of the micropores.

\subsubsection{Effect of Interstitial Flow on Transmigration}

Besides structural cues, also non-structural effects impact the migratory phenotype of cells. For instance, the interstitial flow can be mimicked within these microfluidic chambers that impact cellular mechanical properties and motility, such as the directional migration along the streamline (Polacheck et al., 2017). Apart from this tri-culture assay, solution and surface chemical gradients can be generated within microfluidic chambers (Jeon et al., 2000; Kim et al., 2010). These microfluidic chambers for co-culture of cancer cells with endothelial cells can also be utilized for tumor spheroids (Ko et al., 2019) and therefore represent an intricate experimental platform for analyzing the migratory capacity through extracellular matrix scaffolds and endothelial barriers. There are also organ-on-a-chip models that fully grasp the $3 \mathrm{D}$ microenvironment of cancer cells (Glaser et al., 2022). For example, the immune microenvironment of cancer cells has been analyzed employing these organ-on-a-chip technologies 
(Yoon et al., 2020). Long-term studies are possible without leading to an excess of cell death and less growth factors need to be supplemented. In addition to the aforementioned advantages of $3 \mathrm{D}$ cultures, one disadvantage of $3 \mathrm{D}$ cultures is that manual handling of the organoids or microtissues and culture medium can be difficult if the microtissues are freefloating, breakable, or when physical access to the tissues is impeded by ambient containers and engineered devices. Some engineered devices ease the management of spheroids by confining them in plugged conical cavities, in perfused compartments in organ-on-chip constructs, or by embedding magnetic nanoparticles (Haisler et al., 2013; Kim et al., 2015).

\subsubsection{Impact of Matrix-Mechanics on Transmigration and Cell-Cell Junctions}

In a specific type of migration, which is referred to as extravasation (transmigration) of cells through an endothelial cell layer, a stiffer matrix, whatever the mechanism of stiffening, has been found to augment leukocyte-endothelial interactions in the inflammatory pathway. When endothelial cells are grown into a confluent monolayer on stiffer substrates, leukocytes can traverse the monolayer more efficiently than when the monolayer is built on a compliant medium (Huynh et al., 2011; Tao and Sun, 2015). The amplified transmigration has been extensively accounted for by a breakdown in endothelial cell-cell junctions rather than altered expression of inflammatory mediators in endothelial cells; but there is emerging indication that certain cells on stiffer substrates are more susceptible to inflammatory cytokines. Thus, endothelial cells exposed to tumor necrosis factor- $\alpha$ (TNF- $\alpha$ ) and thrombin show a stronger rise in tensile forces on stiff matrices in comparison with more compliant matrices (Urbano et al., 2017). Similarly, fibroblasts on stiff substrates are also found to be more responsive to TNF- $\alpha$ (Liu et al., 2015b; Southern et al., 2016), which enhances their spreading and generation of tensile forces. The exact mechanism whereby such elevated sensitivity arises is not understood, although it is probably attributable to an interaction with integrin-related signaling pathways.

However, the stiffness range is the crucial factor. Endothelial cell monolayers constituted more mature cell-cell junctions on soft substrates relative to glass, reflecting enhanced retention of vinculin and F-actin. Endothelial cell monolayers aided transendothelial migration on soft matrices (Mierke et al., 2008). Specifically, the mode of transmigration, such as transmigration between two neighboring cells or transmigration through the cytoplasm of a single cell, is regulated by the stiffness of the substrate. For example, immune cells, comprising peripheral blood lymphocytes and natural killer cells, exhibited a declining incidence of paracellular (between two neighboring cells) transmigration events with reducing substrate stiffness, whereas the incidence of transcellular (through the cytosol of an individual cell) events among peripheral blood lymphocytes enhanced (Onken et al., 2014). In line with this, melanoma cancer cells exhibited elevated transmigration with lower stiffness. Whether the viscoelastic properties of the substrate and of the endothelial cells play an additional regulatory role still needs to be figured out.
When cells, such as cancer cells, transmigrate through the endothelial monolayer, they seem to exert forces on. It is know that force application to the endothelial adhesion molecule PECAM activates GEF-H1 and LARG (Collins et al., 2012), whereas force application to endothelial ICAM-1 activates only LARG (Lessey-Morillon et al., 2014). Additionally, tension on JAM-A in endothelial cells leads to the activation of GEF-H1 and p115RhoGEF, whereas LARG is not activated (Scott et al., 2016). Consequently, when force is applied to cells various cell adhesion molecules govern the activation of one or two of a group of GEFs, encompassing GEF-H1, LARG and p115RhoGEF. It remains to be determined whether the variations are attributable to distinct adhesion molecule composites or to variations across cell types and traction modes. However, it has been shown that the mechanical properties of endothelial cells, such as their stiffness and fluidity are altered (Burridge et al., 2019).

The formation and sustenance of the majority, perhaps all, of animal tissues and organs is governed to a certain degree through the action of mechanics (Engler et al., 2006; Felsenthal and Zelzer, 2017; Urner et al., 2018). The focal adhesion protein talin and integrins perform pivotal tasks in the perception of and reaction to mechanical forces. Specifically, cells feel the rigidity of the extracellular matrix and the tissue strain conveyed from the extracellular matrix through integrins (Elosegui-Artola et al., 2018; Sun et al., 2016). Both of these cases increase the tension on the extracellular matrix integrin-cytoskeleton connection, resulting in stronger integrin bundling and increased signaling performance. Comprehension of these events is imperfect, but the key general principle is that both strain and rigidity alter the amount of stress present within the integrin-cytoskeleton junction, thereby changing the conformations and interactions of the proteins concerned. The core of mechanotransduction is how mechanical force is translated into a biochemical alteration, for example, the level of an effector at the adhesion site or the post-translational alteration of proteins.

\subsection{Molecular Mechanosensory Interaction of Cells and Their Matrix Environment}

It is well acknowledged that $2 \mathrm{D}$ cultures suffer from multiple constraints, such as disruption of interactions between the cellular and extracellular surroundings, alterations in cell morphology, polarity, and division pattern (Weaver et al., 2002; Mseka et al., 2007; Kapałczyńska et al., 2016). All of these alterations seem to impact the viscoelastic behavior of cells, spheroids and organoids. These drawbacks led to the emergence of models that can better mimic in vivo settings. $3 \mathrm{D}$ culture is this kind of technique. Within $3 \mathrm{D}$ culture setting, the storage and liberation of growth factors or cell surface receptor ligands or matrix-degrading enzymes and their regulatory molecules is possible compared to $2 \mathrm{D}$ culture systems. In addition, the diffusion of substances through a $3 \mathrm{D}$ matrix environment seems to be different and is likely to be hindered. The geometry and topology of the 3D matrix environment farther alters the cellular phenotype and consequently cell function. In agreement with this, the 
migration of cells can be impaired by a dense 3D matrix scaffold that cannot be broken down enzymatically by cellular substances or mechanically through bond rupture by cellular forces. Therefore, choosing the most adequate cell culture techniques in the field of cancer research may permit a better comprehension of cancer biology and thus optimize radio- and chemotherapy or discover innovative therapeutic options (Aggarwal et al., 2009). $3 \mathrm{D}$ culture tests represent a major advantage as they can preserve organotypic functionality as extensively as feasible. A simple approach is to employ completely differentiated cells that are directly prepared from living tissues, since they are considered to be in a native phenotype (Zuppinger, 2019).

In the historical context, 3D cultures have been first utilized systematically for pharmaceutical testing in cancer biology, explained in a part due to the presence of cellular aggregates containing a hypoxic core that share many resemblances with avascular solid cancers (Inch et al., 1970). It has been consistently shown that solely $3 \mathrm{D}$ technologies using co-cultures are capable of mimicking pivotal features of phenotypic and cellular heterogeneity, and microenvironmental facets of cancer growth (Thoma et al., 2014).

\subsubsection{D vs 3D Culture Conditions Affect the Cellular Mechano-Phenotype}

The $2 \mathrm{D}$ vs $3 \mathrm{D}$ culture conditions have been seen to be different in cardiomyocytes. The mechanical properties of cardiomyocytes have been identified to be heavily reliant on the composition and organization of the matrix, and time in cell culture. Cells appeared to stiffen and relax less in the first 3-5 days in cultivation prior to achieving a plateau in their mechanical characteristics. After the fifth day, cells on aligned matrices tended to be stiffer compared with cells on unaligned matrices, and cells on fibronectin matrices seemed stiffer compared with cells on collagen matrices (Deitch et al., 2012). In contrast, cardiomyocytes are subjected to a plethora of biochemical, mechanical, electrical, and other types of irritants that result in adequate reactions and finely tuned alterations in gene expression (Zuppinger, 2019). In addition, they also sense the shear stress generate through the blood flow. All of which is also applicable to other cells types, such as cancer cells or endothelial cells.

The overwhelming number of biological tissues have the capacity to distort and adjust to their new environment when faced with defiance from physical forces. Cells change their perceptual response depending on the microenvironment, as the cellular response to micrometer and submicrometer scale columns/pillars is fundamentally different (Ghassemi et al., 2012). In addition, depletion of rigidity sensing modules can elevate the growth of cancer cells (Yang et al., 2020). Thus, the precise interplay between intracellular elements of cells and environmental cues is critical for regulating cellular mechanotransduction that guides cell growth and thereby prevents excessive growth of cancer cells (Yang et al., 2020). Cells clad on 2D or 3D microstructured surfaces are prone to match their form to that imprinted on the pre-patterned surface structure (Théry, 2010). The question still remains unanswered as to whether the surface disturbance caused by pore opening is the only factor contributing to the alteration of the migration behavior of cells on a porous membrane, or whether the pore edges should also be considered as a decisive factor in the regulation of cell migration and spreading on a porous membrane. Pore edges, which can provide increased vertical contact area for cell adhesion and migration, have not been previously investigated as a separate contributing factor in studies involving porous membranes. Additionally, there is a growing interest in utilizing microposts to mimic cellular microenvironments in terms of mechanical cues. These studies demonstrate how flexibility of microposts can regulate cell spreading and migratory behavior of cells through modulating substrate rigidity (Allahyari et al., 2020; Yang et al., 2017; Zhong et al., 2020). However, similar to the role of pores in porous membranes, pillar walls can be considered as potential gripping points for cells, and this topological aspect of microposts can influence cell migration and spreading in addition to the substrate rigidity effect (Bettinger et al., 2009; Irvine et al., 2001, Irvine et al., 2002). Therefore, it is important to study pore edges or pillar walls as a contributing factor for the aforementioned changes in cellular behavior, particularly on porous membranes which are employed in an ever-increasing number of experimental examinations.

\subsubsection{Structural Cues Impact Cell Migration}

Moreover, during migration in vivo, cells generally prefer to squeeze through exceptionally narrow extracellular gaps, and in an effort to accommodate these intricate geometries, they extensively misshape (Théry, 2010; Lange and Fabry, 2013; Calero-Cuenca et al., 2018). Although the extent of distortion exhibited by cells or tissues can differ according to the time or nature of the tissue sampled, the majority of biological tissues act as nonlinear viscoelastic materials when subjected to physical stresses. A rather simplified meaning of viscoelasticity of biological matter is that the identical tissue behaves viscously and elastically when mechanically strained (Burstein and Frankel, 1968; Kucharová et al., 2007; Pajic-Lijakovic and Milivojevic, 2017). In general, biological matter, such as living tissues, have not commonly been investigated as non-linear viscoelastic materials. As mentioned before, pioneering work in mechanobiology treat biological tissues as purely and simplistic linear elastic materials, whereby Hookean behaviors is hypothesized when it is subject to mechanical deformation. As emphasized in this review, this view has changed, since biological materials have solely been seen to explore elastic characteristics when a temporal element is not included. However, when addressing time as an independent element in experimental setups, the curves displaying the reaction of biological matter to external mechanical deformation would become non-linear characteristics, when related to a control curve representing a "Hookean material" (Burstein and Frankel, 1968). This nonlinear mechanical reaction of biological systems has lately been reexamined and proven by utilizing purified and in vitro reconstituted cellular compounds. It assists in elucidating why several biological materials, including cells, undergo stiffening when stretched to prevent large deformations and preserve the intactness of the tissue (Goldenberg and Goldhirsch, 2005; Storm et al., 2005; Pogoda et al., 2014; van Helvert and Friedl, 2016). 
Therefore, a comprehensive framework has been established in which the response of biological materials to deformation is divided into, firstly, short-term linear-elastic behavior, which is an instantaneous, almost time-independent response, and secondly, time-dependent viscoelastic response. The latter can be considered at different time scales and structural levels, such as short-term subcellular, mid-term cellular, and long-term supracellular regimes (Thoumine and Ott, 1997; Bausch et al., 1999; Vicsek and Zafeiris, 2012; Pajic-Lijakovic and Milivojevic, 2017). Although the short- and medium-term regimes are in fact well recorded, the observation of a long-term supracellular viscoelastic regime is relatively less examined (Pajic-Lijakovic and Milivojevic, 2017). While individual cells are able to quickly reorganize cytoskeletal elements and react to stress (Mitrossilis et al., 2010), clusters of cells may take longer when reacting in a coordinated manner to environmental stress. Probably owing to the time delay caused by the demand for higher coordination of these cytoskeletal redistributions (Pajic-Lijakovic and Milivojevic, 2017). The advantage of $2 \mathrm{D}$ culture conditions are the high throughput and the capability of addressing intracellular structures impacting mechanical cues in more detail, due to the less working distance required and non-disturbed imaging quality through matrix scaffolds surrounding the cells. A major disadvantage is that the viscoelasticity can be altered through the viscoelasticity of the $3 \mathrm{D}$ matrix environmental scaffold that acts on cellular mechanical characteristics.

The cross-talk between human cancer cells, such as breast cancer cells, human endothelial cells and human tissue parenchyma, such as osteo-cell-conditioned extracellular matrix to mimic bone tissue, can be deciphered using $3 \mathrm{D}$ microfluidic tri-culture systems (Bersini et al., 2014). It has turned out to be useful in determining whether breast cancer cells migrate more efficiently into osteo-cell-conditioned matrices compared to non-conditioned matrices. These findings have enlightened the process of cancer cell dissemination to targeted tissues or organs.

In the following section, cellular components involved in regulating the viscoelastic response of cells and tissues are introduced together with their reaction to deformation and specific selected mechanisms that govern the dynamical processes of these cellular elements.

\section{REGULATION OF CELLULAR CONSTITUENTS, FOCAL ADHESION, CYTOSKELETAL AND NUCLEAR COMPONENT ASSEMBLY, RESTRUCTURING AND TURN-OVER}

The molecular mechanotransduction is founded on a linear propagation of steps. First, forces acting on cells and imposed by cells on the extracellular environment cause stresses and deformations that are perceived by a panel of specialized molecules termed mechanosensors (Box 1). These mechanosensors experience a force-dependent conformational alteration that modifies the biochemical functionality of the protein. Forces from the cellular environment are usually encountered primarily at the surface of the cell, where the force-generating cytoskeleton can also apply stresses as it comes into contact with various mechanical conditions. The adhesion complexes that connect cells to ambient tissues by focal adhesions and to other cells by adherens junctions have consequently been found to be key hubs in the transmission of forces (Leckband and de Rooij, 2014; Seetharaman et al., 2021; Sun et al., 2016). There are, however, a much wider spectrum of mechanosensors inherent in cells, comprising several structurally diverse families of force-sensitive ion channels (Kefauver et al., 2020) and receptors for biochemical ligands that react in a direct manner to force, such as notch (Stassen et al., 2020) and plexin (Mehta et al., 2020). In addition, forces at the cell periphery are propagated through the cytoskeleton to other cellular locations such as the nucleus (Cho et al., 2017), which also harbor mechanosensitive compounds and enable cellular reaction to external and intrinsic forces.

Mechanosensors operate using a number of common mechanisms through the force-induced conformational alterations that impact either molecular perceptual interactions or the activity of the protein. Forces directly amplify the proteinprotein interaction of mechanosensors in that they enhance the lifetime of the bond (catch bond), in contrast to the majority of protein-protein interactions in which the lifetime reduces in response to the force (slip bond) (Zhu et al., 2019). In addition, forces are able to mutilate the form of interactions through protein unfolding or unmasking, which results in either the exposure of cryptic binding sites (del Rio et al., 2009; Yonemura et al., 2010) or the breakdown of binding motifs (Ehrlicher et al., 2011). The character of the cryptic site differs among a variety of mechanosensors, and forces involved can uncover proteolytic sites (Gordon et al., 2015; Mack et al., 2017) or motifs for the purpose of posttranslational processing (Sawada et al., 2006). Multiple membrane-associated mechanosensors are adjusted in response to force-induced alterations in membrane tension, for example, through regulating the gate operation of mechanosensitive ion channels (Saotome et al., 2018). In addition, cytoskeletal forces are also capable of stabilizing certain structural conformations of mechanosensors including integrins (Li and Springer, 2018). Mechanosensors frequently constitute broader multimolecular complexes with assemblies of different mechanosensors governed by various mechanisms, prototypical instances of these being focal adhesions and adherens junctions.

Mechanosensors do not function like ordinary on-off relays, instead their reaction involves different characteristics of the forces. Forces themselves can operate on distinct sections of the cell, and can have varying magnitudes, directions, and temporal characteristics, each resulting in a specific unique reaction and varying biological results. The intrinsic mechanisms of force transmission in an individual mechanosensor, and its organization inside the cell, dictate the capacity to discriminate between these various kinds of parameters. All of which is explained in more detail in the following sections. 
What is the effect of the well-investigated plasma membrane tension? Membrane tension emerged as a crucial mechanical factor for cell locomotion, shape modification, and volume adjustment (Tao and Sun, 2015). An elevation in membrane tension is observed to augment the crawling speed of osmotically shocked Caenorhabditis elegans spermatocytes by restricting extraneous protrusions (Batchelder et al., 2011) and works as a widespread inhibitor of actin assembly, thereby aiding neutrophils to preserve polarity and restricting the pseudopod count to one (Houk et al., 2012). Membrane tension levels have also been demonstrated to direct the positioning of focal adhesions at the leading edge of mouse embryonic fibroblasts through inducing the buckling of actin filaments without the function of myosin II (Pontes et al., 2017). In addition, it can restrict actin assembly at the front edge of creeping neutrophils via a negative feedback circuit that engages phospholipase D2 (PLD2) and mammalian target of rapamycin complex 2 (mTORC2) (Diz-Muñoz et al., 2016). Each of these investigations has been conducted on stiff substrates. Thus, how would a softer substrate impact the membrane tension? In a recent investigation, membrane tension of hepatocellular carcinoma cells on soft hyaluronic acid and stiff polyacrylamide substrates has been assessed through pulling membrane tapes with optical tweezers (Mandal et al., 2019). The membrane tensions measured on soft 300 $\mathrm{Pa}$ hyaluronic acid media are somewhat equivalent to those measured on $30 \mathrm{kPa}$ polyacrylamide media, and the magnitude of the measured membrane tension does not appear to correspond to the traction forces imposed by the cells on the media. This indicates that the forces generated by cells on their extracellular matrix are not substantially modifying the membrane tension (Mandal et al., 2019).

\subsection{Viscoelasticity of Cellular Constituents}

It is not quite clear whether cytoskeletal constituents of cells possess and inherent viscoelasticity or contribute in structural terms to the global viscoelasticity of cells. There are some features of cellular components that can contribute to mechanical characteristics of cells, including viscoelasticity. In order to withstand or deform without impacting the integrity of a cell or cluster, subcellular constituents are dynamically realigned throughout cell migration. In other words, to react to external mechanical cues, cells simply need to be able to detect them. The mechanism that enables cells to sense and react to physical stress from their microenvironment comprises the sensing of mechanical cues, termed mechanosensing, and their conversion into a biochemical reaction, referred to as mechanotransduction (Charras and Yap, 2018). The mechanotransduction process enables cells to be potentially able to react to mechanical challenges through adapting not only their stiffness, but also their viscoelastic response. In order to understand the aforementioned hypothesis a brief excurse in the canonical process of mechanosensing is provided in the following.

The cellular answer to physical signals provoked by mechanical stress involves a short- or medium-term adjustment of cell shape via cytoskeletal reassortment, usually entailing post-translational modification of scaffoldings, adhesion, polarity, and contractility associated proteins (Ren et al., 2009; Moore et al., 2010; Pandya et al., 2017). Beyond this, a long-term responsiveness can also be witnessed, fueled by changes in gene expression profiles that are subsequently reflected back in cellular performance, which is far more less explored compared to the canonical process.

In adherent cells, the detection and signal transduction of mechanical cues are intricate processes, and herein are briefly concerned with a "canonical" and somewhat simplified sensing mechanism (Jansen et al., 2017; Gauthier and Roca-Cusachs, 2018; Yap et al., 2018). Among the first proposed structures participating in mechanosensing are the focal adhesion sites, where integrin adhesion receptors attach the cell tethered to the extracellular matrix (Jansen et al., 2017; Gauthier and Roca-Cusachs, 2018). This engagement elicits the assembly of a set of proteins into focal adhesions that couple this integrin receptor to the cytoskeleton (Sawada et al., 2006; Moore et al., 2010; Schiller and Fässler, 2013; Fedorchak et al., 2014; Paluch et al., 2015; Petridou et al., 2017). Finally, the force encountered at the focal adhesions is transmitted into the cytoskeleton through a protein complex composed in parts of vinculin and talin-driven force transfers (Grashoff et al., 2010; A.; Kumar et al., 2016; Sawada et al., 2006; Yao et al., 2016). The cytoskeleton then backcouples into the focal adhesion to enhance the dynamic nature of this structure through myosin II-facilitated contractility, leading to substrate distortion in cells migrating into compliant surfaces and cell distortion and migration in cells migrating over stiffer substrates. When a cell is mechanically deformed, the cytoskeleton will transduce these mechanical loads from the microenvironment via filament linkages into the nucleus to alter the expression of genes and consequently biochemical signaling (Paul et al., 2008; Fouchard et al., 2011; Paluch et al., 2015). The recognition and translation of mechanical environmental stimuli into a cellular and molecular response is not only driven by linking focal adhesions and the cytoskeleton in a bidirectional manner, mechanosensing also entails the activation of mechanosensitive ion channels, such as Piezo1 (Gudipaty et al., 2017; Hung et al., 2016; Wu et al., 2017), or mechanically reactive nuclear pores and the subsequent activation of targeted transcription factors (Elosegui-Artola et al., 2017; Stanley et al., 2017; Kirby and Lammerding, 2018; Otsuka and Ellenberg, 2018).

The mechanical response of talin is pivotal to the intricate regulatory mechanisms that manage force transfer between the actin cytoskeleton and the extracellular matrix. The principal mechanism underlying the force transmission paradigm observed between F-actin and integrins is "focal adhesion coupling (referred to as clutch)," which characterizes reciprocal interactions between relatively static, ligand-bound integrins and centripetally flowing F-actin in the vicinity of cell borders (Elosegui-Artola et al., 2018). The linkages that convey force in this environment are highly dynamic, exhibiting rapid on and off rates. The stiffness of the extracellular matrix changes the rate of loading applied through these linkages, which modifies the internal kinetics. The importance of stiffer media is that they 
increase the traction force and thereby provide stability to the adhesions (Elosegui-Artola et al., 2018), along with the force generated through the substrate stretching (Sun et al., 2016). While these effects have been inferred to the focal adhesion clutch model of dynamic force transmission (Elosegui-Artola et al., 2018), emerging evidence has questioned this modeling paradigm and revealed a more intricate mechanism. Tension analysis over talin combined with actin dynamics identified three separate mechanisms of force transfer, but only one of them is dynamic (Driscoll et al., 2020). In newly created cell adhesions close to the cell edge, force transmission occurs through fastflowing actin powered by rapid polymerization at the edge, as outlined in the clutch model. Nevertheless, when vinculin is being recruited and actin velocity drops, force transduction switches to a flow-independent transmission powered by myosin contraction. While this is coherent with the stability of vinculin-F-actin linkages under force, which are referred to as catch-bond properties (Huang et al., 2017), it is not in agreement with the short-lived linkages of the clutch model. Therefore, vinculin has more of a part to play in locking the moving actin filaments and forming stable junctions than in providing dynamic force transduction. What is important to note is that the homeostatic relationship among these mechanisms is governed by substrate rigidity, so that dynamic force transduction is actually more dominant on soft substrates, while flowindependent force transduction is prevalent on stiff substrates (Driscoll et al., 2016). A pivotal perspective involves the feedback between the forces and the sensing complex. Cells located on stiff interfaces or exposed to strain increase their adhesions and enhance the contractile force exerted by the cell, thereby altering its mechanosensation. For instance, highly contractile cells need fairly stiff supports to completely spread, whereas less contractile cells can spread fully on softer surfaces (Discher, 2005). In other words, cells can accommodate themselves to surroundings with extremely diverse mechanical characteristics.

While these are canonical mechanosensing mechanisms, the exact mechanism through which cells perceive and react to mechanical cues as they migrate as a collective is only beginning to be explored. In vivo, the fibronectin-based extracellular matrix of Xenopus, chick, or zebrafish embryos (Bajanca et al., 2019) is around $10 \mu \mathrm{m}$ thick, and recent in vitro strategies indicate that single cells can scan their substrate to a depth of around $15 \mu \mathrm{m}$ (Buxboim et al., 2010), in this respect, a mechanism similar to the aforementioned one suits ideally to the scanning of the extracellular matrix by individually migrating cells. Nevertheless, it is suggested that collectively migrating cells apply 10 times larger forces to the substrate. Therefore, the interval at which a migrating cluster of adherent cells perceives physical variations to its microenvironment is actually deeper than that recorded for individual cells (Tusan et al., 2017). But how this enhanced sensitivity is accomplished by the group of cells, or even whether this is the situation in vivo, remains to be further examined.

Since, poroelastic effects (see 8.1 for details) overlap with other mechanical responses of tissues and extracellular matrices, involving nonlinear elasticity, viscoelasticity, and viscoplasticity (Chaudhuri et al., 2020), analogous mechanisms pertain to the viscoelasticity of the cytoskeleton of cells (Mitchison et al., 2008; Hu et al., 2017; Mollaeian et al., 2018). However, there are two major dissimilarities between poroelastic effects of living cells and extracellular matrices. Firstly, the comparatively impermeable cell membrane prevents or delays poroelastic effects because of overall cell deformation, however, local contraction of the cytoskeleton can produce intracellular poroelastic actions and transient compressive gradients that are sustained for periods of biological concern (Moeendarbary et al., 2013). Secondly, the difference lies in the fact that covalent bonds between the filaments of the cytoskeleton are extremely few or absent. In this regard, moreover, motor proteins exert incidental, nonthermal forces on the filaments of the cytoskeleton (Guo et al., 2014), moving them faster than they would if driven by thermal motion only, with the net effect that the active cytoskeleton is more extensively fluidized than one lacking motors (Humphrey et al., 2002). Cellular viscoelasticity behavior can even be evident at the tissue level. For instance, rigor mortis, the stiffening and solidification of muscle after death, is due partially to the fact that the connections between actin protein filaments and myosin motor proteins in muscle fibers grow in both number and permanence as the living muscle hydrolyzes ATP, thereby allowing the actin-myosin connections to build and become dissociated quickly.

Nonlinear elasticity is also evident in cytoskeletal filament networks, such as actin, vimentin, and neurofilaments, however, the source of nonlinear elasticity in these structures may reflect a stronger input from entropic elasticity due to the semiflexible character of the filaments (Storm et al., 2005). In the following a possible role of these biological intracellular cytoskeletal, compartmental or nuclear structures may play a role in the viscoelastic characteristic of cells.

\subsection{Viscoelasticity of Focal Adhesions}

Similar to adherens junctions, focal adhesions comprise a number of mechanosensor proteins employing various mechanotransduction mechanisms. The conversion of mechanical impulses into biological events and their subsequent reaction can be broadly classified into two distinct phenomena. The first phenomenon is the active or passive reaction of cells to externally exerted forces, such as when fluid shear stresses act on the endothelium as a result of blood flow across the vasculature, or when gravity acts on joints, bone, or adipose tissue. These types of excitements provide the foundation of Wolff's law for bone remodeling, the connection between modified blood flow and the onset of atherosclerosis, or the stress of expanding cancers (Yusko and Asbury, 2014). The second phenomenon is the cell and tissue reactions resulting from the forces produced directly by the cell, which are resisted due to the viscoelastic or active characteristics of the extracellular matrix or the ambient cells.

The transduction of biochemical cues frequently involves allosteric alterations in protein conformation or phosphorus regulation. Fluctuations in phosphorylation levels can directly affect the activity of a binding moiety or an active site within an 
enzyme, and they can cause conformational rearrangements in a manner akin to allosteric effects. Since mechanical stresses on a protein have also been found to modify its conformation, evolution efforts not surprisingly have yielded molecules that leverage force-dependent conformational modifications to convey and transduce mechanical inputs (Ingber, 1997). The model describing the structural basis for mechanotransduction is termed tensegrity model.

Cellular reactions to mechanical stimuli including flow, stiffness or viscoelasticity of the extracellular matrix, and tissue extension are related to the magnitude of the forces connected to these stimuli. The range of force magnitudes perceived by the cells and the susceptibility of the various mechanosensors in this range dictate how the cells react to the mechanical stimuli. Even though the molecular underlying mechanisms of susceptibility to force magnitudes have not been fully elucidated, multiple mechanisms whereby cells can derive this kind of information have been identified. A molecular concept for sensitivity to force magnitudes is that mechanosensors exhibit a critical threshold force for activation, such as the force necessary to uncover cryptic binding sites or the force regime where capture bonds are established. Moreover, this sensitivity can be finely tailored through the presence of stable intermediate modes for the force-induced conformations of specific mechanosensors. For example, single-molecule force spectroscopy of catch bonds identified three main modes (weakly, intermediately, and strongly bound) at a variety of force levels for fibronectinintegrin (Kong et al., 2009), vinculin-F-actin (Huang et al., 2017), and VWF-GPIb (Ju et al., 2013).

RhoA has been determined to fulfill a function in mechanotransduction that depends on the tension exerted on fibronectin-coated beads attached to the surface of the cell membrane (Matthews et al., 2006) and additionally tension on integrins has been seen to activate RhoA (X.-H. Zhao et al., 2007). Activation involves either activation of a GEF or engagement of a GAP. GEF-H1 and LARG have been revealed to be active in reaction to tension on integrins (Guilluy et al., 2011). Examination of the signaling pathways upstream of these GEFs indicated that LARG is activated through phosphorylation of the Src family kinase Fyn, whereas GEF-H1 is activated through the MEK/ERK pathway after focal adhesion kinase (FAK) activation (Guilluy et al., 2014).

Activation of GEFs that occurs in reaction to mechanical force on fibronectin-coated beads is a downstream reaction to stressinduced activation of kinases, specifically members of the Src kinase family and/or FAK. The activation of FAK takes place after integrin entanglement and establishment of focal adhesions (Burridge et al., 1992). Inhibition of tension on integrins by hampering myosin activity using blebbistatin diminishes activation of FAK (Pasapera et al., 2010). It has been demonstrated that tension on integrins facilitates integrin binding to the synergy site of fibronectin in addition to the RGD binding site in a manner that stimulates activation of FAK (Friedland et al., 2009). In agreement with this result, others have shown that tension on fibronectin uncovers the synergy binding site causing the $\alpha 5 \beta 1$ integrin to attach to it and also to the RGD binding site (Seong et al., 2013). It can be anticipated that FAK is clustered as a consequence of full integrin-fibronectin binding and that clustered FAK is transphosphorylated, thereby causing FAK activation. Consequently, tension can be applied to FAK to free it from the auto-inhibited conformational state (Zhou et al., 2015). In line with this, other focal adhesion proteins, including talin, are stretched due to mechanical tension applied to integrin-based adhesions (del Rio et al., 2009). The question is whether these other deformations proceed in concert with elongation and activation of proteins, including talin, vinculin, Src and FAK, or whether they follow one after the other.

Focal adhesions can be altered based on the turnover of focal adhesion proteins and the stretching of focal adhesion proteins that act as mechanosensors. There are several mechanisms through which focal adhesion proteins can be altered by a stretching force: the cryptic binding site, such as talin, and the cryptic phosphorylation motif, such as p130Cas. When talin connects integrins and actin, it transfers both cell-generated contractile forces and forces originating from externally applied loads across these constituents. Forces sampled through talin span from just a few to over 11 piconewtons (Austen et al., 2015; Driscoll et al., 2020; Kumar et al., 2016). Talin reactions to forces share four characteristics with key implications. Firstly, forces tend to keep the elongated conformation of talin stable (Khan and Goult, 2019) because the head and tail are kept separated through tension, thereby constraining the autoinhibition conveyed through head-tail interactions. Secondly, the coupling of talin with actin and with integrin displays catch-bond characteristics, which means that the binding strength increased in response to moderate forces (Owen et al., 2020) that additionally stabilizes the activated, committed conformation. Third, the force unwrinkles the helical bundles of the talin rod domain; which simultaneously breaks the bonds of proteins tethering the folded state and uncovers binding sites for others. Fourthly, the unfolding of the talin rod domain presents hysteresis, which means that the force necessary for unfolding is stronger than the force needed to refold it. When a rod domain unfolds due to a force of $10 \mathrm{pN}$, it fails to refold instantaneously when the force is less than $10 \mathrm{pN}$. Refolding demands much lower tension of approximately $1-3 \mathrm{pN}$ (Yao et al., 2016). In this way, the basic physiological forces of around $5 \mathrm{pN}$ on talin inside focal adhesions (Kumar et al., 2016) tend to keep the patterns of folded and unfolded talin rod domains stable (Yao et al., 2016). Consequently, these characteristics confers a mechanical memory to the talin (Goult, 2021).

The structure-function analysis of talin lead to a model for mechanotransduction. High affinity sites for vinculin had been charted on the talin rod, however succeeding structures of the respective domains exhibited that these sites remained masked (Papagrigoriou et al., 2004). This led to the general idea that mechanical unfolding of the talin domain is necessary for vinculin binding, which has later been corroborated using single-molecule biophysics (del Rio et al., 2009; Yao et al., 2015). This original scheme has been further extended when it was found that the Rap1 effector RIAM attaches to the folded 
R3 domain. In this case, the force dislodges RIAM and thereby enlists vinculin, which constitutes a smart mechanical toggle that alters the affinity for the two ligands (Goult et al., 2013; Lee et al., 2013; Vigouroux et al., 2020; Wang et al., 2019). The existence of 13 such domains across the Talin rod, which unfold at distinct forces, is fascinating and raises options for highly intricate force sensitivity, including time-dependent effects (Yao et al., 2016).

Force-independent cross-talk between talin and vinculin has also been noted (Atherton et al., 2020; Austen et al., 2015; Han et al., 2021; Kelley et al., 2020), even though such cross-talk involves the gradual elimination of autoinhibition of the two proteins. This fits with the hypothesis that these proteins need to interact initially in a non-mechanical fashion to establish the bonds for force transfer. When force is exerted, unfolding of the talin helix bundle domains uncovers vinculin binding sites, which bind and further act to stabilize the active conformations of talin and vinculin (Wang et al., 2021; Yao et al., 2015). Therefore, the force is also a stabilizer for the open state of talin and vinculin, which is retained even after a severe decrease in stress (Yao et al., 2015). The binding of vinculin also provides linkages to F-actin, which may increase the force on talin and promote greater force transfer (Kumar et al., 2016). Higher tension levels subsequently further enhance the forces on neighboring areas. Thus, there are several molecular pathways that, once talin is open and in tension, it sustains talin domains in an open conformation of high tension. Oppositely, ligands attached to folded talin-helix bundles act to stabilize this conformation and enhance the force necessary for opening, which depends on the expression and affinity of the ligands. Thereby, the closed configurations are made subject to a positive feedback loop. These mechanisms stabilizing open or closed conditions constitute a type of molecular memory. Consequently, these mechanisms significantly prolong the open and closed condition lifetimes for each molecule (Khan and Goult, 2019; Wang et al., 2019; Wang et al., 2021), an essential type of mechanosensitivity.

What role plays the interaction of DLC1 and talin? The tumor suppressor deleted in liver cancer 1 (DLC1) seems to be important due to its recruitment to focal adhesions (Kawai et al., 2004). In focal adhesion, DLC1 can interact with the mechanosensitive protein focal adhesion protein talin. Specifically, DLC1 is found to bind to the R8 domain of talin, thereby DLC1 is clearly localized in focal adhesions and activated, whereby it causes a reduction in the active RhoA level. Nevertheless, when tension is enough to stretch talin and open the R8 domain, DLC1 is liberated in a conformationally constrained manner and accounts for enhanced RhoA activity (Haining et al., 2018). The liberation and subsequent deactivation of DLC1 from stretched talin suggests an additional pathway by which mechanical tension imposed on integrin adhesions is capable of augmenting RhoA activity. Therefore, a potential negative feedback pathway may also be responsible, which could be relevant in restricting the focal adhesion size. It recognized that mechanical tension encourages the growth of focal adhesions via a RhoA-dependent pathway (Riveline et al., 2001). Tension at focal adhesions stretches their constituents such as talin, thereby enlisting additional binding partners (del
Rio et al., 2009). Large adhesions, nonetheless, have been found to produce lower traction than small adhesions (Beningo et al., 2000), and FRET-based stress sensors have demonstrated that lower tension is transferred to constituents within large focal adhesions compared to small adhesions (Austen et al., 2015; Grashoff et al., 2010; Kumar et al., 2016). A negative feedback circuit needs to be in place to avoid additional growth of the focal adhesions as a response to the growing tension.

\subsection{Cytoskeletal Viscoelasticity}

The cytoskeleton functions not merely as an integral regulator of molecular circuitry, but also as a mesoscale mechanosensor with its inherent level of regulation and dynamics. Specifically, the cytoskeleton has a critical involvement in the majority of suggested mechanisms for conveying the perception and transmission of mechanical signals from the microenvironment, and its constituents experience large alterations during cellular deformation in response to mechanical stress (Wen and Janmey, 2011). The basic elements of the cytoskeleton are biological polymers known as intermediate filaments, actin filaments and microtubules (Fletcher and Mullins, 2010; Stamenović and Wang, 2000). An equilibrium of the polymerization/depolymerization fraction and the extent of crosslinking of these structures governs whether a cell distorts or withstands deformation when exposed to mechanical stress (Figure 2). The degree of build-up and breakdown of these elements is regulated by a number of molecules. For instance, actin nucleation factors induce and elongate polymer filaments, capping factors cease the growth of filaments, and there are depolymerizing and detaching factors that break down these filaments (Stamenović and Wang, 2000; Wear et al., 2000; Carlsson, 2010; Reymann et al., 2012; Salbreux et al., 2012; Dang et al., 2013; Krause and Gautreau, 2014; Veltman, 2014).

The mechanical performance and characteristics of these three filaments have been characterized in various systems. Microtubules emerged as the more rigid part of the cytoskeleton, with a less rigid meshwork of actin filaments and a more soft meshwork of intermediate filaments (Fletcher and Mullins, 2010; Hawkins et al., 2010; Stricker et al., 2010; Wen and Janmey, 2011; Salbreux et al., 2012; Lopez and Valentine, 2015; Charrier and Janmey, 2016). Mechanical stress on the cytoskeletal reticulum has also been assessed in epithelial cells through flow analysis, and mechanisms monitoring the stiffening of the cytoskeleton have been put forward (Helmke et al., 2003; Žagar et al., 2015). Apart from the polymerization and passive mechanical characteristics of its constituents specifying the mechanical condition of the cytoskeleton, it has also been theorized that crosslinking factors have a part to play in defining the architecture of the cytoskeleton's filaments and, as a result, their elastic condition (Brighenti and Vernerey, 2017; Harris et al., 2012; Kirschner, 1986; Lieleg et al., 2009). The activity of molecular motors for instance myosin II in conjunction with cross-links is also connected to the viscoelastic characteristics of the cytoskeleton (Paul et al., 2008; Murrell et al., 2015). The effective coordination between filaments, motors and cross-links is mechanically excited. By way 


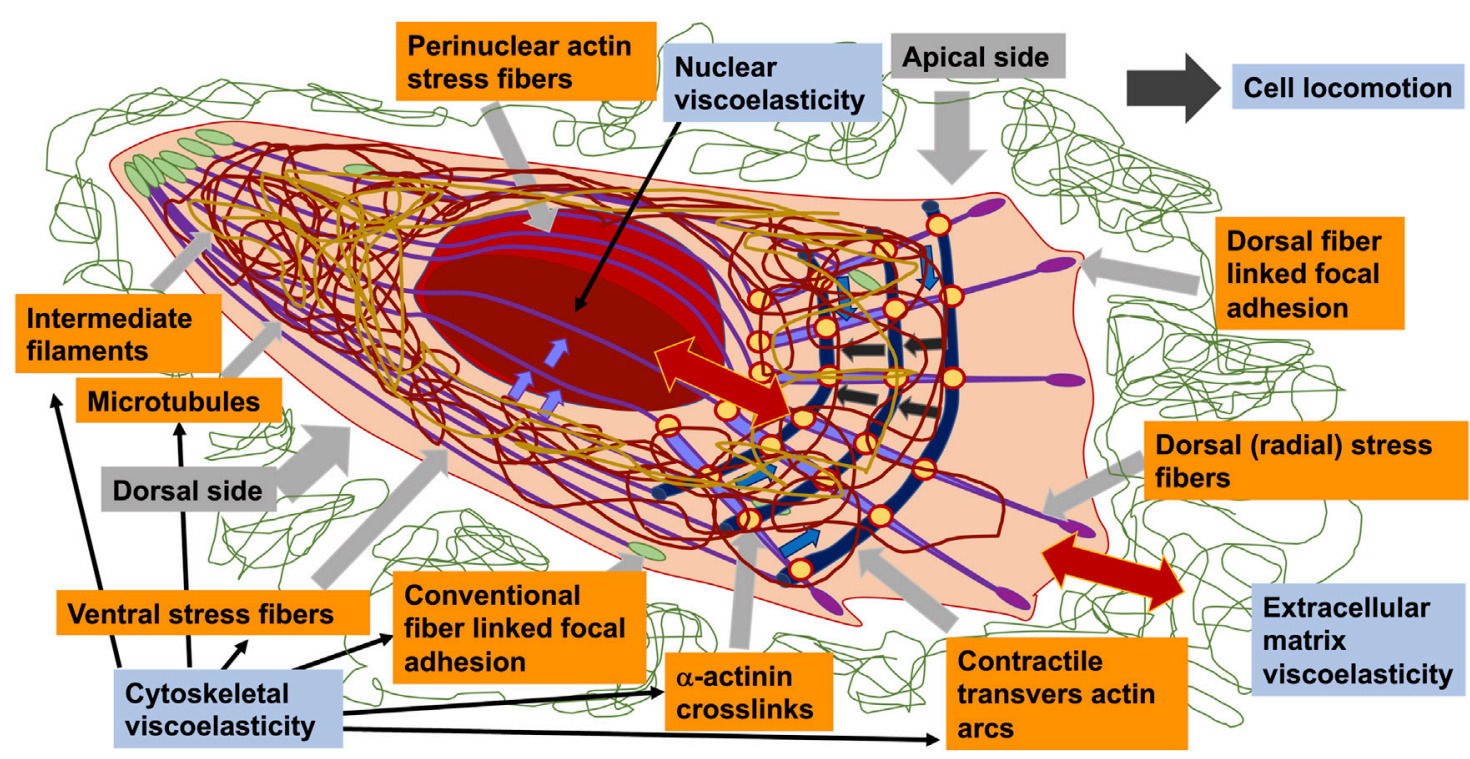

FIGURE 2 | Possible viscoelastic features of cellular constituents, such as nucleus, cytoskeleton that interact through mechanotransduction processes with the viscoelastic characteristics of their natural environments, such as extracellular matrix scaffolds of connective tissues.

of illustration, the engagement of myosin with actin fibers proceeds in a force-dependent fashion, the same as the contractile reaction of actomyosin to extracellular stiffness, as evidenced through cell elongation and real-time imaging (Mitrossilis et al., 2010; Fouchard et al., 2011). Actin assembly assays reveal that force-generating actin webs accommodate to external mechanical forces and that force reconnection between intracellular and extracellular forces improves the tightness and mechanical efficacy of newly ramified actin webs (Bieling et al., 2016).

What about the viscoelastic properties of cytoskeletal filaments? Actin networks cross-linked by filamin have been found to display dramatic macroscopic stress-hardening response. By imposing a prestress, the nonlinear stiffness is adjustable over multiple orders of magnitude, whereas the linear elasticity of the reticulation stays at a moderate level (Gardel et al., 2004). In contrast, for instance, to cross-linked actin/heavy meromyosin webs, in which the inclusion of heavy meromyosin leads to a very marked rise in linear reticular elasticity (Tharmann et al., 2007). In filamentous actin/filamin reticulations, the nonlinear response may be accounted for by the high flexibility of the discrete filamin molecules (Gardel et al., 2004; Gardel et al., 2006a). Moreover, in actin/filamin bundle reticulations, the branched and fused reticulation microstructure (Schmoller et al., 2008) could also be considered to account for the striking nonlinear viscoelastic response of the reticulation.

Contractile forces are extensively produced through the interaction of myosin II with actin filaments. GTP-bound RhoA upregulates myosin II activity through stimulation of Rho kinase (ROCK), that in turn increases phosphorylation of regulatory myosin light chain. This occurs in two ways, through direct phosphorylation of regulatory myosin light chain (Amano et al., 1996) and through the phosphorylation and consecutive blocking of the myosin light chain phosphatase (MYPT) (Kimura et al., 1996). Phosphorylation of myosin light chain increases the assemblage of myosin II into filaments and boosts its ATPase activity, which enhances the contractile force applied from myosin II to actin filaments. When myosin is arranged as filaments myosin, it acts as a prominent bundling protein for filamentous actin. Besides, ROCK phosphorylates and activates LIM kinase so that it can phosphorylate and restrain the actinseparating protein cofilin (Maekawa et al., 1999). Thereby, the actin filament stability is improved. RhoA stimulates also the continued assembly of actin filaments via its $\mathrm{mDia}$ effector, which represents an actin nucleating protein belonging to the formin family (Watanabe et al., 1999). Consequently, the RhoA signal transduction pathway is extensively accountable for a large part of intracellular force production in cells (Chrzanowska-Wodnicka and Burridge, 1996).

Cytoskeletal filament networks show viscoelastic behavior, since the disruption of the binding motif of filamin A that acts as an actin filament cross-linking protein. Additionally, the cryptic phosphorylation motif in $\beta$-catenin can be exposed by forces and thereby lead to viscoelastic behavior of the cytoskeleton. Moreover, the generation of catch bonds in $\alpha$-catenin due to external stimulation can also cause elevated viscoelastic characteristics of cytoskeletal filament networks. Thus, this implies that coupling back between external and internal mechanical force may assist migrating cells to tailor their viscoelastic characteristics and migrate in physically confined microenvironments.

\subsection{Nuclear Viscoelasticity}

When a solid tumor expands due to cell proliferation, it generally gets denser, and the cancer cells are subject to a harsher physical environment. In part this is attributable to the deposition of more 
extracellular matrix, as well as cell proliferation resulting in tighter packed cells within the disposable tissue volume, and enhanced contractility of stromal cells (Paszek and Weaver, 2004; Paszek et al., 2005; Jaalouk and Lammerding, 2009; Yu et al., 2011). Cancer cells that metastasize are subjected to multiple forces, whether by invasion of individual cells or by collective cell migration (Friedl and Wolf, 2010). Squeezing across tight gaps in the extracellular matrix subjects cancer cells to substantial compressive and tensile forces, which can be adequate to induce transient break down of cell nuclei and liberation of chromatin, resulting in DNA damage (Denais et al., 2016; Raab et al., 2016). In addition to chemical cues that govern cell performance, mechanical forces can have a critical regulatory effect on cellular function. In response to various forms of mechanical forces applied to the cell membrane surface, multiple signaling cascades are excited. These include stretch-activated ion channels to activation of kinase cascades and Rho GTPases (Orr et al., 2006; Wang et al., 2009). In the end, multiple of such signaling pathways impact the transcription of genes.

\subsubsection{Role of Forces on Gene Expression}

Small molecules and genetic interferences are frequently used to affect regulatory pathways, for instance pathways regulating calcium content or Rho GTPases (Loirand and Pacaud, 2014). The commonly used biochemical and genetic modifications cannot ensure the tight spatiotemporal monitoring of cell contractility. This complicates their applicability to discover how local elevation or attenuation of contractility might cause cellular or multicellular shape alterations. Optogenetics can be used to achieve reversible disturbance of intracellular biochemistry with subcellular resolution by expressing genetically engineered light-sensitive proteins. Optogenetics is a technique that has been utilized effectively to regulate the activity of ion channels, phospholipids, actin polymerization components and RhoGTPases (Bugaj et al., 2013; Deisseroth, 2011; Guglielmi et al., 2015; Idevall-Hagren et al., 2012; Levskaya et al., 2009; Rao et al., 2013; Valon et al., 2015; Wagner and Glotzer, 2016; Wu et al., 2009). Two optogenetic instruments that rely on monitoring the activity of endogenous RhoA to either upor down-regulate the contractility of cells. These instruments elicit fast, spatial, and reversible alterations in traction forces, cellcell forces, and the compaction of tissues. Thus, in addition, the changes in cellular forces are accompanied with translocation of the Yes1-associated transcriptional regulator (YAP), which is synonymously referred to as YAP1, indicating that optogenetics can be employed to govern mechanotransduction signaling pathways (Valon et al., 2017).

A candidate for regulating RhoA activity is the DHPH domain of ARHGEF11 (Rossman et al., 2005; Zheng et al., 2009), which fuses with CRY2-mCherry to form ARHGEF11(DHPH)-CRY2mCherry, designated optoGEF-RhoA. To precisely govern the localization of this protein, two specific types of CIBN have been generated, one of which is directed to the plasma membrane (CIBN-GFP-CAAX) and one to the mitochondrial membrane (mito-CIBN-GFP). Through the application of an infrared RhoA biosensor composed of the rhotekin-binding domain (RBD) merged with infrared fluorescent protein (iRFP), it has been discovered that local enrollment of optoGEF-RhoA at the cell membrane corresponds to elevated activity of RhoA. Therefore, the optogenetic approach enables fast and reversible targeting of the catalytic domain of ARHGEF11 to the plasma membrane or mitochondria, which leads to supervised RhoA activity.

Additionally, it has been examined whether RhoA activation is accompanied with alterations in cell contractility after relocation of optoGEF-RhoA to the cell membrane or to the mitochondria. Hence, Traction Force Microscopy (Trepat et al., 2009) has been adopted to quantify the forces exerted by the cells on the subjacent soft collagen I-coated substrate $(12 \mathrm{kPa}$, polyacrylamide) encountered throughout optogenetic activation and deactivation. To investigate the function of optoGEF-RhoA translocation toward the cell membrane, a MDCK cell line is genetically engineered that stably coexpresses optoGEF-RhoA and CIBN-GFP-CAAX. In order to determine the tension within a cell and among two neighboring cells, Monolayer Stress Microscopy has been utilized (Tambe et al., 2013). Similar to the traction forces, the cell tension grew in the exposed areas and stayed the same in the non-exposed areas. The growth in tensile force and tension has been maintained for a minimum of $40 \mathrm{~min}$ and, crucially, has been completely reversible. These findings demonstrate that mastering the subcellular localization of the catalytic domain of ARHGEF11 provides spatiotemporal inroads into the regulation of signal transduction and, consequently, cell contractility. The optogenetic enhancement of contractility has been coincident with the development of actin stress fibers (Valon et al., 2017). Reciprocally, cell relaxation has been associated with the vanishing of basal stress fibers. The pattern of spatial distribution of focal adhesions in the presence of alterations in contractility has been evaluated through co-transfection of optogenetic constructs and vinculin-iRFP. An augmentation of contractility failed to produce systematic modifications in the size or dispersion of focal adhesions. Optogenetic contraction and relaxation are hypothesozed to coincide with structural alterations in stress-generating and stress-sensing components of the cell.

To examine this hypothesis, the transcriptional regulator YAP has been selected, which has been reported to be translocated from the cytoplasm to the nucleus due to sustained elevated traction forces or increased substrate stiffness (Dupont et al., 2011; Elosegui-Artola et al., 2016). Therefore, it has been explored whether a sustained raise in cell contraction causes the translocation of YAP. For this purpose, MDCK cells are cotransfected with optoGEF-RhoA, iRFP-YAP, and CIBNGFP-CAAX and exposed to pulsed illumination. Throughout this process, the intensity of iRFP-YAP within the nucleus has been assessed. The optogenetic enhancement of contractile forces has been accompanied with an enhancement of YAP in the nucleus. Consequently, optogenetic contraction and relaxation of cell contractility have antagonistic impacts on YAP translocation in the nucleus, suggesting that optogenetics can be employed to properly modify mechanosensitive transduction pathways and to interrogate potential transcriptional alterations. YAP is a major actor in the coordination of tissue growth, 
homeostasis, and cancer formation. It is controlled through two independent pathways. The first, entirely biochemical, incorporates the Hippo signaling pathway, while the second directly engages mechanosensitive signaling pathways that translate mechanical cues into biochemical cues.

\subsubsection{Role of Nuclear Characteristics on Cell Migration}

Intriguingly, not merely the cytoskeleton and its elements account for the viscoelasticity of cells (Fischer et al., 2020). Apart from the cytoskeleton, other compartments of the cell, such as the biggest organelle of the cell, the cell nucleus contributes to the mechano-phenotype of the overall cell. It has also been revealed that the nucleus may be a constraining agent on the degree to what a cell would deform to pass through restricted cavities (Friedl et al., 2011; Calero-Cuenca et al., 2018; Fischer et al., 2020). The nucleus is enclosed by a nuclear membrane, beneath which is a dense meshwork of proteins referred to as the nuclear lamina, the principal constituent of which comprises the nuclear intermediate filaments, primarily made up of proteins referred to as lamins (Davidson and Lammerding, 2014; Fedorchak et al., 2014). Lamins are a vital part of nuclear-cytoskeletal interactions and mutations on these molecules affect the biochemical answer of cells to a mechanical impulse (Lammerding et al., 2004, Lammerding et al., 2005; Cupesi et al., 2010; Wolf et al., 2013; Cho et al., 2017). Lamin seems to directly function in the matrix-driven differentiation of tissues. High matrix stiffness values due to accumulation of collagen fibers cause high levels of lamin A and lead to the differentiation to bone, whereas low stiffness levels result in lower amounts of lamin and cause the differentiation toward fat tissue (Swift et al., 2013). Moreover, the cell nucleus serves a critical function in the progression of cell migration through constrained volumes, as it provides a mechanical barrier that impairs the amount of cell deformation that a migrating cell might encounter. Strictly stated, the nuclear lamina acts as a constraining element for the deformability of the nuclear core (Wolf et al., 2013; Calero-Cuenca et al., 2018). Whereas high levels of lamins provide a roadblock to deformation, lower levels permit a more flexible nucleus, but ultra-low levels of lamins restrict the survival of the cell (Lammerding et al., 2004; Cupesi et al., 2010; Ho and Lammerding, 2012). Among the mechanisms governing the density of the core lamina and the viscoelastic characteristics of the nucleus has recently been delineated through an elegant hybridization of microfabrication and quantitative biology techniques. Thereby, fast build-up of the actin nucleator Arp2/3 at the nuclear membrane of dendritic cells corresponds to instability of the nuclear lamina, which permits cells to deform their nuclei (Thiam et al., 2016), something that could be pertinent in vivo, where cells have to modify their viscoelasticity as they migrate through restricted gaps.

Cell transitions, such as EMT, lead to cytoskeletal alterations of cells (Yilmaz and Christofori, 2009) that can affect their extracellular matrix environment, since both the cytoskeleton of cells and the extracellular matrix are intrinsically coupled (Leggett et al., 2021). Consequently, cells are able to perturb the structure of the local matrix through deformation and remodeling in order to foster their migration and invasion.

\subsubsection{Viscoelastic Model of the Nucleus}

The nuclear rheology relies on the time and length scales of the forced deformations that are determined by a variety of techniques ranging from substrate stretching (Lammerding et al., 2006), indentation (Schäpe et al., 2009; Krause et al., 2013), microneedle-based micromanipulation (Shimamoto et al., 2017; Stephens et al., 2019) and micropipette aspiration (Dahl et al., 2004; Guilak et al., 2021). This intricate viscoelastic reaction mirrors the wide range of both weak and strong interactions between lamins and chromatin (Dahl et al., 2005), which located at the nuclear envelope (Gruenbaum et al., 2005; Solovei et al., 2013) as well as in the nucleoplasm, and contributes to the stabilization of condensed chromatin regions inside laminassociated domains (Guelen et al., 2008). Micromanipulation analysis yielded a common length scale of about $3 \mu \mathrm{m}$ for the deformations, below which the elastic resistance of the nucleus is determined by chromatin, while the resistance to large deformations is governed directly through lamin A/C (Dahl et al., 2004; Stephens et al., 2019). In a similar way to isolated chromatin fibers, which stretch elastically (Cui and Bustamante, 2000), the rheological characteristics of nuclear chromatin fluctuate between predominantly elastic and predominantly viscous and are regulated via the nuclear envelope ligaments (Schreiner et al., 2015). The lamin A/C content varies more than the lamin B1 content between the cell types domiciled in the tissue to match the microelasticity of the tissue, thereby matching the stiffness of the nucleus with the extracellular stiffness (Swift et al., 2013). Concordant with the thin and low-density network of soft lamin filaments (Turgay et al., 2017), the lamina on its own, including both the A- and B-type lamina networks, imparts low mechanical strength on the nucleus (Panorchan et al., 2004; Banigan et al., 2017).

Micropipette aspiration can be used to determine the mechanical reaction of nuclei in intact cells across physiological length scales and loads. To assess the viscoelastic contributions of lamin A by itself, lamin-B1 by itself, lamin A and -B1 together, and lamin A phosphorylation levels, stable cultures of lamin knockout and lamin rescue mouse embryonic fibroblasts have been made. The mechanical function of chromatin has been analyzed with the aid of a compound pharmaceutical inhibitor of chromatin deacetylation, which reversibly causes chromatin decondensation. The integrated mechanical functions of lamins and chromatin have been dissected through RNA and protein profiling and transmission electron microscopy. A minimal linear viscoelastic model has been developed that examines the interrelated mechanical inputs of lamins and chromatin. A time scale is apparent of about $2 \mathrm{~s}$ that discriminates between two time-regimes that reveal distinct mechanical reactions of the nucleus to imposed stress. At short time periods, the nucleus expands elastically and effectively softens at long time periods. Effective nuclear stiffness is governed through lamin A and B1 and condensed chromatin. In cells expressing lamin $\mathrm{A}$, decondensation of chromatin results in nuclear stiffening, possibly due to newly 
formed interactions with the nuclear envelope. In steady condition, the nucleus deforms viscously and is governed entirely by lamin A. The universality of the viscoelastic fourelement model is not only shown for lamin expression patterns, phosphorylation levels, and chromatin condensation levels, but is also confirmed for nuclei of embryonic and pluripotent stem cells, whose lamin A and B1 levels and chromatin compaction are far reduced in comparison with fibroblastic cells. In the following the three-element models are presented and discussed. Creep test observations reveal complex viscoelastic reactions to applied loading that are a function the expression and phosphorylation of lamin, and the decondensation of chromatin. Cell nuclei across at each conditions display the following features. In the elastic reaction, the nucleus expands instantaneously at the moment the stress is imposed, similar to a spring. In viscoelastic stretching, the nucleus is viscoelastically sucked into the micropipette over a typical time scale. For viscous deformation, nuclear creep converges to a steady rate. This specific mechanical reaction has been seen at both low and high strain and is commonly used by mouse embryonic stem cells and induced pluripotent stem cells, although the organization of nuclear laminae and chromatin is quite dissimilar to that of mouse embryonic fibroblasts (Melcer et al., 2012; Schlesinger and Meshorer, 2019).

All pertinent three-element viscoelastic models consistently could not accurately account for the principal characteristics of the nuclear reaction to imposed forces under all constraints within the measured time interval. The minimum linear viscoelastic model that correctly reproduced the nuclear deformations under all constraints has been the four-element Burger model. Specifically, the Maxwell and Kelvin illustrations of the Standard Linear Solid model are in fact the only two three-element models that account for instantaneous deformation. Therefore, their usefulness in modeling the aspiration-creep compliance curves of micropipettes is analyzed and compared with Burgers model. The Burgers model yielded the highest R-squared values for quality of fit, not just because it is composed of four items. It also accounts for the viscous deformation of the nuclei at stationary state, in contrast to the standard Linear Solid models. Actually, both standard linear solid models exhibited weaker fits for nuclei expressing lamin B while lacking lamin A, due to their longterm low viscosity deformation characterized by high steepness while maintaining stiffness.

The response of the Burgers material enables the nucleus to accommodate the applied impacts through elastic elongation and relieve the sustained stresses through viscoelastic deformation to avoid cracking and breaking of the genome and lamella (Dahl et al., 2004). Natural and synthetic biomaterials consisting of cross-linked filaments frequently demonstrate nonlinear mechanics and, in particular, stiffening (Kang et al., 2009). To investigate nuclear performance under various loading levels, micropipette aspiration of WT and TKO cells is conducted with low (below $0.8 \mathrm{kPa}$ ) and high (over $1.6 \mathrm{kPa}$ ) aspiration pressure. At both pressure levels a Burgers reaction has been seen. Nuclear compliance is reduced with raising load. WT nuclei can be matched using higher stiffness terms and viscous terms, while the response time $\tau$ stays unchanged. The relationship between WT and TKO remained the same between low and high load conditions. Stiffness is defined as being governed by both lamin A and lamin B1, while viscosity is governed predominantly through lamin A (Wintner et al., 2020). Consistent with this, the viscoelasticity of chromatin has been determined using dynamic measurements in living cells, that is also viscoelastic modeled after the Langevin equation and an approximation of the harmonic potential encountered at each chromatin location (Vivante et al., 2020).

The viscoelastic characteristics of chromatin have also been measured by performing long-range coherence analysis of histone dynamics inside HeLa cells (Zidovska et al., 2013). The coherence has been found in the order of micrometers, which is larger than the typical dimension of a chromosome territory, and it has also been accounted for by the elastic characteristics of chromatin. When chromatin exhibits considerable elasticity, it can transfer local forces over large spans, even extending beyond the size of a chromosome area, which could lead to coherent movements of broad areas of chromatin. This has also been investigated theoretically, demonstrating the time-space crosstalk of chromatin loci (Lampo et al., 2016). Therefore, a coarse-grain model of chromatin has been proposed, where every two monomer segments are linked through springs, along with a hydrodynamic resistance in the nucleoplasm. This underlines the significance of the elasticity of the polymer in conjunction with the viscoelastic characteristics of the nucleoplasm.

\section{COUPLING BETWEEN VISCOELASTICITY AND CELLULAR MOTILITY}

Mobility, growth, and homeostasis are regulated by viscoelastic or material properties of cells and tissues (Huber et al., 2013; Barriga and Mayor, 2019; Burla et al., 2019; Petridou and Heisenberg, 2019; Chaudhuri et al., 2020). Viscoelasticity permits living systems to maintain a fundamental architecture because of their solid-like features, while dynamically rearranging themselves in various shapes and configurations based on their viscous properties (Lecuit et al., 2011; Pegoraro et al., 2017; Petridou and Heisenberg, 2019) that is linked to the migratory capacity of cells (Petridou and Heisenberg, 2019). Cellular viscoelasticity affects several single-cell properties including shape, division, and motility and is mainly governed by the physical characteristics of the underlying cytoskeletal meshes (Pegoraro et al., 2017). Tissue-level viscoelasticity has been found to matter in collective morphogenetic events involving tissue folding, spreading, wound healing, and migration, and is mostly dictated by the interaction of cell-cell and/or cellextracellular space relationships (Calvo et al., 2013; Barriga and Mayor, 2019; Petridou and Heisenberg, 2019).

Resembling the viscoelasticity of nonliving materials, the viscoelasticity of cells and tissues is a characteristic that emerges from the underlying architecture and is delineated by how macromolecules and cells interface (Bi et al., 2015, Bi et al., 
2016; Broedersz and MacKintosh, 2014; Farhadifar et al., 2007; Huber et al., 2013; Pegoraro et al., 2017; Petridou et al., 2021; Pritchard et al., 2014). In nonliving materials, various theoretical approaches have long been employed to connect microscopic texture to macroscopic viscoelastic characteristics (Box 1), demonstrating that viscoelasticity can act as an emergent characteristic (Alvarado et al., 2017; Bi et al., 2015; Bi et al., 2016; Gardel et al., 2004; Kim et al., 2017; Petridou et al., 2021). Similarly, temperature, for instance, arises nontrivially from microscopic particle movement in statistical mechanics. How macroscopic viscoelasticity can be modeled through the interactions of the microscopic components of living cells and tissues is an outstanding question at the border between physics and molecular and cellular biology.

An interesting empirical finding is that the material characteristics of the microscale constituents of cell and tissue viscoelasticity, for instance, the components of the cytoskeleton and the cells, respectively, generally do not correspond to the macroscale material characteristics of cells and tissues (Alvarado et al., 2017; Broedersz and MacKintosh, 2014; Petridou et al., 2021). Macroscopic viscoelasticity often displays nonlinear variations that are not evident at the microscopic scale. However, experimental evidence has been provided for such examples like the stiffening reaction of the cytoskeletal reticulum (Gardel et al., 2004; Gardel et al., 2006b; Pritchard et al., 2014), phase transitions charges of cell motions (Mongera et al., 2018) or sudden alterations in tissue viscosity (Petridou et al., 2019) (Box 1). In the aforementioned contexts, the mechanical resistance of the individual microscopic constituents to forces is not sufficient to account for the macroscopic viscoelastic variations, so instead it is important to examine the overall interaction scheme among the constituents. For example, experimental measurements of the viscoelasticity of cells and tissues typically involve applying an external force to the system, for instance employing a micropipette or a magnetic field (D'Angelo et al., 2019; Serwane et al., 2017), for a specific time period in which the deformation of the cell or tissue system is overseen. Parameters like elastic modulus, viscosity and yield stress (Box 1) can be derived from these experiments (Forgacs et al., 1998; Bonn et al., 2017).

At the nuclear scale, a link between the mechanical characteristics of the nucleus and cancer metastasis has recently begun to evolve. The nucleus, the largest cellular organelle, is quite stiff in normal cells, but once it becomes less elastic, it can be more readily reshaped and ceases to act as an interfering barrier when the cell undergoes metastasis across narrow capillaries (Contu et al., 2018; Denais and Lammerding, 2014).

\subsection{Viscoelasticity Drives Cell Migration and Invasion of Single Cells}

The capacity of eukaryotic cells to withstand deformation, conduct intracellular trafficking, and alter their shape during locomotion relies on the cytoskeleton, an interconnected reticulation of filamentous polymers and regulatory proteins.
Some recent efforts have indicated that both internal and external physical forces can function through the cytoskeleton to affect local mechanical characteristics and cell performance. The mechanics of eukaryotic cells are dominated by the cytoskeleton, a composite polymer network that embraces the whole cell and affords the cell both the stability necessary to resist external forces and the versatility to wriggle through tissue spaces (Fletcher and Mullins, 2010). The mechanical characteristics of single cells seem to determine their mode of migration, the directionality of the movement, movement dynamics and migratory capacity including invasion path length. The mechanical properties of cells additionally contribute to their force exertion on their extracellular matrix environment, such as causing the alignment of extracellular matrix fibrils, extracellular matrix degradation by release of enzymes, such as matrix metalloproteinases, or cross-linking of fibrils by cell-derived extracellular matrix proteins, such as fibronectin or fibrinogen.

The viscoelastic reactions of cancer cells to the stiffness of collagen matrices have also been determined through analysis of intracellular rheology with optical trapping, and overall cell stiffness through the deformation analysis of cells, when released from the collagen matrix and pushed through a microfluidic channel (Wullkopf et al., 2018). In this study, alterations in both time-dependent intracellular viscoelasticity and total cell stiffness are detected in several cell lines. Comparing several cancer cell lines, it is concluded that cancer cells with high invasiveness can modify their stiffness in reaction to matrix stiffness, whereas less invasive cells do not react to stiffness.

\subsection{Hallmarks of the Protrusive-Type of Single Cell Migration}

Are cellular protrusions viscoelastic? To answer this question the viscoelastic characteristics of cellular protrusions has been explored using an optical tweezer setup (Khatibzadeh et al., 2013). The force-length diagrams connected with protrusions exhibit nonlinear proportions (Khatibzadeh et al., 2013). This finding is ascribed to the rupture of the bonds between the plasma membrane and cytoskeleton with subsequent flux of membrane lipids into the protrusion, and to additional viscous events due to slippage between the plasma membrane and cytoplasm encountered during the formation of a protrusion (Waugh and Bauserman, 1995; Hochmuth, 2000; Marcus and Hochmuth, 2002; Borghi and Brochard-Wyart, 2007). The existence of viscous interferences and bond breaks manifests itself as hysteresis in the reverse-pull examinations. The $\mathrm{W}_{\text {loss }}$ in control cells is near that onserved in human neutrophils (Xu and Shao, 2008). This analysis suggests the cytoskeleton dependence of energy loss in protrusion generation, as indicated in the contraction of the hysteresis circuit and the significant decline in $\mathrm{W}_{\text {loss }}$ in control cells following discontinuation of F-actin. The increased energy efficiency of protrusion generation in latrunculin-A treated cells is likely to be the outcome of less cytoskeletal-membrane connectivity in conjunction with the reduction in protrusion viscosity.

The Standard Linear Solid model matches the force-length diagrams in cells with intact F-actin and F-actin-destroyed cells, 
which leads to the suggestion of the viscoelastic response of the protrusions. To explore the viscoelasticity of the protrusions even further, the force relaxation of the protrusions has been investigated. For this purpose, in a reverse-pull procedure, protrusion pulling has been halted at the end of the pulling procedure, leading to an instantaneous relaxation of the protrusion force in a cell with intact F-actin. Force relaxation persisted to the point of reaching an equilibrium level prior to pushback. The presence of both force relaxation and hysteresis phenomena in these protrusions suggests the viscoelastic response of the protrusions ( $\mathrm{Xu}$ and Shao, 2008). The force relaxation curve has been fitted with the Standard Linear Solid model. The reduced levels of protrusion viscosity seen with F-actin disruption are in agreement with previous findings showing the dose-dependent diminishing impacts of F-actin breakdown on cytoplasmic viscosity (Marion et al., 2005; Tsai et al., 1994). The lower viscosity and more fluid response of the cytoplasm in F-actin-perturbed cells may be due to the lesser counteracting influences of the cytoskeleton microfilaments on the motion of the cytoplasm. Thus, the generation of longer protrusions before membrane-cytoskeleton disconnection in F-actin-perturbed cells and cholesterol-enriched cells may be attributable to their accelerated growth during the formation of the protrusion as a consequence of their lower viscosity levels. This result is in agreement with the simulations predicted by the Standard Linear Solid model, according to which protrusions with lower viscosity coefficient values are linked to longer lengths at the same pulling force. By the same means, protrusions with lower stiffness levels, seen in cells with F-actin breakage and cholesterol-enriched cells, are accompanied by longer protrusions for the identical force. Higher stiffness values of the protrusions either in the early phase of protrusion generation or towards the end of protrusion elongation, as induced for example by cholesterol depletion, cause shorter protrusions according to the Standard Linear Solid model. Consequently, the plasma membrane and cytoskeleton reciprocally participate in the viscoelastic response of the cellular protrusions. The effects of the mechanical characteristics of the plasma membrane are quite small compared to the actions of the cytoskeleton; however, unexpectedly, modulation of membrane constitution yielded significant modifications in the mechanics of protrusion.

The movement of cells can be subdivided into motility and migration. Motility is a spontaneous, undirected movement, whereas migration involves a directed movement occurring in reaction to a cell attracting or repelling agent (Kwon et al., 2020). However, many manuscripts today fail to take this into account and even confuse it. Cell movements extend from the uncoordinated rippling of cell boundaries to the migration of individual cells (Ridley, 2003) and to the collective movements of contiguous groups of cells (Friedl and Gilmour, 2009). Single-cell migration in the mode of protrusive migration allows cells to relocate to and between tissue compartments, a distinct process that is a key driver of inflammation-related leukocyte migration (Friedl and Weigelin, 2008). Specifically, cell migration is controlled by alterations in the cytoskeleton and the forming of focal adhesions. Cell migration comprises the subsequent set of events: firstly, protrusion of the leading edge, secondly, creation of focal adhesion at the front boundary and dislodgement at the trailing boundary, and thirdly, motion of the cell body (Murphy-Ullrich, 2001; Lindberg et al., 2008). In the course of migration, actin is polymerized at the leading boundary protrusion and repeatedly depolymerized at the trailing boundary. Polymerization and bundling of F-actin results in stiffening of the cells, whereas depolymerization softens the cells (De La Cruz and Gardel, 2015). Following actin polymerization at the protuberance at the cell front, adhesions are formed close to the front edge. The adhesions ripen by dynamic cross-linking of F-actin. The adhesions then dissolve at the trailing end when the connection between F-actin and integrin is disrupted. The physical interplay between F-actin and integrin delivers the pulling force required for cell migration. Finally, the hallmarks of "exertion of cellular protrusions," "cytoskeletal remodeling," which includes actin polymerization and cross-linking, "formation of focal adhesions," "disengagement of the rear end of the cell" and "generation of traction forces" can be stated for the individual cell migration (Figure 3).

The development of technologies to assess cellular elasticity, such as AFM and micropipette aspiration, indicates that elasticity and motility of cancer cells are mutually correlated, and elasticity has been acknowledged as a biomarker for the invasive potency of cancer cells (Wagh et al., 2008; Krause and Wolf, 2015). The relationship between cell elasticity and metastatic capacity has been established; however, several reports contradict one another (Kwon et al., 2020; Mierke, 2014; Mierke, 2019; Mierke, 2020). For instance, highly metastatic ovarian HEY A8 cancer cells are softer than non-malignant ovarian epithelial cells, and the migratory potential of HEY A8 is coupled to the restructuring of the actin cytoskeleton (Xu et al., 2020). Contrary, stiff breast MDA-MB-231 cancer cells have displayed excellent migratory capacity within dense culture matrix settings (Messica et al., 2017). The features of adhesion towards the extracellular matrix of cancer cells deviate from those of normal cells and are also linked with invasive and metastatic capacity of cancer cells (Khalili and Ahmad, 2015). Adhesion strength is commonly diminished in cancer cells, and the changes vary according to cell type and oncogene (Zou et al., 2002; Spangenberg et al., 2006). Adhesion strength is found to be heterogeneous in metastatic cells under stromal-like settings due to their enhanced sensitivity to $\mathrm{Mg}^{2+}$ - and $\mathrm{Ca}^{2+}$-driven breakdown of focal adhesions (Fuhrmann et al., 2017). When the cells adhere heavily to the extracellular matrix, their migration is abolished.

Despite this wide variety of migration modes, there seems to be a general consensus that all demand (to different extents) the below inputs: firstly, cell polarization, restructuring of the cytoskeleton, and generation and exertion of forces founded on the interaction between actin polymerization and contraction of actomyosin scaffold. Secondly, cell-cell adhesion and linkage through adherens-junction proteins that are connected the actin cytoskeleton. Thirdly, control through by chemical and physical cues. The fundamental functions realized by these various determinants endow cells with the capacity to generate forces, to adhere possible also in different ways to one another and to a substrate, and to be sensitive to mechanical and chemical cues. Nevertheless, it is still not fully understood how these fundamental characteristics are implemented during the migration of single cells. However, there exists an active self-regulation of the cytoskeleton. Assembly and disassembly of cytoskeletal scaffold and 


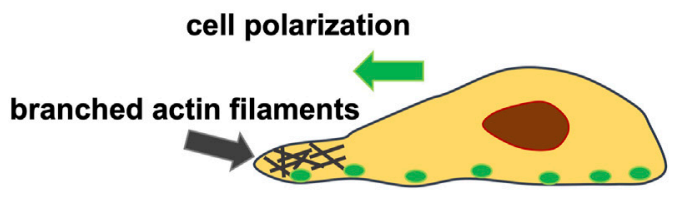

new adhesion new actin polymerization
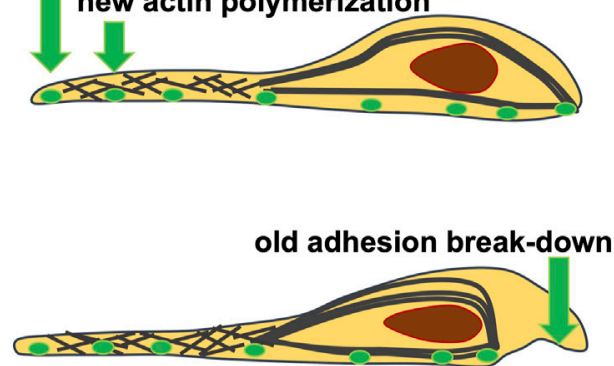

cell body translocation

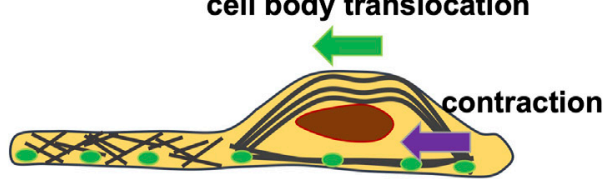

Hallmarks for single cell migration

1. Exertion of cellular protrusions

2. Cytoskeletal remodeling

3. Formation of focal adhesions

4. Disengagement of the rear end of the cell

5. Generation of traction forces

6. Adaption to environmental stiffness

7. Microenvironmental stiffness

FIGURE 3 | Hallmarks of single cell migration.

architectures are governed through multiple accessory proteins (Lauffenburger and Horwitz, 1996; Ridley, 2003). These regulating proteins constitute a reaction reticulum with several feedback mechanisms that enable the structures of the cytoskeleton to react to outside mechanical irritants (Marée et al., 2012, Marée et al., 2006). In addition, cytoskeletal structures, such as focal adhesions underneath integrins seem to fulfill a critical task in the spatiotemporal guidance of these regulatory proteins (Schwartz and Shattil, 2000).

Stem cell differentiation is guided by microenvironmental stiffness and thereby cells adapt to the stiffness of the substrate on which they are cultured and adhered to (Discher, 2005), which represents a hallmark of cell adhesion and migration. This hallmark can be termed "Adaption to environmental stiffness." In addition, cells move towards stiffer or softer environment (Pelham and Wang, 1997), which leads to another hallmark of cell migration. It can be stated as "Microenvironmental stiffness." All of these findings may also be transferable to cancer cells in the malignant progression of cancer.

\section{REGULATION OF TRANSCRIPTION LEADS TO CHANGES OF THE CELLULAR STATE}

There is strong compelling support for bidirectional mechanical signaling across the nucleus and the cytoskeleton. The mechanical characteristics of the nucleus have been the focus of mechanical analyses of cells for a while, especially when mutations in lamin $\mathrm{A} / \mathrm{C}$ appeared to underlie a number of genetic diseases with a mechanochemical foundation (Zwerger et al., 2011). The concept of mechanically linking the nucleus to the cytoskeleton has long been presented (Graham and Burridge, 2016). Electron microscopic analysis of cytoskeletal assemblies indicated that they appear to surround and be linked to the outer nuclear membrane (Capco et al., 1982). Tension applied to the cell surface results in nuclear distortion, corroborating that tension is transferred to the nucleus through the cytoskeleton (Gundersen and Worman, 2013). Moreover, the position of the nucleus varies according to cell type and is determined by interactions of the cytoskeleton (Gundersen and Worman, 2013). Great efforts have been made to pinpoint the proteins that link the nucleus to the cytoskeleton. Through a combination of techniques, the linker of nucleoskeleton and cytoskeleton (LINC) complex has been revealed (Crisp et al., 2006; Wang et al., 2009). The principal constituents of the LINC complex comprise members of the Nesprin protein family (Rajgor and Shanahan, 2013). They include transmembrane proteins that span the outer nuclear membrane and connect to SUN proteins in the intermembrane cavity between the outer and inner nuclear membranes. The SUN proteins span the inner nuclear membrane and connect to the nuclear lamins and other proteins, including emerin. As they elongate into the cytoplasm, the nesprins attach directly or indirectly to the actin, microtubule, 
and intermediate filament cytoskeletons (Rajgor and Shanahan, 2013). Imposing tension on isolated nuclei through tugging on the nesprins resulted in the stiffening of the nucleus, validating that the nucleus is mechanosensitive and reacts to tension conveyed over the cytoskeleton and the LINC complex (Guilluy et al., 2014). How can the nucleus, as a fairly stiff intracellular organelle physically linked to the cytoskeleton, influence mechanotransduction and facets of cell behavior, including cell migration? It is known that the tension imposed on the nucleus influences the transcription and the distinguished phenotype of the cells, which is shown below.

\subsection{Separation of the Link Complex}

There are two different strategies that can be implemented to separate the nucleus from the cytoskeleton: total excision of the nucleus (enucleation) and separation of the LINC complex through expression of dominant-negative KASH domains, dominant-negative SUN proteins, or exhaustion of LINC complex constituents (Crisp et al., 2006; Stewart-Hutchinson et al., 2008; Lombardi et al., 2011). The expression of dominant negative KASH domains, which can compete with Nesprin for the interaction with SUN proteins, causes altered mechanical phenotype of the cells (Stewart-Hutchinson et al., 2008; Lombardi et al., 2011). Rheological measurements showed reduced stiffness of the cytoplasm of transfected cells (StewartHutchinson et al., 2008), and changed force transduction through the cell and reduced deformation of the nucleus upon reaction to a surface force applied to the cell (Lombardi et al., 2011). Alterations in the perinuclear architecture of actin stress fibers have been observed. Moreover, disruption of the LINC complex in this manner also reduced the speed of cell migration and the directional persistence of cellular motility. The directional persistence may be due to altered cell polarity resulting from perturbation of the centrosomal/nuclear axis, which has been noted in reaction to the expression of dominant-negative LINC complex constructs (Lombardi et al., 2011). Reverse nuclear motion and centrosome realignment in cells migrating into a scratch wound is inhaled by the dominant-negative KASH domains (Luxton et al., 2010).

The nuclear lamina, which forms the basis of the inner nuclear membrane, is linked to the cytoskeleton through the LINC complex and is accountable for the majority of the stiffness and form of the nucleus. Deletion of the gene lamin A $\left(\mathrm{LMNA}^{-/-}\right)$showed that this lamin is especially relevant for these mechanical characteristics (citations...) (Broers et al., 2004; Dahl et al., 2004; Hale et al., 2008; Lammerding et al., 2004, Lammerding et al., 2006). In a similar manner to the expression of dominant-negative KASH domains, deletion of the lamin A gene impacts not exclusively nuclear mechanics, but cytoskeleton, cell polarity, and cell migration as well (Hale et al., 2008; Lee et al., 2007). Expression of lamin A mutant constructs similar to those involved in muscle diseases also recapitulated these findings (Hale et al., 2008; Folker et al., 2011; Zwerger et al., 2013). Even though no alterations in stress fiber organization occurred in the $\mathrm{Lmna}^{-/-}$cells, their focal adhesions seem to be shorter (Hale et al., 2008). The RhoA activity has been found to be decreased $\mathrm{Lmna}^{-/-}$cells
(Hale et al., 2008). RhoA activity has been seen to be reduced in cells that express a laminopathic Lamin A mutant, however, no alterations in the focal adhesions have been observed. This could be due to the fact that RhoA activity remained over a critical level of threshold.

\subsection{Removement of the Nucleus From Cells (Enucleation)}

Expression of compounds of the dominant-negative LINC complex or their genetic removal are accurate tools to examine the contribution of the connections linking the nucleus to the cytoskeleton. A much coarser attempt is the total excision of the cell nucleus. This is a crude approach but it has the benefit of destroying all links to the nucleus, whereas cutting the LINC complex can still keep intact other types of interactions of the cytoskeleton to the nuclear envelope. Largescale enucleation strategies for cells maintained in culture have been established in 1972 (Prescott et al., 1972). Enucleated cells, known as cytoplasts, have been noted to harbor multiple organelles and can last for hours or days, according to the cell type.

When techniques for enucleation first emerged, there has been just no examination in mechanotransduction. The question of whether the nucleus has any influence on the migratory behavior of a cell arose. It has been established that cytoplasts are able to migrate on glass, demonstrating that ownership of a nucleus is not a requirement for cell migration (Goldman et al., 1973). This finding has been validated in which the migratory properties of cell fragments without nuclei have been investigated (Shaw and Bray, 1977). In the same way, very small membrane-bound fragments of fibroblast cytoplasm (microplasts) exhibited several motile behaviors, including membrane rippling, filopodia elongation and retraction, and membrane blebbing behavior (Albrecht-Buehler, 1980). Investigating this phenomenon in more detail, such fragments have been seen to adopt either a non-polarized symmetric disk-like shape or a polarized arrangement. In the nonpolarized condition of the morphology, they exhibited no migration, while in the polarized condition they displayed persistent directional migration (Verkhovsky et al., 1999). Specifically, the directional migration in the non-polarized fragments can be induced by exerting a mechanical force toward one side. These previous investigations showed that a wide variety of cell types can perform efficient migration even in the absence of a nucleus. The possible role of the nucleus in the $3 \mathrm{D}$ migration of cells or the extent to which the nucleus participates in cellular mechanotransduction has not been analyzed. However, it has been pointed out that the nucleus has a major contribution to 3D migration (Wolf et al., 2013). Employing cytoplasts from fibroblasts or endothelial cells, it has been seen that they can migrate on two-dimensional (2D) surfaces (Graham et al., 2018). $\mathrm{n}$ addition, cytoplasts have been found to recognize gradients of growth factors and extracellular matrix and to undergo chemotaxis and haptotaxis. When examining migration in $3 \mathrm{D}$ collagen gels, cytoplasts exhibited minimal net migration. Nevertheless, the cytoplasts showed the ability to extend 
protrusions into the ambient $3 \mathrm{D}$ matrix without migration (Graham et al., 2018). Why are cells without nuclei able to migrate so well on $2 \mathrm{D}$ surfaces but so limited in $3 \mathrm{D}$ collagen matrices? In addition to the difference in dimensionality, another experimental distinction is that migration on two-dimensional surfaces is usually studied on very stiff substrates, whereas migration in $3 \mathrm{D}$ matrices uses relatively soft substrates. An explanation for the lower migration of cytoplasts in three dimensions arises from their lower capacity to migrate effectively on or in soft supports (Graham et al., 2018).

The impact of enucleation on the migration velocity of intact cells has been imitated by blocking myosin II activity with the inhibitor blebbistatin (Graham et al., 2018). Blebbistatin treatment of intact cells postponed the peak migration speed to stiffer substrates, indicating that enucleation may impair myosin activity and cell contractility. A similar effect of myosin blocking has increased the cell migration rate on soft substrata (Ulrich et al., 2009). This effect of blebbistatin on cytoplasts implies that excision of the nucleus impairs the overall contractility and mechanotransduction of the cells. Moreover, it has been shown that cytoplasts can only weakly contract collagen matrices and displayed decreased traction force on underlying medium in comparison to cells (Graham et al., 2018). Cytoplasts exhibited reduced stiffening due to pulling on magnetic fibronectin-coated beads. Thus, it can be concluded that excision of the nucleus decreased cell contractility and mechanotransduction (Graham et al., 2018).

What is the reason of reduced RhoA activity in cytoplasts? This needs to mirror either reduced GEF or enhanced GAP activity. This question has not yet been clarified. A possibility is that nuclear elimination depletes specific GEFs, such as ECT-2 and NET-1, that are localized in the nucleus. The extent to which these GEFs account for the total level of RhoA activity in cells is not clear. Another option is that a stiff nucleus connected to the actin cytoskeleton is a major driver of the total tension in the cell. As tension enhances the activities of multiple GEFs and conversely reduces GAP activity, reducing tension by excising the nucleus in this model is expected to lead to a decrease in RhoA activity. This is corroborated from the finding that cells missing lamin A have a soft nucleus, lower tension, and lower RhoA activity (Hale et al., 2008). In a similar manner, it has been inferred that the separation of the nucleus from the cytoskeleton due to the disconnection of the LINC complex leads to a reduction in the active RhoA (Thakar et al., 2017). Apart from the LINC complex and enucleated cells, the DNA itself can serve as a biomaterial scaffold (see above).

\subsection{Entanglement of Viscoelasticity, EMT and Governance of EMT Transcription Factors}

The entanglement of cells is required for cell movement and viscoelasticity seems to be a prominent player. The analysis of viscoelasticity is still focused on cell-cell adhesions rather than on the mechanical characteristics of individual cells. The latter seems to demand still more intensive research effort. However, it is well-known that the EMT relies on the deformability of cells. To account for the viscoelastic properties of cells, a threedimensional model has been built integrating the viscoelastic characteristics of the cytoskeleton and the membrane-cortex compound (Karcher et al., 2003). Apart from cellular viscoelasticity, the magnitude of the ambient viscoelasticity impact transcends cell response, and more than one research effort has demonstrated that extrinsic mechanical irritants result in alterations in gene expression. At the transcriptional and posttranscriptional levels, adherens junctions proteins have been identified to be functionally modified by transient and reversible EMT. Nevertheless, the mechanisms underpinning these alterations in cadherin levels and the interaction of environmental mechanics with EMT-related transcription factors are still just starting to be grasped. Transcription factor Zeb, Twist, and Snail families are EMT transcriptional regulatory agents that govern cadherin mRNA levels. Twist expression has been revealed to be mechanically adjusted during gastrulation of Drosophila, with exogenous compressive forces elevating the ectopic expression of twist (Farge, 2003; Desprat et al., 2008). Intriguingly, the subcellular homing of Twist has also been reported to be mechano-regulated in 3D-cultured MCF10A and Eph4Ras cell lines. The nuclear localization of twist can be determined when MCF10A and Eph4Ras cells are grown on rigid materials, nonetheless, within soft materials twist is primarily localized inside the cytosol (Wei et al., 2015). The EMT transcriptional regulator slug, which is a member of the snail family, has also been predicted to be mechanically modulated. A vimentinfacilitated mechanism of the mechanical stimulation of slug has been suggested (Liu et al., 2015a). The expression of E47, which is also an EMT transcription factor, and the nuclear targeting of SNAIL and its expression are also decreased during the impairment of the actomyosin contractility (Lee et al., 2011; Lee and Nelson, 2012). The underlying mechanism whereby mechanical cues are brought internal to direct the expression of these transcription factors is still an unresolved concern. Yap1, a compound of the Hippo signaling complex, is a prominent converter of mechanical environmental cues into biochemical pathways (Dupont et al., 2011; Calvo et al., 2013). Moreover, the Yap1 activity has been coupled to EMT, since Yap1 can largely interference with transcription factors that foster the transition of EMT. For instance, the EMT transcription factor ZEB1 turned into a transcriptional activator once it started interfering with YAP1 in more aggressive cancers (Lehmann et al., 2016).

Specifically, YAP and WW domain-containing transcriptional regulator 1 (TAZ), synonymously referred to as WWTR1 (together YAP/TAZ), are transcriptional regulators that react to various mechanical stimuli, encompassing extracellular matrix stiffness, cell form, and shear stress (Panciera et al., 2017). Although there is recent evidence for a type of crosstalk between YAP/TAZ and Notch during angiogenesis (Neto et al., 2018), the specific nature of the crosstalk's involvement is awaiting further exploration. Additional specifics have been elucidated in other tissues. During myogenesis, for instance, the initiation of contractions triggers the relocation of YAP to the nucleus, where it propels the expression of Jag2 and ensures 
Notch activation (Esteves de Lima et al., 2016), which is connected to the continuation of the progenitor cell stock necessary for regeneration (Bröhl et al., 2012), whereas YAP/ TAZ adjusts the expression of Notch-inhibitory ligands within epidermal stem cells (Totaro et al., 2017). The segmentation clock, a molecular oscillator that controls the correct segmentation of developing somites, combines YAP and Notch to link mechanical signals to molecular cues and synchronize cell performance across the segmenting tissue (Hubaud et al., 2017).

However, TGF $\beta$ interaction with SMAD proteins is critical for the activation of SNAIL, SLUG, and TWIST on a transcriptional level (Figure 4). The stability of this TGF $\beta$-SMAD complex has been revealed lately to be conveyed through Yap1. It is not yet clear how Yap1 can be targeted toward the nucleus and it has been proposed that force-driven opening of nuclear pores seems to be a major mechanism of Yap1 nuclear translocation (Elosegui-Artola et al., 2017) and possibly also of other transcription factors. These instances of interaction between mechanotransducers and EMT transcription factors highlight just a single of the many feasible mechanisms that migratory cells can utilize to convert mechanical cues into a molecular and cellular answer. These kinds of specific interactions can be the basis of other emergent features of collective migration that are related to cell-cell interfaces. Cell-cell adhesion has been revealed to be necessary for collective plithotaxis, a mechanism that imparts an "innate" directionality to cell monolayers (Trepat and Fredberg, 2011). Whereas the involvement of these canonical transcription factors in the control of EMT in vitro and in vivo is well settled, the involvement of mechanical interactions is comparatively less comprehended. Whether environmental viscoelasticity acts to modify EMT by influencing other facets of this intriguing event, such as apicobasal polarity, cadherin turnover, and MET, is just becoming unraveled.

\subsection{Role of Viscoelasticity in the Epithelial-To-Mesenchymal Transition of Single Cells}

Cellular viscoelastic characteristics are likely to fluctuate according to the biological state of the cells, for example, during the EMT transition in cancer, and thus viscoelasticity has the potential to be a beneficial physical biomarker (Nguyen et al., 2020). EMT represents an initial stage for individual cells and has originally been portrayed and defined as a process in which cells of a stalled epithelium shed their apicobasal polarity and cell-cell adhesion characteristics. Eventually, they become individual cells and migrate between tissues with a mesenchymal phenotype. On the length scale of the adherens junctions, a hallmark of this "canonical" EMT is a severe decrease in type I cadherin (E-cadherin) and, in a few cases, an augmentation of type II cadherins or the expression of type I cadherins with decreased adhesion strength, such as N-cadherin (Ruiz and Guenthert, 1996). Transcription factors of the Zeb, Snail, and Twist families are well-known as canonical upstream transcriptional regulators of this switch of cadherin (Figure 4) (Ansieau et al., 2014; Puisieux et al., 2014; Goossens et al., 2017).
This definition has been extremely informative in the initial phases to uncover the fundamentals of EMT, though most of this detail stems from experiments made in $2 \mathrm{D}$ in vitro settings and fails to consider the various tactics that cells and cell groups employ to migrate through divergent environments in vivo. The investigation of EMT has revealed that EMT may no more be handled as a linear, unidirectional event that switches the motility of single cells, but is instead now considered as a more dynamic event by which cells or sets of collectively migrating cells can accommodate themselves to the physical demands of their microenvironment. Several cues are known about the EMT. Firstly, EMT seems to be a reversible event and cells can also perform mesenchymal to epithelial transition (MET) (Jo et al., 2009; Kim et al., 2017). Secondly, apart the fact that EMT does not merely foster motility, it additionally aids cells to keep their stemness (Downing et al., 2013). Thirdly, migration can also take place even when cells display high amounts of E-cadherin ( $\mathrm{Li}$ et al., 2016). Fourthly, EMT not only enables individual cell migration, but also aids later in collective epithelial and mesenchymal migration (Campbell and Casanova, 2016). It can be hypothesized that the plasticity of individual and collective migration may be conducted by providing cells with tunable viscoelasticity features that then lead to alterations of their adherens junctions. The adherens junctions alterations take place at a transcriptional and/or posttranslational regime, through alteration of expression and breakdown dynamics of adherens junctions proteins (Barriga and Mayor, 2015). Moreover, these alterations have been linked to the new general network for EMT (Brabletz et al., 2018; Campbell and Casanova, 2016; Mierke, 2019; Nieto et al., 2016). Finally, the classical view on cell migration that is based on the hypothesis that cells can either migrate in a collectively manner as epithelial cells (Friedl and Gilmour, 2009; Rørth, 2009), or perform an EMT and migrate in a single manner as mesenchymal cells (Thiery et al., 2009). This traditional division into two distinct migration phenotypes is essentially based on the classical view of EMT as a dual binary choice entailing the switch from a completely epithelial to a completely mesenchymal state (Hay, 2005), and on the idea that a true mesenchymal cell migrates singly through the extracellular matrix (Acloque et al., 2009; Nieto, 2011). However, this classical view has been challenged in developmental biology because the rich diversity of migratory incidents that arise during animal development defy the simplicity of these definitions. Firstly, not only are there numerous intermediate situations in which migrating cells exhibit a mixture of epithelial and mesenchymal characteristics, although it is apparent that these are present many more times in vivo than heretofore assumed (Nakaya and Sheng, 2008; Shook and Keller, 2003). Secondly, it is clearly evident now that mesenchymal cells migrate frequently, demonstrating the level of coordination and cooperation that is attributed to collectively migrating cells (Scarpa and Mayor, 2016; Theveneau and Mayor, 2011). To account for these general observations, the distinction between individual and collective cell migration has progressed away from highly rigorous to more comprehensive or loose definitions (Rørth, 2012; Theveneau and Mayor, 2011). In line with this, any effort to categorize migration 


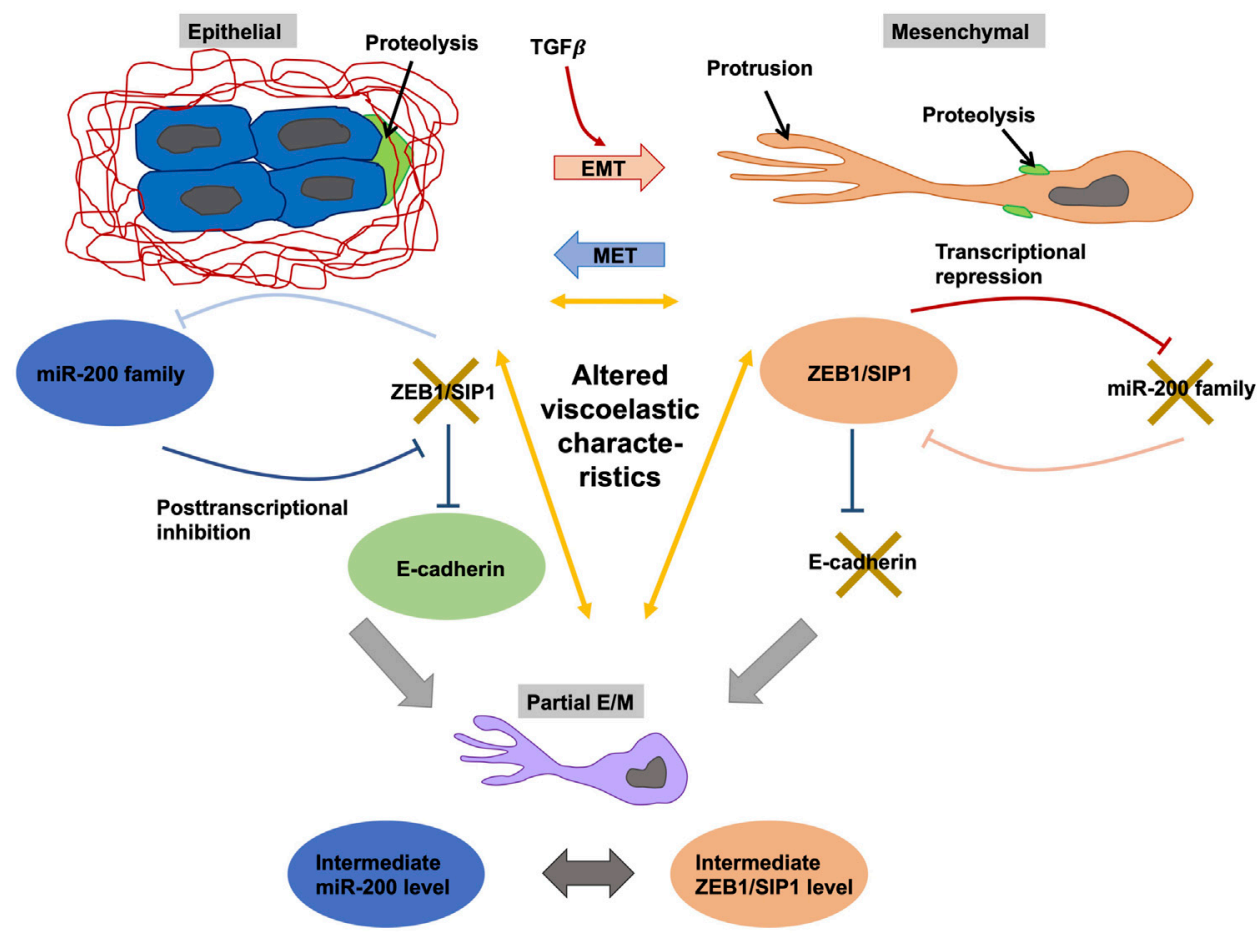

FIGURE 4 | Interplay of transcription factors and mechanical characteristics in EMT and partial EMT.

events and thus derive parallels between various systems presently demands the application of strict definitions with a large number of exclusions or the stamping of new and not very well defined notions such as that the cells migrate together one at a time (Friedl and Gilmour, 2009; Rørth, 2009) or cells perform a pseudo EMT (Pastor-Pareja et al., 2004) or partial EMT (Figure 4) (Tripathi et al., 2020).

Therefore, a different perspective can be proposed. It can be hypothesized that collective and individual as well as epithelial and mesenchymal cells have distinct and independent properties that come together to divergent degrees, not only at distinct migratory events but even at various time points within a single migratory effort. Correspondingly, it is suggested that in vivo cell migration phenotypes cannot be divided into distinct and mutually exclusive morphological classes, but rather should be considered as a general continuum of morphological diversity that can be accomplished by a combination of different and complementary mechanisms (Campbell and Casanova, 2016).

Cancer cells can themselves alter their viscoelastic properties to counter the microenvironment through adaptation of adherens junctions throughout collective invasion. To perform this adaption, cancer cells need to employ multiple strategies, including the phenomenon that they can switch between two morphological phenotypes, such as epithelial shape and mesenchymal shape or even display intermediate forms in the course of breast cancer invasion. Moreover, cancer cells are hypothesized to intrinsically regulate viscoelastic characteristics in order to modify their cell state transition rate and efficacy of cell movement. However, there is quite a lot more research required to confirm this hypothesis experimentally or refine it theoretically through the development of models.

In specific, these cancer cells are transformed through EMT in a metalloprotease-dependent fashion and in the next step the transcription factors contribute to the transition of cadherins to further restrengthen EMT. Firstly, secreted MMP-3 metalloprotease cuts the extracellular domain of E-cadherin that lowers the adhesion strength between cell-cell junctions. Secondly, migration of the cells is based on the activation of Rac and the EMT transcription repressor Snail that lowers additionally the amount of E-cadherin in the cells (Radisky et al., 2005). This facilitates collective migration in liquid mode with fewer stringent cell-cell contacts typically conveyed through N-cadherin or L1CAM (Yano et al., 2004; Gavert et al., 2007). EMT is reversible in cancer cells, although MET has been suggested to assist mesenchymal circulating cancer cells in the development of secondary tumors (Kim et al., 2017; Li et al., 2010). Moreover, impairment of the EMT-driven transcription factors Prrxl (Ocaña et al., 2012) or Twist (Tsai et al., 2012) may result in the activation of the MET switch. In this regard, MET endows cancer cells with robust adhesion that permits them to cease migration, aggregate, proliferate, and grow more aggressive.

In this perspective, it seems evident that although single-cell migration demands a peak of fluidity and a minority of cell-cell adhesion strength, collective migration proceeds in an optimal condition in which cell clusters can migrate either as monolayers of epithelial cells or as highly dynamic mesenchymal clusters. These optimal settings are a function of the microenvironment and come about when cells achieve the proper equilibrium of cell-cell 
adhesions and fluidity in a machinery imparted by a customizable EMT regimen. In vivo, the strength of cell-cell adhesion is not invariably adequate to sustain the collectivity of mesenchymal migratory clusters, and complementary mechanisms aid cells to migrate in a collective manner. Mutual attraction and restriction have been found to retain collectivity throughout collective mesenchymal migration out of the neural crest. In neural crest cells, mutual recruitment is generated through chemotaxis toward C3a released from the neural crest, which simultaneously expose the receptor C3aR (Carmona-Fontaine et al., 2011). In contrast, entrapment is imparted at least by the proteoglycan versican (Szabó et al., 2016). The concert of matching cell-cell adhesion with other environment-related and endogenous determinants permits cells to reduce their level of cell-cell adhesion to a baseline level to achieve fluidity and to migrate over exceptionally harsh constraints while maintaining collectivity. The following describes the viscoelasticity in multicellular processes, such as collective cell migration.

\section{VISCOELASTICITY IN MULTICELLULAR PROCESSES}

\subsection{Collective Migration}

Investigations employing in vitro and in vivo systems revealed that collectively migrating cells are able to utilize type-I cadherins in their adherens junctions to move within a collective mode through epithelia (Nelson et al., 2006; Wolf et al., 2007; Ewald et al., 2008; Czirók et al., 2013; Shamir and Ewald, 2015). It has been seen that collective migration is also feasible for mesenchymal cells. For example, N-cadherin-facilitated adherens junctions, permit neural crest cells inside clusters to assembly transient and flexible adhesions to migrate at an accelerated rate of adjacent interplay and fluidity, whereby the collective mode is maintained (Theveneau and Mayor, 2012; Theveneau and Mayor, 2013).

\subsubsection{Viscoelasticity Drives Cell Migration and Invasion} Random cell migration pertains to the intrinsic capacity of cells to migrate, often referred to as cell motility. Random walks can be ubiquitous be identified in biology (Berg, 1984). In specific, the movement of cells without symmetry-breaking gradients has long been characterized using random walk statistics (Wu et al., 2014). This basal random cell migration state opposes directional cell migration, in which cells migrate in the general direction of a chemical or physical signal. Contrary to Brownian particles, randomly moving cells display directional persistence, which means that they are far more probable to maintain motion in the previously adopted direction than to alter it, even when this direction is picked at random in an isotropic setting. The directed cellular migration can be analyzed by advection diffusion theory (Simpson et al., 2017).

In vivo, cancer cells are confronted with directional signals that are both soluble, such as gradients of growth factors, and nonsoluble, such as the orientation of collagen fibers. Based on the number and strength of such signals, the cells migrate randomly or in a targeted manner. Moreover, these mechanical signals may also trigger the functions of cells, such as proliferation (Esmaeili Pourfarhangi et al., 2018). In this context, it is ambiguous whether cell cycle progression impacts cancer cell migration and whether this impact is distinct in random or in directional migration. Directional migration is referred to as alignotaxis, topotaxis or contact guidance, which are used synonymously (Esmaeili Pourfarhangi et al., 2018).

$3 \mathrm{D}$ migration is not random. Both $2 \mathrm{D}$ and $3 \mathrm{D}$ migration exhibit a non-Gaussian exponential distribution of mean cell velocity, mainly due to cell-to-cell fluctuations (Wu et al., 2014). In contrast to the $2 \mathrm{D}$ scenario, $3 \mathrm{D}$ cell migration is inherently anisotropic: speed profiles exhibit varying rates and selfcorrelation processes in multiple directions, so that the classical persistent random walk (PRW) model of cell migration is insufficient. The inclusion of cell heterogeneity and local anisotropy in the PRW model can forecast 3D cell motility across a large range of matrix densities, thereby identifying density-independent nascent migration characteristics. This analysis also illustrates the unanticipated rugged link between cell speed and persistence of migration across a broad array of matrix densities (Wu et al., 2014).

Different types of migration-inducing cues lead to different migration modalities. However, during all types of directed migration the same four pillars of directed migration can be found (Figure 5). In specific detail, directed migration of cells has been found to be governed by a range of external incentives, varying from gradients of soluble (known as chemotaxis) to bound (known as haptotaxis) molecules. In complement to molecular gradients, mechanical property gradients (duro-/ mechanotaxis), electric field gradients (electro-/galvanotaxis), and ambient topology pattern distortions (ratchetaxis) have also been found to be capable of directing cell migration. Because cells migrating in vivo are subjected to a more challenging milieu consisting of a convoluted set of biochemical, biophysical, and topological hints, it is highly improbable that cell migration is governed by a single type of gradients. As many molecular actors are implicated in the cellular answer to these affecting signals, they are frequently been recycled and act as sensors or transmitters of both biochemical and biophysical events. Xenopus neural crest cells are introduced and compared with the performance of other cell types to argue about the significance of organizing cell guidance mechanisms into different categories. In addition, it is highlighted that although the examination of single affecting cues is instructive, the real harsh reality is that cells migrate through a kind of "mixotaxis" in which they incorporate and orchestrate multiple interventions through common molecular effectors to assure the resilience of the directed cell locomotion (Barriga and Theveneau, 2020). Is the concept of mixotaxis universally applicable to all cell types?

Identifying a way to induce directional cell migration is straightforward. An external signal that the cells can translate needs to be physically arranged. Cells can thereafter employ this input to create a forward-backward polarity that permits directional locomotion following this input. There are multiple signs, including chemical, electrical, mechanical, and topological, that can be ascribed to accomplish this task in supervised and 
Four pillars of directed migration

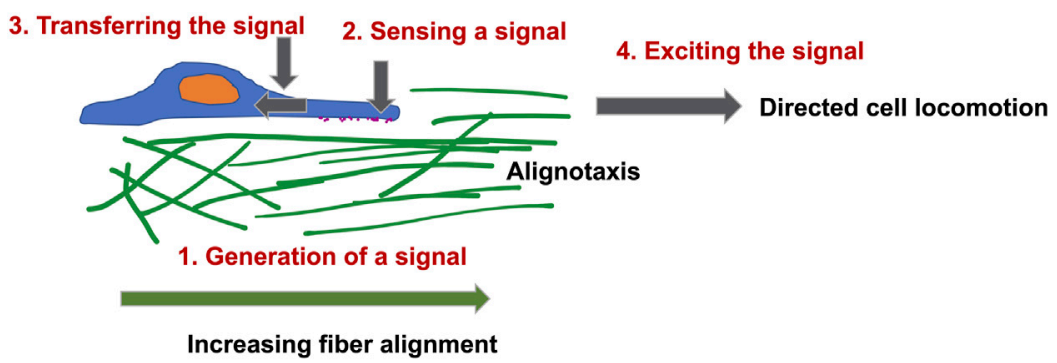

FIGURE 5 | Four pillars of directed migration of cells.

oversimplified experiments (Capuana et al., 2020; Zhao et al., 2006; Zhu et al., 2020). Since living systems are not designed to accurately follow distinct specifications in a timed manner that are controlled through by external checkpoints, under in vivo conditions, migrating cells are generally subjected to multiple inputs that are not hierarchically engineered and may even counteract to one another. Nevertheless, the migratory behavioral reaction of cells to such convoluted surroundings is yet consistent with logic. Moreover, any polarity evidence produced might not be as orderly orchestrated as it is in an in vitro array. In addition, certain cells can exhibit specific migratory patterns whereas their adjacent tissues may not. Therefore, collaboration, orchestration, and/or rivalry may arise between directionally migrating cells and the operations of their adjacent cells. Moreover, the same piece of input can lead to differing answers in diverse cell populations within the selfsame time frame, indicating that directional cues are not borne by the input itself but are instead produced as a consequence of the interference between cells and a particular input signal or series of signals. Thus, this can be analogized to how geneticists consider phenotype to be a consequence of the interactions between a genotype and an organism's surrounding local environment. For cells to be prepared to engage in directed migration, it depends on two simple facts: First, cells need to drive themselves, and second, they require the establishment and maintenance of forward-backward polarity. In other words, all inputs need to be slightly incorporated by a cell for directional performance to result. Additionally, in clusters of cells, intercellular exchange can result in new characteristics, such a cluster of cells may behave differently than a solitary cell in a similar setting (Theveneau et al., 2010). Therefore, uncovering the mechanisms that govern directed cell migration in all its intricacy may have myriad ramifications for how to understand the complicated morphogenetic processes at play. Beyond that, a focus on a more integrative model of directed cell migration approach could aid in developing efficient routes to prevent cancer metastasis, enhance wound healing, or assist in new techniques for ex vivo organ sampling in the framework of regenerative medicine.

\subsubsection{Role of Cell-Cell-Junctions in Collective Migration Mode}

Viscoelasticity can be tailored during collective migration of cells, whereby the adherens junctions play a key role. Intracellular pathway signaling activities are generally heterogeneous across the community of cells. The signal transduction process of population cells, normally random, is orchestrated through matching the fluctuating external inputs to their natural frequency (Tay et al., 2010) or through cell-cell exchange to achieve synchronization of their answers (Sun et al., 2012). For instance, collective calcium reactions in a densely packed cell population have been shown to occur when cells engage in gap junction communication in response to ATP pacing, resulting in more rapid, more synchronized, and highly corralled reactions relative to individual cells (Sun et al., 2012).

Inside the crowded cellular settings of biological tissues, the local tissue structure (Box et al., 2019) and the locomotion and deformation dynamics of single cells (Pan et al., 2016) produce propagating physical events that influence intracellular signaling pathways. The physical nature of the cellular microenvironment impacts long-term cellular behaviors related to cell fate, encompassing the polymerization of cells (Asnacios and Hamant, 2012; Copos and Mogilner, 2020), cell division (Bosveld et al., 2012; Gudipaty et al., 2017), and cell differentiation (He et al., 2018). However, the regulatory implications of mechanical stimuli on short-term cellular behaviors, including immune reactions, have not yet been elucidated extensively. Abnormal stiffness of the extracellular matrix has been demonstrated to elicit intracellular architectural alterations at the cytoskeleton-membrane interface, which in turn inhibits transient signaling replies to target-specific pharmaceuticals (Frangogiannis, 2016). The fibroblast-collagen matrix can release soluble cytokines that engage intracellular signaling transduction pathways when subjected to external mechanical force (Wells and Discher, 2008). These findings imply that dynamic mechanical inputs that arise from intercellular communications can drive intracellular responses. How mechanical signals aid cell adjustment to the constantly evolving chemical milieu and the 
relationship between collective cell performance and optimal tissue performance are still unclear.

Recently, this hypothesis has been explored. At a given frequency of addition of specific drugs to individual cells, chemical perturbation is converted into dynamic intracellular mechanical signals through RAC1-facilitated induction of dynamic cell-cell junctions and cytoskeletal rearrangements that act in synergy with chemical cues to favor collective signaling behaviors (Che et al., 2021). Perhaps the reverse is also conceivable: When the mechanical dynamics of the environment, including viscoelasticity, are altered, the cells may be able to react.

Beyond the hypothesized impact of intracellular structures to the overall viscoelastic character of cell, cell cluster and tissues, the cell-cell junctions and their coordination during collective cell migration may fulfill another crucial task. Emerging advances are broadening and expanding knowledge of the impact of cellular viscoelasticity throughout individual cell migration and how these characteristics can be adapted by cells to travel actively in restricted volumes. However, during collective cell migration, such mechanisms must be aligned not merely to confer the same directionality and velocity of migration to cells within the cluster, but also to permit the cluster to alter its viscoelasticity and meet the physical constraints of the microenvironment as a supracellular entity. To orchestrate these operations, cells within a migrating cluster have to link mechanically and build up efficient transmission channels to pass key messages from their surrounding environment across the cluster (EtienneManneville, 2011; Inaki et al., 2012). Cell-cell junctions are central in brokering these activities, and the fine-tuning of cell-cell adhesion strength has been linked to the fluidity of collectively migrating cells (Kuriyama et al., 2014; Cai et al., 2016) as well as their resistance to deformation as they move through constrained microenvironments (Cai et al., 2016). Adherens junctions and gap junctions are both fundamental in performing cell-cell communication by providing mechanical linkages, signaling and diffusion of signals via the channels. Moreover, the composition of adherence junctional complexes and the linkage to cytoskeletal filaments may provide different viscoelastic characteristics of the cells. Both adherens junctions and gap junctions react to mechanical stimuli and may therefore both be involved in the viscoelastic nature of the cells, cell clusters and tissues. Myosin-dependent contractility of actin cytoskeleton contributes to the organization and reinforcement of adherence junctions at intercellular contact sites (Krendel et al., 1999; Leckband and de Rooij, 2014). The activity of gap junctions builds a specific element of the adhesion complex, the adhesome, that couples the intercellular forces in the course of a collective migration of cells (Bazellières et al., 2015) and connexins, which represent the key elements of gap junctions and are known to act as mechanosensitive molecules (Bao et al., 2004) and may also contribute to viscoelasticity. Even though cells can be mechanically and molecularly connected through adherens junctions, gap junctions, and tight junctions (Tsukita et al., 2001; Kishikawa et al., 2008), the focus below lies on the most prominent adherens junctions as drivers of cell coupling and viscoelasticity during collective migration.
There is growing knowledge that the preferred migration mode in vivo seems to be the collective migration mode of cells. In collective migration, cells employ adherens junctions to mechanically link one another, which is critical for intercellular signaling processes that govern collective performance and possibly contribute to viscoelastic properties of cells. Adherens junctions perform a key function in facilitating the mechanical feedback loop within migrating cell clusters and their local environment via the coupling of force perception and transmission within the cluster and the transfer of tractions to the migrating substrate (Cavey and Lecuit, 2009; Harris et al., 2014; Kishikawa et al., 2008; Sluysmans et al., 2017). Hence, clusters may utilize various techniques to adapt their viscoelastic characteristics through precisely regulating their adherens junctions when migrating in their natural microenvironments toward altered microenvironmental mechanical cues.

\subsubsection{Fine Tuning of Cellular Viscoelasticity by Intracellular Factors}

The nature of collective migration of cells has been seen to rely to varying degrees on the density (Gudipaty et al., 2017; Tlili et al., 2018) and motility (Bi et al., 2016; Hayer et al., 2016) of cells and intercellular adhesion (Benjamin et al., 2010; Vedula et al., 2012). For instance, in a confluent cell monolayer, an augmentation of cell motility can lead to a transition from solid to liquid (Hayer et al., 2016; Malinverno et al., 2017), whereas a breakdown of intercellular links can result in random uncorrelated cell movements (Benjamin et al., 2010; Vedula et al., 2012).

Apart from intrinsic characteristics of cells, extrinsic signals, including geometric confinement (Camley et al., 2014; Doxzen et al., 2013; Li and Sun, 2014; Lin et al., 2017; Segerer et al., 2015; Tanner et al., 2012; Vedula et al., 2012), chemical factors (Harris et al., 2012) and electric field (Cohen et al., 2014) can additionally impact dynamic properties of cells. Migrating cells in vivo are frequently constrained geometrically by the surrounding environment, such as extracellular matrix or other cells. Typical cases are the invasion of cancer cells within the porous peritumoral stroma (Friedl et al., 2012) and the migration of border cells within the ovaries of Drosophila (Montell et al., 2012).

The main physical constraints faced by migrating cells in vivo are adhesion (friction), boundary, rigidity of migrating substrates, shear flow of extracellular liquids, topology and density of the ambient tissue or extracellular matrix scaffold (Charras and Sahai, 2014). To address these physical constraints of a 3D microenvironment, individual cells quickly change their viscoelasticity to recontour and "squeeze" or withstand deformation (Mueller et al., 2017; Petrie and Yamada, 2012). Nonetheless, to modify their viscoelastic characteristics and face their physical migratory microenvironment as a supracellular entity, cells within migratory aggregates must orchestrate the machinery that accomplishes such transformations.

How can mechanical signals modify cell-cell adhesions? The closest and most direct interplay between mechanical force and Notch signaling takes place during the receptor activation phase, which is a sequence of cleavage events to liberate the NICD from the membrane. During this event, a concealed peptidase binding 
site in the extracellular negative regulatory domain of Notch is uncovered through a conformational unfolding process step (Gordon et al., 2015; Chowdhury et al., 2016; Morsut et al., 2016). Unfolding is triggered through a traction force exerted on the receptor, which is imparted through ligand endocytosis following receptor binding. This endocytic traction force is reliant on dynamin, epsin, and actin (Park et al., 2016; Seugnet et al., 1997). Epsin directs ubiquitylated ligands for clathrin-facilitated endocytosis and participates in bending of the plasma membrane (Langridge and Struhl, 2017). Dynamin creates a helical multimer that provides the force to clamp the vesicle, while actin is indispensable for producing enough force to propel endocytosis of the ligand-receptor group (Meloty-Kapella et al., 2012). The capacity of the ligand-receptor pair to resist traction relies on its molecular affinity and load-carrying capability. Curiously, the Notch ligands jagged 1 (Jag1) and delta-like ligand 4 (Dll4) exhibit distinct mechanical characteristics. The attachment of Jag1 and Dll4 to Notch1 results in catch binding, which is a type of binding that strengthens when placed under tension. The tension necessary for the activation of Notch is distinct for the two ligands, such as $4 \mathrm{pN}$ for Dll4 and $12 \mathrm{pN}$ for Jag1, and it has been speculated that this is attributable to an enhanced binding rate of Dll4 (Luca et al., 2017). These two ligands possess different functions, including angiogenesis, inner ear development and differentiation of the airway (Benedito et al., 2009; Petrovic et al., 2014; Stupnikov et al., 2019). The differential force magnitudes needed for Notch activation thus might enable discrimination between ligands.

Is there a direct interplay between cytoskeletal or contractile force, the activation of Notch receptors and finally cell locomotion? There exists a direct interplay between cytoskeletal or contractile forces and Notch activation. Blocking nonmuscle myosin II to decrease contractility in signal transducing cells diminishes the activation of Notch in vivo (Hunter et al., 2019). This decline is irrespective of the appearance of ligands or receptors on the cell surface and summative with the action of dynamin or epsin blocking, suggesting separate inputs of contractile and endocytic forces (Hunter et al., 2019).

Like intracellular forces, external forces also seem to exert an activating impact on Notch. Specifically, the timing of streaminginduced activation of Notch indicates a direct involvement of Notch in the perception of shear forces, as NICD levels rise as soon as 30 or $60 \mathrm{~min}$ after the initiation of shear forces (Masumura et al., 2009; Fang et al., 2017). However, this fast initiation may be inhibited through vascular endothelial growth factor (VEGF) receptor 2 (VEGFR2, synonymously referred to as KDR) blockade, which may reflect the engagement of a mechanosensitive complex and also a canonical VEGF-driven downstream signal transduction pathway (Lawson et al., 2002; Masumura et al., 2009; Coon et al., 2015). Rapid activation, nevertheless, continues to enable transcriptional to posttranslational control point regulation of Notch ligands (Shimojo et al., 2008). Indeed, the mechanoresponsiveness of the Notch transmembrane domain has been shown to be restrained by knockout of Dll4 or hindrance of endocytosis, implying that regulation of Dll4 could govern this mechanoresponsiveness (Polacheck et al., 2017). Evidence suggests that the mechanosensitive channel Piezo1 may coordinate the activity of $\mathrm{Ca}^{2+}$-sensitive ADAM10 in interfering with the cleavage-driven activation of Notch through $\mathrm{Ca}^{2+}$ ions, but decoupling this enhanced S2 cleavage from enhanced ligand generation is difficult (Caolo et al., 2020). Because of these reasons, the evidentiary support for direct notch activation through shear stress or strain is still inconsistent. A lot of interferences between Notch and mechanosensors are indirect effects. It involves the mechanoregulation of ligand or receptor synthesis and the interaction between Notch and mechanosensors.

\subsection{Spheroid and Tumeroid Biology and Transition of Cell Collections}

Realignments are frequently characterized as either passive, occasioned from an external stress on the tissue scale, or active, for instance initiated and governed through an anisotropic dispersion of molecules, including myosin or cadherins, at cell-cell contacts (Guirao and Bellaïche, 2017). Active cell outline fluctuations are the pivotal components for fluidizing tissues to flow at the tissue scale when under stress, in in vitro (Marmottant et al., 2009) and in vivo settings (Mongera et al., 2018). Experiments on embryonic tissues (Serwane et al., 2017), multicellular spheroids (Marmottant et al., 2009; Guevorkian et al., 2010), or cell monolayers in the presense or absence of an underlying substrate (Harris et al., 2012; Vincent et al., 2015) proposed that tissues can be characterized as viscoelastic fluids. Nevertheless, there is a continuing dispute about the microscopic source and magnitude of the viscoelastic relaxation time $\tau$ and whether Madin-Darby dog kidney monolayers act primarily as liquids or solids (Vincent et al., 2015; Tlili et al., 2018).

In qualitative terms, flow modeling revealed that the cell deformation and rearrangement rate fields are strongly related in the absence of cell division and apoptosis (application of the pharmacological drug mitomycin). This is consistent with a simple Maxwell viscoelastic fluid model in which an elastic intracellular constituent is connected in sequence with a viscous intercellular constituent representing cell redistributions (Tlili et al., 2015). The overall deformation rate represents the strain rate, otherwise known as the velocity gradient. Specifically, this is the total of the coarse-grained cell deformation rate and the intercellular topological alteration rate, which is termed rearrangement rate. The change in relaxation behavior potentially provides a pathway to gradually shift from a developing, fluid tissue to a mature, firm and solid tissue (Mongera et al., 2018). Encoding memory of overall tissue flux in the cellular form is a conduit for transmitting knowledge from the tissue to the cellular level and, conversely, may affect intracellular signal transduction (Jain et al., 2013).

\subsubsection{Spheroid and Tumeroid Biology}

Commonly employed culture models include spheroids, which are well-formed aggregates of cells. As cancer model systems, 
tumor spheroids are capable of accurately replicating the core characteristics of solid human cancers. Spheroids and organoids are both $3 \mathrm{D}$ structures consisting of multiple cells. Although these terms are synonymously referred to, there are clear dissimilarities between them. Organoids comprise 3D stem cell models derived from either embryonic or adult stem cells. This kind of organoid can display self-regulatory capability, phenotypic characteristics of the organ from which they are cultured, and ambient physiological modeling via genomic changes. An organoid is a compilation of organ-specific cell types derived from stem cells or organ precursors that self-organize similarly to in vivo through cell sorting and spatially constrained lineage commitment (Lancaster and Knoblich, 2014). However, the multicellular tumor spheroid model has been first introduced in 1971 and acquired by culturing cancer cell lines in nonadherent environments (Sutherland et al., 1971). Tumor spheroids are a cancer stem cell expansion model; tissue-derived tumor spheroids and organotypic multicellular spheroids are generally harvested through mechanical disassembly and sectioning of tumor tissue (Weiswald et al., 2015). In principle, higher-order selforganization occurs in organoids than in spheroid cultures, and the first are more strongly reliant on a matrix for their emergence. In the following spheroid and tumeroid cultures have been frequently employed to analyzed and decipher the phenomenon of transitions in cell collections.

\subsubsection{Role of Viscoelasticity in Jamming to Unjamming Transition of Cell Collections}

The viscoelasticity of multicellular arrays relies on the wandering cell constitution and the velocity of its transformation, which proceeds by the transitions from the wandering to the resting cell condition and conversely (Pajic-Lijakovic and Milivojevic, 2019a; Pajic-Lijakovic and Milivojevic, 2019b). These crossings have been regarded as cell jamming state transitions due to disturbances (Angelini et al., 2011; Bi et al., 2016; Garcia et al., 2015; Nnetu et al., 2013; Oswald et al., 2017; Park et al., 2016). Multiple interdependent elements affect the jamming phase transitions such as: firstly, an elevation in the packing density of cells (Henkes et al., 2011; Nnetu et al., 2013), secondly, the adhesion energy of cell-cell contacts (Bi et al., 2016; Garcia et al., 2015), thirdly, amount of cellular forces and maintenance of these forces (Garcia et al., 2015), fourthly, cellular morphology (Garcia et al., 2015; Park et al., 2016), fifthly, contact inhibition of locomotion (CIL) (Zimmermann et al., 2016) (Figures 6A,B). The impairment of cell motility affects the condition of viscoelasticity and, subsequently, the cell jamming phase transition. In spite of substantial research aimed at investigating cell jamming, the process is still not completely comprehended from the viewpoint of rheology. Nevertheless, the mechanism(s) whereby this jamming condition occurs are linked to the viscoelasticity (Pajic-Lijakovic and Milivojevic, 2019b).

The classical material-science modification of the state of viscoelasticity is induced by the decrease of the agitation of system elements. The motion may be limited firstly, by raising the frequency in the oscillating flow field or reducing the observation time, secondly, by enhancing the concentration of system components and their stiffness, and thirdly, by lowering the temperature, such as the glassy state transition (PajicLijakovic and Milivojevic, 2021a) (Figure 6C). The apparent enhancement of the stiffness of the system components can be derived from the intensification of the field of interactions which are instantiated through the excluded volume concept (Neal et al., 1998). The rheological reaction of coacervate-based systems has been examined in the oscillating shear field at rising frequency, and four regimes have been identified. The first regime is a lowfrequency terminal regime that can be described through the Maxwell model (a viscoelastic liquid). The second regime represents a middle-frequency plateau regime that can be explained through the Kelvin-Voigt model (a viscoelastic solid). The third regime represents a higher-frequency transition regime (a viscoelastic solid). The fourth is a highfrequency jamming regime (a viscoelastic solid) (Figure 2) (Liu et al., 2010; Schroyen et al., 2020). There are minimally two or perhaps even three regimes (Stamenović, 2006). The final regime is typified by intense and disordered motion of the system elements and by in-chain reciprocal interactions, which leads to substantial energy dissipation. Plateau regime is equivalent to intense entropic forces generated due to interchain dynamics (Pajic-Lijakovic and Milivojevic, 2021a). The transition regime is connected to diminished mobility of the system components and explains local conformational alterations of the chain moieties influenced strongly through the entanglement relaxation time (Liu et al., 2006). The main features of the jamming condition are: firstly, migration of the system elements is largely damped, such that the diffusion parameter approaches zero, secondly, relaxation time goes to infinity, and thirdly, storage modulus $G^{\prime}(\omega)$ and loss modulus $G^{\prime \prime}(\omega)$ meet the requirement $G^{\prime}(\omega) /$ $\mathrm{G}^{\prime \prime}(\omega)=$ const $>1$ (Hunter and Weeks, 2012; Pajic-Lijakovic and Milivojevic, 2019a).

The density-facilitated cell jamming state transition in the course of collective cell migration has been broadly examined (Angelini et al., 2011; Bi et al., 2016; Garcia et al., 2015; Nnetu et al., 2013; Oswald et al., 2017; Park et al., 2016). Nevertheless, the relevant variation of the condition of viscoelasticity has not been incorporated. To fill this loophole, a systematic theoretical approach from a rheological point of view is offered. Specifically, it has been seen that there occurred the density-induced switch from a convective toward a conductive mechanism of collective cell migration (Angelini et al., 2011). Consequently, this transition alters the stage of viscoelasticity. In agreement with this, the velocity correlation length has been inferred to become dependent of the cell velocity (Garcia et al., 2015). In specific, the correlation length firstly raised with cell velocity for cell velocities less than about $1 \mu \mathrm{m} / \mathrm{min}$ and secondly declined with cell velocity for cell velocities greater than $1 \mu \mathrm{m} / \mathrm{min}$. These two tendencies of cell movement comply with distinct states of viscoelasticity. An even more advanced elevation of the packing density of cells evokes a decrease in cell mobility which materializes in subdiffuse cell migration that initiates the anomalous character of energy dissipation (Nnetu et al., 2013). Cell monolayers seem to act as amorphous solids at decreased cell speeds (Garcia et al., 2015). Each viscoelastic condition per regime ought to be typified by a suitable stress-strain constitutive model. For instance, the collective migration of cells causes the generation of stress, 


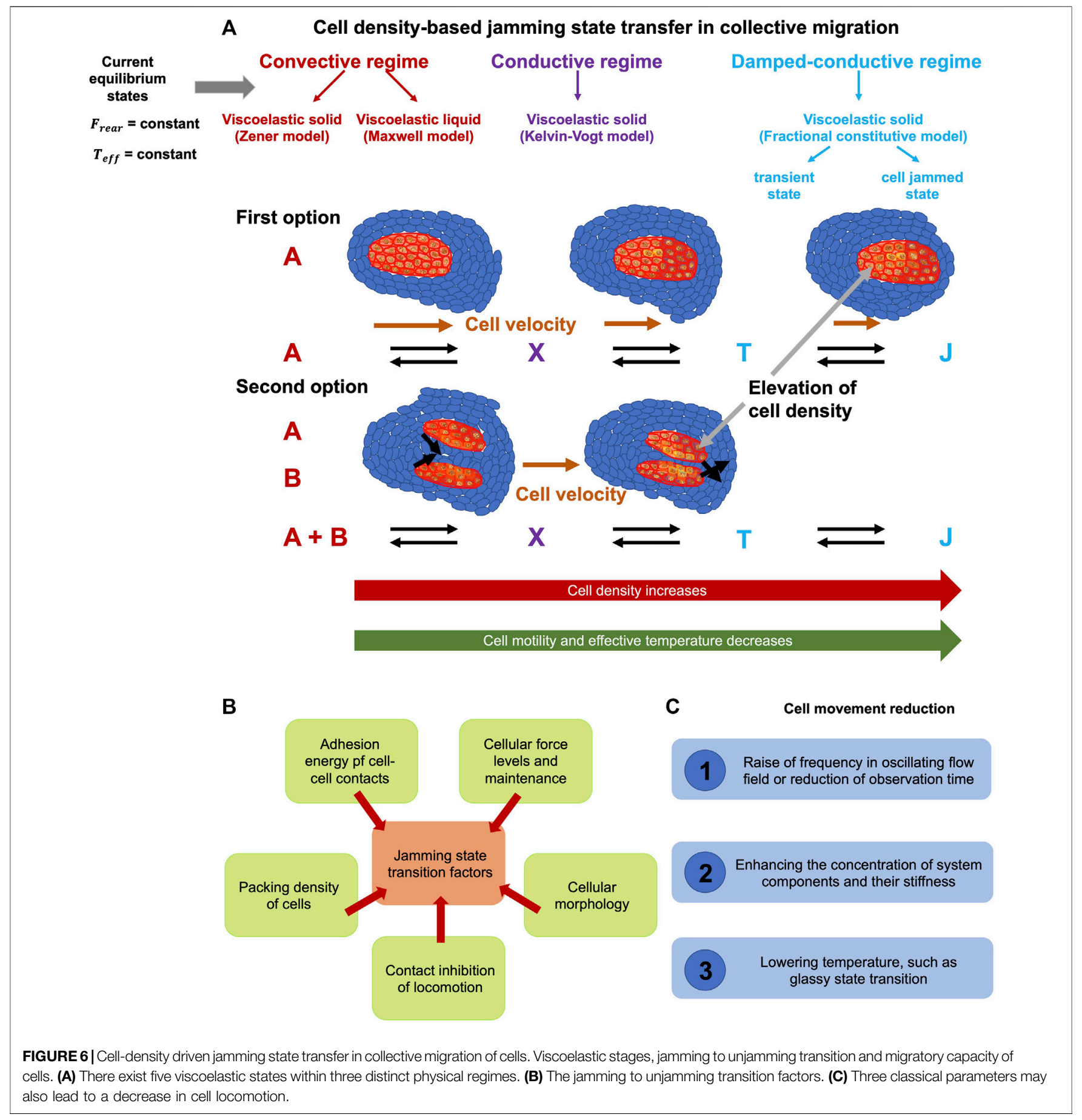

stress relaxation, and the buildup of remnant stress (PajicLijakovic and Milivojevic, 2019b, Pajic-Lijakovic and Milivojevic, 2020). The stress level ranges from normal including compressive and tensile to shear (Pajic-Lijakovic and Milivojevic, 2019a, Pajic-Lijakovic and Milivojevic, 2020). Normal stress is concentrated mainly within a core area of migrating cell clusters amassed during their motion through the crowded surroundings (Pajic-Lijakovic and Milivojevic, 2019b; Pajic-Lijakovic and Milivojevic, 2021a) and during clashes of migrating cell faces (Nnetu et al., 2012). Normal intrinsic stress accumulation is accountable for enhancing cell packing density (Trepat et al., 2009) and lowering cell motility (Pajic-Lijakovic and Milivojevic, 2021a), and subsequently, for the transition to the stagnant jammed state (Pajic-Lijakovic and Milivojevic, 2021b). The theoretical analysis incorporates: the organization of the established regimes through cell velocities, the degree of modification of the cell packing density and viscoelasticity on a supercellular scale. In the following, the 
emphasis is placed on an effort to elucidate the density-driven development of viscoelasticity and the state transition of cell jamming, and to highlight the factors governing the dynamics of long-term redistribution of cells, along with their interconnections.

\subsubsection{Impact of Stress on Viscoelasticity}

Collective cell migration encompasses stress generation, stress relaxation, and intrinsic stress build-up (Pajic-Lijakovic and Milivojevic, 2019a, Pajic-Lijakovic and Milivojevic, 2020). The allocation of normal, including compressive and tensile, and shear residual stresses has been quantified for multicellular $2 \mathrm{D}$ systems conducted under in vitro settings (Nelson et al., 2006; Serra-Picamal et al., 2012; Tambe et al., 2013). Thereby, the maximum stress is in the range of $100-150 \mathrm{~Pa}$ (Tambe et al., 2013). Normal residual stress build-up leads to an augmentation of the density of cell packing (Trepat et al., 2009). An elevation in cell packing density acts to trigger a decline in cell velocity (Angelini et al., 2011; Nnetu et al., 2013; Tlili et al., 2018) and, building on this, a shift in the condition of viscoelasticity.

Two specific scenarios of augmented packing density of cells can be assumed, one within migrating clusters, denoted A, caused by normal residual stress accumulation during their migration through dense environment and the other produced by colliding velocity surfaces, denoted A and B (Pajic-Lijakovic and Milivojevic, 2019b). Cell separation is disregarded on this temporal scale. Both settings can result in the switch from the migratory to the dormant and stalled cell state (Pajic-Lijakovic and Milivojevic, 2019a) (Figure 6). A and B signify distinct conditions of cell migration within a convective regime, $\mathrm{X}$ signifies cell migration occurring within the conductive regime, $\mathrm{T}$ signifies the transient condition of the cell encountered within the damped-conductive regime, and J signifies the condition of cell engorgement or jamming also experienced in the damped-conductive regime (Figure 6). Systems have to pass through a transitory mode before entering the perturbation mode, such as the jamming state (Baumgarten and Tighe, 2017). The fundamental mode of migration for the specific cell conditions marked $A$ and $B$ is convective, whereas the transient condition $\mathrm{X}$ represents cell movement through a conductive type of mechanism. Therefore, the three regimes are experimentally detected in collective migration of cell monolayers (Angelini et al., 2011; Nnetu et al., 2013; Garcia et al., 2015). The transient and perturbative (jammed) conditions comprise portions of this damped-conductive regime. For each regime, cell velocity, which is the degree of alteration of the cell displacement field evoked through collective cell migration, packing density of cells, and the respective constitutive stress-strain relationship define the regime. Conversions from convective to conductive modes and from conductive to damped-conductive modes are initiated through normal residual stress build-up with a concomitant rise in cell packing density (Pajic-Lijakovic and Milivojevic, 2021b). However, feedback crossovers from the damped-conductive to the conductive regime and from the conductive to the convective regime are induced through contact inhibition of locomotion (Zimmermann et al., 2016; Alert and Trepat, 2020). Contact inhibition of locomotion is accountable for the disorganization of multicellular systems and the attenuation of cell-cell adhesion interaction sites (Mayor and Carmona-Fontaine, 2010). Densitydriven transfers from one regime to another may be handled as viscoelastic phase transformations.

Raising the packing density of the cells lowers the effective volume per individual cell and intensifies cell-cell interactions on this way. The elevation of the cell packing density decreases the free volume, $V_{f}$, but the individual cell volume remains nearly constant throughout the collective cell migration. The jamming condition arises when the free volume is equivalent to the exclusive volume. This requirement reinforces the contact inhibition of locomotion, which can induce decongestion or unjamming (Alert and Trepat, 2020). The principle of excluded volume has been established in physics to characterize multibody interactions in a variety of soft systems of polymers and soft and stiff particles (Neal et al., 1998). The excluded volume is related to the interaction field and has been formulated from the second virial coefficient (Neal et al., 1998). This principle has been extended to dense cell populations to specify the minimum effective volume per individual cell equivalent to the jamming condition and, building on this, the peak cell packing density.

Significant efforts have been devoted to debating the densitydriven condition transformation of the cell jamming phenomenon occurring throughout the collective $2 \mathrm{D}$ cell migration. Cell jamming is a corollary of the decrease in cell motility induced due to normal residual stress build-up. Inherent stress accumulation impacts firstly, packing density of cells, secondly, cell-cell adhesion energy, thirdly, magnitude of cellular forces, fourthly, shape of cells, fifthly, contact inhibition of locomotion, and sixthly intricate interrelationships. The impairment in cell mobility affects the stage of viscoelasticity. However, the jamming phase switch is elusive from a rheological point-of-view.

Moreover, the gap needs to be filled through the clarification of the linkage between either viscoelasticity and packing density of cells or viscoelasticity and migratory capacity of cells. This densitydriven development of viscoelasticity keys in accounting for multiple crossovers among five viscoelastic modes obtained in three regimes: firstly, convective regime, secondly, conductive regime, and thirdly, damped-conductive regime. The convective regime contemplates two conditions of viscoelasticity in function of the amount of cell speed and the degree of cell-cell adhesion interactions. Increased cell velocities and faint cell-cell adhesion interactions (indicative of inferior cell packing efficiencies) yield distinct fluid-like characteristics delineated by the Maxwell model, whereas slower cell speeds and firmer cell-cell adhesion interactions (indicative of confluent multicellular regimes) assure solid-like characteristics delineated by the Zener model. The switch from convective to conductive mode is paralleled by a marked decrease in cell migration induced by the rise in normal inherent stress build-up. It is this phenomenon, referred to as the "plateau" mode in the vicinity of the system perturbation, such as cell jamming, that has been delineated through the Kelvin-Voigt model. A continued enhancement of cell packing tightness results in an anomalous character of energy dissipation throughout the 
collective cell migration as a feature of the damped-conducting mode regime. This regime incorporates two states of viscoelasticity, namely the cell transient condition and the jamming condition characterized through the fractional constitutive model. The process of viscoelasticity development is implemented by multiple current equilibrium conditions. Each equilibrium condition has been thermodynamically defined in terms of constant factors: on the one hand the Helmholtz free energy of cell restructuring and on the other hand, the effective temperature. The density-induced decrease in cell motility disrupts the equilibrium condition by lowering the effective temperature. The subsequent equilibrium condition accompanied by a new condition of viscoelasticity is determined for the new levels of effective temperature and Helmholtz free energy of restructuring events. Complementary experiments are needed to firstly relate the normal residual stress accretion to the variation of cell packing density, secondly reveal the cell number density and damping coefficient for the jamming condition of diverse cell types, thirdly identify the appropriate ingredients for the suggested constitutive models, fourthly outline the time evolution of the viscoelastic modification from the convective regime to the dampedconductive regime within several $2 \mathrm{D}$ and $3 \mathrm{D}$ experimental settings, and fifthly locate the required time span for the cells to pass through the unjamming transformation.

\section{VISCOELASTICITY OF CELLS VARIES DUE TO DISEASE STATE AND PROGRESSION}

There is agreement that there exist differences in viscoelastic characteristics of cells in the various diseases, such as cancer, fibrosis and inflammation. It seems to be likely that there are also pronounced differences of disease models, when the cells are cultured in $2 \mathrm{D}$ vs $3 \mathrm{D}$. The viscoelastic value of cells is no static number, it is rather dependent on time and is hence dynamically regulated. It is rather suggested to correlate with the progression of the disease, including the malignant progression of cancer. Moreover, the viscoelastic response can also be implemented, when investigating the reaction toward specific drug treatments, since it has been shown that 2D cell culture-based drug screenings turned out to be ineffective in predicting whether a drug treatment helps to treat cancer and its malignant progression. Even the chemoor radiotherapy approaches cannot be predicted due to altered tumor microenvironment and the broad heterogeneity of cancer types.

\subsection{Viscoelasticity in Cancer}

The transition of cancer cells from a benign phenotype to an invasive or metastatic identity entails both biological features, such as upregulation, downregulation, or inhibition of the expression of specific genes and tumor markers (Simpson et al., 2005), and physical features, such as structural alterations of cells and tissues that cover molecular and scaffold structures (Hall, 2009; The Physical Sciences Oncology Centers Network, 2013; Wirtz et al., 2011). In the last two decades, mechanical modifications of the phenotype of cancer cells have emerged as an integral piece of what has been seen as role of focus in cancer disease progression (Kumar and Weaver, 2009; Mierke, 2014, Mierke, 2019). From the liberation of cells from a primary solid tumor into the bloodstream via intravasation, as circulating cancer cells to their extravasation into surrounding tissues and their settlement in a new targeted organ, there are pronounced changes in the mechanical characteristics of the cells, particularly their adhesion and inherent rheology (Figure 7) (Kumar and Weaver, 2009). Comprehending cancer from the viewpoint of biomechanics may offer an alternative avenue to assess the outbreak or advancement of the disease. For instance, the rise in cell deformability corresponds in a direct way to the development of a transformed phenotype from a non-tumorigenic, benign cell towards a tumorigenic, malignant cell. The reduction in the amount of actin in the cytoskeleton and its overall structure is linked directly to the modifications in the biomechanical characteristics of the cells (Ketene et al., 2012). However, elasticity by itself is not typical of the entire mechanical nature of the cell. Cells exhibit viscoelasticity, i.e., they react in a time-dependent manner to an imposed force, which is due to interior frictional interfaces between the cell constituents and the organelles. In a 3D environment there are additional frictional interfaces between the cells and their microenvironment, which also has frictional interfaces within its own scaffold. Hence, it is expected that the viscoelastic characteristics of cells are altered by a $3 \mathrm{D}$ matrix scaffold. In line with this, when a $3 \mathrm{D}$ matrix is additionally cross-linked which leads to bundling of fiber and less friction, the matrix viscoelasticity is reduced and the material is more elastic which in turn increases the motility of cancer cells. The viscoelastic character of the cells renders them heavily reliant on the rate of deformation. The elastic modulus of breast cancer cell lines appeared to be governed by the loading rate during indentation (Li et al., 2008). However, to date, there have been several investigations on the viscosity of cancer cells using AFM. Analyses of cancer cell viscoelasticity can also provide a baseline for drug screening and therapy, since drug diffusion into cancer cells is partially a function of cell viscosity as medication molecules navigate their passage through the viscous cytoplasm (Kalwarczyk et al., 2011). A recent experimental study indicates that metastatic cancer cells rigidify upon active invasion and are completely imbedded in collagen inside a 3D environment (Staunton et al., 2016). This demonstrates the critical importance of determining the viscoelasticity of cancer cells in their suspended form while in the circulation of blood vessels during the invasion event. These findings suggest that the elastic modulus decreases significantly as the metastatic capacity of a cell increases from control human breast MCF-10A epithelial cells to noninvasive human breast MCF-7 cancer cells to highly invasive human breast MDA-MB-231 cancer cells (Fischer et al., 2017; Nematbakhsh et al., 2017). It implies that breast cancer cells soften as they progressively increase in invasiveness. Moreover, highly invasive breast MDA-MB-231 cancer cells can exert higher forces, as indicated by the absolute fiber displacement 


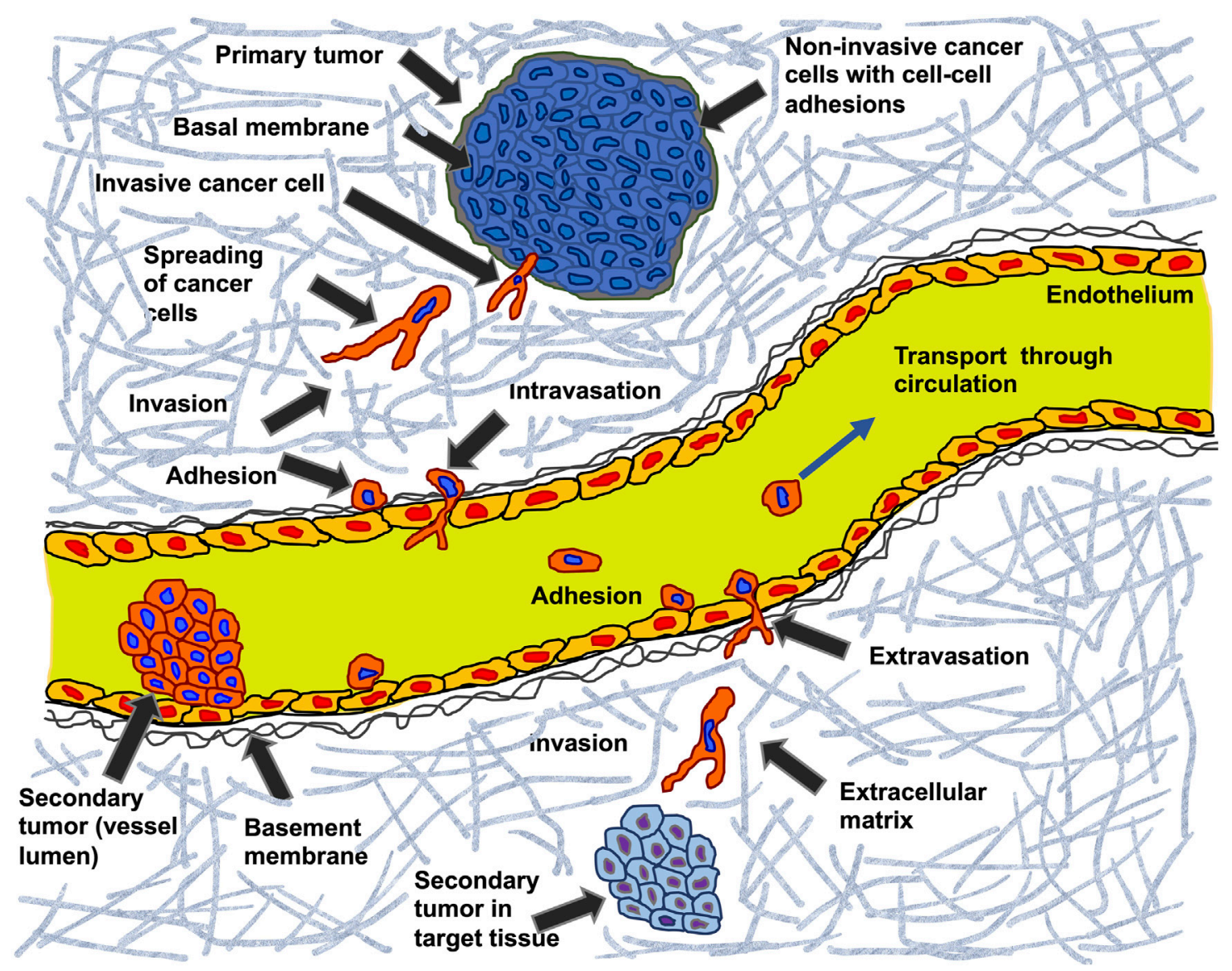

FIGURE 7 | Cancer metastasis is based on an accurately regulated and sequential order of events.

of 3D collagen fiber networks, compared to non-invasive breast MCF-7 cancer cells or control breast MCF-10A mammalian epithelial cells (Fischer et al., 2017).

Besides the investigation of single cells, cell spheroids seem to provide specific features that may help to understand what is happing in a solid tumor in more detail. For instance, spheroids can actually outgrow and produce their intrinsic extracellular matrix, and cell adhesion molecules, notably cadherins, such as E-cadherin, can arise to tether cells inside the spheroid. Several prior research efforts have centered on the specific impact of compressive stresses applied externally to the solid tumor (Helmlinger et al., 1997; Delarue et al., 2014), which had only a minor impact on spheroid growth. Fewer findings are available to support comprehension of the mechanical response of such spheroids, however recent modeling has centered on the flow of interstitial fluid inside the spheroids (Delarue et al., 2013) and has demonstrated that poroelastic active models can completely characterize them (Dolega et al., 2021). When low quantities of collagen (in this case between 0 and $0.03 \mathrm{mg} / \mathrm{ml}$ ) have been utilized, cells have been observed to reorganize themselves effectively to create a more condensed structure (Abidine et al., 2021), where collagen has been employed as a surrogate (Delarue et al., 2013; Elosegui-Artola, 2021). This led to several responses that can be attributed to the viscoelastic character of collagen (Vader et al., 2009; Iordan et al., 2010; Aermes et al., 2020). In fact, collagen has improved viscous characteristics at low frequencies and presents extremely small moduli that are typically a few tens or hundreds of $\mathrm{Pa}$ (Aermes et al., 2020).

How can poroelasticity and viscoelasticity be distinguished from each other? Poroelastic and intrinsic viscoelastic effects are both energy dissipation mechanisms, whereas the poroelasticity depends on the flow and viscoelasticity is flow independent. Poroelasticity has been demonstrated in connective tissues such as articular cartilage, which is comprised of a loadbearing and dissipative element over a wide-band spectrum of loading frequencies. Thus, cartilage has a heterogeneous structure consisting of a dense solid matrix with collagen fibrils and proteoglycans and a fluid (Mow et al., 1992). Here, the liquid is the largest component (approximately $60-85 \%$ of the wet weight) and is critical in swelling the interfibrillar cavity (approximately $30 \%$ of the overall water) and the extrafibrillar cavity (Torzilli, 1985; Maroudas et al., 1991; Mow et al., 1992). Cartilage dehydrates and rehydrates as a result of pressureinduced exudation of liquid through the solid matrix at ordinary loading rates in vivo. The time-dependent characteristics of cartilage are based on paired mechanisms involving the solid matrix and fluid flow. The mechanisms 
have been typified as poroelasticity and intrinsic viscoelasticity, leading to effective and persistent broadspectrum dissipative characteristics (Fulcher et al., 2009; Lawless et al., 2017; Nia et al., 2011, Nia et al., 2013).

Prior investigations have yielded indications of poroelasticity and intrinsic viscoelasticity of cartilage, although the respective relational contributions of the two remain ambiguous. The dissipation and response due to poroelasticity is based on the frictional interaction between the solid and the fluid, and hence relies on the flow (Nia et al., 2011, Nia et al., 2015). Poroelasticdriven dissipation is dominant at relatively small length scales (approximately 5-6 $\mu \mathrm{m}$ ) under oscillatory loading (Nia et al., 2011; Nia et al., 2013). Intrinsic dissipation powered by viscoelasticity is connected with a retardation caused by molecular friction and reorganization of a solid matrix (Nia et al., 2011; Nia et al., 2015) and is consequently independent of flow (Mak, 1986; June et al., 2009; Lai and Hu, 2017). The intrinsic viscoelasticity of cartilage has been analyzed with macroscopic compression experiments (Mak, 1986; Fulcher et al., 2009; June et al., 2009; Lawless et al., 2017) and measured with small magnitude shear loads (Henak et al., 2016). Although a handful of investigations have assessed poroelasticity (Nia et al., 2011; Nia et al., 2013) and intrinsic viscoelasticity of cartilage (Fulcher et al., 2009; Lawless et al., 2017) individually across a broad frequency spectrum, their relative contributions have not been separated. It is further challenging to use previous results to decouple mechanisms because the test length scales of approximately $5-6 \mu \mathrm{m}$ for poroelasticity, which is locally determined, versus about $5 \mathrm{~mm}$ for intrinsic viscoelasticity, which is determined across the entire thickness, are polarized. As a consequence, the depth-dependent heterogeneous structure of cartilage, encompassing collagen direction and diameter, cannot be accurately benchmarked.

Dissipation caused by poroelasticity is length dependent, whereas dissipation caused by intrinsic viscoelasticity is not. This disparity permits us to discern the respective contributions of the two. The decoupled dissipation mechanisms have shown that intrinsic viscoelastic dissipation provides persistent wide-band dissipation at all length scales, while additional poroelastic dissipation raises the overall dissipation at small length scales. In order to model the poroelastic tissues, the poroelastic constitutive model has been developed in 1956 (Biot, 1956a; Biot, 1956b) and extended to accommodate oscillatory behavior (Cheng et al., 1991; Perrinez et al., 2009). A poroelastic material is characterized as a biphasic continuum consisting of a porous elastic matrix and a penetrating pore liquid. Volumetric deformation of the solid matrix causes fluid flow inside the material. Inversely, a liquid penetrated into the material effects a deformation of the matrix scaffold.

\subsection{Viscoelasticity in Wound-Healing}

Apart from the overall tissue inflammation, viscoelasticity can also occur on smaller length scales. For example, viscoelasticity itself can act as a characteristic indicator for the activation of leukocytes (Zak et al., 2021). The elastic and viscous properties of leukocytes evolve in parallel and have a well-defined proportion when measured dynamically using a micropipette
(Zak et al., 2021). Thus, viscoelasticity represents a mechanical specification that is intrinsically unique to each cell type. Using microfluidic system the flow behavior of red blood cells can be analyzed in a microcirculation-mimicking framework of PDMS microchannels that exhibit a channel width similar to cell size (Tomaiuolo et al., 2011). In a 3D microenvironment the immune cells can be embedded within the $3 \mathrm{D}$ matrix scaffold and survive longer without the excessive addition of growth factors. Subsequently, the viscoelastic characteristics of the immune cells are be maintained on longer time intervals and thus the wound-healing process can be modeled in a more physiological manner.

For proper tissue and wound repair, it is critical that the viscoelastic, physical, and biodegradable characteristics of the scaffolds can be precisely adjusted to optimize cell performance, such as cell attachment, proliferation, and infiltration, all of which are essential to the healing process (Sharma et al., 2016). For the latter process, the motility of the immune cells is of critical importance that relies on their capacity to transmigrate out of endothelial blood vessels and invade through the extracellular matrix toward the sites of inflammation/injury. Apart from immune cells, fibroblasts and organ-specific epithelial cells are recruited to the sites of tissue injury in order to close the wound. By release of matrix cross-linking or matrix degrading substances, these cell type can have an impact on the mechanical phenotype of matrices, which in turn alter the viscoelastic response of embedded cells, such as fibroblasts, epithelial cells and endothelial cells.

For example, reestablishment of lung homeostasis after wounding necessitates efficacious wound healing through the epithelium. Among the mechanisms of epithelial wound healing in the lung are the dissemination and migration of cells into the injured zone and thereafter cell proliferation. It can be hypothesized that the mechanical characteristics of cells vary proximal to the wound margin and therefore may yield evidence to guide cell migration (Wagh et al., 2008). In the case that the epithelium is injured, it is critical to promptly rebuild the barrier's intactness in order to avoid additional infection or deterioration. The normal repair mechanism of airway epithelial cells, which has been examined in cell cultures (Savla et al., 1997; Desai et al., 2004), in animals (Park et al., 2006), and finally in humans (Heguy et al., 2007), comprises a coordinated cascade of sequential episodes that involve formation of a temporary matrix, dedifferentiation of proximal secretory and ciliated cells, swift attenuation and spreading of these cells, immigration into the injured region to cap the surface, and, subsequently, proliferation and differentiation of cells to restore the functionality of the epithelium (Holgate, 2004; Hackett and Knight, 2007). The extremely early phases of epithelial restoration, which include cell spreading and migration, emerge within hours of wounding, and these events require significant tremendous reshaping of the cellular structure. Moreover, the remodeling event in epithelial cells entails the concerted locomotion of a layer of cells rather than the migration of single cells.

The dynamic rearrangement that proceeds during cell spreading and migration is based on the generation of 
directional polarity of migrating cells and the elongation of protrusions in the path of migration (Lauffenburger and Horwitz, 1996; Ridley, 2003; Kole et al., 2005). These protrusions can be in the shape of wider lamellipodial projections or extended and slender filopodia. This is closely followed by a repetitive sequence of processes in which the protrusions build adhesions with the substrate, posterior adhesions are broken down, and contractile mechanisms draw the cell anteriorly. An essential component of these events is the tightly managed reorganization of cytoskeletal structures at distinct locations inside the cell. Actin filament remodeling, adhesion site generation and turnover, and actomyosin contraction are governed in some part by members of the Rho family of small GTPases (Van Aelst, 2002; Holgate, 2004). Therefore, it has been physically revealed previously that an unbalance in the activity of RhoA or Racl can interfere with normal repair mechanisms in airway epithelial cells (Desai et al., 2004). Due to its salient involvement in spatially regulated actinmyosin contraction events, which are indispensable for cells to produce the forces essential to pull ahead in cell migration, either an increase or a decrease in RhoA activity induced alterations in actin partitioning and led to substantially slower epithelial damage repair.

It is possible to alter membrane tension by changing phospholipid composition or by osmotically swelling cells (Raucher and Sheetz, 2000). They found that the lamellipodial expansion rate decreased with increasing membrane tension. They hypothesized that membrane tension levels may govern the rate of actin polymerization throughout lamellipodial expansion. Relative to cells adhering to a rigid substrate, cells adhering to softer substrates display fewer organized actin reticulations and fainter focal adhesions, and produce fewer tension (Felsenfeld et al., 1999). It has been proven that polymerization of microtubules toward the adhesion sites augments under elevated stress when force is exerted on the cell body with a microneedle or when the substrate is uniaxially stretched (Kaverina et al., 2002). It has been consistently illustrated that mechanical stretch and compression events pronouncedly reduce the motility of cells and wound sealing within airway epithelial cells (Savla et al., 1997; Savla and Waters, 1998). Yet, there is limited knowledge about how locally mechanical characteristics change throughout cell migration (Rotsch et al., 1999; Nagayama et al., 2001; Kole et al., 2005; Laurent et al., 2005). Therefore, it can be hypothesized that local mechanical characteristics alter as respiratory epithelial cells migrate, perhaps either as a cue to somehow concert the cell migration or in reaction to structural reorganizations occurring within the cells.

The central part of a migrating fibroblast initially stiffened in comparison to the central part of a stalled cell, however, then the stiffness in the central part is pronouncedly reduced in the course of cell migration (Nagayama et al., 2001). When migrating and stalled fibroblasts are compared, intracellular mechanical properties have been revealed employing microinjected fluorescent microbeads (Kole et al., 2005). In fact, after $4 \mathrm{~h}$, the cells at the border of an injured cell layer are not so much deformable and hence stiffer than resting fibroblasts.

\subsection{Viscoelasticity in Fibrosis}

Viscoelasticity is a mechanical property of multiple cell types, cell spheroids, tissues and entire organs. Among the tissues, the liver and the lung have frequently been chosen as model systems, as the both organs are quite prone for inflammatory diseases, including fibrosis. Fibrosis, which is the development of scar tissue as a consequence of overly impaired wound healing, is associated with a substantial increment in the accumulation of extracellular matrix. Inflammation itself is known to be an essential driver of fibrosis. During the process of fibrosis, the motility of fibroblasts in particular is an important driving factor.

In vivo magnetic resonance elastography measurements have demonstrated that the prevalence of liver inflammation and fibrosis leads to increased stiffness values (Huwart et al., 2006; Sinkus et al., 2018). This alteration can also render embedded cells, such as fibroblasts more elastically and less viscoelastic, which in turn consequently elevates their migratory capacity. However, disease coexistence (Mueller, 2010) and major deformation error bias in elastography measurements (Capilnasiu et al., 2019) may confound diagnosis even more. In recent decades, a number of rheological assays have been performed to accurately define liver tissue, with uniaxial deformation (either by loading a small specimen or by indenting the entire organ) and shear being the most widely applied. Both oscillatory shear and uniaxial deformation response experiments indicate that the liver presents quasi-linearity at low strains, with nonlinear performance emerging at higher strains (Gao et al., 2010; Liu and Bilston, 2000; Tan et al., 2013). In addition, loading/unloading experiments show that hysteresis effects occur (Jordan et al., 2011), where the reaction is frequency sensitive (Liu and Bilston, 2000; Miller, 2000). Multifrequency soft tissue assessments of shear modulus $\mathrm{G}^{*}$ reveal a fractional order reliance on angular frequency (Sinkus et al., 2018). Other biomechanical features of the liver have also been studied, including relaxation (Chatelin et al., 2011; Liu and Bilston, 2002) and creep (Wang et al., 1992).

Measurements of liver tissue rheology have resulted in a number of biomechanical models. Hyperelasticity is frequently implied, with polynomial, exponential, and logarithmic shapes adopted for compression and strain measurements (Chui et al., 2004; Gao et al., 2010). The general results imply that the exponential, logarithmic, and power law models provide greater versatility in covering the various ranges of stressstrain curves. To study viscoelasticity, cyclic deformation or relaxation assays generally are required. Specific investigations utilized relaxation (Liu and Bilston, 2002), shear oscillations (Nicolle et al., 2010; Nicolle et al., 2013) or cyclic indentation probing (Jordan et al., 2011) over a broad spectrum of frequencies, to obtain a more comprehensive view of the biomechanical response of the liver. Of these models, the Kaye-Bernstein-Kearsley-Zapas model has been recommended because it takes into account the entire time history and has been confirmed for small-amplitude oscillatory shear and strain gradients (Nicolle et al., 2010, Nicolle et al., 2013). Optionally, viscoelasticity could be simulated through the implementation of a Maxwell element. A sophisticated differential model with ten different model parameters has been examined in terms of 
relaxation behavior at four strain levels (Liu and Bilston, 2002). A Maxwell-based model with 13 different variables has also been presented to evaluate the viscoelastic response of the liver under a variety of uniaxial preloads, frequencies, and strain rates (Ayyildiz et al., 2015). Large preloads (20\%) and shear strains (5\%) have been simultaneously utilized in the experimental protocol there. Nevertheless, the findings concentrate on the impacts of preload, strain rate, and frequency on the normal force and torque reaction (Tan et al., 2018). A comprehensive 3D model has been presented that can characterize the tissue reaction under different kinds of deformation and frequencies. Cross analysis of uniaxial preloads, such as $1-20 \%$, shear strains of $1-50 \%$ and frequencies ranging from $0.5-2 \mathrm{~Hz}$ has been proposed (Tan et al., 2013), highlighting the rate-dependent nonlinear viscoelastic characteristic of the liver. The measurement protocol exhibits a strain softening phenomenon, which is counteracted by suggesting a new error standard that permits a certain level of flexibility in adjusting the linear inputs to the models. This type of analysis and the process of model fitting results in the determination of simplified constitutive models that contain the key ingredients required to accurately represent the aforementioned characteristics of the liver subjected to both combinatory deformation and a variety of frequencies. Specifically, this is apparently among the first liver investigations to examine combined large uniaxial and shear stresses at multiple frequencies, and suggests a $3 \mathrm{D}$ nonlinear viscoelastic model with the ability to account for the largeamplitude oscillatory response over a wide array of preloads and frequencies (Capilnasiu et al., 2020).

For modelling, it is hypothesized that bovine liver specimen are stress free and isotropic, when they are in the reference configuration. The monitored torque characteristics are fit to a viscoelastic adjustment of three hyperelastic models typically employed in soft-tissue mechanics to compare their adequacy for sculpting the dataset. These are the polynomial model (a modified type of the Mooney-Rivlin model, which is referred to as $\mathrm{vMR}^{\star}$ ), which is the simplest model, the viscoelastic Ogden (vOG) model, which is based on the strain-energy function, and the viscoelastic exponential (vEXP) model, which is the isotropic exponential Fung-type model based on the strain energy function. The latter two models are similarly appropriate, as the two models are better at accounting for the nonlinear tendencies (Capilnasiu et al., 2020).

The mechanical features of lung parenchymal tissue represent elastic and dissipative elements, as well as they exhibit a highly nonlinear response. The mechanical response of lung tissue is based on a macroscopic phenomenon that is itself evoked by interactions of the microscopic elements. The mechanics of the pulmonary system are governed through the individual contributions of and the intricate interference among its major compounds. On the one hand, the lung tissues display a quasistatic mechanical behavior. On the other hand, there exist computational models that indicate how smooth nonlinear stress-strain response can emerge by a percolation-like event, where the sequential homing of collagen fibers with elevating strain renders them to progressively involved in performing the load-bearing function from elastin. The viscoelastic nature of lung tissue has been measured (de Hilster et al., 2019a). While the airway characteristics can be well presented through resistive and inertive properties (Hantos et al., 1992), the contribution of the lung tissues in the entire mechanical properties of the lung is more intricate, since there is a complex interference between the extravascular proteins, fibers, cells, surface film layer, and interstitial fluids (Bates et al., 2007; Faffe and Zin, 2009; Suki and Bates, 2011). The in vitro rebuilding of diseased and nondiseased states of the lung can be successfully performed employing hydrogels (de Hilster et al., 2019b).

Fibrosis and elevated stiffness are features of multiple solid cancers and encourage growth of the tumor. Cultivation of mammary epithelial cells on soft and rigid collagen matrices, respectively, reveals that with enhanced stiffness, epithelial tubulogenesis is reduced, contractility is augmented, and proliferation is amplified (Wozniak et al., 2003; Paszek et al., 2005; Provenzano et al., 2009). It has also been illustrated that growth in a more stiff ambient environment fosters an invasive phenotype (Provenzano et al., 2006). Many of these hallmarks are due to the enhanced activity of RhoA in cells when cultured in a rigid setting.

Attenuation of p190RhoGAP has been implicated as an additional mechanism for enhanced RhoA activity in settings of mechanical tension, such as in fibroblasts derived from patients with idiopathic pulmonary fibrosis (Monaghan-Benson et al., 2018). The etiology of idiopathic pulmonary fibrosis is unclear, but increased levels of TGF $\beta$ are an important contributor to fibrosis, and there is ample support for an influential role of RhoA in this disease and other kinds of fibrosis. Examination of the signaling mechanisms that increase RhoA activity revealed that p190RhoGAP activity has been suppressed in fibrotic fibroblasts and in reaction to TGF $\beta$ (Monaghan-Benson et al., 2018). Mechanism investigation identified that the expression of Rnd3/RhoE, an activator of p190RhoGAP, has been depressed through TGF $\beta$. A hallmark of fibrotic tissue is its enhanced stiffness, which results from the accumulation of surplus extracellular matrix. Increased stiffness augments RhoA activity (Wozniak et al., 2003) and GEF-H1 has been associated with it (Heck et al., 2012). Remarkably, both Rnd3 expression and p190RhoGAP activity are reduced in fibroblasts attached to rigid supports, indicating that this signaling route also accounts for increased RhoA activity in cells subjected to a rigid environment (Monaghan-Benson et al., 2018). Since TGF $\beta$ activity is also induced upon stimulation by elevated mechanical tension and growth on stiff media (Wipff et al., 2007), this points to a beneficial feedforward pathway engaging Rnd3 and p190RhoGAP.

\section{FINAL REMARKS AND FUTURE PERSPECTIVES}

The exploration of viscoelasticity is still a major issue and carries an enormous potential besides cell stiffness to serve as a reliable and possibly as a universal biomarker for cellular migration and invasion. It may also harbor the potential to be adaptable both the migration of individual cells and a collection of cells. In specific, 
the adaption of the viscoelastic characteristics of migratory cells through EMT-regulated adjustments of adherens junction seems to be critical for providing efficient collective migration through tissues that offer physically divergent structural and mechanical architectures. Moreover, it can be stated that viscoelasticity of the migratory microenvironment is crucial to induce cell migration, engineer a material that enables the efficient migration of cells, and/or to regulate migration through the process of durotaxis. Thereby, new advances strategies highlight possible mechanisms facilitating the transfer of mechanical cues into the cells, including the expression of traditional transcriptional controllers of EMT, and in turn their impact on the alteration of the viscoelastic phenotype of migrating cells and their local microenvironments. To understand these processes in a more precise manner the formation of hallmarks for the individual cell migration may be beneficial.

In addition, it has to be accounted for that molecular signal transduction causes remodeling events at various cellular length scales, such as plasma membrane, cytoskeleton, organelles and nuclear components, These cellular remodeling events alter the tissue including its viscoelastic characteristics, and this new viscoelasticity of the tissue environment can now act on a long-range timescale to impact cellular, molecular, and viscoelastic characteristics of a neighbor tissue, such as a mechano-molecular feedback circuit timing the various processes, including the development of tumor microenvironments. Since tissue interferences are seen at a chemical scale through the secretion of molecules, it would be promising to investigate the interaction of viscoelasticity and secreted molecules in the regulation of single and collective cell movements. The involvement of these types of mechanomolecular feedback interferences seem to be highly crucial in the advancement for the forming and engineering of organanalogous structures, such as organoids, in the field of mechanics-based cancer or inflammation research. Thereby, it

\section{REFERENCES}

Abidine, Y., Giannetti, A., Revilloud, J., Laurent, V. M., and Verdier, C. (2021). Viscoelastic Properties in Cancer: From Cells to Spheroids. Cells 10 (7), 1704. doi:10.3390/cells10071704

Acloque, H., Adams, M. S., Fishwick, K., Bronner-Fraser, M., and Nieto, M. A. (2009). Epithelial-mesenchymal Transitions: The Importance of Changing Cell State in Development and Disease. J. Clin. Invest. 119 (6), 1438-1449. doi:10. 1172/JCI38019

Aermes, C., Hayn, A., Fischer, T., and Mierke, C. T. (2021). Cell Mechanical Properties of Human Breast Carcinoma Cells Depend on Temperature. Scientific Rep. 11 (1), 10771. doi:10.1038/s41598-021-90173-y

Aermes, C., Hayn, A., Fischer, T., and Mierke, C. T. (2020). Environmentally Controlled Magnetic Nano-Tweezer for Living Cells and Extracellular Matrices. Scientific Rep. 10 (1), 13453. doi:10.1038/s41598-020-70428-w

Aggarwal, B. B., Danda, D., Gupta, S., and Gehlot, P. (2009). Models for Prevention and Treatment of Cancer: Problems vs Promises. Biochem. Pharmacol. 78 (9), 1083-1094. doi:10.1016/j.bcp.2009.05.027

Albrecht-Buehler, G. (1980). Autonomous Movements of Cytoplasmic Fragments. Proc. Natl. Acad. Sci. 77 (11), 6639-6643. doi:10.1073/pnas.77.11.6639

Alert, R., and Trepat, X. (2020). Physical Models of Collective Cell Migration. Annu. Rev. Condensed Matter Phys. 11 (1), 77-101. doi:10.1146/annurevconmatphys-031218-013516 is even crucial to combine the analysis of molecular elements, viscoelastic variables of cells and their local microenvironment (Poh et al., 2014; Wrighton and Kiessling, 2015; Mierke, 2019).

Consequently, viscoelasticity represents a general characteristic feature for the vast majority of biological constituents and the majority of cells and tissues that experience a single force or multiple mechanical forces. Finally, the requirement of multidisciplinary studies combining biophysical and biochemical variables seem to be critical to obtain a knowledge of growing intricate living biological systems.

For future studies focusing on viscoelasticity and other cell mechanical characteristics, the biophysical probing techniques need to be comparable and hence an experimental study on the comparison of different biophysical techniques is highly needed to further explore the phenomenon of viscoelasticity and to develop a road-map on how to conduct viscoelasticity measurements and in what type of microenvironment. Ultimately, the future perspective is the investigation of viscoelasticity on various length scales ranging from single molecules to entire tissues or on different time scales in order to fully probe viscoelasticity.

\section{AUTHOR CONTRIBUTIONS}

The author confirms being the sole contributor of this work and has approved it for publication.

\section{ACKNOWLEDGMENTS}

The author acknowledges support from the German Research Foundation (DFG) and Universität Leipzig within the program of Open Access Publishing, and Thomas M. L. Mierke for critical proof reading of the manuscript.

Allahyari, Z., Gholizadeh, S., Chung, H. H., Delgadillo, L. F., and Gaborski, T. R. (2020). Micropatterned Poly(ethylene Glycol) Islands Disrupt Endothelial Cell-Substrate Interactions Differently from Microporous Membranes. ACS Biomater. Sci. Eng. 6 (2), 959-968. doi:10.1021/acsbiomaterials.9b01584

Alvarado, J., Sheinman, M., Sharma, A., MacKintosh, F. C., and Koenderink, G. H. (2017). Force Percolation of Contractile Active Gels. Soft Matter 13 (34), 5624-5644. doi:10.1039/C7SM00834A

Amano, M., Ito, M., Kimura, K., Fukata, Y., Chihara, K., Nakano, T., et al. (1996). Phosphorylation and Activation of Myosin by Rho-Associated Kinase (RhoKinase). J. Biol. Chem. 271 (34), 20246-20249. doi:10.1074/jbc.271.34.20246

Amblard, F., Maggs, A. C., Yurke, B., Pargellis, A. N., and Leibler, S. (1996). Subdiffusion and Anomalous Local Viscoelasticity in Actin Networks. Phys. Rev. Lett. 77 (21), 4470-4473. doi:10.1103/PhysRevLett.77.4470

Angelini, T. E., Hannezo, E., Trepat, X., Marquez, M., Fredberg, J. J., and Weitz, D. A. (2011). Glass-like Dynamics of Collective Cell Migration. Proc. Natl. Acad. Sci. 108 (12), 4714-4719. doi:10.1073/pnas.1010059108

Ansieau, S., Collin, G., and Hill, L. (2014). EMT or EMT-Promoting Transcription Factors, where to Focus the Light? Front. Oncol. 4. doi:10.3389/fonc.2014.00353

Asnacios, A., and Hamant, O. (2012). The Mechanics behind Cell Polarity. Trends Cel Biol. 22 (11), 584-591. doi:10.1016/j.tcb.2012.08.005

Atherton, P., Lausecker, F., Carisey, A., Gilmore, A., Critchley, D., Barsukov, I., et al. (2020). Relief of Talin Autoinhibition Triggers a Force-independent Association with Vinculin. J. Cel Biol. 219 (1), e201903134. doi:10.1083/jcb. 201903134 
Austen, K., Ringer, P., Mehlich, A., Chrostek-Grashoff, A., Kluger, C., Klingner, C., et al. (2015). Extracellular Rigidity Sensing by Talin Isoform-specific Mechanical Linkages. Nat. Cel Biol. 17 (12), 1597-1606. doi: $10.1038 /$ ncb3268

Ayyildiz, M., Cinoglu, S., and Basdogan, C. (2015). Effect of normal Compression on the Shear Modulus of Soft Tissue in Rheological Measurements. J. Mech. Behav. Biomed. Mater. 49, 235-243. doi:10.1016/j.jmbbm.2015.05.011

Azioune, A., Carpi, N., Tseng, Q., Théry, M., and Piel, M. (2010). Protein Micropatterns. Methods Cel Biol. 97, 133-146. doi:10.1016/S0091-679X(10) 97008-8

Azioune, A., Storch, M., Bornens, M., Théry, M., and Piel, M. (2009). Simple and Rapid Process for Single Cell Micro-patterning. Lab. A Chip 9 (11), 1640. doi:10. $1039 / \mathrm{b} 821581 \mathrm{~m}$

Bajanca, F., Gouignard, N., Colle, C., Parsons, M., Mayor, R., and Theveneau, E. (2019). In Vivo topology Converts Competition for Cell-Matrix Adhesion into Directional Migration. Nat. Commun. 10 (1), 1518. doi:10.1038/s41467-01909548-5

Banigan, E. J., Stephens, A. D., and Marko, J. F. (2017). Mechanics and Buckling of Biopolymeric Shells and Cell Nuclei. Biophysical J. 113 (8), 1654-1663. doi:10. 1016/j.bpj.2017.08.034

Banks, H. T., Hu, S., and Kenz, Z. R. (2011). A Brief Review of Elasticity and Viscoelasticity for Solids. Adv. Appl. Mathematics Mech. 3 (1), 1-51. doi:10. 4208/aamm.10-m1030

Bao, G., and Suresh, S. (2003). Cell and Molecular Mechanics of Biological Materials. Nat. Mater. 2 (11), 715-725. doi:10.1038/nmat1001

Bao, L., Sachs, F., and Dahl, G. (2004). Connexins Are Mechanosensitive. Am. J. Physiology-Cell Physiol. 287 (5), C1389-C1395. doi:10.1152/ajpcell.00220. 2004

Barer, R. (1952). Interference Microscopy and Mass Determination. Nature 169 (4296), 366-367. doi:10.1038/169366b0

Barriga, E. H., Franze, K., Charras, G., and Mayor, R. (2018). Tissue Stiffening Coordinates Morphogenesis by Triggering Collective Cell Migration In Vivo. Nature 554 (7693), 523-527. doi:10.1038/nature25742

Barriga, E. H., and Mayor, R. (2019). Adjustable Viscoelasticity Allows for Efficient Collective Cell Migration. Semin. Cel Developmental Biol. 93, 55-68. doi:10. 1016/j.semcdb.2018.05.027

Barriga, E. H., and Mayor, R. (2015). Embryonic Cell-Cell Adhesion. Curr. Top. Developmental Biol. 112, 301-323. doi:10.1016/bs.ctdb.2014.11.023

Barriga, E. H., and Theveneau, E. (2020). In Vivo Neural Crest Cell Migration Is Controlled by "Mixotaxis. Front. Physiol. 11, 586432. doi:10.3389/fphys.2020. 586432

Batchelder, E. L., Hollopeter, G., Campillo, C., Mezanges, X., Jorgensen, E. M., Nassoy, P., et al. (2011). Membrane Tension Regulates Motility by Controlling Lamellipodium Organization. Proc. Natl. Acad. Sci. 108 (28), 11429-11434. doi:10.1073/pnas.1010481108

Bates, J. H. T., Davis, G. S., Majumdar, A., Butnor, K. J., and Suki, B. (2007). Linking Parenchymal Disease Progression to Changes in Lung Mechanical Function by Percolation. Am. J. Respir. Crit. Care Med. 176 (6), 617-623. doi:10.1164/rccm. 200611-1739OC

Baum, B., and Georgiou, M. (2011). Dynamics of Adherens Junctions in Epithelial Establishment, Maintenance, and Remodeling. J. Cel Biol. 192 (6), 907-917. doi:10.1083/jcb.201009141

Baumgarten, K., and Tighe, B. P. (2017). Viscous Forces and Bulk Viscoelasticity Near Jamming. Soft Matter 13 (45), 8368-8378. doi:10.1039/C7SM01619K

Bausch, A. R., Möller, W., and Sackmann, E. (1999). Measurement of Local Viscoelasticity and Forces in Living Cells by Magnetic Tweezers. Biophysical J. 76 (1), 573-579. doi:10.1016/S0006-3495(99)77225-5

Bausch, A. R., Ziemann, F., Boulbitch, A. A., Jacobson, K., and Sackmann, E. (1998). Local Measurements of Viscoelastic Parameters of Adherent Cell Surfaces by Magnetic Bead Microrheometry. Biophysical J. 75 (4), 2038-2049. doi:10.1016/S0006-3495(98)77646-5

Bazellières, E., Conte, V., Elosegui-Artola, A., Serra-Picamal, X., Bintanel-Morcillo, M., Roca-Cusachs, P., et al. (2015). Control of Cell-Cell Forces and Collective Cell Dynamics by the Intercellular Adhesome. Nat. Cel Biol. 17 (4), 409-420. doi:10.1038/ncb3135

Benedito, R., Roca, C., Sörensen, I., Adams, S., Gossler, A., Fruttiger, M., et al. (2009). The Notch Ligands Dll4 and Jagged1 Have Opposing Effects on Angiogenesis. Cell 137 (6), 1124-1135. doi:10.1016/j.cell.2009.03.025
Beningo, K. A., Lillie, S. H., and Brown, S. S. (2000). The Yeast Kinesin-Related Protein Smylp Exerts its Effects on the Class V Myosin Myo2p via a Physical Interaction. Mol. Biol. Cel 11 (2), 691-702. doi:10.1091/mbc.11.2.691

Benjamin, J. M., Kwiatkowski, A. V., Yang, C., Korobova, F., Pokutta, S., Svitkina, T., et al. (2010). Ae-catenin Regulates Actin Dynamics Independently of Cadherin-Mediated Cell-Cell Adhesion. J. Cel Biol. 189 (2), 339-352. doi:10. 1083/jcb.200910041

Berg, H. C. (1984). Random Walks in Biology. New and Expanded Edition. a. doi:10.1515/9781400820023

Berner, J., Müller, B., Gomez-Solano, J. R., Krüger, M., and Bechinger, C. (2018). Oscillating Modes of Driven Colloids in Overdamped Systems. Nat. Commun. 9 (1), 999. doi:10.1038/s41467-018-03345-2

Bersini, S., Jeon, J. S., Dubini, G., Arrigoni, C., Chung, S., Charest, J. L., et al. (2014). A Microfluidic 3D In Vitro Model for Specificity of Breast Cancer Metastasis to Bone. Biomaterials 35 (8), 2454-2461. doi:10.1016/j.biomaterials.2013.11.050

Bettinger, C. J., Langer, R., and Borenstein, J. T. (2009). Engineering Substrate Topography at the Micro- and Nanoscale to Control Cell Function. Angew. Chem. Int. Edition 48 (30), 5406-5415. doi:10.1002/anie.200805179

Bhatia, S. N., and Ingber, D. E. (2014). Microfluidic Organs-On-Chips. Nat. Biotechnol. 32 (8), 760-772. doi:10.1038/nbt.2989

Bi, D., Lopez, J. H., Schwarz, J. M., and Manning, M. L. (2015). A Densityindependent Rigidity Transition in Biological Tissues. Nat. Phys. 11 (12), 1074-1079. doi:10.1038/nphys3471

Bi, D., Yang, X., Marchetti, M. C., and Manning, M. L. (2016). Motility-Driven Glass and Jamming Transitions in Biological Tissues. Phys. Rev. X 6 (2), 021011. doi:10.1103/PhysRevX.6.021011

Bieling, P., Li, T.-D., Weichsel, J., McGorty, R., Jreij, P., Huang, B., et al. (2016). Force Feedback Controls Motor Activity and Mechanical Properties of SelfAssembling Branched Actin Networks. Cell 164 (1-2), 115-127. doi:10.1016/j. cell.2015.11.057

Biot, M. A. (1956b). Theory of Propagation of Elastic Waves in a Fluid-Saturated Porous Solid. II. Higher Frequency Range. The J. Acoust. Soc. America 28 (2), 179-191. doi:10.1121/1.1908241

Biot, M. A. (1956a). Theory of Propagation of Elastic Waves in a Fluid-Saturated Porous Solid. I. Low-Frequency Range. J. Acoust. Soc. America 28 (2), 168-178. doi:10.1121/1.1908239

Bonn, D., Denn, M. M., Berthier, L., Divoux, T., and Manneville, S. (2017). Yield Stress Materials in Soft Condensed Matter. Rev. Mod. Phys. 89 (3), 035005. doi:10.1103/RevModPhys.89.035005

Borghi, N., and Brochard-Wyart, F. (2007). Tether Extrusion from Red Blood Cells: Integral Proteins Unbinding from Cytoskeleton. Biophysical J. 93 (4), 1369-1379. doi:10.1529/biophysj.106.087908

Bosveld, F., Bonnet, I., Guirao, B., Tlili, S., Wang, Z., Petitalot, A., et al. (2012). Mechanical Control of Morphogenesis by Fat/Dachsous/Four-Jointed Planar Cell Polarity Pathway. Science 336 (6082), 724-727. doi:10.1126/science. 1221071

Box, K., Joyce, B. W., and Devenport, D. (2019). Epithelial Geometry Regulates Spindle Orientation and Progenitor Fate during Formation of the Mammalian Epidermis. ELife 8, e47102. doi:10.7554/eLife.47102

Brabletz, T., Kalluri, R., Nieto, M. A., and Weinberg, R. A. (2018). EMT in Cancer. Nat. Rev. Cancer 18 (2), 128-134. doi:10.1038/nrc.2017.118

Brighenti, R., and Vernerey, F. J. (2017). A Simple Statistical Approach to Model the Time-dependent Response of Polymers with Reversible Cross-Links. Composites B: Eng. 115, 257-265. doi:10.1016/j.compositesb.2016.09.090

Broedersz, C. P., and MacKintosh, F. C. (2014). Modeling Semiflexible Polymer Networks. Rev. Mod. Phys. 86 (3), 995-1036. doi:10.1103/RevModPhys.86.995

Broers, J. L. V., Peeters, E. A. G., Kuijpers, H. J. H., Endert, J., Bouten, C. V. C., Oomens, C. W. J., et al. (2004). Decreased Mechanical Stiffness in LMNA-/Cells Is Caused by Defective Nucleo-Cytoskeletal Integrity: Implications for the Development of Laminopathies. Hum. Mol. Genet. 13 (21), 2567-2580. doi:10. 1093/hmg/ddh295

Bröhl, D., Vasyutina, E., Czajkowski, M. T., Griger, J., Rassek, C., Rahn, H.-P., et al. (2012). Colonization of the Satellite Cell Niche by Skeletal Muscle Progenitor Cells Depends on Notch Signals. Developmental Cel 23 (3), 469-481. doi:10. 1016/j.devcel.2012.07.014

Bugaj, L. J., Choksi, A. T., Mesuda, C. K., Kane, R. S., and Schaffer, D. V. (2013). Optogenetic Protein Clustering and Signaling Activation in Mammalian Cells. Nat. Methods 10 (3), 249-252. doi:10.1038/nmeth.2360 
Burla, F., Mulla, Y., Vos, B. E., Aufderhorst-Roberts, A., and Koenderink, G. H. (2019). From Mechanical Resilience to Active Material Properties in Biopolymer Networks. Nat. Rev. Phys. 1 (4), 249-263. doi:10.1038/s42254019-0036-4

Burridge, K., Monaghan-Benson, E., and Graham, D. M. (2019). Mechanotransduction: From the Cell Surface to the Nucleus via RhoA. Philosophical Trans. R. Soc. B: Biol. Sci. 374 (1779), 20180229. doi:10.1098/ rstb.2018.0229

Burridge, K., Turner, C. E., and Romer, L. H. (1992). Tyrosine Phosphorylation of Paxillin and pp125FAK Accompanies Cell Adhesion to Extracellular Matrix: A Role in Cytoskeletal Assembly. J. Cel Biol. 119 (4), 893-903. doi:10.1083/jcb. 119.4.893

Burstein, A. H., and Frankel, V. H. (1968). The Viscoelastic Properties of Some Biological Materials. Ann. New York Acad. Sci. 146 (1), 158-165. doi:10.1111/j. 1749-6632.1968.tb20280.x

Buxboim, A., Rajagopal, K., Brown, A. E. X., and Discher, D. E. (2010). How Deeply Cells Feel: Methods for Thin Gels. J. Phys. Condensed Matter 22 (19), 194116. doi:10.1088/0953-8984/22/19/194116

Cai, D., Dai, W., Prasad, M., Luo, J., Gov, N. S., and Montell, D. J. (2016). Modeling and Analysis of Collective Cell Migration in an In Vivo Three-Dimensional Environment. Proc. Natl. Acad. Sci. 113 (15), E2134-E2141. doi:10.1073/pnas. 1522656113

Calero-Cuenca, F. J., Janota, C. S., and Gomes, E. R. (2018). Dealing with the Nucleus during Cell Migration. Curr. Opin. Cel Biol. 50, 35-41. doi:10.1016/j. ceb.2018.01.014

Calvo, F., Ege, N., Grande-Garcia, A., Hooper, S., Jenkins, R. P., Chaudhry, S. I., et al. (2013). Mechanotransduction and YAP-dependent Matrix Remodelling Is Required for the Generation and Maintenance of Cancer-Associated Fibroblasts. Nat. Cel Biol. 15 (6), 637-646. doi:10.1038/ncb2756

Camley, B. A., Zhang, Y., Zhao, Y., Li, B., Ben-Jacob, E., Levine, H., et al. (2014). Polarity Mechanisms Such as Contact Inhibition of Locomotion Regulate Persistent Rotational Motion of Mammalian Cells on Micropatterns. Proc. Natl. Acad. Sci. 111 (41), 14770-14775. doi:10.1073/pnas.1414498111

Campbell, K., and Casanova, J. (2016). A Common Framework for EMT and Collective Cell Migration. Development 143 (23), 4291-4300. doi:10.1242/dev. 139071

Canel, M., Serrels, A., Frame, M. C., and Brunton, V. G. (2013). E-cadherin-integrin Crosstalk in Cancer Invasion and Metastasis. J. Cel Sci. 126 (2), 393-401. doi:10.1242/jcs.100115

Caolo, V., Debant, M., Endesh, N., Futers, T. S., Lichtenstein, L., Bartoli, F., et al. (2020). Shear Stress Activates ADAM10 Sheddase to Regulate Notch1 via the Piezo1 Force Sensor in Endothelial Cells. ELife 9, e50684. doi:10.7554/eLife.50684

Capco, D. G., Wan, K. M., and Penman, S. (1982). The Nuclear Matrix: ThreeDimensional Architecture and Protein Composition. Cell 29 (3), 847-858. doi:10.1016/0092-8674(82)90446-9

Capilnasiu, A., Bilston, L., Sinkus, R., and Nordsletten, D. (2020). Nonlinear Viscoelastic Constitutive Model for Bovine Liver Tissue. Biomech. Model. Mechanobiology 19 (5), 1641-1662. doi:10.1007/s10237-020-01297-5

Capilnasiu, A., Hadjicharalambous, M., Fovargue, D., Patel, D., Holub, O., Bilston, L., et al. (2019). Magnetic Resonance Elastography in Nonlinear Viscoelastic Materials under Load. Biomech. Model. Mechanobiology 18 (1), 111-135. doi:10.1007/s10237-018-1072-1

Capuana, L., Boström, A., and Etienne-Manneville, S. (2020). Multicellular Scale Front-To-Rear Polarity in Collective Migration. Curr. Opin. Cel Biol. 62, 114-122. doi:10.1016/j.ceb.2019.10.001

Carlsson, A. E. (2010). Actin Dynamics: From Nanoscale to Microscale. Annu. Rev. Biophys. 39 (1), 91-110. doi:10.1146/annurev.biophys.093008.131207

Carmona-Fontaine, C., Theveneau, E., Tzekou, A., Tada, M., Woods, M., Page, K. M., et al. (2011). Complement Fragment C3a Controls Mutual Cell Attraction during Collective Cell Migration. Developmental Cel 21 (6), 1026-1037. doi:10. 1016/j.devcel.2011.10.012

Casillo, S. M., Peredo, A. P., Perry, S. J., Chung, H. H., and Gaborski, T. R. (2017). Membrane Pore Spacing Can Modulate Endothelial Cell-Substrate and Cell-Cell Interactions. ACS Biomater. Sci. Eng. 3 (3), 243-248. doi:10.1021/ acsbiomaterials.7b00055

Cavey, M., and Lecuit, T. (2009). Molecular Bases of Cell-Cell Junctions Stability and Dynamics. Cold Spring Harbor Perspect. Biol. 1 (5), a002998. doi:10.1101/ cshperspect.a002998
Ceballos, S., Kandel, M., Sridharan, S., Majeed, H., Monroy, F., and Popescu, G. (2015). Active Intracellular Transport in Metastatic Cells Studied by Spatial Light Interference Microscopy. J. Biomed. Opt. 20 (11), 111209. doi:10.1117/1. JBO.20.11.111209

Chan, C. J., Ekpenyong, A. E., Golfier, S., Li, W., Chalut, K. J., Otto, O., et al. (2015). Myosin II Activity Softens Cells in Suspension. Biophysical J. 108 (8), 1856-1869. doi:10.1016/j.bpj.2015.03.009

Charras, G., and Sahai, E. (2014). Physical Influences of the Extracellular Environment on Cell Migration. Nat. Rev. Mol. Cel Biol. 15 (12), 813-824. doi:10.1038/nrm3897

Charras, G., and Yap, A. S. (2018). Tensile Forces and Mechanotransduction at Cell-Cell Junctions. Curr. Biol. 28 (8), R445-R457. doi:10.1016/j.cub.2018. 02.003

Charrier, E. E., and Janmey, P. A. (2016). Mechanical Properties of Intermediate Filament Proteins. Methods Enzymol. 568, 35-57. doi:10.1016/bs.mie.2015. 09.009

Chatelin, S., Oudry, J., Périchon, N., Sandrin, L., Allemann, P., Soler, L., et al. (2011). In Vivo liver Tissue Mechanical Properties by Transient Elastography: Comparison with Dynamic Mechanical Analysis. Biorheology 48 (2), 75-88. doi:10.3233/BIR-2011-0584

Chaudhuri, O., Cooper-White, J., Janmey, P. A., Mooney, D. J., and Shenoy, V. B. (2020). Effects of Extracellular Matrix Viscoelasticity on Cellular Behaviour. Nature 584 (7822), 535-546. doi:10.1038/s41586-020-2612-2

Che, B., Zhao, W., Liu, Y., Sun, D., Jing, G., Bai, J., et al. (2021). Dynamic Intracellular Mechanical Cues Facilitate Collective Signaling Responses. IScience 24 (5), 102396. doi:10.1016/j.isci.2021.102396

Chen, T., Saw, T. B., Mège, R.-M., and Ladoux, B. (2018). Mechanical Forces in Cell Monolayers. J. Cel Sci. 131 (24), jcs218156. doi:10.1242/jcs.218156

Cheng, A. H. -D., Badmus, T., and Beskos, D. E. (1991). Integral Equation for Dynamic Poroelasticity in Frequency Domain with BEM Solution. J. Eng. Mech. 117 (5), 1136-1157. doi:10.1061/(ASCE)0733-9399

Cho, S., Irianto, J., and Discher, D. E. (2017). Mechanosensing by the Nucleus: From Pathways to Scaling Relationships. J. Cel Biol. 216 (2), 305-315. doi:10. $1083 /$ jcb. 201610042

Chowdhury, F., Li, I. T. S., Ngo, T. T. M., Leslie, B. J., Kim, B. C., Sokoloski, J. E., et al. (2016). Defining Single Molecular Forces Required for Notch Activation Using Nano Yoyo. Nano Lett. 16 (6), 3892-3897. doi:10.1021/acs.nanolett. 6b01403

Chrzanowska-Wodnicka, M., and Burridge, K. (1996). Rho-stimulated Contractility Drives the Formation of Stress Fibers and Focal Adhesions. J. Cel Biol. 133 (6), 1403-1415. doi:10.1083/jcb.133.6.1403

Chui, C., Kobayashi, E., Chen, X., Hisada, T., and Sakuma, I. (2004). Combined Compression and Elongation Experiments and Non-linear Modelling of Liver Tissue for Surgical Simulation. Med. Biol. Eng. Comput. 42 (6), 787-798. doi:10. 1007/BF02345212

Chung, H. H., Mireles, M., Kwarta, B. J., and Gaborski, T. R. (2018). Use of Porous Membranes in Tissue Barrier and Co-culture Models. Lab. A Chip 18 (12), 1671-1689. doi:10.1039/C7LC01248A

Clément, R., Dehapiot, B., Collinet, C., Lecuit, T., and Lenne, P.-F. (2017). Viscoelastic Dissipation Stabilizes Cell Shape Changes during Tissue Morphogenesis. Curr. Biol. 27 (20), 3132-3142. doi:10.1016/j.cub.2017.09.005

Cohen, D. J., James Nelson, W., and Maharbiz, M. M. (2014). Galvanotactic Control of Collective Cell Migration in Epithelial Monolayers. Nat. Mater. 13 (4), 409-417. doi:10.1038/nmat3891

Collins, C., Guilluy, C., Welch, C., O’Brien, E. T., Hahn, K., Superfine, R., et al. (2012). Localized Tensional Forces on PECAM-1 Elicit a Global Mechanotransduction Response via the Integrin-RhoA Pathway. Curr. Biol. 22 (22), 2087-2094. doi:10.1016/j.cub.2012.08.051

Contu, F., Rangel-Pozzo, A., Trokajlo, P., Wark, L., Klewes, L., Johnson, N., et al. (2018). Distinct 3D Structural Patterns of Lamin A/C Expression in Hodgkin and Reed-Sternberg Cells. Cancers 10 (9), 286. doi:10.3390/cancers10090286

Coon, B. G., Baeyens, N., Han, J., Budatha, M., Ross, T. D., Fang, J. S., et al. (2015). Intramembrane Binding of VE-Cadherin to VEGFR2 and VEGFR3 Assembles the Endothelial Mechanosensory Complex. J. Cel Biol. 208 (7), 975-986. doi:10. 1083/jcb.201408103

Copos, C., and Mogilner, A. (2020). A Hybrid Stochastic-Deterministic Mechanochemical Model of Cell Polarization. Mol. Biol. Cel 31 (15), 1637-1649. doi:10.1091/mbc.E19-09-0549 
Crick, F. H. C., and Hughes, A. F. W. (1950). The Physical Properties of Cytoplasm. Exp. Cel Res. 1 (1), 37-80. doi:10.1016/0014-4827(50)90048-6

Crisp, M., Liu, Q., Roux, K., Rattner, J. B., Shanahan, C., Burke, B., et al. (2006). Coupling of the Nucleus and Cytoplasm: Role of the LINC Complex. J. Cel Biol. 172 (1), 41-53. doi:10.1083/jcb.200509124

Crocker, J. C., and Hoffman, B. D. (2007). Multiple-Particle Tracking and TwoPoint Microrheology in Cells. Methods Cel Biol. 83, 141-178. doi:10.1016/ S0091-679X(07)83007-X

Cross, S. E., Jin, Y.-S., Rao, J., and Gimzewski, J. K. (2007). Nanomechanical Analysis of Cells from Cancer Patients. Nat. Nanotechnology 2 (12), 780-783. doi:10.1038/nnano.2007.388

Csucs, G., Michel, R., Lussi, J. W., Textor, M., and Danuser, G. (2003). Microcontact Printing of Novel Co-polymers in Combination with Proteins for Cell-Biological Applications. Biomaterials 24 (10), 1713-1720. doi:10.1016/ S0142-9612(02)00568-9

Cui, Y., and Bustamante, C. (2000). Pulling a Single Chromatin Fiber Reveals the Forces that Maintain its Higher-Order Structure. Proc. Natl. Acad. Sci. 97 (1), 127-132. doi:10.1073/pnas.97.1.127

Cupesi, M., Yoshioka, J., Gannon, J., Kudinova, A., Stewart, C. L., and Lammerding, J. (2010). Attenuated Hypertrophic Response to Pressure Overload in a Lamin A/C Haploinsufficiency Mouse. J. Mol. Cell Cardiol. 48 (6), 1290-1297. doi:10. 1016/j.yjmcc.2009.10.024

Czirók, A., Varga, K., Méhes, E., and Szabó, A. (2013). Collective Cell Streams in Epithelial Monolayers Depend on Cell Adhesion. New J. Phys. 15 (7), 075006. doi:10.1088/1367-2630/15/7/075006

Dahl, K. N., Engler, A. J., Pajerowski, J. D., and Discher, D. E. (2005). Power-Law Rheology of Isolated Nuclei with Deformation Mapping of Nuclear Substructures. Biophysical J. 89 (4), 2855-2864. doi:10.1529/biophysj.105.062554

Dahl, K. N., Kahn, S. M., Wilson, K. L., and Discher, D. E. (2004). The Nuclear Envelope Lamina Network Has Elasticity and a Compressibility Limit Suggestive of a Molecular Shock Absorber. J. Cel Sci. 117 (20), 4779-4786. doi: $10.1242 /$ jcs. 01357

Dai, W., Xiao, Y., Tang, W., Li, J., Hong, L., Zhang, J., et al. (2021). Identification of an EMT-Related Gene Signature for Predicting Overall Survival in Gastric Cancer. Front. Genet. 12, 661306. doi:10.3389/fgene.2021.661306

Dang, I., Gorelik, R., Sousa-Blin, C., Derivery, E., Guérin, C., Linkner, J., et al. (2013). Inhibitory Signalling to the Arp2/3 Complex Steers Cell Migration. Nature 503 (7475), 281-284. doi:10.1038/nature12611

D’Angelo, A., Dierkes, K., Carolis, C., Salbreux, G., and Solon, J. (2019). In Vivo Force Application Reveals a Fast Tissue Softening and External Friction Increase during Early Embryogenesis. Curr. Biol. 29 (9), 1564-1571. doi:10. 1016/j.cub.2019.04.010

Darling, E. M., Topel, M., Zauscher, S., Vail, T. P., and Guilak, F. (2008). Viscoelastic Properties of Human Mesenchymally-Derived Stem Cells and Primary Osteoblasts, Chondrocytes, and Adipocytes. J. Biomech. 41 (2), 454-464. doi:10.1016/j.jbiomech.2007.06.019

Davidson, P. M., and Lammerding, J. (2014). Broken Nuclei - Lamins, Nuclear Mechanics, and Disease. Trends Cel Biol. 24 (4), 247-256. doi:10.1016/j.tcb. 2013.11.004

Davies, H. G., and Wilkins, M. H. F. (1952). Interference Microscopy and Mass Determination. Nature 169 (4300), 541. doi:10.1038/169541a0

de Hilster, R. H. J., Jonker, M. R., Timens, W., Sharma, P. K., Harmsen, M. C., Burgess, J. K., et al. (2019a). Stiffness and Viscoelasticity of Human Lung Tissue Unaltered by Freeze-Thawing. Mech. Lung Inj. Repair, PA586. doi:10.1183/ 13993003.congress-2019.PA586

de Hilster, R. H. J., Jonker, M. R., Timens, W., Sharma, P. K., Harmsen, M. C., Hylkema, M. N., et al. (2019b). Human Lung Stiffness and Viscoelasticity Replicated in Extracellular Matrix Hydrogels. Mech. Lung Inj. Repair PA585. doi:10.1183/13993003.congress-2019.PA585

De La Cruz, E. M., and Gardel, M. L. (2015). Actin Mechanics and Fragmentation. J. Biol. Chem. 290 (28), 17137-17144. doi:10.1074/jbc.R115.636472

Deisseroth, K. (2011). Optogenetics. Nat. Methods 8 (1), 26-29. doi:10.1038/ nmeth.f.324

Deitch, S., Gao, B. Z., and Dean, D. (2012). Effect of Matrix on Cardiomyocyte Viscoelastic Properties in 2D Culture. Mol. Cell Biomech. MCB 9 (3), 227-249.

del Rio, A., Perez-Jimenez, R., Liu, R., Roca-Cusachs, P., Fernandez, J. M., and Sheetz, M. P. (2009). Stretching Single Talin Rod Molecules Activates Vinculin Binding. Science 323 (5914), 638-641. doi:10.1126/science.1162912
Delarue, M., Montel, F., Caen, O., Elgeti, J., Siaugue, J.-M., Vignjevic, D., et al. (2013). Mechanical Control of Cell Flow in Multicellular Spheroids. Phys. Rev. Lett. 110 (13), 138103. doi:10.1103/PhysRevLett.110.138103

Delarue, M., Montel, F., Vignjevic, D., Prost, J., Joanny, J.-F., and Cappello, G. (2014). Compressive Stress Inhibits Proliferation in Tumor Spheroids through a Volume Limitation. Biophysical J. 107 (8), 1821-1828. doi:10.1016/j.bpj.2014. 08.031

Denais, C., and Lammerding, J. (2014). "Nuclear Mechanics in Cancer,". Cancer Biology and the Nuclear Envelope. Editors E. C. Schirmer and J. I. de las Heras (New York: Springer), 773, 435-470. doi:10.1007/978-1-4899-8032-8_20

Denais, C. M., Gilbert, R. M., Isermann, P., McGregor, A. L., te Lindert, M., Weigelin, B., et al. (2016). Nuclear Envelope Rupture and Repair during Cancer Cell Migration. Science 352 (6283), 353-358. doi:10.1126/science.aad7297

Desai, L. P., Aryal, A. M., Ceacareanu, B., Hassid, A., and Waters, C. M. (2004). RhoA and Racl Are Both Required for Efficient Wound Closure of Airway Epithelial Cells. Am. J. Physiology-Lung Cell Mol. Physiol. 287 (6), L1134-L1144. doi:10.1152/ajplung.00022.2004

Desprat, N., Richert, A., Simeon, J., and Asnacios, A. (2005). Creep Function of a Single Living Cell. Biophysical J. 88 (3), 2224-2233. doi:10.1529/biophysj.104. 050278

Desprat, N., Supatto, W., Pouille, P.-A., Beaurepaire, E., and Farge, E. (2008). Tissue Deformation Modulates Twist Expression to Determine Anterior Midgut Differentiation in Drosophila Embryos. Developmental Cel 15 (3), 470-477. doi:10.1016/j.devcel.2008.07.009

Discher, D. E. (2005). Tissue Cells Feel and Respond to the Stiffness of Their Substrate. Science 310 (5751), 1139-1143. doi:10.1126/science.1116995

Diz-Muñoz, A., Thurley, K., Chintamen, S., Altschuler, S. J., Wu, L. F., Fletcher, D. A., et al. (2016). Membrane Tension Acts through PLD2 and mTORC2 to Limit Actin Network Assembly during Neutrophil Migration. PLOS Biol. 14 (6), e1002474. doi:10.1371/journal.pbio.1002474

Dolega, M. E., Monnier, S., Brunel, B., Joanny, J.-F., Recho, P., and Cappello, G. (2021). Extracellular Matrix in Multicellular Aggregates Acts as a Pressure Sensor Controlling Cell Proliferation and Motility. ELife 10, e63258. doi:10. 7554/eLife. 63258

Downing, T. L., Soto, J., Morez, C., Houssin, T., Fritz, A., Yuan, F., et al. (2013). Biophysical Regulation of Epigenetic State and Cell Reprogramming. Nat. Mater. 12 (12), 1154-1162. doi:10.1038/nmat3777

Doxzen, K., Vedula, S. R. K., Leong, M. C., Hirata, H., Gov, N. S., Kabla, A. J., et al. (2013). Guidance of Collective Cell Migration by Substrate Geometry. Integr. Biol. 5 (8), 1026. doi:10.1039/c3ib40054a

Driscoll, M. M., Chen, B. G., Beuman, T. H., Ulrich, S., Nagel, S. R., and Vitelli, V. (2016). The Role of Rigidity in Controlling Material Failure. Proc. Natl. Acad. Sci. 113 (39), 10813-10817. doi:10.1073/pnas.1501169113

Driscoll, T. P., Ahn, S. J., Huang, B., Kumar, A., and Schwartz, M. A. (2020). Actin Flow-dependent and -independent Force Transmission through Integrins. Proc. Natl. Acad. Sci. 117 (51), 32413-32422. doi:10.1073/pnas.2010292117

Dupont, S., Morsut, L., Aragona, M., Enzo, E., Giulitti, S., Cordenonsi, M., et al. (2011). Role of YAP/TAZ in Mechanotransduction. Nature 474 (7350), 179-183. doi:10.1038/nature10137

Efremov, Y. M., Wang, W.-H., Hardy, S. D., Geahlen, R. L., and Raman, A. (2017). Measuring Nanoscale Viscoelastic Parameters of Cells Directly from AFM Force-Displacement Curves. Scientific Rep. 7 (1), 1541. doi:10.1038/s41598017-01784-3

Ehrlicher, A. J., Nakamura, F., Hartwig, J. H., Weitz, D. A., and Stossel, T. P. (2011). Mechanical Strain in Actin Networks Regulates FilGAP and Integrin Binding to Filamin A. Nature 478 (7368), 260-263. doi:10.1038/nature10430

Ekpenyong, A. E., Whyte, G., Chalut, K., Pagliara, S., Lautenschläger, F., Fiddler, C., et al. (2012). Viscoelastic Properties of Differentiating Blood Cells Are Fate- and Function-dependent. PLoS ONE 7 (9), e45237. doi:10.1371/journal.pone. 0045237

Eldridge, W. J., Steelman, Z. A., Loomis, B., and Wax, A. (2017). Optical Phase Measurements of Disorder Strength Link Microstructure to Cell Stiffness. Biophysical J. 112 (4), 692-702. doi:10.1016/j.bpj.2016.12.016

Elosegui-Artola, A., Andreu, I., Beedle, A. E. M., Lezamiz, A., Uroz, M., Kosmalska, A. J., et al. (2017). Force Triggers YAP Nuclear Entry by Regulating Transport across Nuclear Pores. Cell 171 (6), 1397-1410. e14. doi:10.1016/j.cell.2017.10.008

Elosegui-Artola, A., Oria, R., Chen, Y., Kosmalska, A., Pérez-González, C., Castro, N., et al. (2016). Mechanical Regulation of a Molecular Clutch Defines Force 
Transmission and Transduction in Response to Matrix Rigidity. Nat. Cel Biol. 18 (5), 540-548. doi:10.1038/ncb3336

Elosegui-Artola, A. (2021). The Extracellular Matrix Viscoelasticity as a Regulator of Cell and Tissue Dynamics. Curr. Opin. Cel Biol. 72, 10-18. doi:10.1016/j.ceb. 2021.04.002

Elosegui-Artola, A., Trepat, X., and Roca-Cusachs, P. (2018). Control of Mechanotransduction by Molecular Clutch Dynamics. Trends Cel Biol. 28 (5), 356-367. doi:10.1016/j.tcb.2018.01.008

Engler, A. J., Humbert, P. O., Wehrle-Haller, B., and Weaver, V. M. (2009). Multiscale Modeling of Form and Function. Science 324 (5924), 208-212. doi:10.1126/science.1170107

Engler, A. J., Sen, S., Sweeney, H. L., and Discher, D. E. (2006). Matrix Elasticity Directs Stem Cell Lineage Specification. Cell 126 (4), 677-689. doi:10.1016/j. cell.2006.06.044

Esmaeili Pourfarhangi, K., Cardenas De La Hoz, E., Cohen, A. R., and Gligorijevic, B. (2018). Contact Guidance Is Cell Cycle-dependent. APL Bioeng. 2 (3), 031904. doi:10.1063/1.5026419

Esteves de Lima, J., Bonnin, M.-A., Birchmeier, C., and Duprez, D. (2016). Muscle Contraction Is Required to Maintain the Pool of Muscle Progenitors via YAP and NOTCH during Fetal Myogenesis. ELife 5, e15593. doi:10.7554/eLife.15593

Etienne-Manneville, S. (2011). Control of Polarized Cell Morphology and Motility by Adherens Junctions. Semin. Cel Developmental Biol. 22 (8), 850-857. doi:10. 1016/j.semcdb.2011.07.023

Ewald, A. J., Brenot, A., Duong, M., Chan, B. S., and Werb, Z. (2008). Collective Epithelial Migration and Cell Rearrangements Drive Mammary Branching Morphogenesis. Developmental Cel 14 (4), 570-581. doi:10.1016/j.devcel.2008. 03.003

Eze, M. O. (1992). Membrane Fluidity, Reactive Oxygen Species, and CellMediated Immunity: Implications in Nutrition and Disease. Med. Hypotheses 37 (4), 220-224. doi:10.1016/0306-9877(92)90191-E

Faffe, D. S., and Zin, W. A. (2009). Lung Parenchymal Mechanics in Health and Disease. Physiol. Rev. 89 (3), 759-775. doi:10.1152/physrev.00019.2007

Falconnet, D., Pasqui, D., Park, S., Eckert, R., Schift, H., Gobrecht, J., et al. (2004). A Novel Approach to Produce Protein Nanopatterns by Combining Nanoimprint Lithography and Molecular Self-Assembly. Nano Lett. 4 (10), 1909-1914. doi:10.1021/nl0489438

Fang, J. S., Coon, B. G., Gillis, N., Chen, Z., Qiu, J., Chittenden, T. W., et al. (2017). Shear-induced Notch-Cx37-P27 axis Arrests Endothelial Cell Cycle to Enable Arterial Specification. Nat. Commun. 8 (1), 2149. doi:10.1038/s41467-017-01742-7

Farge, E. (2003). Mechanical Induction of Twist in the Drosophila Foregut/ Stomodeal Primordium. Curr. Biol. 13 (16), 1365-1377. doi:10.1016/S09609822(03)00576-1

Farhadifar, R., Röper, J.-C., Aigouy, B., Eaton, S., and Jülicher, F. (2007). The Influence of Cell Mechanics, Cell-Cell Interactions, and Proliferation on Epithelial Packing. Curr. Biol. 17 (24), 2095-2104. doi:10.1016/j.cub.2007.11.049

Fedorchak, G. R., Kaminski, A., and Lammerding, J. (2014). Cellular Mechanosensing: Getting to the Nucleus of it All. Prog. Biophys. Mol. Biol. 115 (2-3), 76-92. doi:10.1016/j.pbiomolbio.2014.06.009

Felsenfeld, D. P., Schwartzberg, P. L., Venegas, A., Tse, R., and Sheetz, M. P. (1999). Selective Regulation of Integrin-Cytoskeleton Interactions by the Tyrosine Kinase Src. Nat. Cel Biol. 1 (4), 200-206. doi:10.1038/12021

Felsenthal, N., and Zelzer, E. (2017). Mechanical Regulation of Musculoskeletal System Development. Development 144 (23), 4271-4283. doi:10.1242/dev.151266

Fischer, T., Hayn, A., and Mierke, C. T. (2020). Effect of Nuclear Stiffness on Cell Mechanics and Migration of Human Breast Cancer Cells. Front. Cel Developmental Biol. 8, 393. doi:10.3389/fcell.2020.00393

Fischer, T., Wilharm, N., Hayn, A., and Mierke, C. T. (2017). Matrix and Cellular Mechanical Properties Are the Driving Factors for Facilitating Human Cancer Cell Motility into 3D Engineered Matrices. Convergent Sci. Phys. Oncol. 3 (4), 044003. doi:10.1088/2057-1739/aa8bbb

Fletcher, D. A., and Mullins, R. D. (2010). Cell Mechanics and the Cytoskeleton. Nature 463 (7280), 485-492. doi:10.1038/nature08908

Folker, E. S., Östlund, C., Luxton, G. W. G., Worman, H. J., and Gundersen, G. G. (2011). Lamin A Variants that Cause Striated Muscle Disease Are Defective in Anchoring Transmembrane Actin-Associated Nuclear Lines for Nuclear Movement. Proc. Natl. Acad. Sci. 108 (1), 131-136. doi:10.1073/pnas. 1000824108
Forgacs, G., Foty, R. A., Shafrir, Y., and Steinberg, M. S. (1998). Viscoelastic Properties of Living Embryonic Tissues: A Quantitative Study. Biophysical J. 74 (5), 2227-2234. doi:10.1016/S0006-3495(98)77932-9

Fouchard, J., Mitrossilis, D., and Asnacios, A. (2011). Acto-myosin Based Response to Stiffness and Rigidity Sensing. Cell Adhes. Migration 5 (1), 16-19. doi:10. 4161/cam.5.1.13281

Frangogiannis, N. G. (2016). Fibroblast-Extracellular Matrix Interactions in Tissue Fibrosis. Curr. Pathobiology Rep. 4 (1), 11-18. doi:10.1007/s40139016-0099-1

Friedl, P., and Gilmour, D. (2009). Collective Cell Migration in Morphogenesis, Regeneration and Cancer. Nat. Rev. Mol. Cel Biol. 10 (7), 445-457. doi:10.1038/ nrm2720

Friedl, P., Locker, J., Sahai, E., and Segall, J. E. (2012). Classifying Collective Cancer Cell Invasion. Nat. Cel Biol. 14 (8), 777-783. doi:10.1038/ncb2548

Friedl, P., and Weigelin, B. (2008). Interstitial Leukocyte Migration and Immune Function. Nat. Immunol. 9 (9), 960-969. doi:10.1038/ni.f.212

Friedl, P., Wolf, K., and Lammerding, J. (2011). Nuclear Mechanics during Cell Migration. Curr. Opin. Cel Biol. 23 (1), 55-64. doi:10.1016/j.ceb.2010.10.015

Friedl, P., and Wolf, K. (2010). Plasticity of Cell Migration: A Multiscale Tuning Model. J. Cel Biol. 188 (1), 11-19. doi:10.1083/jcb.200909003

Friedland, J. C., Lee, M. H., and Boettiger, D. (2009). Mechanically Activated Integrin Switch Controls $\alpha_{5} \beta_{1}$ Function. Science 323 (5914), 642-644. doi:10. 1126/science.1168441

Fuhrmann, A., Banisadr, A., Beri, P., Tlsty, T. D., and Engler, A. J. (2017). Metastatic State of Cancer Cells May Be Indicated by Adhesion Strength. Biophysical J. 112 (4), 736-745. doi:10.1016/j.bpj.2016.12.038

Fuhrmann, A., Staunton, J. R., Nandakumar, V., Banyai, N., Davies, P. C. W., and Ros, R. (2011). AFM Stiffness Nanotomography of normal, Metaplastic and Dysplastic Human Esophageal Cells. Phys. Biol. 8 (1), 015007. doi:10.1088/ 1478-3975/8/1/015007

Fulcher, G. R., Hukins, D. W., and Shepherd, D. E. (2009). Viscoelastic Properties of Bovine Articular Cartilage Attached to Subchondral Bone at High Frequencies. BMC Musculoskelet. Disord. 10 (1), 61. doi:10.1186/1471-2474-10-61

Gao, Z., Lister, K., and Desai, J. P. (2010). Constitutive Modeling of Liver Tissue: Experiment and Theory. Ann. Biomed. Eng. 38 (2), 505-516. doi:10.1007/ s10439-009-9812-0

Garcia, S., Hannezo, E., Elgeti, J., Joanny, J.-F., Silberzan, P., and Gov, N. S. (2015). Physics of Active Jamming during Collective Cellular Motion in a Monolayer. Proc. Natl. Acad. Sci. 112 (50), 15314-15319. doi:10.1073/pnas.1510973112

Gardel, M. L., Nakamura, F., Hartwig, J., Crocker, J. C., Stossel, T. P., and Weitz, D. A. (2006a). Stress-Dependent Elasticity of Composite Actin Networks as a Model for Cell Behavior. Phys. Rev. Lett. 96 (8), 088102. doi:10.1103/ PhysRevLett.96.088102

Gardel, M. L., Nakamura, F., Hartwig, J. H., Crocker, J. C., Stossel, T. P., and Weitz, D. A. (2006b). Prestressed F-Actin Networks Cross-Linked by Hinged Filamins Replicate Mechanical Properties of Cells. Proc. Natl. Acad. Sci. 103 (6), 1762-1767. doi:10.1073/pnas.0504777103

Gardel, M. L., Shin, J. H., MacKintosh, F. C., Mahadevan, L., Matsudaira, P., and Weitz, D. A. (2004). Elastic Behavior of Cross-Linked and Bundled Actin Networks. Science 304 (5675), 1301-1305. doi:10.1126/science.1095087

Gauthier, N. C., and Roca-Cusachs, P. (2018). Mechanosensing at IntegrinMediated Cell-Matrix Adhesions: From Molecular to Integrated Mechanisms. Curr. Opin. Cel Biol. 50, 20-26. doi:10.1016/i.ceb.2017.12.014

Gavara, N. (2016). Combined Strategies for Optimal Detection of the Contact point in AFM Force-Indentation Curves Obtained on Thin Samples and Adherent Cells. Scientific Rep. 6 (1), 21267. doi:10.1038/srep21267

Gavara, N., Roca-Cusachs, P., Sunyer, R., Farré, R., and Navajas, D. (2008). Mapping Cell-Matrix Stresses during Stretch Reveals Inelastic Reorganization of the Cytoskeleton. Biophysical J. 95 (1), 464-471. doi:10. 1529/biophysj.107.124180

Gavert, N., Sheffer, M., Raveh, S., Spaderna, S., Shtutman, M., Brabletz, T., et al. (2007). Expression of L1-CAM and ADAM10 in Human Colon Cancer Cells Induces Metastasis. Cancer Res. 67 (16), 7703-7712. doi:10.1158/0008-5472. CAN-07-0991

Geiger, B., and Yamada, K. M. (2011). Molecular Architecture and Function of Matrix Adhesions. Cold Spring Harbor Perspect. Biol., 3(5), a005033. doi:10. 1101/cshperspect.a005033 
Ghassemi, S., Meacci, G., Liu, S., Gondarenko, A. A., Mathur, A., Roca-Cusachs, P., et al. (2012). Cells Test Substrate Rigidity by Local Contractions on Submicrometer Pillars. Proc. Natl. Acad. Sci. 109 (14), 5328-5333. doi:10. 1073/pnas.1119886109

Gheldof, A., and Berx, G. (2013). Cadherins and Epithelial-To-Mesenchymal Transition. Prog. Mol. Biol. Translational Sci. 116, 317-336. doi:10.1016/ B978-0-12-394311-8.00014-5

Gibson, C. T., Smith, D. A., and Roberts, C. J. (2005). Calibration of Silicon Atomic Force Microscope Cantilevers. Nanotechnology 16 (2), 234-238. doi:10.1088/ 0957-4484/16/2/009

Glaser, D. E., Curtis, M. B., Sariano, P. A., Rollins, Z. A., Shergill, B. S., Anand, A., et al. (2022). Organ-on-a-chip Model of Vascularized Human Bone Marrow Niches. Biomaterials 280, 121245. doi:10.1016/j.biomaterials.2021.121245

Goldenberg, C., and Goldhirsch, I. (2005). Friction Enhances Elasticity in Granular Solids. Nature 435 (7039), 188-191. doi:10.1038/nature03497

Goldman, R. D., Pollack, R., and Hopkins, N. H. (1973). Preservation of Normal Behavior by Enucleated Cells in Culture. Proc. Natl. Acad. Sci. 70 (3), 750-754. doi:10.1073/pnas.70.3.750

Goossens, S., Vandamme, N., Van Vlierberghe, P., and Berx, G. (2017). EMT Transcription Factors in Cancer Development Re-evaluated: Beyond EMT and MET. Biochim. Biophys. Acta (Bba) - Rev. Cancer 1868 (2), 584-591. doi:10. 1016/j.bbcan.2017.06.006

Gordon, W. R., Zimmerman, B., He, L., Miles, L. J., Huang, J., Tiyanont, K., et al. (2015). Mechanical Allostery: Evidence for a Force Requirement in the Proteolytic Activation of Notch. Developmental Cel 33 (6), 729-736. doi:10. 1016/j.devcel.2015.05.004

Gossett, D. R., Tse, H. T. K., Lee, S. A., Ying, Y., Lindgren, A. G., Yang, O. O., et al. (2012). Hydrodynamic Stretching of Single Cells for Large Population Mechanical Phenotyping. Proc. Natl. Acad. Sci. 109 (20), 7630-7635. doi:10. 1073/pnas.1200107109

Goult, B. T. (2021). The Mechanical Basis of Memory - the MeshCODE Theory. Front. Mol. Neurosci. 14, 592951. doi:10.3389/fnmol.2021.592951

Goult, B. T., Zacharchenko, T., Bate, N., Tsang, R., Hey, F., Gingras, A. R., et al. (2013). RIAM and Vinculin Binding to Talin Are Mutually Exclusive and Regulate Adhesion Assembly and Turnover. J. Biol. Chem. 288 (12), 8238-8249. doi:10.1074/jbc.M112.438119

Graham, D. M., Andersen, T., Sharek, L., Uzer, G., Rothenberg, K., Hoffman, B. D., et al. (2018). Enucleated Cells Reveal Differential Roles of the Nucleus in Cell Migration, Polarity, and Mechanotransduction. J. Cel Biol. 217 (3), 895-914. doi:10.1083/jcb.201706097

Graham, D. M., and Burridge, K. (2016). Mechanotransduction and Nuclear Function. Curr. Opin. Cel Biol. 40, 98-105. doi:10.1016/j.ceb.2016.03.006

Gralka, M., and Kroy, K. (2015). Inelastic Mechanics: A Unifying Principle in Biomechanics. Biochim. Biophys. Acta (Bba) - Mol. Cel Res. 1853 (11), 3025-3037. doi:10.1016/j.bbamcr.2015.06.017

Grashoff, C., Hoffman, B. D., Brenner, M. D., Zhou, R., Parsons, M., Yang, M. T., et al. (2010). Measuring Mechanical Tension across Vinculin Reveals Regulation of Focal Adhesion Dynamics. Nature 466 (7303), 263-266. doi:10.1038/nature09198

Gruenbaum, Y., Margalit, A., Goldman, R. D., Shumaker, D. K., and Wilson, K. L. (2005). The Nuclear Lamina Comes of Age. Nat. Rev. Mol. Cel Biol. 6 (1), 21-31. doi:10.1038/nrm1550

Guck, J., Ananthakrishnan, R., Mahmood, H., Moon, T. J., Cunningham, C. C., and Käs, J. (2001). The Optical Stretcher: A Novel Laser Tool to Micromanipulate Cells. Biophysical J. 81 (2), 767-784. doi:10.1016/S0006-3495(01)75740-2

Guck, J., Schinkinger, S., Lincoln, B., Wottawah, F., Ebert, S., Romeyke, M., et al. (2005). Optical Deformability as an Inherent Cell Marker for Testing Malignant Transformation and Metastatic Competence. Biophysical J. 88 (5), 3689-3698. doi:10.1529/biophysj.104.045476

Gudipaty, S. A., Lindblom, J., Loftus, P. D., Redd, M. J., Edes, K., Davey, C. F., et al. (2017). Mechanical Stretch Triggers Rapid Epithelial Cell Division through Piezo1. Nature 543 (7643), 118-121. doi:10.1038/nature21407

Guelen, L., Pagie, L., Brasset, E., Meuleman, W., Faza, M. B., Talhout, W., et al. (2008). Domain Organization of Human Chromosomes Revealed by Mapping of Nuclear Lamina Interactions. Nature 453 (7197), 948-951. doi:10.1038/nature06947

Guevorkian, K., Colbert, M.-J., Durth, M., Dufour, S., and Brochard-Wyart, F. (2010). Aspiration of Biological Viscoelastic Drops. Phys. Rev. Lett. 104 (21), 218101. doi:10.1103/PhysRevLett.104.218101
Guglielmi, G., Barry, J. D., Huber, W., and De Renzis, S. (2015). An Optogenetic Method to Modulate Cell Contractility during Tissue Morphogenesis. Developmental Cel 35 (5), 646-660. doi:10.1016/j.devcel.2015.10.020

Guilak, F., Hayes, A. J., and Melrose, J. (2021). Perlecan in Pericellular Mechanosensory Cell-Matrix Communication, Extracellular Matrix Stabilisation and Mechanoregulation of Load-Bearing Connective Tissues. Int. J. Mol. Sci. 22 (5), 2716. doi:10.3390/ijms22052716

Guilluy, C., Osborne, L. D., Van Landeghem, L., Sharek, L., Superfine, R., GarciaMata, R., et al. (2014). Isolated Nuclei Adapt to Force and Reveal a Mechanotransduction Pathway in the Nucleus. Nat. Cel Biol. 16 (4), 376-381. doi:10.1038/ncb2927

Guilluy, C., Swaminathan, V., Garcia-Mata, R., Timothy O’Brien, E., Superfine, R., and Burridge, K. (2011). The Rho GEFs LARG and GEF-H1 Regulate the Mechanical Response to Force on Integrins. Nat. Cel Biol. 13 (6), 722-727. doi:10.1038/ncb2254

Guirao, B., and Bellaïche, Y. (2017). Biomechanics of Cell Rearrangements in Drosophila. Curr. Opin. Cel Biol. 48, 113-124. doi:10.1016/j.ceb.2017.06.004

Gundersen, G. G., and Worman, H. J. (2013). Nuclear Positioning. Cell 152 (6), 1376-1389. doi:10.1016/j.cell.2013.02.031

Guo, M., Ehrlicher, A. J., Jensen, M. H., Renz, M., Moore, J. R., Goldman, R. D., et al. (2014). Probing the Stochastic, Motor-Driven Properties of the Cytoplasm Using Force Spectrum Microscopy. Cell 158 (4), 822-832. doi:10.1016/j.cell. 2014.06.051

Haase, K., and Pelling, A. E. (2015). Investigating Cell Mechanics with Atomic Force Microscopy. J. R. Soc. Interf. 12 (104), 20140970. doi:10.1098/rsif.2014. 0970

Hackett, T.-L., and Knight, D. A. (2007). The Role of Epithelial Injury and Repair in the Origins of Asthma. Curr. Opin. Allergy Clin. Immunol. 7 (1), 63-68. doi:10. 1097/ACI.0b013e328013d61b

Haga, H., Sasaki, S., Kawabata, K., Ito, E., Ushiki, T., and Sambongi, T. (2000). Elasticity Mapping of Living Fibroblasts by AFM and Immunofluorescence Observation of the Cytoskeleton. Ultramicroscopy 82 (1-4), 253-258. doi:10. 1016/S0304-3991(99)00157-6

Haining, A. W. M., Rahikainen, R., Cortes, E., Lachowski, D., Rice, A., von Essen, M., et al. (2018). Mechanotransduction in Talin through the Interaction of the R8 Domain with DLC1. PLOS Biol. 16 (7), e2005599. doi:10.1371/journal.pbio. 2005599

Haisler, W. L., Timm, D. M., Gage, J. A., Tseng, H., Killian, T. C., and Souza, G. R. (2013). Three-dimensional Cell Culturing by Magnetic Levitation. Nat. Protoc. 8 (10), 1940-1949. doi:10.1038/nprot.2013.125

Hale, C. M., Shrestha, A. L., Khatau, S. B., Stewart-Hutchinson, P. J., Hernandez, L., Stewart, C. L., et al. (2008). Dysfunctional Connections between the Nucleus and the Actin and Microtubule Networks in Laminopathic Models. Biophysical J. 95 (11), 5462-5475. doi:10.1529/biophysj.108.139428

Hall, A. (2009). The Cytoskeleton and Cancer. Cancer Metastasis Rev. 28 (1-2), 5-14. doi:10.1007/s10555-008-9166-3

Han, M. K. L., and de Rooij, J. (2016). Converging and Unique Mechanisms of Mechanotransduction at Adhesion Sites. Trends Cel Biol. 26 (8), 612-623. doi:10.1016/j.tcb.2016.03.005

Han, S. J., Azarova, E. V., Whitewood, A. J., Bachir, A., Guttierrez, E., Groisman, A., et al. (2021). Pre-complexation of Talin and Vinculin without Tension Is Required for Efficient Nascent Adhesion Maturation. ELife 10, e66151. doi:10. 7554/eLife.66151

Hantos, Z., Daroczy, B., Suki, B., Nagy, S., and Fredberg, J. J. (1992). Input Impedance and Peripheral Inhomogeneity of Dog Lungs. J. Appl. Physiol. 72 (1), 168-178. doi:10.1152/jappl.1992.72.1.168

Harris, A. R., Daeden, A., and Charras, G. T. (2014). Formation of Adherens Junctions Leads to the Emergence of a Tissue-Level Tension in Epithelial Monolayers. J. Cel Sci. jcs, 142349. doi:10.1242/jcs.142349

Harris, A. R., Peter, L., Bellis, J., Baum, B., Kabla, A. J., and Charras, G. T. (2012a). Characterizing the Mechanics of Cultured Cell Monolayers. Proc. Natl. Acad. Sci. 109 (41), 16449-16454. doi:10.1073/pnas.1213301109

Harris, T. H., Banigan, E. J., Christian, D. A., Konradt, C., Tait Wojno, E. D., Norose, K., et al. (2012b). Generalized Lévy Walks and the Role of Chemokines in Migration of Effector CD8+ T Cells. Nature 486 (7404), 545-548. doi:10. 1038/nature11098

Hawkins, T., Mirigian, M., Selcuk Yasar, M., and Ross, J. L. (2010). Mechanics of Microtubules. J. Biomech. 43 (1), 23-30. doi:10.1016/j.jbiomech.2009.09.005 
Hay, E. D. (2005). The Mesenchymal Cell, its Role in the Embryo, and the Remarkable Signaling Mechanisms that Create it. Developmental Dyn. 233 (3), 706-720. doi:10.1002/dvdy.20345

Hayer, A., Shao, L., Chung, M., Joubert, L.-M., Yang, H. W., Tsai, F.-C., et al. (2016). Engulfed Cadherin Fingers Are Polarized Junctional Structures between Collectively Migrating Endothelial Cells. Nat. Cel Biol. 18 (12), 1311-1323. doi: $10.1038 / \mathrm{ncb} 3438$

Hazan, R. B., Qiao, R., Keren, R., Badano, I., and Suyama, K. (2004). Cadherin Switch in Tumor Progression. Ann. New York Acad. Sci. 1014 (1), 155-163. doi:10.1196/annals.1294.016

He, L., Si, G., Huang, J., Samuel, A. D. T., and Perrimon, N. (2018). Mechanical Regulation of Stem-Cell Differentiation by the Stretch-Activated Piezo Channel. Nature 555 (7694), 103-106. doi:10.1038/nature25744

Heck, J. N., Ponik, S. M., Garcia-Mendoza, M. G., Pehlke, C. A., Inman, D. R., Eliceiri, K. W., et al. (2012). Microtubules Regulate GEF-H1 in Response to Extracellular Matrix Stiffness. Mol. Biol. Cel 23 (13), 2583-2592. doi:10.1091/ mbc.e11-10-0876

Heguy, A., Harvey, B.-G., Leopold, P. L., Dolgalev, I., Raman, T., and Crystal, R. G. (2007). Responses of the Human Airway Epithelium Transcriptome to In Vivo Injury. Physiol. Genomics 29 (2), 139-148. doi:10.1152/physiolgenomics.00167. 2006

Hejna, M., Jorapur, A., Song, J. S., and Judson, R. L. (2017). High Accuracy Labelfree Classification of Single-Cell Kinetic States from Holographic Cytometry of Human Melanoma Cells. Scientific Rep. 7 (1), 11943. doi:10.1038/s41598-01712165-1

Helmke, B. P., Rosen, A. B., and Davies, P. F. (2003). Mapping Mechanical Strain of an Endogenous Cytoskeletal Network in Living Endothelial Cells. Biophysical J. 84 (4), 2691-2699. doi:10.1016/S0006-3495(03)75074-7

Helmlinger, G., Netti, P. A., Lichtenbeld, H. C., Melder, R. J., and Jain, R. K. (1997). Solid Stress Inhibits the Growth of Multicellular Tumor Spheroids. Nat. Biotechnol. 15 (8), 778-783. doi:10.1038/nbt0897-778

Henak, C. R., Ross, K. A., Bonnevie, E. D., Fortier, L. A., Cohen, I., Kennedy, J. G., et al. (2016). Human Talar and Femoral Cartilage Have Distinct Mechanical Properties Near the Articular Surface. J. Biomech. 49 (14), 3320-3327. doi:10. 1016/j.jbiomech.2016.08.016

Henkes, S., Fily, Y., and Marchetti, M. C. (2011). Active Jamming: Self-Propelled Soft Particles at High Density. Phys. Rev. E 84 (4), 040301. doi:10.1103/ PhysRevE.84.040301

Hertz, H. (1882). Ueber die Berührung fester elastischer Körper. J. Für Die Reine Angew. Mathematik (Crelles Journal) 1882 (92), 156-171. doi:10.1515/crll.1882. 92.156

Ho, C. Y., and Lammerding, J. (2012). Lamins at a Glance. J. Cel Sci. 125 (9), 2087-2093. doi: $10.1242 /$ jcs. 087288

Hochmuth, R. M. (2000). Micropipette Aspiration of Living Cells. J. Biomech. 33 (1), 15-22. doi:10.1016/S0021-9290(99)00175-X

Holgate, S. T. (2004). Epithelial-Mesenchymal Communication in the Pathogenesis of Chronic Asthma. Proc. Am. Thorac. Soc. 1 (2), 93-98. doi:10.1513/pats.2306034

Horton, E. R., Astudillo, P., Humphries, M. J., and Humphries, J. D. (2016). Mechanosensitivity of Integrin Adhesion Complexes: Role of the Consensus Adhesome. Exp. Cel Res. 343 (1), 7-13. doi:10.1016/j.yexcr.2015.10.025

Horton, E. R., Byron, A., Askari, J. A., Ng, D. H. J., Millon-Frémillon, A., Robertson, J., et al. (2015). Definition of a Consensus Integrin Adhesome and its Dynamics during Adhesion Complex Assembly and Disassembly. Nat. Cel Biol. 17 (12), 1577-1587. doi:10.1038/ncb3257

Houk, A. R., Jilkine, A., Mejean, C. O., Boltyanskiy, R., Dufresne, E. R., Angenent, S. B., et al. (2012). Membrane Tension Maintains Cell Polarity by Confining Signals to the Leading Edge during Neutrophil Migration. Cell 148 (1-2), 175-188. doi:10.1016/j.cell.2011.10.050

Hu, J., Jafari, S., Han, Y., Grodzinsky, A. J., Cai, S., and Guo, M. (2017). Size- and Speed-dependent Mechanical Behavior in Living Mammalian Cytoplasm. Proc. Natl. Acad. Sci. 114 (36), 9529-9534. doi:10.1073/pnas.1702488114

Huang, D. L., Bax, N. A., Buckley, C. D., Weis, W. I., and Dunn, A. R. (2017). Vinculin Forms a Directionally Asymmetric Catch Bond with F-Actin. Science 357 (6352), 703-706. doi:10.1126/science.aan2556

Hubaud, A., Regev, I., Mahadevan, L., and Pourquié, O. (2017). Excitable Dynamics and Yap-dependent Mechanical Cues Drive the Segmentation Clock. Cell 171 (3), 668-682. e11. doi:10.1016/j.cell.2017.08.043
Huber, F., Schnauß, J., Rönicke, S., Rauch, P., Müller, K., Fütterer, C., et al. (2013). Emergent Complexity of the Cytoskeleton: From Single Filaments to Tissue. Adv. Phys. 62 (1), 1-112. doi:10.1080/00018732.2013.771509

Humphrey, D., Duggan, C., Saha, D., Smith, D., and Käs, J. (2002). Active Fluidization of Polymer Networks through Molecular Motors. Nature 416 (6879), 413-416. doi:10.1038/416413a

Hung, W.-C., Yang, J. R., Yankaskas, C. L., Wong, B. S., Wu, P.-H., Pardo-Pastor, C., et al. (2016). Confinement Sensing and Signal Optimization via Piezo1/PKA and Myosin II Pathways. Cel Rep. 15 (7), 1430-1441. doi:10.1016/j.celrep.2016. 04.035

Hunter, G. L., He, L., Perrimon, N., Charras, G., Giniger, E., and Baum, B. (2019). A Role for Actomyosin Contractility in Notch Signaling. BMC Biol. 17 (1), 12. doi:10.1186/s12915-019-0625-9

Hunter, G. L., and Weeks, E. R. (2012). The Physics of the Colloidal Glass Transition. Rep. Prog. Phys. 75 (6), 066501. doi:10.1088/0034-4885/75/6/066501

Hutter, J. L., and Bechhoefer, J. (1993). Calibration of Atomic-force Microscope Tips. Rev. Scientific Instr. 64 (7), 1868-1873. doi:10.1063/1.1143970

Huwart, L., Peeters, F., Sinkus, R., Annet, L., Salameh, N., ter Beek, L. C., et al. (2006). Liver Fibrosis: Non-invasive Assessment with MR Elastography. NMR Biomed. 19 (2), 173-179. doi:10.1002/nbm.1030

Huynh, J., Nishimura, N., Rana, K., Peloquin, J. M., Califano, J. P., Montague, C. R., et al. (2011). Age-Related Intimal Stiffening Enhances Endothelial Permeability and Leukocyte Transmigration. Sci. Translational Med. 3 (112). doi:10.1126/ scitranslmed.3002761

Idevall-Hagren, O., Dickson, E. J., Hille, B., Toomre, D. K., and De Camilli, P. (2012). Optogenetic Control of Phosphoinositide Metabolism. Proc. Natl. Acad. Sci. 109 (35), E2316-E2323. doi:10.1073/pnas.1211305109

Ilina, O., Gritsenko, P. G., Syga, S., Lippoldt, J., La Porta, C. A. M., Chepizhko, O., et al. (2020). Cell-cell Adhesion and 3D Matrix Confinement Determine Jamming Transitions in Breast Cancer Invasion. Nat. Cel Biol. 22 (9), 1103-1115. doi:10.1038/s41556-020-0552-6

Inaki, M., Vishnu, S., Cliffe, A., and Rorth, P. (2012). Effective Guidance of Collective Migration Based on Differences in Cell States. Proc. Natl. Acad. Sci. 109 (6), 2027-2032. doi:10.1073/pnas.1115260109

Inch, W. R., McCredie, J. A., and Sutherland, R. M. (1970). Growth of Nodular Carcinomas in Rodents Compared with Multi-Cell Spheroids in Tissue Culture. Growth 34 (3), 271-282.

Ingber, D. E. (1997). Tensegrity: The Architectural Basis of Cellular Mechanotransduction. Аnnu. Rev. Physiol. 59 (1), 575-599. doi:10.1146/ annurev.physiol.59.1.575

Iordan, A., Duperray, A., Gérard, A., Grichine, A., and Verdier, C. (2010). Breakdown of Cell-Collagen Networks through Collagen Remodeling. Biorheology 47 (5-6), 277-295. doi:10.3233/BIR-2010-0575

Irvine, D. J., Hue, K.-A., Mayes, A. M., and Griffith, L. G. (2002). Simulations of Cell-Surface Integrin Binding to Nanoscale-Clustered Adhesion Ligands. Biophysical J. 82 (1), 120-132. doi:10.1016/S0006-3495(02)75379-4

Irvine, D. J., Ruzette, A.-V. G., Mayes, A. M., and Griffith, L. G. (2001). Nanoscale Clustering of RGD Peptides at Surfaces Using Comb Polymers. 2. Surface Segregation of Comb Polymers in Polylactide. Biomacromolecules 2 (2), 545-556. doi:10.1021/bm015510f

Jaalouk, D. E., and Lammerding, J. (2009). Mechanotransduction Gone Awry. Nat. Rev. Mol. Cel Biol. 10 (1), 63-73. doi:10.1038/nrm2597

Jain, N., Iyer, K. V., Kumar, A., and Shivashankar, G. V. (2013). Cell Geometric Constraints Induce Modular Gene-Expression Patterns via Redistribution of HDAC3 Regulated by Actomyosin Contractility. Proc. Natl. Acad. Sci. 110 (28), 11349-11354. doi:10.1073/pnas.1300801110

Jansen, K. A., Atherton, P., and Ballestrem, C. (2017). Mechanotransduction at the Cell-Matrix Interface. Semin. Cel Developmental Biol. 71, 75-83. doi:10.1016/j. semcdb.2017.07.027

Jeon, J. S., Bersini, S., Gilardi, M., Dubini, G., Charest, J. L., Moretti, M., et al. (2015). Human 3D Vascularized Organotypic Microfluidic Assays to Study Breast Cancer Cell Extravasation. Proc. Natl. Acad. Sci. 112 (1), 214-219. doi:10. 1073/pnas.1417115112

Jeon, N. L., Dertinger, S. K. W., Chiu, D. T., Choi, I. S., Stroock, A. D., and Whitesides, G. M. (2000). Generation of Solution and Surface Gradients Using Microfluidic Systems. Langmuir 16 (22), 8311-8316. doi:10.1021/la000600b

Jo, M., Lester, R. D., Montel, V., Eastman, B., Takimoto, S., and Gonias, S. L. (2009). Reversibility of Epithelial-Mesenchymal Transition (EMT) Induced in Breast 
Cancer Cells by Activation of Urokinase Receptor-dependent Cell Signaling. J. Biol. Chem. 284 (34), 22825-22833. doi:10.1074/jbc.M109.023960

Jordan, P., Kerdok, A. E., Howe, R. D., and Socrate, S. (2011). Identifying a Minimal Rheological Configuration: A Tool for Effective and Efficient Constitutive Modeling of Soft Tissues. J. Biomechanical Eng. 133 (4), 041006. doi:10. $1115 / 1.4003620$

Ju, L., Dong, J., Cruz, M. A., and Zhu, C. (2013). The N-terminal Flanking Region of the A1 Domain Regulates the Force-dependent Binding of von Willebrand Factor to Platelet Glycoprotein Iba. J. Biol. Chem. 288 (45), 32289-32301. doi:10.1074/jbc.M113.504001

June, R. K., Ly, S., and Fyhrie, D. P. (2009). Cartilage Stress-Relaxation Proceeds Slower at Higher Compressive Strains. Arch. Biochem. Biophys. 483 (1), 75-80. doi:10.1016/j.abb.2008.11.029

Kalwarczyk, T., Zibacz, N., Bielejewska, A., Zaboklicka, E., Koynov, K., Szymański, J., et al. (2011). Comparative Analysis of Viscosity of Complex Liquids and Cytoplasm of Mammalian Cells at the Nanoscale. Nano Lett. 11 (5), 2157-2163. doi: $10.1021 / \mathrm{nl} 2008218$

Kandel, M. E., Fernandes, D., Taylor, A. M., Shakir, H., Best-Popescu, C., and Popescu, G. (2017). Three-dimensional Intracellular Transport in Neuron Bodies and Neurites Investigated by Label-free Dispersion-relation Phase Spectroscopy. Cytometry A 91 (5), 519-526. doi:10.1002/cyto.a.23081

Kang, H., Wen, Q., Janmey, P. A., Tang, J. X., Conti, E., and MacKintosh, F. C. (2009). Nonlinear Elasticity of Stiff Filament Networks: Strain Stiffening, Negative Normal Stress, and Filament Alignment in Fibrin Gels. The J. Phys. Chem. B 113 (12), 3799-3805. doi:10.1021/jp807749f

Kapałczyńska, M., Kolenda, T., Przybyła, W., Zajączkowska, M., Teresiak, A., Filas, V., et al. (2016). 2D and 3D Cell Cultures - a Comparison of Different Types of Cancer Cell Cultures. Arch. Med. Sci. doi:10.5114/aoms.2016.63743

Karcher, H., Lammerding, J., Huang, H., Lee, R. T., Kamm, R. D., and KaazempurMofrad, M. R. (2003). A Three-Dimensional Viscoelastic Model for Cell Deformation with Experimental Verification. Biophysical J. 85 (5), 3336-3349. doi:10.1016/S0006-3495(03)74753-5

Kaverina, I., Krylyshkina, O., Beningo, K., Anderson, K., Wang, Y.-L., and Small, J. V. (2002). Tensile Stress Stimulates Microtubule Outgrowth in Living Cells. J. Cel Sci. 115 (Pt 11), 2283-2291.

Kawai, K., Yamaga, M., Iwamae, Y., Kiyota, M., Kamata, H., Hirata, H., et al. (2004). A PLC 81 -Binding Protein, p122RhoGAP, Is Localized in Focal Adhesions. Biochem. Soc. Trans. 32 (6), 1107-1109. doi:10.1042/BST0321107

Kefauver, J. M., Ward, A. B., and Patapoutian, A. (2020). Discoveries in Structure and Physiology of Mechanically Activated Ion Channels. Nature 587 (7835), 567-576. doi:10.1038/s41586-020-2933-1

Kelley, C. F., Litschel, T., Schumacher, S., Dedden, D., Schwille, P., and Mizuno, N. (2020). Phosphoinositides Regulate Force-independent Interactions between Talin, Vinculin, and Actin. ELife 9, e56110. doi:10.7554/eLife.56110

Ketene, A. N., Schmelz, E. M., Roberts, P. C., and Agah, M. (2012). The Effects of Cancer Progression on the Viscoelasticity of Ovarian Cell Cytoskeleton Structures. Nanomedicine: Nanotechnology, Biol. Med. 8 (1), 93-102. doi:10. 1016/j.nano.2011.05.012

Khalili, A., and Ahmad, M. (2015). A Review of Cell Adhesion Studies for Biomedical and Biological Applications. Int. J. Mol. Sci. 16 (8), 18149-18184. doi:10.3390/ijms160818149

Khan, R. B., and Goult, B. T. (2019). Adhesions Assemble!-Autoinhibition as a Major Regulatory Mechanism of Integrin-Mediated Adhesion. Front. Mol. Biosciences 6, 144. doi:10.3389/fmolb.2019.00144

Khatibzadeh, N., Spector, A. A., Brownell, W. E., and Anvari, B. (2013). Effects of Plasma Membrane Cholesterol Level and Cytoskeleton F-Actin on Cell Protrusion Mechanics. PLoS ONE 8 (2), e57147. doi:10.1371/journal.pone. 0057147

Kießling, T. R., Stange, R., Käs, J. A., and Fritsch, A. W. (2013). Thermorheology of Living Cells-Impact of Temperature Variations on Cell Mechanics. New J. Phys. 15 (4), 045026. doi:10.1088/1367-2630/15/4/045026

Kim, H. Y., Jackson, T. R., and Davidson, L. A. (2017). On the Role of Mechanics in Driving Mesenchymal-To-Epithelial Transitions. Semin. Cel Developmental Biol. 67, 113-122. doi:10.1016/j.semcdb.2016.05.011

Kim, J.-Y., Fluri, D. A., Marchan, R., Boonen, K., Mohanty, S., Singh, P., et al. (2015). 3D Spherical Microtissues and Microfluidic Technology for MultiTissue Experiments and Analysis. J. Biotechnol. 205, 24-35. doi:10.1016/j. jbiotec.2015.01.003
Kim, S., Kim, H. J., and Jeon, N. L. (2010). Biological Applications of Microfluidic Gradient Devices. Integr. Biol. 2 (11-12), 584. doi:10.1039/c0ib00055h

Kimura, K., Ito, M., Amano, M., Chihara, K., Fukata, Y., Nakafuku, M., et al. (1996). Regulation of Myosin Phosphatase by Rho and Rho-Associated Kinase (Rho-Kinase). Science 273 (5272), 245-248. doi:10.1126/science.273.5272.245

Kirby, T. J., and Lammerding, J. (2018). Emerging Views of the Nucleus as a Cellular Mechanosensor. Nat. Cel Biol. 20 (4), 373-381. doi:10.1038/s41556018-0038-y

Kirschner, M. (1986). Beyond Self-Assembly: From Microtubules to Morphogenesis. Cell 45 (3), 329-342. doi:10.1016/0092-8674(86)90318-1

Kishikawa, M., Suzuki, A., and Ohno, S. (2008). APKC Enables Development of Zonula Adherens by Antagonizing Centripetal Contraction of the Circumferential Actomyosin Cables. J. Cel Sci. 121 (15), 2481-2492. doi:10. $1242 /$ jcs. 024109

Ko, J., Ahn, J., Kim, S., Lee, Y., Lee, J., Park, D., et al. (2019). Tumor Spheroid-OnA-Chip: A Standardized Microfluidic Culture Platform for Investigating Tumor Angiogenesis. Lab. A Chip 19 (17), 2822-2833. doi:10.1039/C9LC00140A

Kole, T. P., Tseng, Y., Jiang, I., Katz, J. L., and Wirtz, D. (2005). Intracellular Mechanics of Migrating Fibroblasts. Mol. Biol. Cel 16 (1), 328-338. doi:10.1091/ mbc.e04-06-0485

Kollmannsberger, P., and Fabry, B. (2007). BaHigh-force Magnetic Tweezers with Force Feedback for Biological Applications. Rev. Scientific Instr. 78 (11), 114301. doi:10.1063/1.2804771

Kollmannsberger, P., Mierke, C. T., and Fabry, B. (2011). Nonlinear Viscoelasticity of Adherent Cells Is Controlled by Cytoskeletal Tension. Soft Matter 7 (7), 3127-3132. doi:10.1039/COSM00833H

Kong, F., García, A. J., Mould, A. P., Humphries, M. J., and Zhu, C. (2009). Demonstration of Catch Bonds between an Integrin and its Ligand. J. Cel Biol. 185 (7), 1275-1284. doi:10.1083/jcb.200810002

Kourtidis, A., Lu, R., Pence, L. J., and Anastasiadis, P. Z. (2017). A central Role for Cadherin Signaling in Cancer. Exp. Cel Res. 358 (1), 78-85. doi:10.1016/j.yexcr. 2017.04.006

Krause, M., and Gautreau, A. (2014). Steering Cell Migration: Lamellipodium Dynamics and the Regulation of Directional Persistence. Nat. Rev. Mol. Cel Biol. 15 (9), 577-590. doi:10.1038/nrm3861

Krause, M., te Riet, J., and Wolf, K. (2013). Probing the Compressibility of Tumor Cell Nuclei by Combined Atomic Force-Confocal Microscopy. Phys. Biol. 10 (6), 065002. doi:10.1088/1478-3975/10/6/065002

Krause, M., and Wolf, K. (2015). Cancer Cell Migration in 3D Tissue: Negotiating Space by Proteolysis and Nuclear Deformability. Cel Adhes. Migration 9 (5), 357-366. doi:10.1080/19336918.2015.1061173

Krendel, M., Gloushankova, N. A., Bonder, E. M., Feder, H. H., Vasiliev, J. M., and Gelfand, I. M. (1999). Myosin-dependent Contractile Activity of the Actin Cytoskeleton Modulates the Spatial Organization of Cell-Cell Contacts in Cultured Epitheliocytes. Proc. Natl. Acad. Sci. 96 (17), 9666-9670. doi:10. 1073/pnas.96.17.9666

Kucharová, M., Doubal, S., Klemera, P., Rejchrt, P., and Navrátil, M. (2007). Viscoelasticity of Biological Materials-Measurement and Practical Impact on Biomedicine. Physiol. Res. 56 (Suppl. 1), S33-S37.

Kuimova, M. K., Yahioglu, G., Levitt, J. A., and Suhling, K. (2008). Molecular Rotor Measures Viscosity of Live Cells via Fluorescence Lifetime Imaging. J. Am. Chem. Soc. 130 (21), 6672-6673. doi:10.1021/ja800570d

Kukulski, F., Ben Yebdri, F., Lefebvre, J., Warny, M., Tessier, P. A., and Sévigny, J. (2007). Extracellular Nucleotides Mediate LPS-Induced Neutrophil Migration In Vitro and In Vivo. J. Leukoc. Biol. 81 (5), 1269-1275. doi:10.1189/jlb.1206758

Kumar, A., Ouyang, M., Van den Dries, K., McGhee, E. J., Tanaka, K., Anderson, M. D., et al. (2016). Talin Tension Sensor Reveals Novel Features of Focal Adhesion Force Transmission and Mechanosensitivity. J. Cel Biol. 213 (3), 371-383. doi:10.1083/jcb.201510012

Kumar, S., and Weaver, V. M. (2009). Mechanics, Malignancy, and Metastasis: The Force Journey of a Tumor Cell. Cancer Metastasis Rev. 28 (1-2), 113-127. doi:10.1007/s10555-008-9173-4

Kunschmann, T., Puder, S., Fischer, T., Perez, J., Wilharm, N., and Mierke, C. T. (2017). Integrin-linked Kinase Regulates Cellular Mechanics Facilitating the Motility in 3D Extracellular Matrices. Biochim. Biophys. Acta (Bba) - Mol. Cel Res. 1864 (3), 580-593. doi:10.1016/j.bbamcr.2016.12.019

Kunschmann, T., Puder, S., Fischer, T., Steffen, A., Rottner, K., and Mierke, C. T. (2019). The Small GTPase Rac1 Increases Cell Surface Stiffness and Enhances 
3D Migration into Extracellular Matrices. Scientific Rep. 9 (1), 7675. doi:10. 1038/s41598-019-43975-0

Kuriyama, S., Theveneau, E., Benedetto, A., Parsons, M., Tanaka, M., Charras, G., et al. (2014). In Vivo collective Cell Migration Requires an LPAR2-dependent Increase in Tissue Fluidity. J. Cel Biol. 206 (1), 113-127. doi:10.1083/jcb. 201402093

Kwon, S., Yang, W., Moon, D., and Kim, K. S. (2020). Biomarkers to Quantify Cell Migration Characteristics. Cancer Cel Int. 20 (1), 217. doi:10.1186/s12935-02001312-w

Lai, Y., and Hu, Y. (2017). Unified Solution for Poroelastic Oscillation Indentation on Gels for Spherical, Conical and Cylindrical Indenters. Soft Matter 13 (4), 852-861. doi:10.1039/C6SM02341J

Lammerding, J., Fong, L. G., Ji, J. Y., Reue, K., Stewart, C. L., Young, S. G., et al. (2006). Lamins A and C but Not Lamin B1 Regulate Nuclear Mechanics. J. Biol. Chem. 281 (35), 25768-25780. doi:10.1074/jbc.M513511200

Lammerding, J., Hsiao, J., Schulze, P. C., Kozlov, S., Stewart, C. L., and Lee, R. T. (2005). Abnormal Nuclear Shape and Impaired Mechanotransduction in Emerin-Deficient Cells. J. Cel Biol. 170 (5), 781-791. doi:10.1083/jcb.200502148

Lammerding, J., Schulze, P. C., Takahashi, T., Kozlov, S., Sullivan, T., Kamm, R. D., et al. (2004). Lamin A/C Deficiency Causes Defective Nuclear Mechanics and Mechanotransduction. J. Clin. Invest. 113 (3), 370-378. doi:10.1172/ JCI200419670

Lampo, T. J., Kennard, A. S., and Spakowitz, A. J. (2016). Physical Modeling of Dynamic Coupling between Chromosomal Loci. Biophysical J. 110 (2), 338-347. doi:10.1016/j.bpj.2015.11.3520

Lancaster, M. A., and Knoblich, J. A. (2014). Organogenesis in a Dish: Modeling Development and Disease Using Organoid Technologies. Science 345 (6194), 1247125. doi:10.1126/science.1247125

Lange, J. R., and Fabry, B. (2013). Cell and Tissue Mechanics in Cell Migration. Exp. Cel Res. 319 (16), 2418-2423. doi:10.1016/j.yexcr.2013.04.023

Langridge, P. D., and Struhl, G. (2017). Epsin-Dependent Ligand Endocytosis Activates Notch by Force. Cell 171 (6), 1383-1396. doi:10.1016/j.cell.2017. 10.048

Lauffenburger, D. A., and Horwitz, A. F. (1996). Cell Migration: A Physically Integrated Molecular Process. Cell 84 (3), 359-369. doi:10.1016/S00928674(00)81280-5

Laurent, V. M., Kasas, S., Yersin, A., Schäffer, T. E., Catsicas, S., Dietler, G., et al. (2005). Gradient of Rigidity in the Lamellipodia of Migrating Cells Revealed by Atomic Force Microscopy. Biophysical J. 89 (1), 667-675. doi:10.1529/biophysj. 104.052316

Lawless, B. M., Sadeghi, H., Temple, D. K., Dhaliwal, H., Espino, D. M., and Hukins, D. W. L. (2017). Viscoelasticity of Articular Cartilage: Analysing the Effect of Induced Stress and the Restraint of Bone in a Dynamic Environment. J. Mech. Behav. Biomed. Mater. 75, 293-301. doi:10.1016/j.jmbbm.2017.07.040

Lawson, N. D., Vogel, A. M., and Weinstein, B. M. (2002). Sonic Hedgehog and Vascular Endothelial Growth Factor Act Upstream of the Notch Pathway during Arterial Endothelial Differentiation. Developmental Cel 3 (1), 127-136. doi:10.1016/S1534-5807(02)00198-3

Leckband, D. E., and de Rooij, J. (2014). Cadherin Adhesion and Mechanotransduction. Annu. Rev. Cel Developmental Biol. 30 (1), 291-315. doi:10.1146/annurev-cellbio-100913-013212

Lecuit, T., Lenne, P.-F., and Munro, E. (2011). Force Generation, Transmission, and Integration during Cell and Tissue Morphogenesis. Annu. Rev. Cel Developmental Biol. 27 (1), 157-184. doi:10.1146/annurev-cellbio-100109-104027

Lee, H.-S., Anekal, P., Lim, C. J., Liu, C.-C., and Ginsberg, M. H. (2013). Two Modes of Integrin Activation Form a Binary Molecular Switch in Adhesion Maturation. Mol. Biol. Cel 24 (9), 1354-1362. doi:10.1091/mbc.e12-09-0695

Lee, J. S. H., Hale, C. M., Panorchan, P., Khatau, S. B., George, J. P., Tseng, Y., et al. (2007). Nuclear Lamin A/C Deficiency Induces Defects in Cell Mechanics, Polarization, and Migration. Biophysical J. 93 (7), 2542-2552. doi:10.1529/ biophysj.106.102426

Lee, K., Gjorevski, N., Boghaert, E., Radisky, D. C., and Nelson, C. M. (2011). Snail1, Snail2, and E47 Promote Mammary Epithelial Branching Morphogenesis: Transcription Factors in Branching Morphogenesis. EMBO J. 30 (13), 2662-2674. doi:10.1038/emboj.2011.159

Lee, K., and Nelson, C. M. (2012). New Insights into the Regulation of Epithelial-Mesenchymal Transition and Tissue Fibrosis. Int. Rev. Cel Mol. Biol. 294, 171-221. doi:10.1016/B978-0-12-394305-7.00004-5
Leggett, S. E., Hruska, A. M., Guo, M., and Wong, I. Y. (2021). The EpithelialMesenchymal Transition and the Cytoskeleton in Bioengineered Systems. Cell Commun. Signaling 19 (1), 32. doi:10.1186/s12964-021-00713-2

Lehmann, W., Mossmann, D., Kleemann, J., Mock, K., Meisinger, C., Brummer, T., et al. (2016). ZEB1 Turns into a Transcriptional Activator by Interacting with YAP1 in Aggressive Cancer Types. Nat. Commun. 7 (1), 10498. doi:10.1038/ ncomms 10498

Lekka, M., Pogoda, K., Gostek, J., Klymenko, O., Prauzner-Bechcicki, S., Wiltowska-Zuber, J., et al. (2012). Cancer Cell Recognition - Mechanical Phenotype. Micron 43 (12), 1259-1266. doi:10.1016/j.micron.2012.01.019

Lessey-Morillon, E. C., Osborne, L. D., Monaghan-Benson, E., Guilluy, C., O’Brien, E. T., Superfine, R., et al. (2014). The RhoA Guanine Nucleotide Exchange Factor, LARG, Mediates ICAM-1-dependent Mechanotransduction in Endothelial Cells to Stimulate Transendothelial Migration. J. Immunol. 192 (7), 3390-3398. doi:10.4049/jimmunol.1302525

Levskaya, A., Weiner, O. D., Lim, W. A., and Voigt, C. A. (2009). Spatiotemporal Control of Cell Signalling Using a Light-Switchable Protein Interaction. Nature 461 (7266), 997-1001. doi:10.1038/nature08446

Li, B., and Sun, S. X. (2014). Coherent Motions in Confluent Cell Monolayer Sheets. Biophysical J. 107 (7), 1532-1541. doi:10.1016/j.bpj.2014.08.006

Li, J., and Springer, T. A. (2018). Energy Landscape Differences Among Integrins Establish the Framework for Understanding Activation. J. Cel Biol. 217 (1), 397-412. doi:10.1083/jcb.201701169

Li, Q. S., Lee, G. Y. H., Ong, C. N., and Lim, C. T. (2008). AFM Indentation Study of Breast Cancer Cells. Biochem. Biophysical Res. Commun. 374 (4), 609-613. doi:10.1016/j.bbrc.2008.07.078

Li, R., Liang, J., Ni, S., Zhou, T., Qing, X., Li, H., et al. (2010). A Mesenchymal-ToEpithelial Transition Initiates and Is Required for the Nuclear Reprogramming of Mouse Fibroblasts. Cell Stem Cell 7 (1), 51-63. doi:10.1016/j.stem.2010.04.01

Li, S., Qin, X., Chai, S., Qu, C., Wang, X., and Zhang, H. (2016). Modulation of E-Cadherin Expression Promotes Migration Ability of Esophageal Cancer Cells. Scientific Rep. 6 (1), 21713. doi:10.1038/srep21713

Li, Y., Schnekenburger, J., and Duits, M. H. G. (2009). Intracellular Particle Tracking as a Tool for Tumor Cell Characterization. J. Biomed. Opt. 14 (6), 064005. doi:10.1117/1.3257253

Lieleg, O., Schmoller, K. M., Claessens, M. M. A. E., and Bausch, A. R. (2009). Cytoskeletal Polymer Networks: Viscoelastic Properties Are Determined by the Microscopic Interaction Potential of Cross-Links. Biophysical J. 96 (11), 4725-4732. doi:10.1016/j.bpj.2009.03.038

Ligon, S. C., Liska, R., Stampfl, J., Gurr, M., and Mülhaupt, R. (2017). Polymers for 3D Printing and Customized Additive Manufacturing. Chem. Rev. 117 (15), 10212-10290. doi:10.1021/acs.chemrev.7b00074

Lim, C. T., Zhou, E. H., and Quek, S. T. (2006). Mechanical Models for Living Cells-A Review. J. Biomech. 39 (2), 195-216. doi:10.1016/j.jbiomech.2004.12.008

Lin, S.-Z., Li, B., Xu, G.-K., and Feng, X.-Q. (2017). Collective Dynamics of Cancer Cells Confined in a Confluent Monolayer of normal Cells. J. Biomech. 52, 140-147. doi:10.1016/j.jbiomech.2016.12.035

Lincoln, B., Erickson, H. M., Schinkinger, S., Wottawah, F., Mitchell, D., Ulvick, S., et al. (2004). Deformability-based Flow Cytometry. Cytometry 59A (2), 203-209. doi:10.1002/cyto.a.20050

Lindberg, U., Karlsson, R., Lassing, I., Schutt, C. E., and Höglund, A.-S. (2008). The Microfilament System and Malignancy. Semin. Cancer Biol. 18 (1), 2-11. doi:10. 1016/j.semcancer.2007.10.002

Liu, C.-Y., Lin, H.-H., Tang, M.-J., and Wang, Y.-K. (2015a). Vimentin Contributes to Epithelial-Mesenchymal Transition Cancer Cell Mechanics by Mediating Cytoskeletal Organization and Focal Adhesion Maturation. Oncotarget 6 (18), 15966-15983. doi:10.18632/oncotarget.3862

Liu, C., He, J., Ruymbeke, E. van., Keunings, R., and Bailly, C. (2006). Evaluation of Different Methods for the Determination of the Plateau Modulus and the Entanglement Molecular Weight. Polymer 47 (13), 4461-4479. doi:10.1016/j. polymer.2006.04.054

Liu, F., Lagares, D., Choi, K. M., Stopfer, L., Marinković, A., Vrbanac, V., et al. (2015b). Mechanosignaling through YAP and TAZ Drives Fibroblast Activation and Fibrosis. Am. J. Physiology-Lung Cell Mol. Physiol. 308 (4), L344-L357. doi:10.1152/ajplung.00300.2014

Liu, W.-D., and Yang, B. (2017). Patterned Surfaces for Biological Applications: A New Platform Using Two Dimensional Structures as Biomaterials. Chin. Chem. Lett. 28 (4), 675-690. doi:10.1016/j.cclet.2016.09.004 
Liu, Z., and Bilston, L. E. (2002). Large Deformation Shear Properties of Liver Tissue. Biorheology 39 (6), 735-742.

Liu, Z., and Bilston, L. (2000). On the Viscoelastic Character of Liver Tissue: Experiments and Modelling of the Linear Behaviour. Biorheology 37 (3), 191-201.

Liu, Z., Tan, J. L., Cohen, D. M., Yang, M. T., Sniadecki, N. J., Ruiz, S. A., et al. (2010). Mechanical Tugging Force Regulates the Size of Cell-Cell Junctions. Proc. Natl. Acad. Sci. 107 (22), 9944-9949. doi:10.1073/pnas.0914547107

Loirand, G., and Pacaud, P. (2014). Involvement of Rho GTPases and Their Regulators in the Pathogenesis of Hypertension. Small GTPases 5 (4), e983866. doi:10.4161/sgtp.28846

Lombardi, M. L., Jaalouk, D. E., Shanahan, C. M., Burke, B., Roux, K. J., and Lammerding, J. (2011). The Interaction between Nesprins and Sun Proteins at the Nuclear Envelope Is Critical for Force Transmission between the Nucleus and Cytoskeleton. J. Biol. Chem. 286 (30), 26743-26753. doi:10.1074/jbc.M111. 233700

Lopez, B. J., and Valentine, M. T. (2015). Molecular Control of Stress Transmission in the Microtubule Cytoskeleton. Biochim. Biophys. Acta (Bba) - Mol. Cel Res. 1853 (11), 3015-3024. doi:10.1016/j.bbamcr.2015.07.016

López-Fagundo, C., Livi, L. L., Ramchal, T., Darling, E. M., and Hoffman-Kim, D. (2016). A Biomimetic Synthetic Feeder Layer Supports the Proliferation and Self-Renewal of Mouse Embryonic Stem Cells. Acta Biomater. 39, 55-64. doi:10. 1016/j.actbio.2016.04.047

Luca, V. C., Kim, B. C., Ge, C., Kakuda, S., Wu, D., Roein-Peikar, M., et al. (2017). Notch-Jagged Complex Structure Implicates a Catch Bond in Tuning Ligand Sensitivity. Science 355 (6331), 1320-1324. doi:10.1126/science.aaf9739

Ługowski, R., Kołodziejczyk, B., and Kawata, Y. (2002). Application of LaserTrapping Technique for Measuring the Three-Dimensional Distribution of Viscosity. Opt. Commun. 202 (1-3), 1-8. doi:10.1016/S0030-4018(01)01648-0

Lussi, J., Falconnet, D., Hubbell, J., Textor, M., and Csucs, G. (2006). Pattern Stability under Cell Culture Conditions-A Comparative Study of Patterning Methods Based on PLL-G-PEG Background Passivation. Biomaterials 27 (12), 2534-2541. doi:10.1016/j.biomaterials.2005.11.027

Luxton, G. W. G., Gomes, E. R., Folker, E. S., Vintinner, E., and Gundersen, G. G. (2010). Linear Arrays of Nuclear Envelope Proteins Harness Retrograde Actin Flow for Nuclear Movement. Science 329 (5994), 956-959. doi:10.1126/science. 1189072

Ma, L., Rajshekhar, G., Wang, R., Bhaduri, B., Sridharan, S., Mir, M., et al. (2016). Phase Correlation Imaging of Unlabeled Cell Dynamics. Scientific Rep. 6 (1), 32702. doi: $10.1038 /$ srep 32702

Mack, J. J., Mosqueiro, T. S., Archer, B. J., Jones, W. M., Sunshine, H., Faas, G. C., et al. (2017). NOTCH1 Is a Mechanosensor in Adult Arteries. Nat. Commun. 8 (1), 1620. doi:10.1038/s41467-017-01741-8

Maekawa, M., Ishizaki, T., Boku, S., Watanabe, N., Fujita, A., Iwamatsu, A., et al. (1999). Signaling from Rho to the Actin Cytoskeleton through Protein Kinases ROCK and LIM-Kinase. Science 285 (5429), 895-898. doi:10.1126/science.285. 5429.895

Mahaffy, R. E., Shih, C. K., MacKintosh, F. C., and Käs, J. (2000). Scanning ProbeBased Frequency-dependent Microrheology of Polymer Gels and Biological Cells. Phys. Rev. Lett. 85 (4), 880-883. doi:10.1103/PhysRevLett.85.880

Mak, A. F. (1986). The Apparent Viscoelastic Behavior of Articular Cartilage-The Contributions from the Intrinsic Matrix Viscoelasticity and Interstitial Fluid Flows. J. Biomechanical Eng. 108 (2), 123-130. doi:10.1115/1.3138591

Malinverno, C., Corallino, S., Giavazzi, F., Bergert, M., Li, Q., Leoni, M., et al. (2017). Endocytic Reawakening of Motility in Jammed Epithelia. Nat. Mater. 16 (5), 587-596. doi:10.1038/nmat4848

Mandal, K., Raz-Ben Aroush, D., Graber, Z. T., Wu, B., Park, C. Y., Fredberg, J. J., et al. (2019). Soft Hyaluronic Gels Promote Cell Spreading, Stress Fibers, Focal Adhesion, and Membrane Tension by Phosphoinositide Signaling, Not Traction Force. ACS Nano 13 (1), 203-214. doi:10.1021/acsnano.8b05286

Marcus, W. D., and Hochmuth, R. M. (2002). Experimental Studies of Membrane Tethers Formed from Human Neutrophils. Ann. Biomed. Eng. 30 (10), 1273-1280. doi:10.1114/1.1528614

Marée, A. F. M., Grieneisen, V. A., and Edelstein-Keshet, L. (2012). How Cells Integrate Complex Stimuli: The Effect of Feedback from Phosphoinositides and Cell Shape on Cell Polarization and Motility. PLoS Comput. Biol. 8 (3), e1002402. doi:10.1371/journal.pcbi.1002402
Marée, A. F. M., Jilkine, A., Dawes, A., Grieneisen, V. A., and Edelstein-Keshet, L. (2006). Polarization and Movement of Keratocytes: A Multiscale Modelling Approach. Bull. Math. Biol. 68 (5), 1169-1211. doi:10.1007/s11538-006-9131-7

Marie, R., Dahlin, A. B., Tegenfeldt, J. O., and Höök, F. (2007). Generic Surface Modification Strategy for Sensing Applications Based on $\mathrm{Au} / \mathrm{SiO}_{2}$ Nanostructures. Biointerphases 2 (1), 49-55. doi:10.1116/1.2717926

Marion, S., Guillen, N., Bacri, J.-C., and Wilhelm, C. (2005). Acto-myosin Cytoskeleton Dependent Viscosity and Shear-Thinning Behavior of the Amoeba Cytoplasm. Eur. Biophys. J. 34 (3), 262-272. doi:10.1007/s00249004-0449-5

Marmottant, P., Mgharbel, A., Kafer, J., Audren, B., Rieu, J.-P., Vial, J.-C., et al. (2009). The Role of Fluctuations and Stress on the Effective Viscosity of Cell Aggregates. Proc. Natl. Acad. Sci. 106 (41), 17271-17275. doi:10.1073/pnas. 0902085106

Maroudas, A., Wachtel, E., Grushko, G., Katz, E. P., and Weinberg, P. (1991). The Effect of Osmotic and Mechanical Pressures on Water Partitioning in Articular Cartilage. Biochim. Biophys. Acta (Bba) - Gen. Subjects 1073 (2), 285-294. doi:10.1016/0304-4165(91)90133-2

Martino, F., Perestrelo, A. R., Vinarský, V., Pagliari, S., and Forte, G. (2018). Cellular Mechanotransduction: From Tension to Function. Front. Physiol. 9, 824. doi:10.3389/fphys.2018.00824

Maruthamuthu, V., Sabass, B., Schwarz, U. S., and Gardel, M. L. (2011). Cell-ECM Traction Force Modulates Endogenous Tension at Cell-Cell Contacts. Proc. Natl. Acad. Sci. 108 (12), 4708-4713. doi:10.1073/pnas.1011123108

Mason, T. G., and Weitz, D. A. (1995). Optical Measurements of Frequencydependent Linear Viscoelastic Moduli of Complex Fluids. Phys. Rev. Lett. 74 (7), 1250-1253. doi:10.1103/PhysRevLett.74.1250

Mastro, A. M., and Keith, A. D. (1984). Diffusion in the Aqueous Compartment. J. Cel Biol. 99 (1), 180s-187s. doi:10.1083/jcb.99.1.180s

Masumura, T., Yamamoto, K., Shimizu, N., Obi, S., and Ando, J. (2009). Shear Stress Increases Expression of the Arterial Endothelial Marker EphrinB2 in Murine ES Cells via the VEGF-Notch Signaling Pathways. Arteriosclerosis, Thromb. Vasc. Biol. 29 (12), 2125-2131. doi:10.1161/ATVBAHA.109.193185

Matthews, B. D., Overby, D. R., Mannix, R., and Ingber, D. E. (2006). Cellular Adaptation to Mechanical Stress: Role of Integrins, Rho, Cytoskeletal Tension and Mechanosensitive Ion Channels. J. Cel Sci. 119 (3), 508-518. doi:10.1242/ jcs. 02760

Mayor, R., and Carmona-Fontaine, C. (2010). Keeping in Touch with Contact Inhibition of Locomotion. Trends Cel Biol. 20 (6), 319-328. doi:10.1016/j.tcb. 2010.03.005

McGlynn, J. A., Wu, N., and Schultz, K. M. (2020). Multiple Particle Tracking Microrheological Characterization: Fundamentals, Emerging Techniques and Applications. J. Appl. Phys. 127 (20), 201101. doi:10.1063/5.0006122

Mehta, V., Pang, K.-L., Rozbesky, D., Nather, K., Keen, A., Lachowski, D., et al. (2020). The Guidance Receptor Plexin D1 Is a Mechanosensor in Endothelial Cells. Nature 578 (7794), 290-295. doi:10.1038/s41586-020-1979-4

Melcer, S., Hezroni, H., Rand, E., Nissim-Rafinia, M., Skoultchi, A., Stewart, C. L., et al. (2012). Histone Modifications and Lamin A Regulate Chromatin Protein Dynamics in Early Embryonic Stem Cell Differentiation. Nat. Commun. 3 (1), 910. doi:10.1038/ncomms1915

Meloty-Kapella, L., Shergill, B., Kuon, J., Botvinick, E., and Weinmaster, G. (2012). Notch Ligand Endocytosis Generates Mechanical Pulling Force Dependent on Dynamin, Epsins, and Actin. Developmental Cel 22 (6), 1299-1312. doi:10. 1016/j.devcel.2012.04.005

Meng, W., and Takeichi, M. (2009). Adherens Junction: Molecular Architecture and Regulation. Cold Spring Harbor Perspect. Biol. 1 (6), a002899. doi:10.1101/ cshperspect.a002899

Messica, Y., Laser-Azogui, A., Volberg, T., Elisha, Y., Lysakovskaia, K., Eils, R., et al. (2017). The Role of Vimentin in Regulating Cell Invasive Migration in Dense Cultures of Breast Carcinoma Cells. Nano Lett. 17 (11), 6941-6948. doi:10. 1021/acs.nanolett.7b03358

Michel, R., Lussi, J. W., Csucs, G., Reviakine, I., Danuser, G., Ketterer, B., et al. (2002). Selective Molecular Assembly Patterning: A New Approach to Microand Nanochemical Patterning of Surfaces for Biological Applications. Langmuir 18 (8), 3281-3287. doi:10.1021/la011715y

Mierke, C. T., Fischer, T., Puder, S., Kunschmann, T., Soetje, B., and Ziegler, W. H. (2017). Focal Adhesion Kinase Activity Is Required for Actomyosin 
Contractility-Based Invasion of Cells into Dense 3D Matrices. Scientific Rep. 7 (1), 42780. doi:10.1038/srep42780

Mierke, C. T., Frey, B., Fellner, M., Herrmann, M., and Fabry, B. (2011). Integrin a $5 \beta 1$ Facilitates Cancer Cell Invasion through Enhanced Contractile Forces. J. Cel Sci. 124 (3), 369-383. doi:10.1242/jcs.071985

Mierke, C. T. (2020). Mechanical Cues Affect Migration and Invasion of Cells from Three Different Directions. Front. Cel Developmental Biol. 8, 583226. doi:10. 3389/fcell.2020.583226

Mierke, C. T., Puder, S., Aermes, C., Fischer, T., and Kunschmann, T. (2020). Effect of PAK Inhibition on Cell Mechanics Depends on Racl. Front. Cel Developmental Biol. 8, 13. doi:10.3389/fcell.2020.00013

Mierke, C. T. (2014). The Fundamental Role of Mechanical Properties in the Progression of Cancer Disease and Inflammation. Rep. Prog. Phys. 77 (7), 076602. doi:10.1088/0034-4885/77/7/076602

Mierke, C. T. (2019). The Matrix Environmental and Cell Mechanical Properties Regulate Cell Migration and Contribute to the Invasive Phenotype of Cancer Cells. Rep. Prog. Phys. 82 (6), 064602. doi:10.1088/1361-6633/ab1628

Mierke, C. T., Zitterbart, D. P., Kollmannsberger, P., Raupach, C., SchlötzerSchrehardt, U., Goecke, T. W., et al. (2008). Breakdown of the Endothelial Barrier Function in Tumor Cell Transmigration. Biophysical J. 94 (7), 2832-2846. doi:10.1529/biophysj.107.113613

Miller, K. (2000). Constitutive Modelling of Abdominal Organs. J. Biomech. 33 (3), 367-373. doi:10.1016/S0021-9290(99)00196-7

Mir, M., Bergamaschi, A., Katzenellenbogen, B. S., and Popescu, G. (2014). Highly Sensitive Quantitative Imaging for Monitoring Single Cancer Cell Growth Kinetics and Drug Response. PLoS ONE 9 (2), e89000. doi:10.1371/journal. pone. 0089000

Mir, M., Wang, Z., Shen, Z., Bednarz, M., Bashir, R., Golding, I., et al. (2011). Optical Measurement of Cycle-dependent Cell Growth. Proc. Natl. Acad. Sci. 108 (32), 13124-13129. doi:10.1073/pnas.1100506108

Mitchison, T. J., Charras, G. T., and Mahadevan, L. (2008). Implications of a Poroelastic Cytoplasm for the Dynamics of Animal Cell Shape. Semin. Cel Developmental Biol. 19 (3), 215-223. doi:10.1016/j.semcdb.2008.01.008

Mitrossilis, D., Fouchard, J., Pereira, D., Postic, F., Richert, A., Saint-Jean, M., et al. (2010). Real-time Single-Cell Response to Stiffness. Proc. Natl. Acad. Sci. 107 (38), 16518-16523. doi:10.1073/pnas.1007940107

Moeendarbary, E., and Harris, A. R. (2014). Cell Mechanics: Principles, Practices, and Prospects: Cell Mechanics. Wiley Interdiscip. Rev. Syst. Biol. Med. 6 (5), 371-388. doi:10.1002/wsbm.1275

Moeendarbary, E., Valon, L., Fritzsche, M., Harris, A. R., Moulding, D. A., Thrasher, A. J., et al. (2013). The Cytoplasm of Living Cells Behaves as a Poroelastic Material. Nat. Mater. 12 (3), 253-261. doi:10.1038/nmat3517

Mollaeian, K., Liu, Y., Bi, S., and Ren, J. (2018). Atomic Force Microscopy Study Revealed Velocity-Dependence and Nonlinearity of Nanoscale Poroelasticity of Eukaryotic Cells. J. Mech. Behav. Biomed. Mater. 78, 65-73. doi:10.1016/j. jmbbm.2017.11.001

Monaghan-Benson, E., Wittchen, E. S., Doerschuk, C. M., and Burridge, K. (2018). A Rnd3/p190RhoGAP Pathway Regulates RhoA Activity in Idiopathic Pulmonary Fibrosis Fibroblasts. Mol. Biol. Cel 29 (18), 2165-2175. doi:10. 1091/mbc.E17-11-0642

Mongera, A., Rowghanian, P., Gustafson, H. J., Shelton, E., Kealhofer, D. A., Carn, E. K., et al. (2018). A Fluid-To-Solid Jamming Transition Underlies Vertebrate Body axis Elongation. Nature 561 (7723), 401-405. doi:10.1038/s41586-0180479-2

Montell, D. J., Yoon, W. H., and Starz-Gaiano, M. (2012). Group Choreography: Mechanisms Orchestrating the Collective Movement of Border Cells. Nat. Rev. Mol. Cel Biol. 13 (10), 631-645. doi:10.1038/nrm3433

Moore, S. W., Roca-Cusachs, P., and Sheetz, M. P. (2010). Stretchy Proteins on Stretchy Substrates: The Important Elements of Integrin-Mediated Rigidity Sensing. Developmental Cel 19 (2), 194-206. doi:10.1016/j.devcel.2010.07.018

Morsut, L., Roybal, K. T., Xiong, X., Gordley, R. M., Coyle, S. M., Thomson, M., et al. (2016). Engineering Customized Cell Sensing and Response Behaviors Using Synthetic Notch Receptors. Cell 164 (4), 780-791. doi:10.1016/j.cell.2016. 01.012

Mow, V. C., Ratcliffe, A., and Robin Poole, A. (1992). Cartilage and Diarthrodial Joints as Paradigms for Hierarchical Materials and Structures. Biomaterials 13 (2), 67-97. doi:10.1016/0142-9612(92)90001-5
Mseka, T., Bamburg, J. R., and Cramer, L. P. (2007). ADF/cofilin Family Proteins Control Formation of Oriented Actin-Filament Bundles in the Cell Body to Trigger Fibroblast Polarization. J. Cel Sci. 120 (24), 4332-4344. doi:10.1242/jcs. 017640

Mueller, J., Szep, G., Nemethova, M., de Vries, I., Lieber, A. D., Winkler, C., et al. (2017). Load Adaptation of Lamellipodial Actin Networks. Cell 171 (1), 188-200. doi:10.1016/j.cell.2017.07.051

Mueller, S. (2010). Increased Liver Stiffness in Alcoholic Liver Disease: Differentiating Fibrosis from Steatohepatitis. World J. Gastroenterol. 16 (8), 966. doi:10.3748/wjg.v16.i8.966

Murphy-Ullrich, J. E. (2001). The De-adhesive Activity of Matricellular Proteins: Is Intermediate Cell Adhesion an Adaptive State? J. Clin. Invest. 107 (7), 785-790. doi:10.1172/JCI12609

Murrell, M., Oakes, P. W., Lenz, M., and Gardel, M. L. (2015). Forcing Cells into Shape: The Mechanics of Actomyosin Contractility. Nat. Rev. Mol. Cel Biol. 16 (8), 486-498. doi:10.1038/nrm4012

Musaev, O. R., Scott, P., Wrobel, J. M., Wolf, J. A., and Kruger, M. B. (2011). UV Laser Ablation of Parylene Films from Gold Substrates. J. Mater. Sci. 46 (1), 183-187. doi:10.1007/s10853-010-4906-5

Muthupillai, R., Lomas, D., Rossman, P., Greenleaf, J., Manduca, A., and Ehman, R. (1995). Magnetic Resonance Elastography by Direct Visualization of Propagating Acoustic Strain Waves. Science 269 (5232), 1854-1857. doi:10. 1126/science.7569924

Nagayama, M., Haga, H., and Kawabata, K. (2001). Drastic Change of Local Stiffness Distribution Correlating to Cell Migration in Living Fibroblasts. Cell Motil. Cytoskeleton 50 (4), 173-179. doi:10.1002/cm.10008

Nakaya, Y., and Sheng, G. (2008). Epithelial to Mesenchymal Transition during Gastrulation: An Embryological View: EMT in Gastrulation. Dev. Growth Differ. 50 (9), 755-766. doi:10.1111/j.1440-169X.2008.01070.x

Nawaz, S., Sánchez, P., Bodensiek, K., Li, S., Simons, M., and Schaap, I. A. T. (2012). Cell Visco-Elasticity Measured with AFM and Optical Trapping at Submicrometer Deformations. PLoS ONE 7 (9), e45297. doi:10.1371/journal. pone. 0045297

Neal, B. L., Asthagiri, D., and Lenhoff, A. M. (1998). Molecular Origins of Osmotic Second Virial Coefficients of Proteins. Biophysical J. 75 (5), 2469-2477. doi:10. 1016/S0006-3495(98)77691-X

Neel, B. L., Nisler, C. R., Walujkar, S., Araya-Secchi, R., and Sotomayor, M. (2021). Elastic Versus Brittle Mechanical Responses Predicted For Dimeric Cadherin Complexes [Preprint]. Biophysics. doi:10.1101/2021.07.29.454067

Nelson, C. M., VanDuijn, M. M., Inman, J. L., Fletcher, D. A., and Bissell, M. J. (2006). Tissue Geometry Determines Sites of Mammary Branching Morphogenesis in Organotypic Cultures. Science 314 (5797), 298-300. doi:10.1126/science.1131000

Nematbakhsh, Y., Pang, K. T., and Lim, C. T. (2017). Correlating the Viscoelasticity of Breast Cancer Cells with Their Malignancy. Convergent Sci. Phys. Oncol. 3 (3), 034003. doi:10.1088/2057-1739/aa7ffb

Neto, F., Klaus-Bergmann, A., Ong, Y. T., Alt, S., Vion, A.-C., Szymborska, A., et al. (2018). YAP and TAZ Regulate Adherens junction Dynamics and Endothelial Cell Distribution during Vascular Development. ELife 7, e31037. doi:10.7554/ eLife. 31037

Nguyen, T. L., Polanco, E. R., Patananan, A. N., Zangle, T. A., and Teitell, M. A. (2020). Cell Viscoelasticity Is Linked to Fluctuations in Cell Biomass Distributions. Scientific Rep. 10 (1), 7403. doi:10.1038/s41598-020-64259-y

Nia, H. T., Bozchalooi, I. S., Li, Y., Han, L., Hung, H.-H., Frank, E., et al. (2013). High-Bandwidth AFM-Based Rheology Reveals that Cartilage Is Most Sensitive to High Loading Rates at Early Stages of Impairment. Biophysical J. 104 (7), 1529-1537. doi:10.1016/j.bpj.2013.02.048

Nia, H. T., Han, L., Li, Y., Ortiz, C., and Grodzinsky, A. (2011). Poroelasticity of Cartilage at the Nanoscale. Biophysical J. 101 (9), 2304-2313. doi:10.1016/j.bpj. 2011.09.011

Nia, H. T., Han, L., Soltani Bozchalooi, I., Roughley, P., Youcef-Toumi, K., Grodzinsky, A. J., et al. (2015). Aggrecan Nanoscale Solid-Fluid Interactions Are a Primary Determinant of Cartilage Dynamic Mechanical Properties. ACS Nano 9 (3), 2614-2625. doi:10.1021/nn5062707

Nicolle, S., Noguer, L., and Palierne, J.-F. (2013). Shear Mechanical Properties of the Porcine Pancreas: Experiment and Analytical Modelling. J. Mech. Behav. Biomed. Mater. 26, 90-97. doi:10.1016/j.jmbbm.2013.05.029 
Nicolle, S., Vezin, P., and Palierne, J.-F. (2010). A Strain-Hardening Bi-power Law for the Nonlinear Behaviour of Biological Soft Tissues. J. Biomech. 43 (5), 927-932. doi:10.1016/j.jbiomech.2009.11.002

Nicoludis, J. M., Vogt, B. E., Green, A. G., Schärfe, C. P., Marks, D. S., and Gaudet, R. (2016). Antiparallel Protocadherin Homodimers Use Distinct Affinity- and Specificity-Mediating Regions in Cadherin Repeats 1-4. ELife 5, e18449. doi:10. 7554/eLife.18449

Nieto, M. A., Huang, R. Y.-J., Jackson, R. A., and Thiery, J. P. (2016). EMT: 2016. Cell 166 (1), 21-45. doi:10.1016/j.cell.2016.06.028

Nieto, M. A. (2011). The Ins and Outs of the Epithelial to Mesenchymal Transition in Health and Disease. Annu. Rev. Cel Developmental Biol. 27 (1), 347-376. doi:10.1146/annurev-cellbio-092910-154036

Nnetu, K. D., Knorr, M., Käs, J., and Zink, M. (2012). The Impact of Jamming on Boundaries of Collectively Moving Weak-Interacting Cells. New J. Phys. 14 (11), 115012. doi:10.1088/1367-2630/14/11/115012

Nnetu, K. D., Knorr, M., Pawlizak, S., Fuhs, T., and Käs, J. A. (2013). Slow and Anomalous Dynamics of an MCF-10A Epithelial Cell Monolayer. Soft Matter 9 (39), 9335. doi:10.1039/c3sm50806d

Ocaña, O. H., Córcoles, R., Fabra, Á., Moreno-Bueno, G., Acloque, H., Vega, S., et al. (2012). Metastatic Colonization Requires the Repression of the EpithelialMesenchymal Transition Inducer Prrx1. Cancer Cell 22 (6), 709-724. doi:10. 1016/j.ccr.2012.10.012

Onken, M. D., Mooren, O. L., Mukherjee, S., Shahan, S. T., Li, J., and Cooper, J. A. (2014). Endothelial Monolayers and Transendothelial Migration Depend on Mechanical Properties of the Substrate: Substrate Stiffness Affects Transendothelial Migration. Cytoskeleton 71 (12), 695-706. doi:10.1002/cm. 21203

Orr, A. W., Helmke, B. P., Blackman, B. R., and Schwartz, M. A. (2006). Mechanisms of Mechanotransduction. Developmental Cel 10 (1), 11-20. doi:10.1016/j.devcel.2005.12.006

Oswald, L., Grosser, S., Smith, D. M., and Käs, J. A. (2017). Jamming Transitions in Cancer. J. Phys. D: Appl. Phys. 50 (48), 483001. doi:10.1088/1361-6463/aa8e83

Otsuka, S., and Ellenberg, J. (2018). Mechanisms of Nuclear Pore Complex Assembly - Two Different Ways of Building One Molecular Machine. FEBS Lett. 592 (4), 475-488. doi:10.1002/1873-3468.12905

Owen, L. M., Bax, N. A., Weis, W. I., and Dunn, A. R. (2020). The C-Terminal Actin Binding Domain of Talin Forms an Asymmetric Catch Bond with F-Actin [Preprint]. Biophysics. doi:10.1101/2020.09.01.276568

Pajic-Lijakovic, I., and Milivojevic, M. (2019a). Functional Epithelium Remodeling in Response to Applied Stress under In Vitro Conditions. Appl. Bionics Biomech. 2019, 1-13. doi:10.1155/2019/4892709

Pajic-Lijakovic, I., and Milivojevic, M. (2019b). Long-time Viscoelasticity of Multicellular Surfaces Caused by Collective Cell Migration - Multi-Scale Modeling Considerations. Semin. Cel Developmental Biol. 93, 87-96. doi:10. 1016/j.semcdb.2018.08.002

Pajic-Lijakovic, I., and Milivojevic, M. (2020). Mechanical Oscillations in 2D Collective Cell Migration: The Elastic Turbulence. Front. Phys. 8, 585681. doi:10.3389/fphy.2020.585681

Pajic-Lijakovic, I., and Milivojevic, M. (2021a). Multiscale Nature of Cell Rearrangement Caused by Collective Cell Migration. Eur. Biophys. J. 50 (1), 1-14. doi:10.1007/s00249-021-01496-7

Pajic-Lijakovic, I., and Milivojevic, M. (2021b). Viscoelasticity And Cell Jamming State Transition [Preprint]. Developmental Biol. doi:10.1101/2021.03.19. 436195

Pajic-Lijakovic, I., and Milivojevic, M. (2017). Viscoelasticity of Multicellular Surfaces. J. Biomech. 60, 1-8. doi:10.1016/j.jbiomech.2017.06.035

Paluch, E. K., Nelson, C. M., Biais, N., Fabry, B., Moeller, J., Pruitt, B. L., et al. (2015). Mechanotransduction: Use the Force(s). BMC Biol. 13 (1), 47. doi:10. 1186/s12915-015-0150-4

Pan, Y., Heemskerk, I., Ibar, C., Shraiman, B. I., and Irvine, K. D. (2016). Differential Growth Triggers Mechanical Feedback that Elevates Hippo Signaling. Proc. Natl. Acad. Sci. 113 (45), E6974-E6983. doi:10.1073/pnas.1615012113

Panciera, T., Azzolin, L., Cordenonsi, M., and Piccolo, S. (2017). Mechanobiology of YAP and TAZ in Physiology and Disease. Nat. Rev. Mol. Cel Biol. 18 (12), 758-770. doi:10.1038/nrm.2017.87

Pandya, P., Orgaz, J. L., and Sanz-Moreno, V. (2017). Actomyosin Contractility and Collective Migration: May the Force Be with You. Curr. Opin. Cel Biol. 48, 87-96. doi:10.1016/j.ceb.2017.06.006
Panorchan, P., Schafer, B. W., Wirtz, D., and Tseng, Y. (2004). Nuclear Envelope Breakdown Requires Overcoming the Mechanical Integrity of the Nuclear Lamina. J. Biol. Chem. 279 (42), 43462-43467. doi:10.1074/jbc.M402474200

Papagrigoriou, E., Gingras, A. R., Barsukov, I. L., Bate, N., Fillingham, I. J., Patel, B., et al. (2004). Activation of a Vinculin-Binding Site in the Talin Rod Involves Rearrangement of a Five-helix Bundle. EMBO J. 23 (15), 2942-2951. doi:10. 1038/sj.emboj.7600285

Park, J.-A., Atia, L., Mitchel, J. A., Fredberg, J. J., and Butler, J. P. (2016). Collective Migration and Cell Jamming in Asthma, Cancer and Development. J. Cel Sci. jcs, 187922. doi:10.1242/jcs.187922

Park, K.-S., Wells, J. M., Zorn, A. M., Wert, S. E., Laubach, V. E., Fernandez, L. G., et al. (2006). Transdifferentiation of Ciliated Cells during Repair of the Respiratory Epithelium. Am. J. Respir. Cel Mol. Biol. 34 (2), 151-157. doi:10.1165/rcmb.2005-0332OC

Park, Y., Best, C. A., Badizadegan, K., Dasari, R. R., Feld, M. S., Kuriabova, T., et al. (2010). Measurement of Red Blood Cell Mechanics during Morphological Changes. Proc. Natl. Acad. Sci. 107 (15), 6731-6736. doi:10.1073/pnas. 0909533107

Pasapera, A. M., Schneider, I. C., Rericha, E., Schlaepfer, D. D., and Waterman, C. M. (2010). Myosin II Activity Regulates Vinculin Recruitment to Focal Adhesions through FAK-Mediated Paxillin Phosphorylation. J. Cel Biol. 188 (6), 877-890. doi:10.1083/jcb.200906012

Pastor-Pareja, J. C., Grawe, F., Martı'n-Blanco, E., and Garcı'a-Bellido, A. (2004). Invasive Cell Behavior during Drosophila Imaginal Disc Eversion Is Mediated by the JNK Signaling Cascade. Developmental Cel 7 (3), 387-399. doi:10.1016/j. devcel.2004.07.022

Paszek, M. J., and Weaver, V. M. (2004). The Tension Mounts: Mechanics Meets Morphogenesis and Malignancy. J. Mammary Gland Biol. Neoplasia 9 (4), 325-342. doi:10.1007/s10911-004-1404-x

Paszek, M. J., Zahir, N., Johnson, K. R., Lakins, J. N., Rozenberg, G. I., Gefen, A., et al. (2005). Tensional Homeostasis and the Malignant Phenotype. Cancer Cell 8 (3), 241-254. doi:10.1016/j.ccr.2005.08.010

Paul, R., Heil, P., Spatz, J. P., and Schwarz, U. S. (2008). Propagation of Mechanical Stress through the Actin Cytoskeleton toward Focal Adhesions: Model and Experiment. Biophysical J. 94 (4), 1470-1482. doi:10.1529/biophysj.107.108688

Pavillon, N., Kühn, J., Moratal, C., Jourdain, P., Depeursinge, C., Magistretti, P. J., et al. (2012). Early Cell Death Detection with Digital Holographic Microscopy. PLoS ONE 7 (1), e30912. doi:10.1371/journal.pone.0030912

Pegoraro, A. F., Janmey, P., and Weitz, D. A. (2017). Mechanical Properties of the Cytoskeleton and Cells. Cold Spring Harbor Perspect. Biol. 9 (11), a022038. doi:10.1101/cshperspect.a022038

Pelham, R. J., and Wang, Y. -l. (1997). Cell Locomotion and Focal Adhesions Are Regulated by Substrate Flexibility. Proc. Natl. Acad. Sci. 94 (25), 13661-13665. doi:10.1073/pnas.94.25.13661

Perl, A.-K., Wilgenbus, P., Dahl, U., Semb, H., and Christofori, G. (1998). A Causal Role for E-Cadherin in the Transition from Adenoma to Carcinoma. Nature 392 (6672), 190-193. doi:10.1038/32433

Perrinez, P. R., Kennedy, F. E., Van Houten, E. E. W., Weaver, J. B., and Paulsen, K. D. (2009). Modeling of Soft Poroelastic Tissue in Time-Harmonic MR Elastography. IEEE Trans. Biomed. Eng. 56 (3), 598-608. doi:10.1109/ TBME.2008.2009928

Petersen, N. O., McConnaughey, W. B., and Elson, E. L. (1982). Dependence of Locally Measured Cellular Deformability on Position on the Cell, Temperature, and Cytochalasin B. Proc. Natl. Acad. Sci. 79 (17), 5327-5331. doi:10.1073/pnas. 79.17.5327

Petridou, N. I., Corominas-Murtra, B., Heisenberg, C.-P., and Hannezo, E. (2021). Rigidity Percolation Uncovers a Structural Basis for Embryonic Tissue Phase Transitions. Cell 184 (7), 1914-1928. doi:10.1016/j.cell.2021.02.017

Petridou, N. I., Grigolon, S., Salbreux, G., Hannezo, E., and Heisenberg, C.-P. (2019). Fluidization-mediated Tissue Spreading by Mitotic Cell Rounding and Non-canonical Wnt Signalling. Nat. Cel Biol. 21 (2), 169-178. doi:10.1038/ s41556-018-0247-4

Petridou, N. I., and Heisenberg, C. (2019). Tissue Rheology in Embryonic Organization. EMBO J. 38 (20). doi:10.15252/embj.2019102497

Petridou, N. I., Spiró, Z., and Heisenberg, C.-P. (2017). Multiscale Force Sensing in Development. Nat. Cel Biol. 19 (6), 581-588. doi:10.1038/ncb3524

Petrie, R. J., and Yamada, K. M. (2012). At the Leading Edge of Three-Dimensional Cell Migration. J. Cel Sci. jcs, 093732. doi:10.1242/jcs.093732 
Petrovic, J., Formosa-Jordan, P., Luna-Escalante, J. C., Abelló, G., Ibañes, M., Neves, J., et al. (2014). Ligand-dependent Notch Signaling Strength Orchestrates Lateral Induction and Lateral Inhibition in the Developing Inner Ear. Development 141 (11), 2313-2324. doi:10.1242/dev.108100

Peyton, S. R., Kalcioglu, Z. I., Cohen, J. C., Runkle, A. P., Van Vliet, K. J., Lauffenburger, D. A., et al. (2011). Marrow-Derived Stem Cell Motility in 3D Synthetic Scaffold Is Governed by Geometry along with Adhesivity and Stiffness. Biotechnol. Bioeng. 108 (5), 1181-1193. doi:10.1002/bit.23027

Pirzer, T., and Hugel, T. (2009). Atomic Force Microscopy spring Constant Determination in Viscous Liquids. Rev. Scientific Instr. 80 (3), 035110. doi:10.1063/1.3100258

Pogoda, K., Chin, L., Georges, P. C., Byfield, F. J., Bucki, R., Kim, R., et al. (2014). Compression Stiffening of Brain and its Effect on Mechanosensing by Glioma Cells. New J. Phys. 16 (7), 075002. doi:10.1088/1367-2630/16/7/075002

Poh, Y.-C., Chen, J., Hong, Y., Yi, H., Zhang, S., Chen, J., et al. (2014). Generation of Organized Germ Layers from a Single Mouse Embryonic Stem Cell. Nat. Commun. 5 (1), 4000. doi:10.1038/ncomms5000

Polacheck, W. J., Kutys, M. L., Yang, J., Eyckmans, J., Wu, Y., Vasavada, H., et al. (2017). A Non-canonical Notch Complex Regulates Adherens Junctions and Vascular Barrier Function. Nature 552 (7684), 258-262. doi:10.1038/ nature24998

Pontes, B., Monzo, P., Gole, L., Le Roux, A.-L., Kosmalska, A. J., Tam, Z. Y., et al. (2017). Membrane Tension Controls Adhesion Positioning at the Leading Edge of Cells. J. Cel Biol. 216 (9), 2959-2977. doi:10.1083/jcb.201611117

Popescu, G., Park, Y., Choi, W., Dasari, R. R., Feld, M. S., and Badizadegan, K. (2008). Imaging Red Blood Cell Dynamics by Quantitative Phase Microscopy. Blood Cell Mol. Dis. 41 (1), 10-16. doi:10.1016/j.bcmd.2008.01.010

Popescu, G., and Park, Y. (2015). Special Section Guest Editorial:Quantitative Phase Imaging in Biomedicine. J. Biomed. Opt. 20 (11), 111201. doi:10.1117/1. JBO.20.11.111201

Prabhakarpandian, B., Shen, M.-C., Nichols, J. B., Mills, I. R., SidorykWegrzynowicz, M., Aschner, M., et al. (2013). SyM-BBB: A Microfluidic Blood Brain Barrier Model. Lab. A Chip 13 (6), 1093. doi:10.1039/c2lc41208j

Prescott, D. M., Myerson, D., and Wallace, J. (1972). Enucleation of Mammalian Cells with Cytochalasin B. Exp. Cel Res. 71 (2), 480-485. doi:10.1016/00144827(72)90322-9

Pritchard, R. H., Shery Huang, Y. Y., and Terentjev, E. M. (2014). Mechanics of Biological Networks: From the Cell Cytoskeleton to Connective Tissue. Soft Matter 10 (12), 1864. doi:10.1039/c3sm52769g

Provenzano, P. P., Eliceiri, K. W., Campbell, J. M., Inman, D. R., White, J. G., and Keely, P. J. (2006). Collagen Reorganization at the Tumor-Stromal Interface Facilitates Local Invasion. BMC Med. 4 (1), 38. doi:10.1186/1741-7015-4-38

Provenzano, P. P., Inman, D. R., Eliceiri, K. W., and Keely, P. J. (2009). Matrix Density-Induced Mechanoregulation of Breast Cell Phenotype, Signaling and Gene Expression through a FAK-ERK Linkage. Oncogene 28 (49), 4326-4343. doi:10.1038/onc.2009.299

Puisieux, A., Brabletz, T., and Caramel, J. (2014). Oncogenic Roles of EMTInducing Transcription Factors. Nat. Cel Biol. 16 (6), 488-494. doi:10.1038/ ncb2976

Qian, H. (2000). Single-Particle Tracking: Brownian Dynamics of Viscoelastic Materials. Biophysical J. 79 (1), 137-143. doi:10.1016/S0006-3495(00)76278-3

Raab, M., Gentili, M., de Belly, H., Thiam, H.-R., Vargas, P., Jimenez, A. J., et al. (2016). ESCRT III Repairs Nuclear Envelope Ruptures during Cell Migration to Limit DNA Damage and Cell Death. Science 352 (6283), 359-362. doi:10.1126/ science.aad7611

Radisky, D. C., Levy, D. D., Littlepage, L. E., Liu, H., Nelson, C. M., Fata, J. E., et al. (2005). Rac1b and Reactive Oxygen Species Mediate MMP-3-Induced EMT and Genomic Instability. Nature 436 (7047), 123-127. doi:10.1038/nature03688

Rajgor, D., and Shanahan, C. M. (2013). Nesprins: From the Nuclear Envelope and beyond. Expert Rev. Mol. Med. 15, e5. doi:10.1017/erm.2013.6

Rao, M. V., Chu, P.-H., Hahn, K. M., and Zaidel-Bar, R. (2013). An Optogenetic Tool for the Activation of Endogenous Diaphanous-Related Formins Induces Thickening of Stress Fibers without an Increase in Contractility: PhotoActivation of Diaphanous-Related Formins. Cytoskeleton 70 (7), 394-407. doi: $10.1002 / \mathrm{cm} .21115$

Raucher, D., and Sheetz, M. P. (2000). Cell Spreading and Lamellipodial Extension Rate Is Regulated by Membrane Tension. J. Cel Biol. 148 (1), 127-136. doi:10. 1083/jcb.148.1.127
Ravasio, A., Le, A. P., Saw, T. B., Tarle, V., Ong, H. T., Bertocchi, C., et al. (2015). Regulation of Epithelial Cell Organization by Tuning Cell-Substrate Adhesion. Integr. Biol. 7 (10), 1228-1241. doi:10.1039/C5IB00196J

Rebelo, L. M., de Sousa, J. S., Mendes Filho, J., and Radmacher, M. (2013). Comparison of the Viscoelastic Properties of Cells from Different Kidney Cancer Phenotypes Measured with Atomic Force Microscopy. Nanotechnology 24 (5), 055102. doi:10.1088/0957-4484/24/5/055102

Reed, J., Chun, J., Zangle, T. A., Kalim, S., Hong, J. S., Pefley, S. E., et al. (2011). Rapid, Massively Parallel Single-Cell Drug Response Measurements via Live Cell Interferometry. Biophysical J. 101 (5), 1025-1031. doi:10.1016/j.bpj.2011.07.022

Reed, J., Troke, J. J., Schmit, J., Han, S., Teitell, M. A., and Gimzewski, J. K. (2008). Live Cell Interferometry Reveals Cellular Dynamism during Force Propagation. ACS Nano 2 (5), 841-846. doi:10.1021/nn700303f

Ren, Y., Effler, J. C., Norstrom, M., Luo, T., Firtel, R. A., Iglesias, P. A., et al. (2009). Mechanosensing through Cooperative Interactions between Myosin II and the Actin Crosslinker Cortexillin I. Curr. Biol. 19 (17), 1421-1428. doi:10.1016/j. cub.2009.07.018

Reymann, A.-C., Boujemaa-Paterski, R., Martiel, J.-L., Guerin, C., Cao, W., Chin, H. F., et al. (2012). Actin Network Architecture Can Determine Myosin Motor Activity. Science 336 (6086), 1310-1314. doi:10.1126/science.1221708

Ridley, A. J. (2003). Cell Migration: Integrating Signals from Front to Back. Science 302 (5651), 1704-1709. doi:10.1126/science.1092053

Riveline, D., Zamir, E., Balaban, N. Q., Schwarz, U. S., Ishizaki, T., Narumiya, S., et al. (2001). Focal Contacts as Mechanosensors. J. Cel Biol. 153 (6), 1175-1186. doi:10.1083/jcb.153.6.1175

Rørth, P. (2009). Collective Cell Migration. Annu. Rev. Cel Developmental Biol. 25 (1), 407-429. doi:10.1146/annurev.cellbio.042308.113231

Rørth, P. (2012). Fellow Travellers: Emergent Properties of Collective Cell Migration. EMBO Rep. 13 (11), 984-991. doi:10.1038/embor.2012.149

Rossman, K. L., Der, C. J., and Sondek, J. (2005). GEF Means Go: Turning on RHO GTPases with Guanine Nucleotide-Exchange Factors. Nat. Rev. Mol. Cel Biol. 6 (2), 167-180. doi:10.1038/nrm1587

Rothenberg, K. E., Neibart, S. S., LaCroix, A. S., and Hoffman, B. D. (2015). Controlling Cell Geometry Affects the Spatial Distribution of Load across Vinculin. Cell Mol. Bioeng. 8 (3), 364-382. doi:10.1007/s12195-015-0404-9

Rother, J., Nöding, H., Mey, I., and Janshoff, A. (2014). Atomic Force MicroscopyBased Microrheology Reveals Significant Differences in the Viscoelastic Response between Malign and Benign Cell Lines. Open Biol. 4 (5), 140046. doi:10.1098/rsob.140046

Rotsch, C., Jacobson, K., and Radmacher, M. (1999). Dimensional and Mechanical Dynamics of Active and Stable Edges in Motile Fibroblasts Investigated by Using Atomic Force Microscopy. Proc. Natl. Acad. Sci. 96 (3), 921-926. doi:10. 1073/pnas.96.3.921

Rübsam, M., Broussard, J. A., Wickström, S. A., Nekrasova, O., Green, K. J., and Niessen, C. M. (2018). Adherens Junctions and Desmosomes Coordinate Mechanics and Signaling to Orchestrate Tissue Morphogenesis and Function: An Evolutionary Perspective. Cold Spring Harbor Perspect. Biol. 10 (11), a029207. doi:10.1101/cshperspect.a029207

Ruiz, P., and Guenthert, U. (1996). The Cellular Basis of Metastasis. World J. Urol. 14 (3). doi:10.1007/BF00186893

Salbreux, G., Charras, G., and Paluch, E. (2012). Actin Cortex Mechanics and Cellular Morphogenesis. Trends Cel Biol. 22 (10), 536-545. doi:10.1016/j.tcb.2012.07.001

Salminen, A. T., Zhang, J., Madejski, G. R., Khire, T. S., Waugh, R. E., McGrath, J. L., et al. (2019). Ultrathin Dual-Scale Nano- and Microporous Membranes for Vascular Transmigration Models. Small 15 (6), 1804111. doi:10.1002/smll. 201804111

Saotome, K., Murthy, S. E., Kefauver, J. M., Whitwam, T., Patapoutian, A., and Ward, A. B. (2018). Structure of the Mechanically Activated Ion Channel Piezo1. Nature 554 (7693), 481-486. doi:10.1038/nature25453

Savla, U., Sporn, P. H. S., and Waters, C. M. (1997). Cyclic Stretch of Airway Epithelium Inhibits Prostanoid Synthesis. Am. J. Physiology-Lung Cell Mol. Physiol. 273 (5), L1013-L1019. doi:10.1152/ajplung.1997.273.5.L1013

Savla, U., and Waters, C. M. (1998). Mechanical Strain Inhibits Repair of Airway Epithelium In Vitro. Am. J. Physiology-Lung Cell Mol. Physiol. 274 (6), L883-L892. doi:10.1152/ajplung.1998.274.6.L883

Sawada, Y., Tamada, M., Dubin-Thaler, B. J., Cherniavskaya, O., Sakai, R., Tanaka, S., et al. (2006). Force Sensing by Mechanical Extension of the Src Family Kinase Substrate p130Cas. Cell 127 (5), 1015-1026. doi:10.1016/j.cell.2006.09.044 
Scarpa, E., and Mayor, R. (2016). Collective Cell Migration in Development. J. Cel Biol. 212 (2), 143-155. doi:10.1083/jcb.201508047

Schäpe, J., Prauße, S., Radmacher, M., and Stick, R. (2009). Influence of Lamin A on the Mechanical Properties of Amphibian Oocyte Nuclei Measured by Atomic Force Microscopy. Biophysical J. 96 (10), 4319-4325. doi:10.1016/j.bpj.2009. 02.048

Schiller, H. B., and Fässler, R. (2013). Mechanosensitivity and Compositional Dynamics of Cell-Matrix Adhesions. EMBO Rep. 14 (6), 509-519. doi:10.1038/ embor.2013.49

Schlesinger, S., and Meshorer, E. (2019). Open Chromatin, Epigenetic Plasticity, and Nuclear Organization in Pluripotency. Developmental Cel 48 (2), 135-150. doi:10.1016/j.devcel.2019.01.003

Schmidt, S., and Friedl, P. (2010). Interstitial Cell Migration: Integrin-dependent and Alternative Adhesion Mechanisms. Cel Tissue Res. 339 (1), 83-92. doi:10. 1007/s00441-009-0892-9

Schmoller, K. M., Lieleg, O., and Bausch, A. R. (2008). Cross-Linking Molecules Modify Composite Actin Networks Independently. Phys. Rev. Lett. 101 (11), 118102. doi:10.1103/PhysRevLett.101.118102

Schreiner, S. M., Koo, P. K., Zhao, Y., Mochrie, S. G. J., and King, M. C. (2015). The Tethering of Chromatin to the Nuclear Envelope Supports Nuclear Mechanics. Nat. Commun. 6 (1), 7159. doi:10.1038/ncomms8159

Schroyen, B., Vlassopoulos, D., Van Puyvelde, P., and Vermant, J. (2020). Bulk Rheometry at High Frequencies: A Review of Experimental Approaches. Rheologica Acta 59 (1), 1-22. doi:10.1007/s00397-019-01172-w

Schwartz, M. A., and Shattil, S. J. (2000). Signaling Networks Linking Integrins and Rho Family GTPases. Trends Biochem. Sci. 25 (8), 388-391. doi:10.1016/S09680004(00)01605-4

Scott, D. W., Tolbert, C. E., and Burridge, K. (2016). Tension on JAM-A Activates RhoA via GEF-H1 and P115 RhoGEF. Mol. Biol. Cel 27 (9), 1420-1430. doi:10. 1091/mbc.E15-12-0833

Seetharaman, S., Vianay, B., Roca, V., Farrugia, A. J., De Pascalis, C., Boëda, B., et al. (2021). Microtubules Tune Mechanosensitive Cell Responses. Nat. Mater. doi:10.1038/s41563-021-01108-x

Segerer, F. J., Thüroff, F., Piera Alberola, A., Frey, E., and Rädler, J. O. (2015). Emergence and Persistence of Collective Cell Migration on Small Circular Micropatterns. Phys. Rev. Lett. 114 (22), 228102. doi:10.1103/PhysRevLett.114.228102

Seong, J., Tajik, A., Sun, J., Guan, J.-L., Humphries, M. J., Craig, S. E., et al. (2013). Distinct Biophysical Mechanisms of Focal Adhesion Kinase Mechanoactivation by Different Extracellular Matrix Proteins. Proc. Natl. Acad. Sci. 110 (48), 19372-19377. doi:10.1073/pnas.1307405110

Serra-Picamal, X., Conte, V., Vincent, R., Anon, E., Tambe, D. T., Bazellieres, E., et al. (2012). Mechanical Waves during Tissue Expansion. Nat. Phys. 8 (8), 628-634. doi:10.1038/nphys2355

Serwane, F., Mongera, A., Rowghanian, P., Kealhofer, D. A., Lucio, A. A., Hockenbery, Z. M., et al. (2017). In Vivo quantification of Spatially Varying Mechanical Properties in Developing Tissues. Nat. Methods 14 (2), 181-186. doi:10.1038/nmeth.4101

Seugnet, L., Simpson, P., and Haenlin, M. (1997). Requirement for Dynamin during Notch Signaling inDrosophilaNeurogenesis. Developmental Biol. 192 (2), 585-598. doi:10.1006/dbio.1997.8723

Shamir, E. R., and Ewald, A. J. (2015). Adhesion in Mammary Development. Curr. Top. Developmental Biol. 112, 353-382. doi:10.1016/bs.ctdb.2014.12.001

Sharma, V., Patel, N., Kohli, N., Ravindran, N., Hook, L., Mason, C., et al. (2016). Viscoelastic, Physical, and Bio-Degradable Properties of Dermal Scaffolds and Related Cell Behaviour. Biomed. Mater. 11 (5), 055001. doi:10.1088/1748-6041/ $11 / 5 / 055001$

Shaw, G., and Bray, D. (1977). Movement and Extension of Isolated Growth Cones. Exp. Cel Res. 104 (1), 55-62. doi:10.1016/0014-4827(77)90068-4

Shimamoto, Y., Tamura, S., Masumoto, H., and Maeshima, K. (2017). Nucleosome-nucleosome Interactions via Histone Tails and Linker DNA Regulate Nuclear Rigidity. Mol. Biol. Cel 28 (11), 1580-1589. doi:10.1091/ mbc.e16-11-0783

Shimojo, H., Ohtsuka, T., and Kageyama, R. (2008). Oscillations in Notch Signaling Regulate Maintenance of Neural Progenitors. Neuron 58 (1), 52-64. doi:10. 1016/j.neuron.2008.02.014

Shook, D., and Keller, R. (2003). Mechanisms, Mechanics and Function of Epithelial-Mesenchymal Transitions in Early Development. Mech. Development 120 (11), 1351-1383. doi:10.1016/j.mod.2003.06.005
Simpson, M. J., Lo, K.-Y., and Sun, Y.-S. (2017). Quantifying the Roles of Random Motility and Directed Motility Using Advection-Diffusion Theory for a 3T3 Fibroblast Cell Migration Assay Stimulated with an Electric Field. BMC Syst. Biol. 11 (1), 39. doi:10.1186/s12918-017-0413-5

Simpson, P. T., Reis-Filho, J. S., Gale, T., and Lakhani, S. R. (2005). Molecular Evolution of Breast Cancer: Molecular Evolution of Breast Cancer. J. Pathol. 205 (2), 248-254. doi:10.1002/path.1691

Sinkus, R., Lambert, S., Abd-Elmoniem, K. Z., Morse, C., Heller, T., Guenthner, C., et al. (2018). Rheological Determinants for Simultaneous Staging of Hepatic Fibrosis and Inflammation in Patients with Chronic Liver Disease. NMR Biomed. 31 (10), e3956. doi:10.1002/nbm.3956

Sluysmans, S., Vasileva, E., Spadaro, D., Shah, J., Rouaud, F., and Citi, S. (2017). The Role of Apical Cell-Cell Junctions and Associated Cytoskeleton in Mechanotransduction: Junctions, Cytoskeleton and Mechanotransduction. Biol. Cel 109 (4), 139-161. doi:10.1111/boc.201600075

Sniadecki, N. J., Anguelouch, A., Yang, M. T., Lamb, C. M., Liu, Z., Kirschner, S. B., et al. (2007). Magnetic Microposts as an Approach to Apply Forces to Living Cells. Proc. Natl. Acad. Sci. 104 (37), 14553-14558. doi:10.1073/pnas. 0611613104

Solovei, I., Wang, A. S., Thanisch, K., Schmidt, C. S., Krebs, S., Zwerger, M., et al. (2013). LBR and Lamin A/C Sequentially Tether Peripheral Heterochromatin and Inversely Regulate Differentiation. Cell 152 (3), 584-598. doi:10.1016/j.cell. 2013.01.009

Southern, B. D., Grove, L. M., Rahaman, S. O., Abraham, S., Scheraga, R. G., Niese, K. A., et al. (2016). Matrix-driven Myosin II Mediates the Pro-fibrotic Fibroblast Phenotype. J. Biol. Chem. 291 (12), 6083-6095. doi:10.1074/jbc. M115.712380

Spangenberg, C., Lausch, E. U., Trost, T. M., Prawitt, D., May, A., Keppler, R., et al. (2006). ERBB2-Mediated Transcriptional Up-Regulation of the $\alpha 5 \beta 1$ Integrin Fibronectin Receptor Promotes Tumor Cell Survival under Adverse Conditions. Cancer Res. 66 (7), 3715-3725. doi:10.1158/0008-5472.CAN-052823

Squires, T. M., and Mason, T. G. (2010). Fluid Mechanics of Microrheology. Annu. Rev. Fluid Mech. 42 (1), 413-438. doi:10.1146/annurev-fluid-121108-145608

Stamenović, D. (2006). Two Regimes, Maybe Three? Nat. Mater. 5 (8), 597-598. doi:10.1038/nmat1700

Stamenović, D., and Wang, N. (2000). Invited Review: Engineering Approaches to Cytoskeletal Mechanics. J. Appl. Physiol. 89 (5), 2085-2090. doi:10.1152/jappl. 2000.89.5.2085

Stanley, G. J., Fassati, A., and Hoogenboom, B. W. (2017). Biomechanics of the Transport Barrier in the Nuclear Pore Complex. Semin. Cel Developmental Biol. 68, 42-51. doi:10.1016/j.semcdb.2017.05.007

Stassen, O. M. J. A., Ristori, T., and Sahlgren, C. M. (2020). Notch in Mechanotransduction - from Molecular Mechanosensitivity to Tissue Mechanostasis. J. Cel Sci. 133 (24), jcs250738. doi:10.1242/jcs.250738

Staunton, J. R., Doss, B. L., Lindsay, S., and Ros, R. (2016). Correlating Confocal Microscopy and Atomic Force Indentation Reveals Metastatic Cancer Cells Stiffen during Invasion into Collagen I Matrices. Scientific Rep. 6 (1), 19686. doi:10.1038/srep19686

Stephens, D. C., Osunsanmi, N., Sochacki, K. A., Powell, T. W., Taraska, J. W., and Harris, D. A. (2019). Spatiotemporal Organization and Protein Dynamics Involved in Regulated Exocytosis of MMP-9 in Breast Cancer Cells. J. Gen. Physiol. 151 (12), 1386-1403. doi:10.1085/jgp.201812299

Stewart, M. P., Helenius, J., Toyoda, Y., Ramanathan, S. P., Muller, D. J., and Hyman, A. A. (2011). Hydrostatic Pressure and the Actomyosin Cortex Drive Mitotic Cell Rounding. Nature 469 (7329), 226-230. doi:10.1038/nature09642

Stewart-Hutchinson, P. J., Hale, C. M., Wirtz, D., and Hodzic, D. (2008). Structural Requirements for the Assembly of LINC Complexes and Their Function in Cellular Mechanical Stiffness. Exp. Cel Res. 314 (8), 1892-1905. doi:10.1016/j. yexcr.2008.02.022

Storm, C., Pastore, J. J., MacKintosh, F. C., Lubensky, T. C., and Janmey, P. A. (2005). Nonlinear Elasticity in Biological Gels. Nature 435 (7039), 191-194. doi:10.1038/nature03521

Stricker, J., Falzone, T., and Gardel, M. L. (2010). Mechanics of the F-Actin Cytoskeleton. J. Biomech. 43 (1), 9-14. doi:10.1016/j.jbiomech.2009.09.003

Stupnikov, M. R., Yang, Y., Mori, M., Lu, J., and Cardoso, W. V. (2019). Jagged and Delta-like Ligands Control Distinct Events during Airway Progenitor Cell Differentiation. ELife 8, e50487. doi:10.7554/eLife.50487 
Suki, B., and Bates, J. H. T. (2011). Lung Tissue Mechanics as an Emergent Phenomenon. J. Appl. Physiol. 110 (4), 1111-1118. doi:10.1152/japplphysiol. 01244.2010

Sun, B., Lembong, J., Normand, V., Rogers, M., and Stone, H. A. (2012). Spatialtemporal Dynamics of Collective Chemosensing. Proc. Natl. Acad. Sci. 109 (20), 7753-7758. doi:10.1073/pnas.1121338109

Sun, Z., Guo, S. S., and Fässler, R. (2016). Integrin-mediated Mechanotransduction. J. Cel Biol. 215 (4), 445-456. doi:10.1083/jcb.201609037

Sutherland, R. M., McCredie, J. A., and Inch, W. R. (1971). Growth of Multicell Spheroids in Tissue Culture as a Model of Nodular Carcinomas. J. Natl. Cancer Inst. 46 (1), 113-120.

Swift, J., Ivanovska, I. L., Buxboim, A., Harada, T., Dingal, P. C. D. P., Pinter, J., et al. (2013). Nuclear Lamin-A Scales with Tissue Stiffness and Enhances Matrix-Directed Differentiation. Science 341 (6149), 1240104. doi:10.1126/ science. 1240104

Szabó, A., Melchionda, M., Nastasi, G., Woods, M. L., Campo, S., Perris, R., et al. (2016). In Vivo confinement Promotes Collective Migration of Neural Crest Cells. J. Cel Biol. 213 (5), 543-555. doi:10.1083/jcb.201602083

Takeshita, Y., Obermeier, B., Cotleur, A., Sano, Y., Kanda, T., and Ransohoff, R. M. (2014). An In Vitro Blood-Brain Barrier Model Combining Shear Stress and Endothelial Cell/astrocyte Co-culture. J. Neurosci. Methods 232, 165-172. doi:10.1016/j.jneumeth.2014.05.013

Tambe, D. T., Croutelle, U., Trepat, X., Park, C. Y., Kim, J. H., Millet, E., et al. (2013). Monolayer Stress Microscopy: Limitations, Artifacts, and Accuracy of Recovered Intercellular Stresses. PLoS ONE 8 (2), e55172. doi:10.1371/journal. pone.0055172

Tan, K., Cheng, S., Jugé, L., and Bilston, L. E. (2013). Characterising Soft Tissues under Large Amplitude Oscillatory Shear and Combined Loading. J. Biomech. 46 (6), 1060-1066. doi:10.1016/j.jbiomech.2013.01.028

Tan, Y., Huang, H., Ayers, D. C., and Song, J. (2018). Modulating Viscoelasticity, Stiffness, and Degradation of Synthetic Cellular Niches via Stoichiometric Tuning of Covalent versus Dynamic Noncovalent Cross-Linking. ACS Cent. Sci. 4 (8), 971-981. doi:10.1021/acscentsci.8b00170

Tanner, K., Mori, H., Mroue, R., Bruni-Cardoso, A., and Bissell, M. J. (2012). Coherent Angular Motion in the Establishment of Multicellular Architecture of Glandular Tissues. Proc. Natl. Acad. Sci. 109 (6), 1973-1978. doi:10.1073/pnas.1119578109

Tao, J., and Sun, S. X. (2015). Active Biochemical Regulation of Cell Volume and a Simple Model of Cell Tension Response. Biophysical J. 109 (8), 1541-1550. doi:10.1016/j.bpj.2015.08.025

Tay, S., Hughey, J. J., Lee, T. K., Lipniacki, T., Quake, S. R., and Covert, M. W. (2010). Single-cell NF-Kb Dynamics Reveal Digital Activation and Analogue Information Processing. Nature 466 (7303), 267-271. doi:10.1038/nature09145

Tenje, M., Cantoni, F., Porras Hernández, A. M., Searle, S. S., Johansson, S., Barbe, L., et al. (2020). A Practical Guide to Microfabrication and Patterning of Hydrogels for Biomimetic Cell Culture Scaffolds. Organs-on-a-Chip 2, 100003. doi:10.1016/j.ooc.2020.100003

Tewary, M., Dziedzicka, D., Ostblom, J., Prochazka, L., Shakiba, N., Heydari, T., et al. (2019). High-throughput Micropatterning Platform Reveals Nodal-dependent Bisection of Peri-Gastrulation-Associated versus Preneurulation-Associated Fate Patterning. PLOS Biol. 17 (10), e3000081. doi:10.1371/journal.pbio.3000081

Thakar, K., May, C. K., Rogers, A., and Carroll, C. W. (2017). Opposing Roles for Distinct LINC Complexes in Regulation of the Small GTPase RhoA. Mol. Biol. Cel 28 (1), 182-191. doi:10.1091/mbc.e16-06-0467

Tharmann, R., Claessens, M. M. A. E., and Bausch, A. R. (2007). Viscoelasticity of Isotropically Cross-Linked Actin Networks. Phys. Rev. Lett. 98 (8), 088103. doi:10.1103/PhysRevLett.98.088103

The Physical Sciences - Oncology Centers Network (2013). A Physical Sciences Network Characterization of Non-tumorigenic and Metastatic Cells. Scientific Rep. 3 (1), 1449. doi:10.1038/srep01449

Théry, M. (2010). Micropatterning as a Tool to Decipher Cell Morphogenesis and Functions. J. Cel Sci. 123 (24), 4201-4213. doi:10.1242/jcs.075150

Theveneau, E., Marchant, L., Kuriyama, S., Gull, M., Moepps, B., Parsons, M., et al. (2010). Collective Chemotaxis Requires Contact-dependent Cell Polarity. Developmental Cel 19 (1), 39-53. doi:10.1016/j.devcel.2010.06.012

Theveneau, E., and Mayor, R. (2012). Cadherins in Collective Cell Migration of Mesenchymal Cells. Curr. Opin. Cel Biol. 24 (5), 677-684. doi:10.1016/j.ceb. 2012.08.002
Theveneau, E., and Mayor, R. (2011). Can Mesenchymal Cells Undergo Collective Cell Migration? the Case of the Neural Crest: The Case of the Neural Crest. Cel Adhes. Migration 5 (6), 490-498. doi:10.4161/cam.5.6.18623

Theveneau, E., and Mayor, R. (2013). Collective Cell Migration of Epithelial and Mesenchymal Cells. Cell Mol. Life Sci. 70 (19), 3481-3492. doi:10.1007/s00018012-1251-7

Thiam, H.-R., Vargas, P., Carpi, N., Crespo, C. L., Raab, M., Terriac, E., et al. (2016). Perinuclear Arp2/3-Driven Actin Polymerization Enables Nuclear Deformation to Facilitate Cell Migration through Complex Environments. Nat. Commun. 7 (1), 10997. doi:10.1038/ncomms10997

Thiery, J. P., Acloque, H., Huang, R. Y. J., and Nieto, M. A. (2009). EpithelialMesenchymal Transitions in Development and Disease. Cell 139 (5), 871-890. doi:10.1016/j.cell.2009.11.007

Thoma, C. R., Zimmermann, M., Agarkova, I., Kelm, J. M., and Krek, W. (2014). 3D Cell Culture Systems Modeling Tumor Growth Determinants in Cancer Target Discovery. Adv. Drug Deliv. Rev. 69 (70), 29-41. doi:10.1016/j.addr.2014. 03.001

Thoumine, O., and Ott, A. (1997). Time Scale Dependent Viscoelastic and Contractile Regimes in Fibroblasts Probed by Microplate Manipulation. J. Cel Sci. 110 (Pt 17), 2109-2116

Tlili, S., Gauquelin, E., Li, B., Cardoso, O., Ladoux, B., Delanoë-Ayari, H., et al. (2018). Collective Cell Migration without Proliferation: Density Determines Cell Velocity and Wave Velocity. R. Soc. Open Sci. 5 (5), 172421. doi:10.1098/ rsos. 172421

Tlili, S., Gay, C., Graner, F., Marcq, P., Molino, F., and Saramito, P. (2015). Colloquium: Mechanical Formalisms for Tissue Dynamics. The Eur. Phys. J. E 38 (5), 33. doi:10.1140/epje/i2015-15033-4

Tomaiuolo, G., Barra, M., Preziosi, V., Cassinese, A., Rotoli, B., and Guido, S. (2011). Microfluidics Analysis of Red Blood Cell Membrane Viscoelasticity. Lab. Chip 11 (3), 449-454. doi:10.1039/C0LC00348D

Torzilli, P. A. (1985). Influence of Cartilage Conformation on its Equilibrium Water Partition. J. Orthopaedic Res. 3 (4), 473-483. doi:10.1002/jor.1100030410

Totaro, A., Castellan, M., Battilana, G., Zanconato, F., Azzolin, L., Giulitti, S., et al. (2017). YAP/TAZ Link Cell Mechanics to Notch Signalling to Control Epidermal Stem Cell Fate. Nat. Commun. 8 (1), 15206. doi:10.1038/ ncomms 15206

Trepat, X., Deng, L., An, S. S., Navajas, D., Tschumperlin, D. J., Gerthoffer, W. T., et al. (2007). Universal Physical Responses to Stretch in the Living Cell. Nature 447 (7144), 592-595. doi:10.1038/nature05824

Trepat, X., and Fredberg, J. J. (2011). Plithotaxis and Emergent Dynamics in Collective Cellular Migration. Trends Cel Biol. 21 (11), 638-646. doi:10.1016/j. tcb.2011.06.006

Trepat, X., Wasserman, M. R., Angelini, T. E., Millet, E., Weitz, D. A., Butler, J. P., et al. (2009). Physical Forces during Collective Cell Migration. Nat. Phys. 5 (6), 426-430. doi:10.1038/nphys1269

Tripathi, S., Chakraborty, P., Levine, H., and Jolly, M. K. (2020). A Mechanism for Epithelial-Mesenchymal Heterogeneity in a Population of Cancer Cells. PLOS Comput. Biol. 16 (2), e1007619. doi:10.1371/journal.pcbi.1007619

Truesdell, C., Noll, W., and Antman, S. S. (2004). The Non-linear Field Theories of Mechanics. 3rd ed. Springer.

Tsai, J. H., Donaher, J. L., Murphy, D. A., Chau, S., and Yang, J. (2012). Spatiotemporal Regulation of Epithelial-Mesenchymal Transition Is Essential for Squamous Cell Carcinoma Metastasis. Cancer Cell 22 (6), 725-736. doi:10. 1016/j.ccr.2012.09.022

Tsai, M. A., Frank, R. S., and Waugh, R. E. (1994). Passive Mechanical Behavior of Human Neutrophils: Effect of Cytochalasin B. Biophysical J. 66 (6), 2166-2172. doi:10.1016/S0006-3495(94)81012-4

Tsukita, S., Furuse, M., and Itoh, M. (2001). Multifunctional Strands in Tight Junctions. Nat. Rev. Mol. Cel Biol. 2 (4), 285-293. doi:10.1038/35067088

Turgay, Y., Eibauer, M., Goldman, A. E., Shimi, T., Khayat, M., Ben-Harush, K., et al. (2017). The Molecular Architecture of Lamins in Somatic Cells. Nature 543 (7644), 261-264. doi:10.1038/nature21382

Tusan, C., Man, Y., Zarkoob, H., Johnson, D., Andriotis, O., Thurner, P., et al. (2017). Collective Cell Behaviour in Mechanosensing of Substrate Thickness [Preprint]. Biophysics. doi:10.1101/228478

Ulrich, T. A., de Juan Pardo, E. M., and Kumar, S. (2009). The Mechanical Rigidity of the Extracellular Matrix Regulates the Structure, Motility, and Proliferation 
of Glioma Cells. Cancer Res. 69 (10), 4167-4174. doi:10.1158/0008-5472.CAN08-4859

Urbano, R. L., Furia, C., Basehore, S., and Clyne, A. M. (2017). Stiff Substrates Increase Inflammation-Induced Endothelial Monolayer Tension and Permeability. Biophysical J. 113 (3), 645-655. doi:10.1016/j.bpj.2017.06.033

Urner, S., Kelly-Goss, M., Peirce, S. M., and Lammert, E. (2018). Mechanotransduction in Blood and Lymphatic Vascular Development and Disease. Adv. Pharmacol. 81, 155-208. doi:10.1016/bs.apha.2017.08.009

Vader, D., Kabla, A., Weitz, D., and Mahadevan, L. (2009). Strain-Induced Alignment in Collagen Gels. PLoS ONE 4 (6), e5902. doi:10.1371/journal. pone. 0005902

Valon, L., Etoc, F., Remorino, A., di Pietro, F., Morin, X., Dahan, M., et al. (2015). Predictive Spatiotemporal Manipulation of Signaling Perturbations Using Optogenetics. Biophysical J. 109 (9), 1785-1797. doi:10.1016/j.bpj.2015.08.042

Valon, L., Marín-Llauradó, A., Wyatt, T., Charras, G., and Trepat, X. (2017). Optogenetic Control of Cellular Forces and Mechanotransduction. Nat. Commun. 8 (1), 14396. doi:10.1038/ncomms14396

Van Aelst, L. (2002). Role of Rho Family GTPases in Epithelial Morphogenesis. Genes Development 16 (9), 1032-1054. doi:10.1101/gad.978802

van Helvert, S., and Friedl, P. (2016). Strain Stiffening of Fibrillar Collagen during Individual and Collective Cell Migration Identified by AFM Nanoindentation. ACS Appl. Mater. Inter. 8 (34), 21946-21955. doi:10. 1021/acsami.6b01755

van Zwieten, R. W., Puttini, S., Lekka, M., Witz, G., Gicquel-Zouida, E., Richard, I., et al. (2014). Assessing Dystrophies and Other Muscle Diseases at the Nanometer Scale by Atomic Force Microscopy. Nanomedicine 9 (4), 393-406. doi:10.2217/nnm.12.215

Vedula, S. R. K., Leong, M. C., Lai, T. L., Hersen, P., Kabla, A. J., Lim, C. T., et al. (2012). Emerging Modes of Collective Cell Migration Induced by Geometrical Constraints. Proc. Natl. Acad. Sci. 109 (32), 12974-12979. doi:10.1073/pnas. 1119313109

Veltman, D. (2014). Actin Dynamics: Cell Migration Takes a New Turn with Arpin. Curr. Biol. 24 (1), R31-R33. doi:10.1016/j.cub.2013.11.022

Verkhovsky, A. B., Svitkina, T. M., and Borisy, G. G. (1999). Self-polarization and Directional Motility of Cytoplasm. Curr. Biol. 9 (1), 11-S1. doi:10.1016/S09609822(99)80042-6

Vicsek, T., and Zafeiris, A. (2012). Collective Motion. Phys. Rep. 517 (3-4), 71-140. doi:10.1016/j.physrep.2012.03.004

Vignaud, T., Galland, R., Tseng, Q., Blanchoin, L., Colombelli, J., and Théry, M. (2012). Reprogramming Cell Shape with Laser Nano-Patterning. J. Cel Sci. jcs, 104901. doi:10.1242/jcs.104901

Vigouroux, C., Henriot, V., and Le Clainche, C. (2020). Talin Dissociates from RIAM and Associates to Vinculin Sequentially in Response to the Actomyosin Force. Nat. Commun. 11 (1), 3116. doi:10.1038/s41467-020-16922-1

Vincent, R., Bazellières, E., Pérez-González, C., Uroz, M., Serra-Picamal, X., and Trepat, X. (2015). Active Tensile Modulus of an Epithelial Monolayer. Phys. Rev. Lett. 115 (24), 248103. doi:10.1103/PhysRevLett.115.248103

Vivante, A., Bronshtein, I., and Garini, Y. (2020). Chromatin Viscoelasticity Measured by Local Dynamic Analysis. Biophysical J. 118 (9), 2258-2267. doi:10.1016/j.bpj.2020.04.002

Wagh, A. A., Roan, E., Chapman, K. E., Desai, L. P., Rendon, D. A., Eckstein, E. C., et al. (2008). Localized Elasticity Measured in Epithelial Cells Migrating at a Wound Edge Using Atomic Force Microscopy. Am. J. Physiology-Lung Cell Mol. Physiol. 295 (1), L54-L60. doi:10.1152/ajplung.00475.2007

Wagner, E., and Glotzer, M. (2016). Local RhoA Activation Induces Cytokinetic Furrows Independent of Spindle Position and Cell Cycle Stage. J. Cel Biol. 213 (6), 641-649. doi:10.1083/jcb.201603025

Wang, B. C., Wang, G. R., Yan, D. H., and Liu, Y. P. (1992). An Experimental Study on Biomechanical Properties of Hepatic Tissue Using a New Measuring Method. Bio-Medical Mater. Eng. 2 (3), 133-138.

Wang, N., Tytell, J. D., and Ingber, D. E. (2009). Mechanotransduction at a Distance: Mechanically Coupling the Extracellular Matrix with the Nucleus. Nat. Rev. Mol. Cel Biol. 10 (1), 75-82. doi:10.1038/nrm2594

Wang, R., Wang, Z., Millet, L., Gillette, M. U., Levine, A. J., and Popescu, G. (2011). Dispersion-relation Phase Spectroscopy of Intracellular Transport. Opt. Express 19 (21), 20571. doi:10.1364/OE.19.020571

Wang, Y., Yan, J., and Goult, B. T. (2019). Force-Dependent Binding Constants. Biochemistry 58 (47), 4696-4709. doi:10.1021/acs.biochem.9b00453
Wang, Y., Yao, M., Baker, K. B., Gough, R. E., Le, S., Goult, B. T., et al. (2021). Force-Dependent Interactions between Talin and Full-Length Vinculin. J. Am. Chem. Soc. 143 (36), 14726-14737. doi:10.1021/jacs.1c06223

Watanabe, N., Kato, T., Fujita, A., Ishizaki, T., and Narumiya, S. (1999). Cooperation between mDial and ROCK in Rho-Induced Actin Reorganization. Nat. Cel Biol. 1 (3), 136-143. doi:10.1038/11056

Waugh, R. E., and Bauserman, R. G. (1995). Physical Measurements of BilayerSkeletal Separation Forces. Ann. Biomed. Eng. 23 (3), 308-321. doi:10.1007/ BF02584431

Wear, M. A., Schafer, D. A., and Cooper, J. A. (2000). Actin Dynamics: Assembly and Disassembly of Actin Networks. Curr. Biol. 10 (24), R891-R895. doi:10. 1016/S0960-9822(00)00845-9

Weaver, V. M., Lelièvre, S., Lakins, J. N., Chrenek, M. A., Jones, J. C. R., Giancotti, F., et al. (2002). B4 Integrin-dependent Formation of Polarized ThreeDimensional Architecture Confers Resistance to Apoptosis in normal and Malignant Mammary Epithelium. Cancer Cell 2 (3), 205-216. doi:10.1016/ S1535-6108(02)00125-3

Wei, S. C., Fattet, L., Tsai, J. H., Guo, Y., Pai, V. H., Majeski, H. E., et al. (2015). Matrix Stiffness Drives Epithelial-Mesenchymal Transition and Tumour Metastasis through a TWIST1-G3bp2 Mechanotransduction Pathway. Nat. Cel Biol. 17 (5), 678-688. doi:10.1038/ncb3157

Weihs, D., Mason, T. G., and Teitell, M. A. (2006). Bio-Microrheology: A Frontier in Microrheology. Biophysical J. 91 (11), 4296-4305. doi:10.1529/biophysj.106.081109

Weiswald, L.-B., Bellet, D., and Dangles-Marie, V. (2015). Spherical Cancer Models in Tumor Biology. Neoplasia 17 (1), 1-15. doi:10.1016/j.neo.2014.12.004

Wells, R. G., and Discher, D. E. (2008). Matrix Elasticity, Cytoskeletal Tension, and TGF-: The Insoluble and Soluble Meet. Sci. Signaling 1 (10), pe13. doi:10.1126/ stke.110pe13

Wen, Q., and Janmey, P. A. (2011). Polymer Physics of the Cytoskeleton. Curr. Opin. Solid State. Mater. Sci. 15 (5), 177-182. doi:10.1016/j.cossms.2011.05.002

Wheelock, M. J., Shintani, Y., Maeda, M., Fukumoto, Y., and Johnson, K. R. (2008). Cadherin Switching. J. Cel Sci. 121 (6), 727-735. doi:10.1242/jcs.000455

Wintner, O., Hirsch-Attas, N., Schlossberg, M., Brofman, F., Friedman, R., Kupervaser, M., et al. (2020). A Unified Linear Viscoelastic Model of the Cell Nucleus Defines the Mechanical Contributions of Lamins and Chromatin. Adv. Sci. 7 (8), 1901222. doi:10.1002/advs.201901222

Wipff, P.-J., Rifkin, D. B., Meister, J.-J., and Hinz, B. (2007). Myofibroblast Contraction Activates Latent TGF-B1 from the Extracellular Matrix. J. Cel Biol. 179 (6), 1311-1323. doi:10.1083/jcb.200704042

Wirtz, D., Konstantopoulos, K., and Searson, P. C. (2011). The Physics of Cancer: The Role of Physical Interactions and Mechanical Forces in Metastasis. Nat. Rev. Cancer 11 (7), 512-522. doi:10.1038/nrc3080

Wirtz, D. (2009). Particle-Tracking Microrheology of Living Cells: Principles and Applications. Annu. Rev. Biophys. 38 (1), 301-326. doi:10.1146/annurev. biophys.050708.133724

Wojcieszyn, J. W., Schlegel, R. A., Wu, E. S., and Jacobson, K. A. (1981). Diffusion of Injected Macromolecules within the Cytoplasm of Living Cells. Proc. Natl. Acad. Sci. 78 (7), 4407-4410. doi:10.1073/pnas.78.7.4407

Wolf, K., te Lindert, M., Krause, M., Alexander, S., te Riet, J., Willis, A. L., et al. (2013). Physical Limits of Cell Migration: Control by ECM Space and Nuclear Deformation and Tuning by Proteolysis and Traction Force. J. Cel Biol. 201 (7), 1069-1084. doi:10.1083/jcb.201210152

Wolf, K., Wu, Y. I., Liu, Y., Geiger, J., Tam, E., Overall, C., et al. (2007). Multi-step Pericellular Proteolysis Controls the Transition from Individual to Collective Cancer Cell Invasion. Nat. Cel Biol. 9 (8), 893-904. doi:10.1038/ncb1616

Wozniak, M. A., Desai, R., Solski, P. A., Der, C. J., and Keely, P. J. (2003). ROCKgenerated Contractility Regulates Breast Epithelial Cell Differentiation in Response to the Physical Properties of a Three-Dimensional Collagen Matrix. J. Cel Biol. 163 (3), 583-595. doi:10.1083/jcb.200305010

Wrighton, P. J., and Kiessling, L. L. (2015). Forces of Change: Mechanics Underlying Formation of Functional 3D Organ Buds. Cell Stem Cell 16 (5), 453-454. doi:10.1016/j.stem.2015.04.018

Wu, J., Lewis, A. H., and Grandl, J. (2017). Touch, Tension, and Transduction - the Function and Regulation of Piezo Ion Channels. Trends Biochem. Sci. 42 (1), 57-71. doi:10.1016/j.tibs.2016.09.004

Wu, P.-H., Giri, A., Sun, S. X., and Wirtz, D. (2014). Three-dimensional Cell Migration Does Not Follow a Random Walk. Proc. Natl. Acad. Sci. 111 (11), 3949-3954. doi:10.1073/pnas.1318967111 
Wu, Y. I., Frey, D., Lungu, O. I., Jaehrig, A., Schlichting, I., Kuhlman, B., et al. (2009). A Genetically Encoded Photoactivatable Rac Controls the Motility of Living Cells. Nature 461 (7260), 104-108. doi:10.1038/nature08241

Wullkopf, L., West, A.-K. V., Leijnse, N., Cox, T. R., Madsen, C. D., Oddershede, L. B., et al. (2018). Cancer Cells' Ability to Mechanically Adjust to Extracellular Matrix Stiffness Correlates with Their Invasive Potential. Mol. Biol. Cel 29 (20), 2378-2385. doi:10.1091/mbc.E18-05-0319

Xu, G., and Shao, J.-Y. (2008). Human Neutrophil Surface Protrusion under a point Load: Location independence and Viscoelasticity. Am. J. Physiology-Cell Physiol. 295 (5), C1434-C1444. doi:10.1152/ajpcell.00136.2008

Xu, Q., Engquist, B., Solaimanian, M., and Yan, K. (2020). A New Nonlinear Viscoelastic Model and Mathematical Solution of Solids for Improving Prediction Accuracy. Scientific Rep. 10 (1), 2202.

Xu, W., Mezencev, R., Kim, B., Wang, L., McDonald, J., and Sulchek, T. (2012). Cell Stiffness Is a Biomarker of the Metastatic Potential of Ovarian Cancer Cells. PLoS ONE 7 (10), e46609. doi:10.1371/journal.pone.0046609

Yang, B., Wolfenson, H., Chung, V. Y., Nakazawa, N., Liu, S., Hu, J., et al. (2020). Stopping Transformed Cancer Cell Growth by Rigidity Sensing. Nat. Mater. 19 (2), 239-250. doi:10.1038/s41563-019-0507-0

Yang, Y., Wang, K., Gu, X., and Leong, K. W. (2017). Biophysical Regulation of Cell Behavior-Cross Talk between Substrate Stiffness and Nanotopography. Engineering 3 (1), 36-54. doi:10.1016/J.ENG.2017.01.014

Yano, H., Mazaki, Y., Kurokawa, K., Hanks, S. K., Matsuda, M., and Sabe, H. (2004). Roles Played by a Subset of Integrin Signaling Molecules in CadherinBased Cell-Cell Adhesion. J. Cel Biol. 166 (2), 283-295. doi:10.1083/jcb. 200312013

Yao, M., Goult, B. T., Chen, H., Cong, P., Sheetz, M. P., and Yan, J. (2015). Mechanical Activation of Vinculin Binding to Talin Locks Talin in an Unfolded Conformation. Scientific Rep. 4 (1), 4610. doi:10.1038/srep04610

Yao, M., Goult, B. T., Klapholz, B., Hu, X., Toseland, C. P., Guo, Y., et al. (2016). The Mechanical Response of Talin. Nat. Commun. 7 (1), 11966. doi:10.1038/ ncomms 11966

Yap, A. S., Duszyc, K., and Viasnoff, V. (2018). Mechanosensing and Mechanotransduction at Cell-Cell Junctions. Cold Spring Harbor Perspect. Biol. 10 (8), a028761. doi:10.1101/cshperspect.a028761

Yilmaz, M., and Christofori, G. (2009). EMT, the Cytoskeleton, and Cancer Cell Invasion. Cancer Metastasis Rev. 28 (1-2), 15-33. doi:10.1007/s10555-008-9169-0

Yonemura, S., Wada, Y., Watanabe, T., Nagafuchi, A., and Shibata, M. (2010). aCatenin as a Tension Transducer that Induces Adherens junction Development. Nat. Cel Biol. 12 (6), 533-542. doi:10.1038/ncb2055

Yoon, P. S., Del Piccolo, N., Shirure, V. S., Peng, Y., Kirane, A., Canter, R. J., et al. (2020). Advances in Modeling the Immune Microenvironment of Colorectal Cancer. Front. Immunol. 11, 614300. doi:10.3389/fimmu.2020.614300

Yu, H., Mouw, J. K., and Weaver, V. M. (2011). Forcing Form and Function: Biomechanical Regulation of Tumor Evolution. Trends Cel Biol. 21 (1), 47-56. doi:10.1016/j.tcb.2010.08.015

Yusko, E. C., and Asbury, C. L. (2014). Force Is a Signal that Cells Cannot Ignore. Mol. Biol. Cel 25 (23), 3717-3725. doi:10.1091/mbc.e13-12-0707

Žagar, G., Onck, P. R., and van der Giessen, E. (2015). Two Fundamental Mechanisms Govern the Stiffening of Cross-Linked Networks. Biophysical J. 108 (6), 1470-1479. doi:10.1016/j.bpj.2015.02.015

Zak, A., Merino-Cortés, S. V., Sadoun, A., Mustapha, F., Babataheri, A., Dogniaux, S., et al. (2021). Rapid Viscoelastic Changes Are a Hallmark of Early Leukocyte Activation. Biophysical J. 120 (9), 1692-1704. doi:10.1016/j.bpj.2021.02.042

Zakim, D., Kavecansky, J., and Scarlata, S. (1992). Are Membrane Enzymes Regulated by the Viscosity of the Membrane Environment. Biochemistry 31 (46), 11589-11594. doi:10.1021/bi00161a043

Zangle, T. A., Chun, J., Zhang, J., Reed, J., and Teitell, M. A. (2013). Quantification of Biomass and Cell Motion in Human Pluripotent Stem Cell Colonies. Biophysical J. 105 (3), 593-601. doi:10.1016/j.bpj.2013.06.041

Zangle, T. A., and Teitell, M. A. (2014). Live-cell Mass Profiling: An Emerging Approach in Quantitative Biophysics. Nat. Methods 11 (12), 1221-1228. doi:10. 1038/nmeth.3175

Zervantonakis, I. K., Hughes-Alford, S. K., Charest, J. L., Condeelis, J. S., Gertler, F. B., and Kamm, R. D. (2012). Three-dimensional Microfluidic Model for Tumor Cell Intravasation and Endothelial Barrier Function. Proc. Natl. Acad. Sci. 109 (34), 13515-13520. doi:10.1073/pnas.1210182109
Zhang, J., Tian, X.-J., Zhang, H., Teng, Y., Li, R., Bai, F., et al. (2014). TGF- -induced Epithelial-To-Mesenchymal Transition Proceeds through Stepwise Activation of Multiple Feedback Loops. Sci. Signaling, 7(345), ra91. doi:10.1126/scisignal. 2005304

Zhang, K., Zhu, M., Thomas, E., Hopyan, S., and Sun, Y. (2021). Existing and Potential Applications of Elastography for Measuring the Viscoelasticity of Biological Tissues In Vivo. Front. Phys. 9, 670571. doi:10.3389/fphy.2021. 670571

Zhao, M., Song, B., Pu, J., Wada, T., Reid, B., Tai, G., et al. (2006). Electrical Signals Control Wound Healing through Phosphatidylinositol-3-OH Kinase- $\gamma$ and PTEN. Nature 442 (7101), 457-460. doi:10.1038/nature04925

Zhao, X.-H., Laschinger, C., Arora, P., Szászi, K., Kapus, A., and McCulloch, C. A. (2007). Force Activates Smooth Muscle $\alpha$-actin Promoter Activity through the Rho Signaling Pathway. J. Cel Sci. 120 (10), 1801-1809. doi:10.1242/jcs.001586

Zheng, M., Cierpicki, T., Momotani, K., Artamonov, M. V., Derewenda, U., Bushweller, J. H., et al. (2009). On the Mechanism of Autoinhibition of the RhoA-specific Nucleotide Exchange Factor PDZRhoGEF. BMC Struct. Biol. 9 (1), 36. doi:10.1186/1472-6807-9-36

Zhong, J., Yang, Y., Liao, L., and Zhang, C. (2020). Matrix Stiffness-Regulated Cellular Functions under Different Dimensionalities. Biomater. Sci. 8 (10), 2734-2755. doi:10.1039/C9BM01809C

Zhou, J., Aponte-Santamaría, C., Sturm, S., Bullerjahn, J. T., Bronowska, A., and Gräter, F. (2015). Mechanism of Focal Adhesion Kinase Mechanosensing. PLOS Comput. Biol. 11 (11), e1004593. doi:10.1371/journal.pcbi.1004593

Zhu, C., Chen, Y., and Ju, L. A. (2019). Dynamic Bonds and Their Roles in Mechanosensing. Curr. Opin. Chem. Biol. 53, 88-97. doi:10.1016/j.cbpa.2019. 08.005

Zhu, K., Hum, N. R., Reid, B., Sun, Q., Loots, G. G., and Zhao, M. (2020). Electric Fields at Breast Cancer and Cancer Cell Collective Galvanotaxis. Scientific Rep. 10 (1), 8712. doi:10.1038/s41598-020-65566-0

Zidovska, A., Weitz, D. A., and Mitchison, T. J. (2013). Micron-scale Coherence in Interphase Chromatin Dynamics. Proc. Natl. Acad. Sci. 110 (39), 15555-15560. doi:10.1073/pnas.1220313110

Zimmermann, J., Camley, B. A., Rappel, W.-J., and Levine, H. (2016). Contact Inhibition of Locomotion Determines Cell-Cell and Cell-Substrate Forces in Tissues. Proc. Natl. Acad. Sci. 113 (10), 2660-2665. doi:10.1073/pnas. 1522330113

Zou, J. X., Liu, Y., Pasquale, E. B., and Ruoslahti, E. (2002). Activated Src Oncogene Phosphorylates R-Ras and Suppresses Integrin Activity. J. Biol. Chem. 277 (3), 1824-1827. doi:10.1074/jbc.M103133200

Zuppinger, C. (2019). 3D Cardiac Cell Culture: A Critical Review of Current Technologies and Applications. Front. Cardiovasc. Med. 6, 87. doi:10.3389/ fcvm.2019.00087

Zwerger, M., Ho, C. Y., and Lammerding, J. (2011). Nuclear Mechanics in Disease. Annu. Rev. Biomed. Eng. 13 (1), 397-428. doi:10.1146/annurev-bioeng-071910124736

Zwerger, M., Jaalouk, D. E., Lombardi, M. L., Isermann, P., Mauermann, M., Dialynas, G., et al. (2013). Myopathic Lamin Mutations Impair Nuclear Stability in Cells and Tissue and Disrupt Nucleo-Cytoskeletal Coupling. Hum. Mol. Genet. 22 (12), 2335-2349. doi:10.1093/hmg/ddt079

Conflict of Interest: The author declares that the research was conducted in the absence of any commercial or financial relationships that could be construed as a potential conflict of interest.

Publisher's Note: All claims expressed in this article are solely those of the authors and do not necessarily represent those of their affiliated organizations, or those of the publisher, the editors and the reviewers. Any product that may be evaluated in this article, or claim that may be made by its manufacturer, is not guaranteed or endorsed by the publisher.

Copyright $(2022$ Mierke. This is an open-access article distributed under the terms of the Creative Commons Attribution License (CC BY). The use, distribution or reproduction in other forums is permitted, provided the original author(s) and the copyright owner(s) are credited and that the original publication in this journal is cited, in accordance with accepted academic practice. No use, distribution or reproduction is permitted which does not comply with these terms. 